Aus der Abteilung Klinische Pharmakologie

(Prof. Dr. med. J. Brockmöller)

im Zentrum Pharmakologie und Toxikologie

der Medizinischen Fakultät der Universität Göttingen

\title{
Bedeutung des Cytochrom-P450-2C9- und -3A5- Genpolymorphismus für Pharmakokinetik, Wirkungen und Nebenwirkungen von Delta-9-Tetrahydrocannabinol bei gesunden Probanden und Probandinnen
}

\author{
INAUGURAL-DISSERTATION \\ zur Erlangung des Doktorgrades \\ der Medizinischen Fakultät \\ der Georg-August-Universität zu Göttingen
}

\author{
vorgelegt von \\ Johannes Pfeil \\ aus \\ Rostock
}

Göttingen 2009 
Dekan:

I. Berichterstatter:

II. Berichterstatter/ in:

III. Berichterstatter/ in:
Prof Dr. med. C. Frömmel

Prof. Dr. med. J. Brockmöller

Tag der mündlichen Prüfung: 


\section{Inhaltsverzeichnis}

Inhaltsverzeichnis I

Abkürzungsverzeichnis V

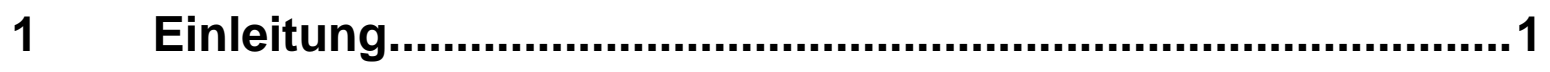

1.1 Pharmakokinetik und Biotransformation ........................................ 1

1.1.1 Bedeutung der Pharmakokinetik für Wirkungen von

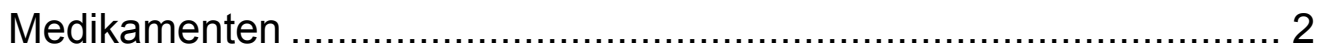

1.1.2 Biotransformation und Elimination von Arzneimitteln ...................... 3

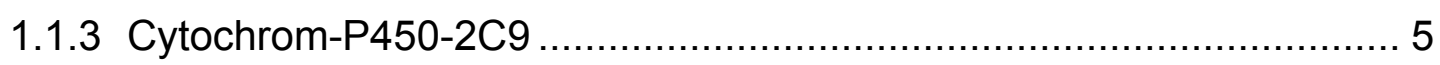

1.2 Pharmakogenetik und Pharmakogenomik ........................................ 7

1.2.1 Allgemeine Einführung zur Pharmakogenetik .............................. 7

1.2.2 Einführung zum CYP2C9-Genpolymorphismus ............................ 8

1.2.3 Einführung zum CYP3A5-Genpolymorphismus ........................... 9

1.2.4 Genvarianten der Influxtransportproteine OATP1B1 .................... 10

1.3 Delta-9-Tetrahydrocannabinol (Dronabinol) .................................. 11

1.3.1 Geschichtliches........................................................... 11

1.3.2 Wirksubstanz Dronabinol ................................................ 12

1.3.3 Pharmakokinetik und Pharmakodynamik von Dronabinol................ 13

1.3.4 Bedeutung von Dronabinol als Medikament .............................. 16

1.3.5 Nebenwirkungen von Dronabinol ......................................... 17

1.3.6 Rechtlicher Status von Dronabinol und Delta-9-THC.................... 18

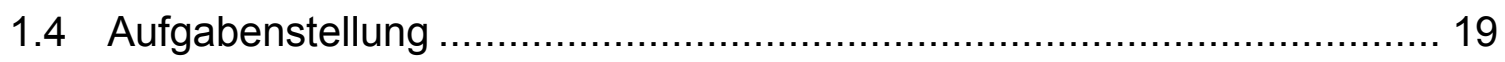

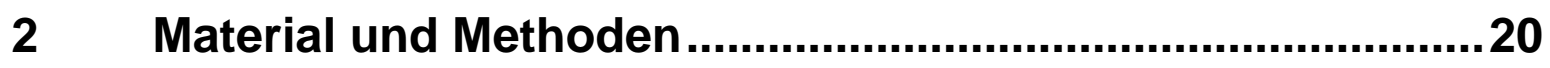

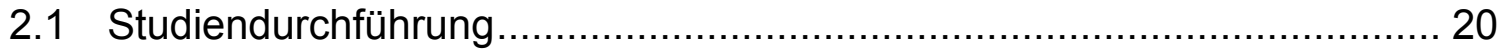

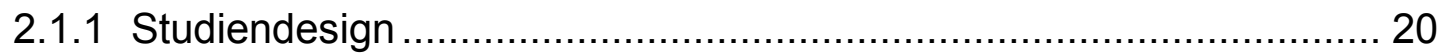

2.1.2 Dokumentation und Datenschutz ....................................... 21

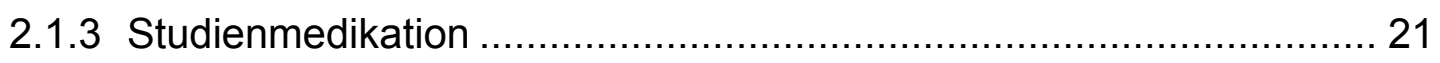

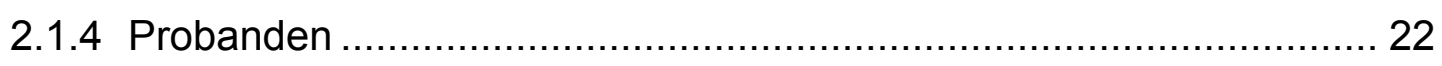

2.1.5 Medizinische Voruntersuchungen ........................................ 23

2.1.6 Diätvorschriften und weitere Verhaltensregeln .......................... 24

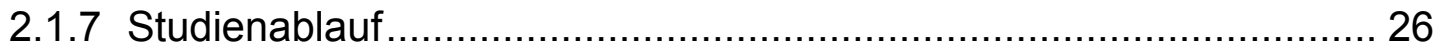

2.1.7.1 Überblick ...................................................................... 26

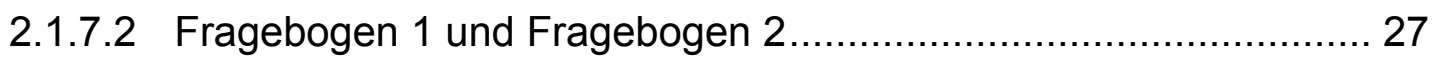




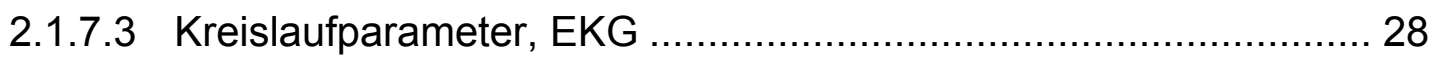

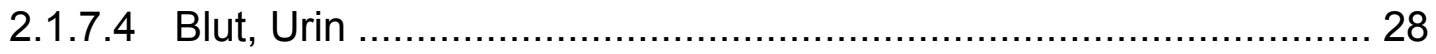

2.1.7.5 Psychometrische Messungen ...................................... 28

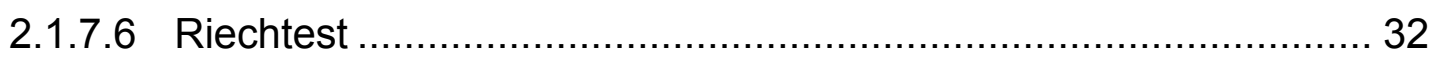

2.1.8 Aufbereitung der gewonnenen Proben ................................... 33

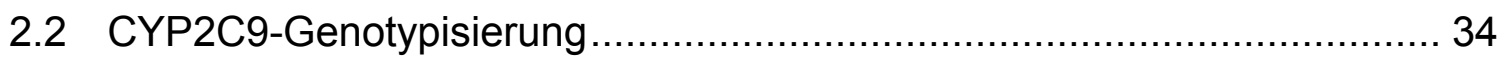

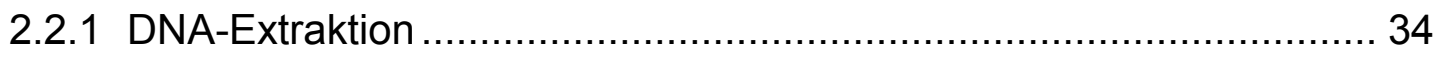

2.2.2 PCR und Fluoreszenzsignalerzeugung .................................. 35

2.2.3 Allelische Diskriminierung ................................................ 36

2.3 Konzentrationsbestimmung des THC und seiner Metabolite im

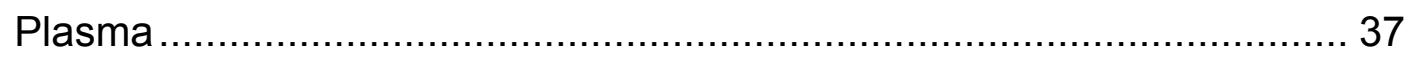

2.4 Konzentrationsbestimmung von $\mathrm{COOH}-\mathrm{THC}$ im Urin .......................... 38

2.5 Pharmakokinetische Auswertungen und Statistik ................................ 39

2.5.1 Pharmakokinetische Begriffe und Parameter............................... 39

2.5.2 Bestimmung pharmakokinetischer Parameter .............................. 41

2.5.3 Statistische Analysen...................................................... 42

2.5.3.1 Fallzahl und Zielgrößen ................................................ 42

2.5.3.2 Exploratorische Datenanalyse .......................................... 43

2.5.3.3 Nichtparametrische Tests.............................................. 43

2.5.3.4 Parametrische statistische Analyse ................................... 44

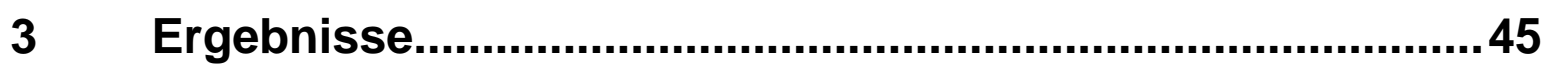

3.1 Untersuchte Probanden und deren molekulargenetische Daten

(Charakteristika der Probandenkohorte) ....................................... 45

3.2 Pharmakokinetische Daten und CYP2C9-Genotyp............................ 47

3.2.1 Konzentrations-Zeit-Verläufe von THC .................................. 47

3.2.2 Pharmakokinetische Parameter von THC ................................. 49

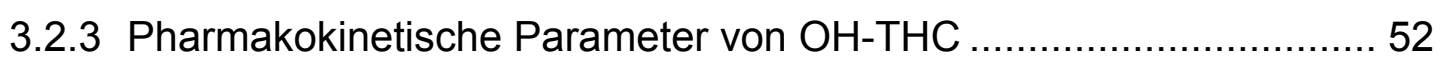

3.2.4 Pharmakokinetische Parameter von $\mathrm{COOH}-\mathrm{THC} \ldots \ldots \ldots \ldots \ldots \ldots \ldots \ldots . . . . . . . . . . . . . . . . .54$

3.3 Analyse der Pharmakokinetik von THC anhand von Kompartiment-

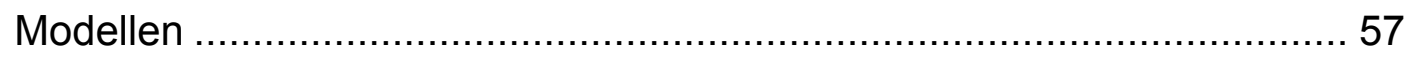

3.4 THC-Pharmakokinetik und CYP3A5-Genpolymorphismus ......................6 60

3.5 Pharmakokinetische Daten in Abhängigkeit vom OATP1B1-

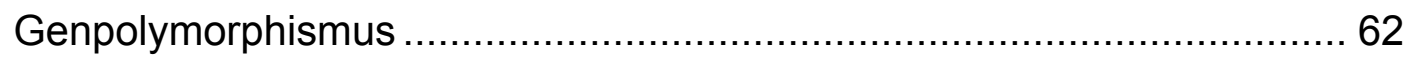

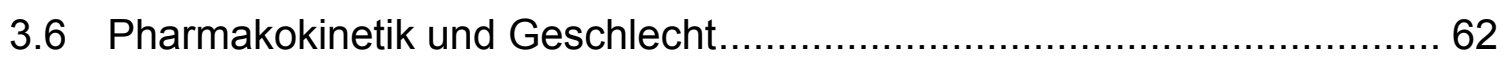




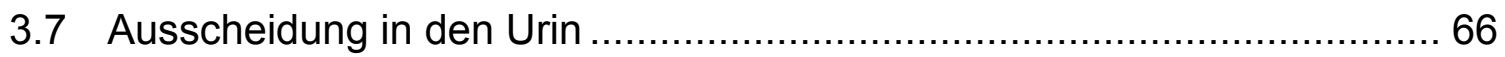

3.7.1 Ausscheidung von THC-COOH in den Urin ............................... 66

3.7.2 Urinausscheidung von $\mathrm{THC}-\mathrm{COOH}$ in Abhängigkeit vom CYP2C9-Genpolymorphismus

3.7.3 Urinausscheidung von $\mathrm{THC}-\mathrm{COOH}$ in Abhängigkeit vom CYP3A5-Genpolymorphismus

3.7.4 Ausscheidung von THC-COOH in Abhängigkeit vom OATP1B1Genpolymorphismus 74

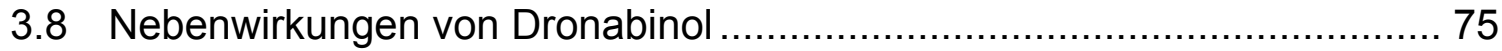

3.8.1 Nebenwirkungen unter THC (Fragebögen 1 und 2) ...................... 75

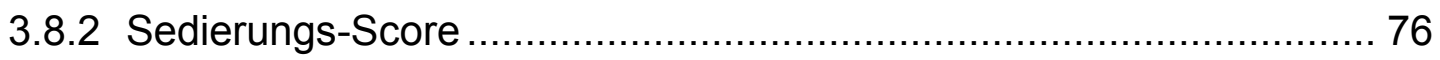

3.8.3 Vegetative Nebenwirkungen anhand von Blutdruck und

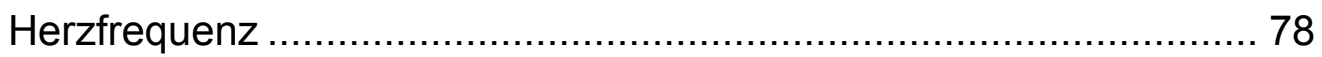

3.9 Pharmakodynamik in Bezug zur Pharmakokinetik und -genetik ............... 80

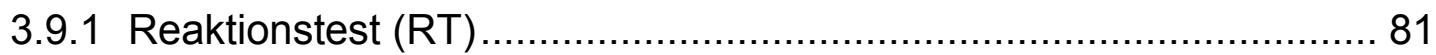

3.9.2 Flimmerfrequenz (FF) und Verschmelzungsfrequenz (VF) ............. 83

3.9.3 Test zur Zeit- und Bewegungsantizipation (ZBA)........................ 84

3.9.4 Fortlaufende visuelle Wiedererkennungsaufgabe (FVW) .................86 86

3.9.5 Erfassung von Stimmungsveränderungen durch den Profile of Mood States (POMS) Test.............................................. 87

3.10 Einfluss von THC und dem CYP2C9-Genotyp auf den Geruchssinn ........ 90

$4 \quad$ Diskussion........................................................................... 92

4.1 Pharmakokinetik im Vergleich zur Literatur.................................... 93

4.1.1 Pharmakokinetik von THC, OH-THC und COOH-THC im Blutplasma .................................................................. 93

4.1.2 Pharmakokinetik von $\mathrm{COOH}-\mathrm{THC}$ im Urin ................................... 96

4.2 Pharmakokinetik und Biotransformation durch CYP2C9 ...................... 97

4.2.1 Einfluss des CYP2C9-Genpolymorphismus auf THC und seine Metaboliten im Plasma

4.2.2 Einfluss des CYP2C9-Genpolymorphismus auf die

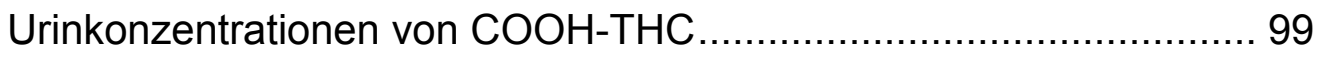

4.3 Bedeutung der Konzentrationsdaten für die forensische Begutachtung . 100

4.4 Beziehungen zwischen Pharmakogenetik, Pharmakokinetik und Pharmakodynamik 
4.4.1 Bedeutung des Endocannabinoidsystems ............................... 102

4.4.2 Neuropsychiatrische Wirkungen von Dronabinol ......................... 103

4.4.3 Vegetative Wirkungen von Dronabinol................................... 106

4.4.4 Einfluss von Dronabinol auf das Riechvermögen ....................... 106

4.5 Ausblick und Verbesserungsmöglichkeiten bei zukünftigen Studien....... 107

$5 \quad$ Zusammenfassung ......................................................... 109

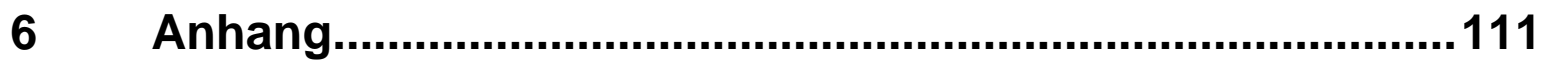

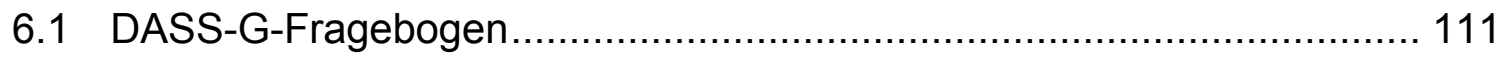

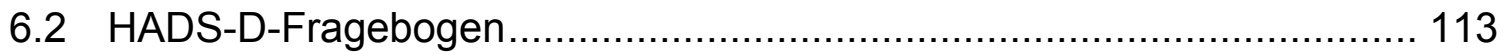

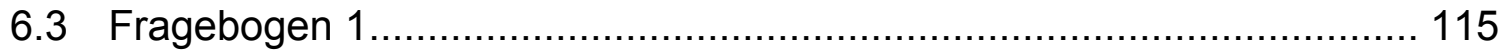

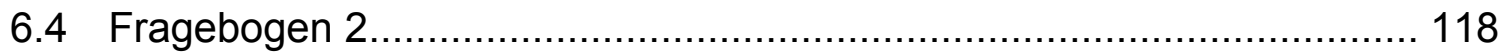

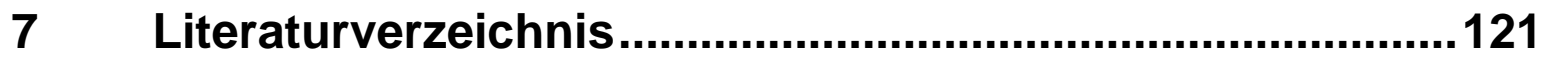




\section{Abkürzungsverzeichnis}

\begin{tabular}{|c|c|}
\hline Ala & Alanin \\
\hline ALT & Alaninaminotransferase \\
\hline Arg & Arginin \\
\hline Asn & Asparagin \\
\hline Asp & Aspartat \\
\hline AST & Aspartataminotransferase \\
\hline AUC & area under the curve \\
\hline BMI & Body Mass Index \\
\hline cDNA & complementary desoxyribonucleic acid \\
\hline $\mathrm{Cl}$ & Clearance \\
\hline $\mathrm{C}_{\max }$ & maximale Konzentration \\
\hline $\mathrm{COOH}$ & Carboxyl-Gruppe einer Carbonsäure \\
\hline CRF & case report form („Dokumentations-Vorlage“) \\
\hline CYP & Cytochrom-P450 \\
\hline CYP2C9 & Cytochrom-P450-2C9 \\
\hline CYP3A5 & Cytochrom-P450-3A5 \\
\hline Cys & Cystein \\
\hline DNA & desoxyribonucleic acid \\
\hline dNTP & Desoxynukleosidtriphosphat \\
\hline EDTA & Ethylendiamintetraacetat \\
\hline EKG & Elektrokardiogramm \\
\hline үGT & Gamma-Glutamyltransferase \\
\hline h & Stunde \\
\hline $\mathrm{Hb}$ & Hämoglobin \\
\hline HF & Herzfrequenz \\
\hline $\mathrm{Hk}$ & Hämatokrit \\
\hline HPLC & high performance liquid chromatography \\
\hline Ile & Isoleucin \\
\hline i.v. & intravenös \\
\hline $\mathrm{K}^{+}$ & Kalium \\
\hline $\mathrm{K}_{\mathrm{m}}$ & Michaelis-Menten-Konstante \\
\hline $\mathrm{kg}$ & Kilogramm \\
\hline
\end{tabular}




\begin{tabular}{|c|c|}
\hline$L$ & Liter \\
\hline Leu & Leucin \\
\hline Lsg. & Lösung \\
\hline $\mathrm{MCH}$ & mean corpuscular haemoglobin \\
\hline $\mathrm{MCHC}$ & mean corpuscular haemoglobin concentration \\
\hline MCV & mean corpuscular volume \\
\hline MDR & Multi Drug Resistance \\
\hline $\mathrm{mg}$ & Milligramm \\
\hline $\mathrm{MgCl}_{2}$ & Magnesiumchlorid \\
\hline $\min$ & Minute \\
\hline $\mathrm{mL}$ & Milliliter \\
\hline$\mu g$ & Mikrogramm \\
\hline$\mu L$ & Mikroliter \\
\hline$\mu \mathrm{m}$ & Mikrometer \\
\hline $\mathrm{ng}$ & Nanogramm \\
\hline $\mathrm{nm}$ & Nanometer \\
\hline $\mathrm{Na}^{+}$ & Natrium \\
\hline $\mathrm{OH}$ & Hydroxid-Gruppe \\
\hline OATP & Organic Anion Transporting Polypeptide \\
\hline PCR & Polymerase Chain Reaction \\
\hline p.o. & per os \\
\hline $\mathrm{RR}$ & Blutdruck nach Riva Rocci \\
\hline s & Sekunde \\
\hline SD & study day \\
\hline SNP & Single Nucleotide Polymorphism \\
\hline SPE & solid phase extraction \\
\hline$t_{1 / 2}$ & Halbwertszeit \\
\hline$t_{\max }$ & Zeitpunkt der maximalen Plasmakonzentration \\
\hline TP & time point \\
\hline$U$ & unit \\
\hline Val & Valin \\
\hline $\mathrm{V}_{\max }$ & maximale Geschwindigkeit (Enzymkinetik) \\
\hline
\end{tabular}




\section{Einleitung}

Beim Konsum illegaler Drogen steht Cannabis seit über 30 Jahren auf Platz eins in Deutschland und Europa. Im Alter von 18 bis 59 Jahren hat in Deutschland etwa jede fünfte Frau und jeder dritte Mann zumindest einmalig Cannabis konsumiert (Tossmann 2006). In Frankreich nehmen in der Altersgruppe von 19 bis 21 Jahren 10.9\% der Frauen und 23.9\% der Männer Cannabis ein (Melchior et al. 2008). Cannabis ist jedoch nicht ausschließlich als illegale Droge zu betrachten, sondern wird seit Jahrtausenden als traditionelles Heilmittel verwendet. Auch heute wird die medizinische Verwendung weiterhin vielfach diskutiert, propagiert und erforscht. Cannabisprodukte können beispielsweise zur Appetitsteigerung sowie zur Verminderung von Chemotherapie-induzierter Übelkeit bei Krebspatienten hilfreich sein (Hall et al. 2005). Des Weiteren sind immunmodulatorische Wirkungen, etwa im Gastrointestinaltrakt, Gegenstand der Forschung (Lu et al. 2006). Dies wird von einigen Autoren als neuer Ansatz in der Therapie von chronisch entzündlichen Darmerkrankungen betrachtet (Izzo und Coutts 2005).

Dronabinol ist der isolierte Hauptwirkstoff der Cannabispflanze und in Deutschland seit 1998 verschreibungsfähig. Bei all diesen Zusammenhängen ist es erstaunlich, dass es bislang erst sehr wenige Untersuchungen zur genetischen Variation in der Pharmakokinetik und den Wirkungen von Cannabinoiden gibt.

\subsection{Pharmakokinetik und Biotransformation}

Arzneimittel unterliegen nach ihrer Aufnahme verschiedenen zwischen Personen variierenden Stoffwechselprozessen, die eine variable Bereitstellung für den Organismus bedingen und auch für Variationen in der Elimination sorgen. Dies wurde in der vorliegenden Arbeit für das Delta-9-Tetrahydrocannabinol, dem wesentlichen Inhaltsstoff von Cannabis (bzw. Marihuana), untersucht.

Die Pharmakokinetik als Teilgebiet der Pharmakologie beschreibt die Gesamtheit aller Prozesse, die auf einen Arzneistoff im Körper einwirken. Unter Biotransformation (Metabolismus) versteht man die chemische Modifikation lipophiler, unpolarer Substanzen in solche mit hydrophilem, polarem Charakter, wodurch ihre Ausscheidung ermöglicht wird. Die Biotransformation ist für die Pharmakokinetik wesentlich. 


\subsubsection{Bedeutung der Pharmakokinetik für Wirkungen von Medikamenten}

Die Gesamtheit pharmakokinetischer Prozesse wird oft in dem Akronym LADME (Liberation, Absorption, Distribution, Metabolismus, Exkretion) zusammengefasst. Nach der Einnahme eines Medikaments erfolgt zunächst die Liberation, d.h. die Freisetzung der Wirksubstanz aus der Arzneiform. Die Liberation beeinflusst dabei Ausmaß und Geschwindigkeit der sich anschließenden Resorption und damit auch die Bioverfügbarkeit des Arzneimittels. Liegt der Wirkstoff bei Applikation bereits in gelöster Form vor, so entfällt dieser Schritt. Als nächstes schließt sich die Aufnahme der Substanz vom Ort der Applikation, bzw. Liberation, in den Blutkreislauf an. Dies wird als Absorption (Resorption) bezeichnet. Neben der Resorption über den Magen-Darm-Trakt können Substanzen auch anderweitig absorbiert werden (z.B. sublingual, nasal, pulmonal oder transdermal). Hat ein Arzneistoff nach vollständiger Resorption die systemische Zirkulation erreicht, erfolgt seine Distribution (Verteilung im Organismus). Meist gelingt diese nicht gleichmäßig im Organismus, sondern es kommt häufig zu Anreicherungen. Hier spielen beispielsweise die Bindung an Plasmaproteine oder auch die Anreicherung im Fettgewebe (bei lipophilen Arzneistoffen) eine Rolle. Die Eigenschaften eines Stoffes, lipophil und unpolar zu sein, führen dazu, dass die Substanz resorbiert werden kann. Andererseits erschweren sie seine Ausscheidung. Es kommt entweder zur renalen Reabsorption oder es erfolgt eine erneute Resorption im Darm nach vorangegangener biliärer Elimination. Eine Ausscheidung wird erst dann möglich, wenn die Substanz hydrophil und polar ist. Die Gesamtheit der biochemischen Prozesse, welche die dazu nötigen Modifikationen der chemischen Struktur vornehmen, bezeichnet man als Metabolismus (Biotransformation). Letztlich erfolgt die Exkretion (Ausscheidung) des Arzneistoffes und seiner Metabolite aus dem Blutkreislauf. Dies geschieht hauptsächlich renal oder biliär über die Galle. Alle LADME-Prozesse beeinflussen den Konzentrations-Zeit-Verlauf und damit auch die Bioverfügbarkeit von Medikamenten im Organismus. Die Bioverfügbarkeit gibt Auskunft über das Ausmaß und die Geschwindigkeit, mit denen ein Arzneistoff in den systemischen Kreislauf gelangt. Bei intravenöser Verabreichung ist sie definitionsgemäß 100\%.

Da Patienten in der Realität individuell variieren, können einheitliche Dosierungen des gleichen Arzneimittels bei verschiedenen Patienten unterschiedliche 
Wirkstoffspiegel und damit auch verschiedene Arzneimittelwirkungen hervorrufen. Bei solchen individuellen Faktoren, die auf die Pharmakokinetik Einfluss nehmen, handelt es sich beispielsweise um die Bioverfügbarkeit des Arzneimittels, aber auch um Körpergröße, Alter und Gewicht sowie die Compliance des Patienten. Des Weiteren können auch andere eingenommene Medikamente und Nahrungsmittel sowie genetisch bedingte Unterschiede die Aktivität arzneimittelmetabolisierender Enzyme beeinflussen (Derendorf et al. 2002). Die Kenntnis der pharmakokinetischen Eigenschaften einer Substanz ist letztendlich die Voraussetzung, um optimale Dosierungsschemata für eine sichere und effektive Arzneimitteltherapie zu schaffen. Dies ist das Ziel der Pharmakokinetik. Zu niedrige Dosierungen können den therapeutischen Erfolg gefährden, zu hohe dagegen toxische Nebenwirkungen bedingen. In der Praxis wird die Bestimmung der Arzneistoffkonzentration im Blut zur Dosisfindung und Therapieüberwachung als Therapeutic Drug Monitoring bezeichnet.

\subsubsection{Biotransformation und Elimination von Arzneimitteln}

Um in den Blutkreislauf zu gelangen, müssen Arzneistoffe in lipophiler, unpolarer Form vorliegen. Diese chemische Konfiguration erschwert allerdings ihre Ausscheidung. Damit solche Substanzen eliminiert werden können, müssen sie in ihrer Struktur zunächst so modifiziert werden, dass sie hydrophil und polar sind. Die dafür nötigen Prozesse werden in ihrer Gesamtheit, wie bereits erwähnt, als Biotransformation bezeichnet. Die entstehenden Arzneistoffmetabolite können entweder eine schwächere, stärkere oder auch eine anderweitige Wirkung im Vergleich zum Ausgangsstoff entfalten. Ebenso können die Metaboliten praktisch unwirksam, aber auch toxisch sein. Ein Arzneimittel wird dann als Prodrug bezeichnet, wenn erst der Metabolit die Wirkform des Ausgangsstoffes darstellt. Ebenso gibt es auch Arzneistoffe, die unverändert ausgeschieden werden. Hinsichtlich der pro Zeiteinheit metabolisierten Arzneistoffmenge spielen die Enzyme in der Leber die größte Rolle.

Bei den Biotransformationsreaktionen unterscheidet man Phase-I-Reaktionen (Funktionalisierung) von Phase-II-Reaktionen (Konjugation). Funktionalisierungsreaktionen sind für den Einbau neuer oder für die Veränderung bereits vorhandener funktioneller Gruppen zuständig, so dass während der Konjugationsreaktionen 
hydrophile Substanzen angekoppelt werden können. Funktionalisierungsreaktionen sind im Wesentlichen Oxidationen, Reduktionen und Hydrolysen. Bezüglich der Anzahl der betroffenen Arzneimittel und der Metabolitenvielfalt nehmen die Oxidationsreaktionen eine herausragende Position ein. Sie werden hauptsächlich durch Cytochrom-P450-Enzyme katalysiert. Zu den wichtigsten Reaktionen der Phase II zählen die Glukuronidierung, die Kopplung mit Glutathion sowie die Sulfatierung und die Acetylierung. Tabelle 1 gibt einen Überblick über bedeutende Reaktionen der Biotransformation und wichtige zugehörige Enzyme.

Tab. 1 Wichtige Reaktionen und Enzyme der Biotransformation

\begin{tabular}{lll}
\hline Reaktionen & Enzyme \\
\hline Oxidation & $\begin{array}{l}\text { Cytochrom-P450-Superfamilie (z. B. CYP2C9, } \\
\text { CYP3A4, CYP3A5) } \\
\text { flavinhaltige Monooxygenasen (FMO) }\end{array}$ \\
Phase I & & $\begin{array}{l}\text { Alkoholdehydrogenase (ADH), } \\
\text { Aldehyddehydrogenase (ALDH) }\end{array}$ \\
& Reduktion & Reduktasen \\
& Hydrolyse & Esterasen \\
\hline \multirow{3}{*}{ Glukuronidierung } & UDP-Glukuronosyltransferasen (UGT) \\
& Glutathionkopplung & Glutathion-S-Transferasen (GST) \\
Phase II & Sulfatierung & Sulfotransferasen \\
& Acetylierung & N-Acetyltransferasen \\
& Konjugation mit Aminosäuren & N-Acyltransferasen \\
\hline
\end{tabular}

Die Konjugation erleichtert schließlich die Ausscheidung über die Nieren mit dem Urin. Das Ausmaß der renalen Elimination lässt sich mit Hilfe der Clearance bestimmen. Eine weitere Möglichkeit der Exkretion ist der biliäre Weg. Dieser wird durch das hohe Molekulargewicht der Konjugate begünstigt. Hierbei gelangt die Substanz über die Galle in den Dünndarm und wird dann mit den Faeces ausgeschieden. Eine erneute Spaltung der Konjugate durch die Enzyme der Darmflora ist möglich. Nach Wiederaufnahme steht der Arzneistoff dem 
systemischen Kreislauf erneut zur Verfügung. Man spricht hierbei vom enterohepatischen Kreislauf (Derendorf et al. 2002).

Viele Faktoren können die Biotransformation von Arzneistoffen beeinflussen. Als Ursachen kommen exogene oder endogene Faktoren in Betracht. Exogene Ursachen sind zum einen die Enzyminduktion, d.h. die Aktivitätszunahme, und zum anderen die Inhibition von Enzymen unter dem Einfluss von bestimmten Substanzen. Dies ist der Fall, wenn mehrere Substrate um dasselbe Enzym konkurrieren. Die Substanz mit der höchsten Affinität zum Enzym kann dieses dann für die anderen konkurrierenden Substrate blockieren. Dadurch kommt es zur verlangsamten Metabolisierung. Endogene Ursachen sind durch genetische Varianten mit reduzierter Enzymfunktion bedingt. Variable Leistungsfähigkeiten des Metabolismus ergeben Unterschiede in Wirkdauer und Wirkstärke vieler Medikamente, da diese von der Geschwindigkeit, mit der sie zu inaktiven Metaboliten umgewandelt werden, abhängig sind.

\subsubsection{Cytochrom-P450-2C9}

Das Cytochrom-P450-2C9 (CYP2C9) gehört zur Superfamilie der Cytochrom-P450Enzyme. Sie nehmen im Arzneimetabolismus des menschlichen Organismus eine herausragende Rolle ein. Die humanen Cytochrom-P450-Enzyme CYP1A2, CYP2C9, CYP2C19, CYP2D6 und CYP3A4 sind die wichtigsten arzneimittelmetabolisierenden Isoformen. Sie katalysieren mehr als $90 \%$ der Oxidationsreaktionen von Arzneistoffen und anderen körperfremden Substanzen in der Phase I der Biotransformation (Williams et al. 2003). Des Weiteren sind sie auch an endogenen Prozessen, wie beispielsweise der Steroidhormonsynthese und dem Fettsäuremetabolismus, beteiligt (Goldstein und de Morais 1994). Bei den Cytochrom-P450-Enzymen handelt es sich um intrazelluläre, membranständige Hämproteine (Williams et al. 2003), die sich im endoplasmatischen Retikulum der Zellen, vor allem in Leber und Darm, finden.

Die Cytochrom-P450-Superfamilie gliedert sich nach der Ähnlichkeit ihrer Aminosäuresequenzen in Familien (CYP + arabische Ziffer, z.B. CYP2) und Subfamilien (CYP + arabische Ziffer + Großbuchstabe, z.B. CYP2C) auf. Eine weitere, der Bezeichnung der Subfamilie folgende arabische Ziffer, bestimmt das Isoenzym (z.B. CYP2C9). Mitglieder einer Familie müssen eine gemeinsame 
Aminosäurefrequenz von über $40 \%$ aufweisen können. Bei den zu einer Subfamilie gehörenden Enzymen müssen es über 55\% sein. Die CYP2C-Subfamilie ist für die Metabolisierung von etwa 20\% aller Arzneimittel verantwortlich (Goldstein 2001) und macht circa $20 \%$ der Gesamtheit der Cytochrom-P450-Enzyme in der Leber aus (Shimada et al. 1994). Sie besteht aus den vier Isoenzymen CYP2C8, CYP2C9, CYP2C18 und CYP2C19 (Goldstein und de Morais 1994).

Das Isoenzym CYP2C9 stellt in der CYP2C-Subfamilie das häufigste Isoenzym der menschlichen Leber dar (Goldstein und de Morais 1994). Es metabolisiert eine Vielzahl von Arzneimitteln, mehrere davon mit geringer therapeutischer Breite. Tabelle 2 zeigt Substrate des CYP2C9-Isoenzyms.

Tab. 2 CYP2C9-Substrate (nach Kirchheiner und Brockmöller 2005)

\begin{tabular}{ll}
\hline Substanzklasse & Vertreter \\
\hline Antidepressiva & Fluoxetin, Amitryptilin \\
Antiepileptika & Phenytoin \\
AT-1-Antagonist & Losartan, Irbesartan \\
Glinide & Nateglinid \\
HMG-CoA-Reduktasehemmer & Fluvastatin \\
Nichtsteroidale Antiphlogistika & Celecoxib, Ibuprofen, Diclofenac, ASS, \\
& Indometacin, Naproxen, Lornoxicam, Meloxicam, \\
Schleifendiuretika & Aceclofenac, Flurbiprofen, Phenylbutazon \\
Sulfonylharnstoffe & Torasemid \\
Vitamin-K-Antagonisten & Glibenclamid, Tolbutamid, Glipizid, Glimepirid \\
Selektive Östrogenrezeptormodulatoren & S-Warfarin, S-Phenprocoumon, S- \\
\hline
\end{tabular}

Bei den meisten dieser Substrate handelt es sich um schwache Säuren mit einer elektronegativen Gruppe, welche die Affinität zum Enzym dadurch bestimmt, dass sie mit einer elektropositiven Gruppe des Enzyms interagiert (Miners und Birkett 1998). Demnach wird die Substratspezifität nicht nur durch die Aminosäuresequenz, sondern auch durch elektrostatische Verhältnisse bestimmt. Beispielhaft lässt sich mittels Rifampicin eine Induktion von CYP2C9 erzielen, inhibitorische Einflüsse gehen von Amiodaron aus (Miners und Birkett 1998; Ohyama et al. 2000). 
Die übrigen Mitglieder der CYP2C-Isoenzyme (CYP2C8, CYP2C18 und CYP2C19) teilen sich zu mehr als $87 \%$ die gleiche Aminosäurestruktur, sind jedoch in ihrer Substratspezifität sehr unterschiedlich (Goldstein und de Morais 1994). Auch sie verstoffwechseln eine Vielzahl von Arzneimitteln.

\subsection{Pharmakogenetik und Pharmakogenomik}

\subsubsection{Allgemeine Einführung zur Pharmakogenetik}

Arzneimittelwirkungen sind heutzutage nicht immer vorhersehbar. Für praktisch alle Medikamente ist bekannt, dass es große Unterschiede in Wirkungen und Nebenwirkungen zwischen den Menschen gibt. Selbst bei Standarddosierungen kann es zum Therapieversagen oder auch zu Überdosierungen mit dem Auftreten toxischer Nebenwirkungen kommen. Im Jahre 1998 konnten Lazarou et al. zeigen, dass die Inzidenz von schweren Nebenwirkungen hospitalisierter US-amerikanischer Patienten im untersuchten Zeitraum von 1966 bis 1996 bei $6,7 \%$ lag. Unvorhersehbare Arzneimittelwirkungen und auch -nebenwirkungen sowie deren Folgen stellen zudem eine enorme finanzielle Belastung für das Gesundheitswesen dar (Meisel et al. 2003).

Die Pharmakogenetik untersucht den Einfluss genetischer Variabilität auf die Wirkung von Arzneimitteln (Evans und McLeod 2003). Sie versucht die Frage zu klären, inwiefern eine genotypadaptierte Dosisanpassung bei Medikamenten sinnvoll erscheint. Dies erfolgt im Hinblick auf Medikamentensicherheit und Therapieerfolg. Auch die Kosten-Nutzen-Relation wird hierbei in Betracht gezogen(Tomalik-Scharte et al. 2008). Genetische Variabilität spielt sowohl in der Pharmakokinetik (Enzyme der Biotransformation, Medikamententransporter), als auch in der Pharmakodynamik (Rezeptoren, Transporter, Enzyme, lonenkanäle, Moleküle der Signaltransduktion, Apoptosegene) eine Rolle. Nahezu alle Enzyme des Fremdstoffmetabolismus weisen genetische Variationen (Polymorphismen) auf, durch die es zu Aktivitätseinschränkungen kommen kann. Dies führt zu pharmakokinetischen Unterschieden, also zu Variationen in der Konzentration von Arzneistoffen und deren Metaboliten im Blut und in den Zielgeweben. Liegen die Polymorphismen in den Genen von Arzneimitteltransportern, werden Aufnahme und Verteilung der entsprechenden Substanzen im Organismus beeinflusst. Liegen die Varianten in den Zielstrukturen 
der Medikamente (z.B. Rezeptoren), so hat dies Auswirkungen auf die Medikamenteneffekte.

\subsubsection{Einführung zum CYP2C9-Genpolymorphismus}

Variationen einzelner Basenpaare in einem DNA-Strang werden als Single Nucleotide Polymorphisms (SNPs) bezeichnet. Sie stellen etwa 90\% aller genetischen Varianten des menschlichen Genoms dar. In den codierenden und regulatorischen Regionen des Enzyms CYP2C9 sind über 50 SNPs beschrieben worden, jedoch stellen neben dem Wildtyp nur zwei weitere Nukleotidvarianten echte Allele dar (Kirchheiner und Brockmöller 2005). Der Wildtyp wird mit CYP2C9*1 bezeichnet und enthält an den entsprechenden Stellen die Aminosäuren Arginin und Isoleucin (Arg144/lle359). Beim Allel CYP2C9*2 findet ein Austausch der Aminosäure Arginin gegen Cystein (Cys144/lle359) statt. Das Vorhandensein dieses Allels wurde erstmals im Jahr 1994 anhand des Arzneimittels S-Warfarin beschrieben (Rettie et al. 1994). Bei der dritten Variante handelt es sich um das Allel CYP2C9*3. Hier ist Isoleucin durch Leucin ersetzt (Arg144/Leu359) (Sullivan-Klose et al. 1996). Des Weiteren existieren noch die Allele ${ }^{*},{ }^{*} 5,{ }^{*} 6$ und ${ }^{*} 11$. Diese scheinen allerdings eine untergeordnete Rolle zu spielen (Kirchheiner und Brockmöller 2005). Die Allele *2 und *3 führen zu einem verminderten Metabolismus. Patienten, die diese Allele tragen, benötigen im Vergleich zu Trägern des Wildtyps beispielsweise geringere Dosierungen des Arzneistoffes Warfarin, um denselben antikoagulatorischen Effekt zu erzielen. CYP2C9*3-Träger wiesen unter der Therapie mit Warfarin ein erhöhtes Blutungsrisiko auf (Aithal et al. 1999). Hinsichtlich der in-vitro-Daten ist die Substrataffinität bei CYP2C9*2 nicht wesentlich beeinflusst. Jedoch ist infolge der geringeren intrinsischen Clearance $\left(\mathrm{V}_{\text {max }} / \mathrm{K}_{\mathrm{m}}\right)$ die Aktivität um etwa $50 \%$ im Gegensatz zu CYP2C9*1 vermindert. Für die meisten Substrate des CYP2C9*3 ist die katalytische Aktivität signifikant reduziert. Ursächlich hierfür sind eine Erhöhung der Michaelis-Menten-Konstanten und eine Verminderung der maximalen Enzymaktivität (Lee et al. 2002). Tabelle 3 stellt die Häufigkeit sowie die zu erwartenden Aktivitätsgrade der wichtigen CYP2C9-Allelkonstellationen dar. 
Tab. 3 CYP2C9-Allelfrequenz und Aktivitätsgrade (nach Kirchheiner und Brockmöller 2005, S. 2)

\begin{tabular}{lllll}
\hline Genotyp & Aktivität & Afrikaner $^{1}$ & Asiaten $^{1}$ & Kaukasier $^{1}$ \\
\hline CYP2C9*1/*1 $^{*}$ & Hoch & 87.0 & 96.5 & 65.3 \\
CYP2C $9{ }^{*} /{ }^{*} 2$ & $\begin{array}{l}\text { Hoch (ggf. leichte } \\
\text { Einschränkung) }\end{array}$ & 8.7 & 0 & 20.4 \\
CYP2C9*2/*2 & Niedrig & 0 & 0 & 0.9 \\
CYP2C9*1/*3 & Niedrig & 4.3 & 3.5 & 11.6 \\
CYP2C9*2/*3 & Niedrig & 0 & 0 & 1.4 \\
CYP2C $9 * 3 / * 3$ & Sehr niedrig & 0 & 0 & 0.4 \\
\hline
\end{tabular}

${ }^{1}$ Häufigkeit in der Population (\%)

\subsubsection{Einführung zum CYP3A5-Genpolymorphismus}

Die menschliche CYP3A-Subfamilie beinhaltet die Isoenzyme CYP3A4, CYP3A5, CYP3A7 und CYP3A43. Diese sind am Metabolismus vieler Arzneistoffe beteiligt. Beispielhaft können hier die Immunmodulatoren Tacrolimus (Loh et al. 2008) und Cyclosporin (Iwasaki 2007) sowie die Kalziumkanalblocker Felodipin (Guo et al. 2007) und Verapamil (Jin et al. 2007) genannt werden. Die cDNA-Sequenz des CYP3A5 wurde erstmals 1989 beschrieben (Schuetz et al. 1989). Von allenCYP3A-Enzymen kommt CYP3A5 die größte Bedeutung beim Arzneistoffmetabolismus zu, denn es repräsentiert etwa $50 \%$ der gesamten hepatischen CYP3A-Aktivität (Kuehl et al. 2001).

Für das Enzym CYP3A5 existieren die Allele *1, *3, *6 und *7. Die Allele *6 und *7 kommen vorwiegend in der afroamerikanischen Population zum Tragen (Kuehl et al. 2001; Hustert et al. 2001). Unter den Kaukasiern ist die häufigste Variante das Allel CYP3A5*3. Homozygote Träger verfügen über keine CYP3A5-Aktivität. Lediglich Personen, die zumindest heterozygot für $C Y P 3 A 5^{\star} 1$ sind, weisen eine CYP3A5-Aktivität auf. Anhand des Arzneimittels Midazolam konnte gezeigt werden, dass Individuen, die für $C Y P 3 A 5^{\star} 3$ homozygot sind (keine CYP3A5-Aktivität) im Vergleich zu denen, die für CYP3A5*1 (aktive Varianten des CYP3A5) hetero- bzw. homozygot sind, über nur etwa die Hälfte der katalytischen Aktivität verfügen (Kuehl et al. 2001).

Nach allen vorliegenden biochemischen Daten wird Tetrahydrocannabinol über CYP2C9, aber auch durch CYP3A-Enzyme metabolisiert (Watanabe et al. 2002). Unter Kaukasiern existieren lediglich die zwei CYP3A-Isoenzyme CYP3A4 und 
CYP3A5 in nennenswerten Mengen. Für die meisten biologischen Substrate und Medikamente sind CYP3A4 und CYP3A5 im korrekten Wortsinne Isoenzyme, d.h. beide katalysieren die gleichen Reaktionen so dass man von einer Gesamt-CYP3A-Aktivität sprechen muss. In der deutschen Bevölkerung sind zwischen 90 und $95 \%$ der Personen homozygote Träger der Genvariante CYP3A5*3/*3 und haben daher keine CYP3A5-Aktivität. Die verbleibenden $5-10 \%$ sind Träger der aktiven Variante CYP $3 A 5^{\star} 1 /{ }^{*} 3$. Damit ist deren Gesamt-CYP3A-Aktivität auch höher als diejenige des Bevölkerungsdurchschnitts.

\subsubsection{Genvarianten der Influxtransportproteine OATP1B1}

OATPs (organic anion transporting polypeptides) sind bedeutende Membrantransportproteine, die den Natrium-unabhängigen Transport einer Vielzahl von amphiphilen organischen Verbindungen vermitteln. Dazu zählen beispielsweise Gallensäuren, Schilddrüsenhormone, organische Anionen, diverse Arzneimittel (Methotrexat, Pravastatin, Rifampicin) und andere Xenobiotika. Es gibt zahlreiche genetische Polymorphismen in den OATPs. Die Superfamilie der OATPs wird daher hinsichtlich der Übereinstimmungsgrade ihrer Aminosäuresequenzen in Familien, Subfamilien und individuelle Gene und Genprodukte unterteilt (Hagenbuch und Meier 2003). Mitglieder der OATP1-Familie weisen eine identische Aminosäuresequenz von $\geq 40 \%$ auf. Bei der Subfamilie (OATP1B) müssen $\geq 60 \%$ identisch sein. Hier gibt es beim Menschen die zwei Vertreter OATP1B1 und OATP1B3.

OATP1B1-Transporter sind leberspezifisch. Sie sind an der basolateralen, sinusoidalen Plasmamembran der Hepatozyten lokalisiert. Mit der Aufnahme ihrer Substrate aus dem portalen Kreislauf in die Leber stellen die OATP1B1-Transportproteine den ersten Schritt der hepatischen Arzneimittelelimination dar. Zu ihren Hauptsubstraten zählen organische Anionen und Gallensalze, un- /konjugiertes Bilirubin, Eicosanoide sowie einige Arzneimittel.

Innerhalb der Subfamilie OATP1B1 existieren individuelle Genprodukte (Proteine). Es wurden diverse Polymorphismen beschrieben, wovon einige mit einer deutlichen Reduktion der intrinsischen Clearance $\left(\mathrm{V}_{\max } / \mathrm{K}_{\mathrm{m}}\right)$ einhergehen können. Dadurch kann die Transportfunktion erheblich beeinträchtigt sein (Hagenbuch und Meier 2004). Transportproteine vom Typ OATP1B1 sind für die Aufnahme des Diuretikums Torasemid in die Leber verantwortlich. Genpolymorphismen dieser Transportproteine 
beeinflussen die Pharmakokinetik von Torasemid. Da es sich hier um ein Substrat des Enzyms CYP2C9 handelt, wird vermutet, dass sie auch für den Transport weiterer CYP2C9-Substrate verantwortlich sein können (Vormfelde et al. 2008). Dazu könnte auch THC zählen. Die in unserer Studie untersuchten Allele waren zum einen das $130_{\text {Aspartat }}$ Allel (Asn130Asp) und zum anderen das $174_{\text {Alanin }}$-Allel (Val174Ala).

\subsection{Delta-9-Tetrahydrocannabinol (Dronabinol)}

\subsubsection{Geschichtliches}

Die Hanfpflanze (Cannabis sativa), wie in Abbildung 1 gezeigt, findet bereits seit tausenden von Jahren Anwendung in der Medizin. Die erste Erwähnung stammt aus einer chinesischen Abhandlung über Heilpflanzen und Therapieverfahren, dem Shen Nung Pen Ts'ao Ching. Sie soll etwa zu Beginn der christlichen Zeitrechnung während der Han-Dynastie verfasst worden sein. Darin wurde Cannabis als Mittel gegen Malaria, Schmerzen und Menstruationsbeschwerden beschrieben. In Europa berichtete der griechische Arzt Dioskurides erstmals $50 \mathrm{v}$. Chr über die medizinische Verwendung von Hanf. Die Benediktinerin Hildegard von Bingen, die während des Mittelalters im heutigen Rheinland-Pfalz lebte, berichtete im Jahr 1150 Folgendes: "Wer Kopfweh und leeres Hirn hat, dem erleichtert der Hanf, wenn er ihn ißt, den Kopfschmerz". Mitte des 19. Jahrhunderts etablierte sich indischer Hanf in der europäischen Schulmedizin. Es wurden erfolgreiche Therapieversuche bei Rheuma, Tollwut, Cholera und Krämpfen beschrieben.

Heutzutage ist Hanf eine weltweit verbreitete Pflanze. Sogenannter Nutzhanf findet als Basis für Farben, Lacke und Waschmittel sowie auch in der Textilindustrie Verwendung. Der Konsum von Cannabispräparaten als Droge ist weltweit gebräuchlich. Im Allgemeinen erfolgt dieser entweder durch das Rauchen oder Verdampfen von Marihuana ("Gras“) oder über die Verwendung von Haschisch. Hierbei handelt es sich um aus der Blüte der Cannabispflanze gewonnenes und zu Blöcken oder Platten gepresstes Harz. Der Gehalt an THC ist hier im Vergleich zu Marihuana bedeutend höher. Es wird meist geraucht oder auch in Speisen und Getränken konsumiert. Auch in der Medizin findet Cannabis gegenwärtig 
Verwendung. In den USA und auch in Deutschland sind entsprechende Präparate auf dem Markt.

Abb. 1 Cannabis sativa (entnommen aus THC Pharm $\mathrm{GmbH}$, o. J.)

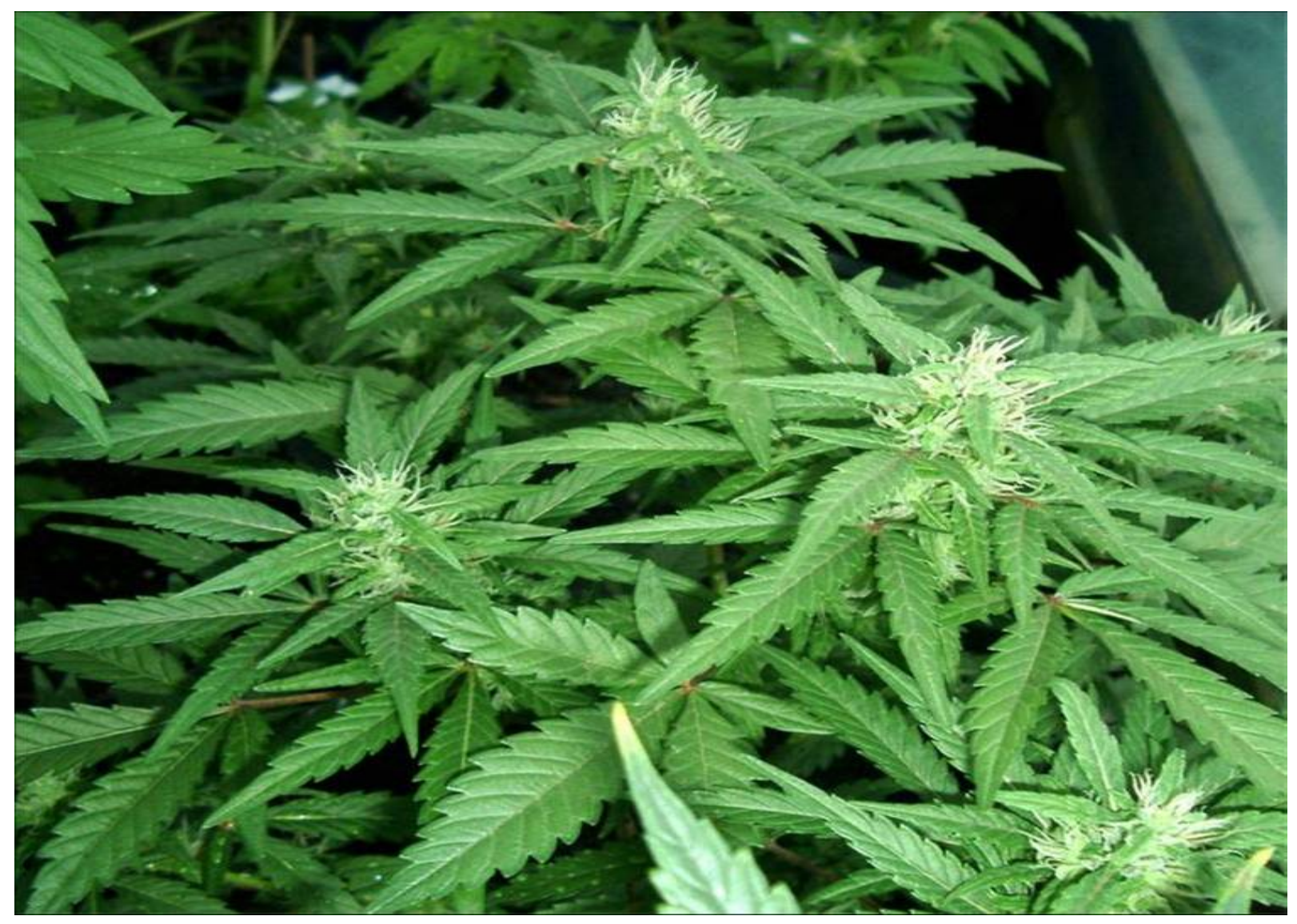

\subsubsection{Wirksubstanz Dronabinol}

Dronabinol (Delta-9-Tetrahydrocannabinol) ist der isolierte Hauptwirkstoff der Cannabispflanze (Cannabis sativa). Als Medikament ist es in Deutschland seit dem 01.02.1998 verschreibungsfähig. Das in unserer Studie verwendete Dronabinol wurde von der Firma THC Pharm (Frankfurt/Main, Deutschland) bezogen. Als Applikationsformen wurden ölige Tropfen (ein Tropfen entspricht $0.83 \mathrm{mg}$ Dronabinol) oder Hartgelatinekapseln (zu 2.5, 5 und $10 \mathrm{mg}$ ) angeboten. Es bestand auch die Möglichkeit zur Inhalation (5\%iges Dronabinol in Ethanol gelöst). In der vorliegenden Studie fanden Dronabinol-Tropfen Verwendung.

In den USA wird Delta-9-THC als Marinol ${ }^{\circledR}$ von der Firma Solvay Pharmaceuticals (Marietta, USA) vertrieben. Hier sind Kapseln zu 2.5, 5 und $10 \mathrm{mg}$ erhältlich. Der 
Hersteller GW Pharmaceuticals (Salisbury, Großbritannien) brachte 2005 das Präparat Sativex® in Kanada auf den Markt. Hierbei handelt es sich um ein Spray, das auf die Mundschleimhaut appliziert wird. Eine Phiole enthält $2.7 \mathrm{mg}$ THC und $2.5 \mathrm{mg}$ Cannabidiol. Die Vorteile der inhalativen und aerosolen Applikation sollen beispielsweise ein geringerer First-Pass-Effekt und die Verminderung von Nebenwirkungen sein. Damit ließe sich insgesamt die Genauigkeit der Dosierung verbessern.

\subsubsection{Pharmakokinetik und Pharmakodynamik von Dronabinol}

Nach oraler Gabe wird Dronabinol nahezu vollständig resorbiert (90 - 95\%). Aufgrund der hohen präsystemisch hepatischen Metabolisierung (First-Pass-Effekt) gelangt jedoch nur eine geringe Dosis in den systemischen Kreislauf. Studienabhängig wird eine orale Bioverfügbarkeit zwischen vier und zwanzig Prozent angegeben (Wall et al. 1983; McGilveray 2005). Dronabinol hat wegen seiner Lipidlöslichkeit ein großes Verteilungsvolumen von ungefähr $10 \mathrm{~L} / \mathrm{kg}$. Die systemische Clearance beträgt bis zu $1 \mathrm{~L} / \mathrm{min}$ (Grotenhermen 2003). Delta-9-THC ist eine hydrophobe schwache Säure (pK 10.6), die vor ihrer Elimination einen umfangreichen Phase-I- und Phase-IIMetabolismus durchläuft (Lemberger et al. 1970). Es bilden sich aktive und inaktive Metabolite. Der aktive Hauptmetabolit des THC ist 11-Hydroxy-Delta-9-THC (OH-THC) (Johnson et al. 1984). $\mathrm{Er}$ erscheint in ungefähr gleicher Plasmakonzentration wie Dronabinol und ist psychoaktiv. Maximalwerte im Plasma zeigen sich etwa zwei bis vier Stunden nach Applikation. Den bedeutendsten inaktiven Metaboliten stellt 11-Nor-9-Carboxy-Delta-9-Tetrahydrocannabinol (COOH-THC) dar. Er entsteht durch Oxidation des OH-THC in der Leber und wird zur Elimination glukuronidiert (Skopp und Pötsch 2002). COOH-THC selbst ist nicht psychoaktiv. Es lässt sich noch Tage bis Wochen nach entsprechendem Konsum nachweisen (Huestis et al. 1995). Abbildung 2 stellt die chemische Struktur von THC und seinen Metaboliten dar. 


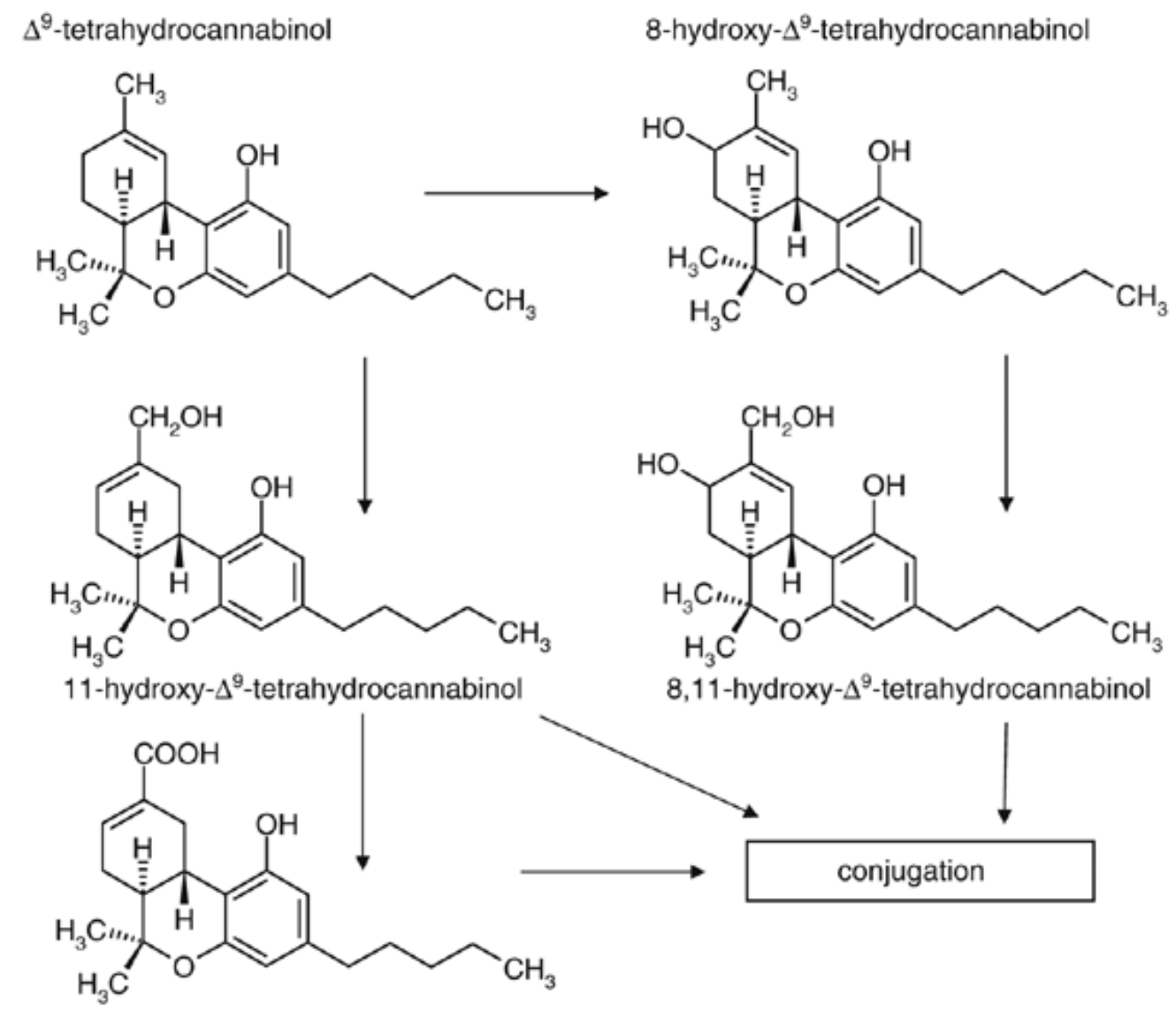

11-nor-9-carboxy- $\Delta^{9}$-tetrahydrocannabinol (CTHC)

Abb. 2 Chemische Struktur von THC und seinen Metaboliten (entnommen aus Glaz-Sandberg et al. 2007, S. 64)

Diversen in-vitro-Studien zufolge wird die Hydroxylierung des THC zu OH-THC durch das polymorphe Enzym CYP2C9 katalysiert (Bornheim et al. 1992; Bland et al. 2005; Nadulski et al. 2005). Es wird vermutet, dass die Subfamilie der CYP3A-Enzyme ebenfalls an der Metabolisierung von Dronabinol beteiligt ist (Bornheim et al. 1992; Bland et al. 2005; Watanabe et al. 2007). Dronabinol und seine Stoffwechselprodukte liegen im Blut zu ungefähr $97 \%$ an Plasmaproteine gebunden vor (Bland et al. 2005). Die Eliminationsphase von Dronabinol wurde in einer Publikation als ZweiKompartiment-Modell (Nadulski et al. 2005), mit einer initialen (Alpha-) Halbwertszeit von vier Stunden und einer terminalen (Beta-) Halbwertszeit von 25 bis 36 Stunden, beschrieben. Dronabinol und seine Biotransformationsprodukte werden in den Faeces und im Urin ausgeschieden. Die größte Bedeutung kommt dabei der biliären Exkretion zu. Von oral appliziertem Dronabinol werden etwa 50\% innerhalb von 72 Stunden mit den Faeces ausgeschieden. Im Urin finden sich 10 bis 15\%, weniger 
als $5 \%$ davon werden unverändert ausgeschieden. Abbildung 3 zeigt die Hauptschritte der Metabolisierung von THC und darauf Einfluss nehmende Faktoren.

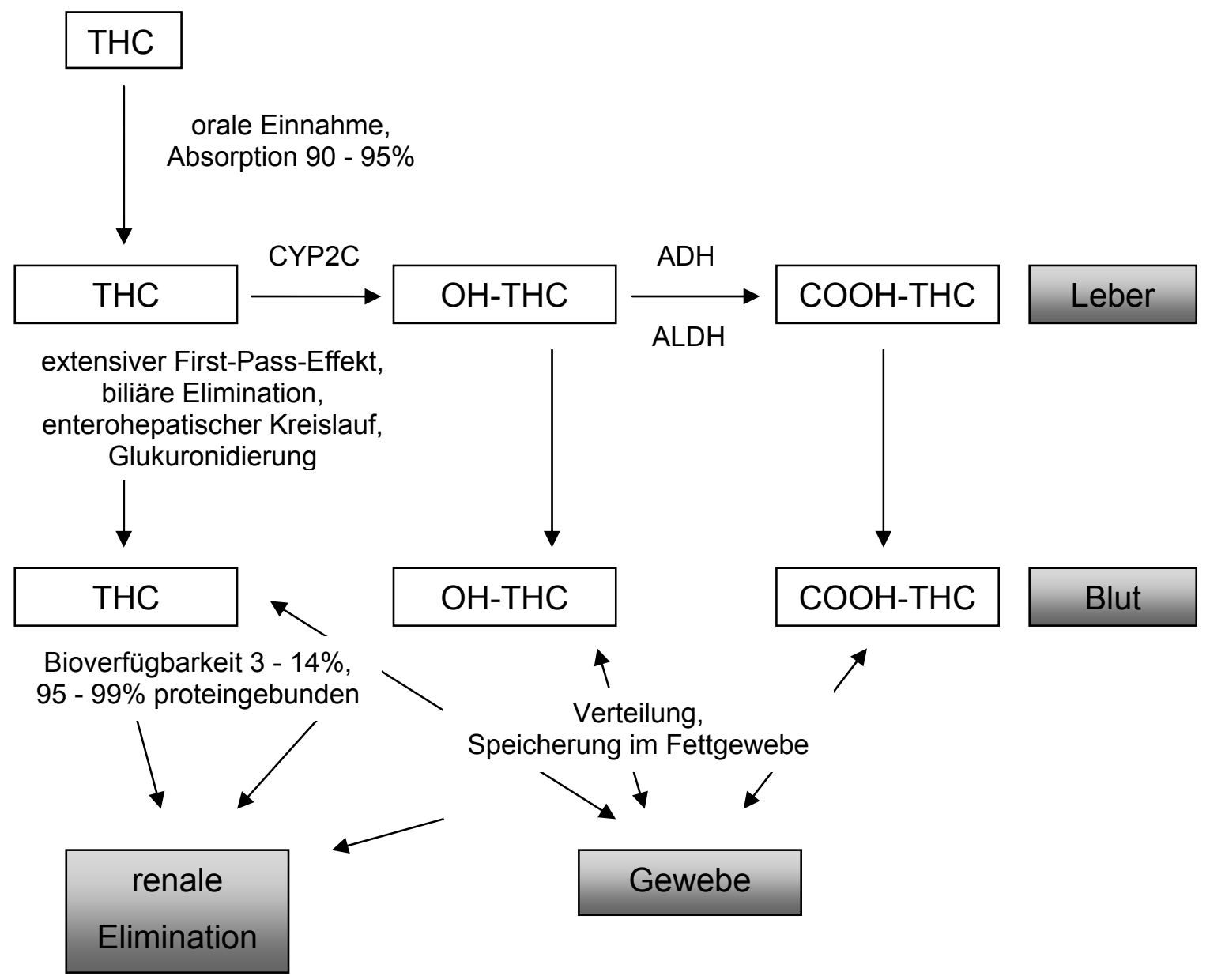

Abb. 3 Metabolisierung von THC und beeinflussende Faktoren bei oraler Applikation (nach Nadulski et al. 2005, S. 808); ADH, Alkoholdehydrogenase; ALDH, Aldehyddehydrogenase

Nach oraler Applikation von Dronabinol kommt es innerhalb von 30 bis 60 Minuten zum Wirkungseintritt. Die maximale Wirkung ist nach 2 bis 4 Stunden erreicht. Psychotrope Effekte treten bis zu 4 bis 6 Stunden nach Applikation auf. Die Appetitstimulation kann bis zu 24 Stunden andauern (THC Pharm GmbH). Wird THC inhaliert, ist nach wenigen Minuten die höchste THC-Konzentration im Blut erreicht. Psychotrope Effekte beginnen nach Sekunden bis Minuten, erreichen ein Maximum nach 15 bis 30 Minuten und klingen nach 1 bis 2 Stunden ab (Grotenhermen 2003). 


\subsubsection{Bedeutung von Dronabinol als Medikament}

Der therapeutische Einsatz von THC ist heute Gegenstand zahlreicher Studien. Patienten mit Multipler Sklerose (MS) können an einer Vielzahl von Symptomen leiden. Dazu zählen beispielsweise Schmerzen, Spastiken, Tremor und Schlafstörungen. Hinsichtlich dieser Symptome können Patienten mit MS von THC profitieren (Deutsch et al. 2008). Insbesondere Schmerzreduktion, erhöhte Mobilität und damit verbesserte Lebensqualität konnten in Studien gut belegt werden. Das Medikament Sativex® ist in Kanada zur Therapie neuropathischer Schmerzen bei Multipler Sklerose wie auch bei opioidrefraktären Tumorschmerzen zugelassen. Bezüglich der Besserung von Spasmen der Muskulatur sind die Studienergebnisse nicht eindeutig (Williamson und Evans 2000; Zajicek et al. 2003; Manzanares et al. 2006). Bei anderen neurologischen Erkrankungen, wie beispielsweise Rückenmarksverletzungen, konnte die Effektivität bei Minderung von Spastiken nach Einnahme von THC nachgewiesen werden (Hagenbach et al. 2007). Ein weiteres Anwendungsgebiet von Dronabinol stellt die Appetitsteigerung, insbesondere bei HIV- und Krebspatienten, dar (Croxford 2003; Ben Amar 2006). Bei der Verminderung von Übelkeit und Erbrechen, vor allem induziert durch Chemotherapeutika, konnten mit der Einnahme von THC gute Ergebnisse erzielt werden (McCabe et al. 1988; Williamson und Evans 2000). Das Medikament Marinol® wurde 1985 in den USA für die Therapie von Übelkeit und Erbrechen sowie zur Appetitsteigerung zugelassen. In Deutschland ist das Indikationsspektrum für Dronabinol darüber hinaus noch auf die Therapie von Spastiken und chronischen Schmerzzuständen erweitert.

Viele weitere Ansatzpunkte in der Therapie mit Cannabinoiden werden heute diskutiert. Dazu haben im Wesentlichen auch die Entdeckung der Cannabinoidrezeptoren sowie die ihrer Liganden, der endogenen Cannabinoide, beigetragen. 1992 konnte Anandamid als erster Ligand des Cannabinoidrezeptors 1 (CNR1, CB1) isoliert werden (Devane et al. 1992). Der CNR1-Rezeptorblocker Rimonabant wurde im Jahr 2006 in Deutschland für die Behandlung der abdominellen Adipositas zugelassen, jedoch Ende 2008 wieder vom Markt genommen. Weitere aktuelle Forschungen werden beispielsweise bezüglich immunmodulierender und antiinflammatorischer Effekte von THC zurzeit durchgeführt. Auch gibt es Ansätze in der Therapie des Asthma bronchiale, des 
Glaukoms, des Tourette-Syndroms, der Epilepsie und der Krebstherapie (Williamson und Evans 2000; Ben Amar 2006; Bifulco et al. 2008).

\subsubsection{Nebenwirkungen von Dronabinol}

Informationen zu unerwünschten Wirkungen stammen aus kontrollierten klinischen Studien mit 474 Patienten, die Marinol ${ }^{8}$ (Dronabinol) erhalten hatten. Studien zur Übelkeit und Erbrechen im Zusammenhang mit einer Krebs-Chemotherapie umfassten 317 Patienten, die Dronabinol, und 157 Patienten, die ein Placebo erhalten hatten. Ein Cannabinoid-Dosis-bezogenes "High" (Neigung zu Lachen, Hochgefühl und erweitertes Bewusstsein) wurde bei Patienten, die Dronabinol erhielten, sowohl bei den antiemetischen $(24 \%$ ) als auch bei den Studien zur Appetitstimulierung mit niedrigeren Dosen $(8 \%)$ beobachtet. Ebenfalls in einem möglichen kausalen Zusammenhang und mit einer Häufigkeit von mehr als $1 \%$ wurden eine Asthenie, Palpitationen, Tachykardie, Gefäßerweiterung/Erröten im Gesicht, Bauchschmerzen sowie Übelkeit und Erbrechen beobachtet. Ferner traten Amnesie, Angst/Nervosität, Ataxie, Verwirrung, Verkennung der eigenen Persönlichkeit, Schwindel, Euphorie, Halluzinationen, paranoide Reaktionen, Schläfrigkeit und abnormes Denken auf. In einer möglicherweise kausalen Beziehung mit einer geringeren Häufigkeit als 1\% traten eine konjunktivale Injektion, niedriger Blutdruck, Durchfall, Stuhlinkontinenz, Myalgien, Depressionen, Albträume, Sprachschwierigkeiten, Tinnitus, Rötung der Haut und Sehschwierigkeiten auf.

Die akute Toxizität von THC ist trotz der geschilderten Nebenwirkungen gering und kann aus den Beobachtungsstudien recht gut eingeschätzt werden. Tödliche Intoxikationen nach millionenfachem Konsum als Rauschdroge oder Anwendungen als Medikament wurden in der Literatur nicht dokumentiert (Grotenhermen 1999). Gemäß Angaben des Herstellers des Handelspräparates Marinol® wird die letale intravenöse Dosis beim Menschen auf $30 \mathrm{mg} / \mathrm{kg}$ geschätzt. Der therapeutische Index wird je nach Quelle mit 1:1.000 bis 1:20.000 angegeben. ZNS-Nebenwirkungen (Sedierung, Dysphorie, Angst, etc.) treten bei oraler Gabe ab etwa 0.2 bis $0.3 \mathrm{mg}$ $\mathrm{THC} / \mathrm{kg}$ Körpergewicht, also bei etwa $20 \mathrm{mg}$ THC für einen Erwachsenen, auf (Cone und Huestis 1993). Die mediane letale Dosis (LD50) von THC lag bei oraler Gabe für Ratten je nach Geschlecht und Stamm bei 800 bis $1900 \mathrm{mg} / \mathrm{kg}$ Körpergewicht. Es wurden keine toxischen Todesfälle bei den maximal 
getesteten THC-Dosen für Hunde (bis $3000 \mathrm{mg} / \mathrm{kg} \mathrm{THC)}$ und Affen(bis $9000 \mathrm{mg} / \mathrm{kg}$ THC) beobachtet (Thompson et al. 1973).

\subsubsection{Rechtlicher Status von Dronabinol und Delta-9-THC}

Zwei Substanzen sind heute in Deutschland über ein Betäubungsmittelrezept verschreibungsfähig. Dabei handelt es sich zum einen um das Medikament Nabilon, ein vollsynthetisches Derivat des Delta-9-THC. Es darf seit 1983 in Deutschland bei HIV- und Krebspatienten eingesetzt werden. Zum anderen ist es der Wirkstoff Dronabinol, welcher seit 1998 verschreibungsfähig ist. Der Gebrauch beider Substanzen wird über Anlage III des Betäubungsmittelgesetzes (BtMG) geregelt. Sowohl Nabilon als auch Dronabinol sind keine nach dem Arzneimittelgesetz (AMG) zugelassenen Substanzen. Sie sind Rezepturarzneimittel und daher nach § 2 AMG von der Zulassungspflicht befreit. Dies wirft das Problem der Kostenübernahme durch die gesetzliche Krankenversicherung (GKV) auf. Daher greifen viele Patienten auf eine Selbstmedikation mit illegalen Cannabisprodukten zurück.

In Deutschland ist bis heute laut BtMG die Verwendung natürlicher Cannabisprodukte, wie zum Beispiel Cannabiskraut (Marihuana), Cannabisharz (Haschisch) und Haschischöl, auch zu medizinischen Zwecken, verboten. In $\S 29$ BtMG heißt es: „Mit Freiheitsstrafe bis zu fünf Jahren oder mit Geldstrafe wird bestraft, wer (...) Betäubungsmittel ohne Erlaubnis nach $\S 3$ Abs. $1 \mathrm{Nr}$. 1 anbaut, herstellt, mit innen Handel treibt, sie, ohne Handel zu treiben, einführt, ausführt, veräußert, abgibt, sonst in den Verkehr bringt, erwirbt oder sich in sonstiger Weise verschafft...". Damit ist jeglicher Besitz von Cannabis in Deutschland strafbar. Eingeschränkt wird dies jedoch durch $\S 31 \mathrm{a}$, in dem es heißt, dass die Staatsanwaltschaft von der Verfolgung absehen kann, „wenn die Schuld des Täters als gering anzusehen wäre, kein öffentliches Interesse an der Strafverfolgung besteht und der Täter die Betäubungsmittel lediglich zum Eigenverbrauch in geringen Mengen anbaut, herstellt, einführt, ausführt, durchführt, erwirbt, sich in sonstiger Weise verschafft oder besitzt". 


\subsection{Aufgabenstellung}

Arzneimittelwirkungen sind heutzutage nicht immer vorhersehbar. Die gleiche Dosis desselben Arzneistoffes kann inter-individuell verschiedene Arzneistoffspiegel hervorrufen. Dies ist auch bei den heutigen Dronabinol-Präparaten der Fall.

In der vorliegenden Studie soll untersucht werden, ob und in welchem Umfang die Pharmakokinetik von Dronabinol durch die genetische Variabilität der für die Biotransformation verantwortlichen Enzyme beeinflusst wird. Die primäre Fragestellung der Studie ist, ob es bedingt durch vordefinierte Genvarianten in den Enzymen CYP2C9 und CYP3A5 zu Unterschieden in den Verläufen der BlutKonzentrations-Zeit-Kurven von Delta-9-THC und seinen Metaboliten kommt. Der Hypothese nach ergibt sich durch die Allele CYP2C9*2 und *3 eine verlangsamte Biotransformation von Dronabinol. Im Hinblick auf das CYP3A5-Enzym soll untersucht werden, ob die 5 bis $10 \%$ der Bevölkerung, welche die aktive Allelkombination $\left({ }^{*} 1 /{ }^{*} 3\right)$ exprimieren, im Gegensatz zu Trägern des Wildtyps $\left({ }^{*} 3 /{ }^{*} 3\right)$, eine schnellere Metabolisierung von Tetrahydrocannabinol zeigen. Exploratorisch soll die Bedeutung genetischer Polymorphismen der Influxtransportproteine vom Typ OATP1B1 untersucht werden, da diese möglicherweise am Transport von THC beteiligt sind. Erkenntnisse zu pharmakodynamischen Aspekten sollen durch psychometrische Messungen ermöglicht werden. Hierzu werden Veränderungen der Stimmung, des Sedierungsgrads, der Wahrnehmung sowie kognitiver Fähigkeiten erfasst. Des Weiteren soll der Einfluss der Studienmedikation auf das Riechvermögen der Probanden untersucht werden.

Eine genauere Kenntnis über Umfang und Ursachen der Variabilität in der Wirkung von THC erscheint sowohl für die medizinische Anwendung, als auch hinsichtlich der missbräuchlichen Verwendung als Rauschdroge bedeutsam. Entsprechende Ergebnisse könnten für die forensische Medizin, insbesondere im Hinblick auf die Interpretation von THC-Plasmakonzentrationen, von Bedeutung sein. 


\section{Material und Methoden}

Aus Gründen der sprachlichen Vereinfachung und zur besseren Lesbarkeit wird im nachfolgenden Text für Probandinnen die männliche Form verwendet. Des Weiteren findet in dieser Arbeit als Dezimaltrenner der Punkt und nicht das Komma Verwendung.

\subsection{Studiendurchführung}

\subsubsection{Studiendesign}

Die Basis der vorliegenden Arbeit bildet eine offene humanpharmakologische Probandenstudie im Parallelgruppendesign. Da es sich bei dem verwendeten Studienmedikament um ein Rezepturarzneimittel handelt, welches nach § 2 AMG von der Zulassungspflicht befreit ist, entspricht die Untersuchung einer humanpharmakologischen Phase-I-Studie an gesunden Probanden. Anhand vorselektionierter CYP2C9- und CYP3A5-Genvariantenträger wurden die AUCs des Wirkstoffs Delta-9-Tetrahydrocannabinol sowie der beiden Metaboliten OH-THC und $\mathrm{COOH}-\mathrm{THC}$ als primäre Zielgrößen untersucht. Sekundäre Zielgrößen waren weitere pharmakokinetische und pharmakodynamische Parameter.

Mittels Anzeigen in der lokalen Presse und Aushängen wurden freiwillige männliche und weibliche Probanden rekrutiert. Zusätzlich wurden vorselektionierte Probanden aus der Probandendatenbank der Klinischen Pharmakologie Göttingen sowie der Bayer-Schering-Pharma AG Berlin kontaktiert. Die Probanden wurden durch die Prüfärzte und den Doktoranden aufgeklärt und anschließend genotypisiert. Bei entsprechender Eignung wurde anschließend ein medizinisches Screening durchlaufen. Fünf Studientage waren zu absolvieren. Die Medikation wurde am zweiten Tag einmalig oral appliziert. Alle Probanden wurden im Vorfeld ihrer Studienteilnahme eingehend mündlich sowie schriftlich durch einen Prüfarzt aufgeklärt und gaben eine schriftliche Einverständniserklärung ab.

Leiter der klinischen Prüfung war Daniel Sehrt aus der Abteilung Klinische Pharmakologie Göttingen. Die Studiendurchführung erfolgte auf der Probandenstation des Instituts für Klinische Pharmakologie im Universitätsklinikum Göttingen. 
Die Untersuchung wurde nach den Richtlinien der Deklaration von Helsinki durchgeführt. Des Weiteren fanden die Grundsätze für die ordnungsgemäße Durchführung der klinischen Prüfung von Arzneimitteln (Bundesanzeiger Nr. 243 vom 30.12.1987 S. 16617 ff), die „Note for Guidance on Good Clinical Practice” (GCP) der ICH, gültig ab 17.1.1997 (Internet source: http://www.eudra.org/emea.html) sowie die Bekanntmachung der Neufassung des Arzneimittelgesetzes (AMG) vom 19. Oktober 1994 (Bundesgesetzblatt, Teil I, 1994:3018-67), geändert durch das 8. Gesetz zur Änderung des AMG vom 7.9.1998 Beachtung. Eine Erlaubnis zum Verkehr mit medizinischem Cannabisextrakt zu wissenschaftlichen Zwecken (BtMG § 3) wurde eingeholt.

\subsubsection{Dokumentation und Datenschutz}

Die Erfassung sämtlicher Befunde und Daten, die bei der Voruntersuchung und während aller Studientage erhoben wurden, erfolgte in für diese Studie entworfenen Dokumentationsbögen (CRF, Case Report Form). Die Zuordnung der CRFs geschah mittels eines fünfstelligen individuellen Codes aus Buchstaben und Ziffern. In derselben Weise wurden Blut- und Urinproben gekennzeichnet. Der Codeschlüssel war nur dem Doktoranden und dem Studienleiter bekannt. Die CRFs wurden während und auch nach Abschluss der Studie in der Abteilung für Klinische Pharmakologie der Universität Göttingen aufbewahrt und sind in einem verschlossenen Schrank in einem für andere Personen nicht zugänglichen Raum aufbewahrt. Eine Weitergabe von Daten an Dritte ist ohne ausdrückliche Zustimmung des Probanden nicht möglich.

\subsubsection{Studienmedikation}

Bei der Studienmedikation handelte es sich um die Substanz Dronabinol (Delta-9-Tetrahydrocannabinol). Die Herstellung der Reinsubstanz erfolgte durch die THC Pharm GmbH, Frankfurt/Main, Deutschland. Die Fertigung der 1\%igen Dronabinol-Lösung (in Sesamöl) wurde durch Mitarbeiter der Apotheke der Medizinischen Fakultät der Universität Ulm durchgeführt. Diese Institution hatte die Herstellungserlaubnis für Prüfpräparate bei Arzneimittelstudien. Die Probanden 
erhielten das Delta-9-THC einmalig oral, in einer Dosierung von $15 \mathrm{mg}$. Dies entsprach $1.5 \mathrm{ml}$ der öligen Dronabinol-Lösung.

\subsubsection{Probanden}

Die Studienteilnehmer waren gesunde Männer und Frauen. Die Rekrutierung weiblicher Probanden war schwieriger, so dass der geplante Anteil von $50 \%$ nicht in jeder Gruppe erreicht werden konnte. Insgesamt nahmen 43 Probanden (27 Männer und 16 Frauen) teil. Die Ein- und Ausschlusskriterien waren im Studienprotokoll festgelegt und werden in Tabelle 4 dargestellt.

Tab. 4 Ein- und Ausschlusskriterien für potentielle Probanden

\begin{tabular}{|c|c|}
\hline Einschlusskriterien & Ausschlusskriterien \\
\hline $\begin{array}{l}18 \text { - } 65 \text { Jahre alte Männer und Frauen bei guter } \\
\text { Gesundheit }\end{array}$ & $\begin{array}{l}\text { Teilnahme an einer anderen klinischen Prüfung } \\
\text { innerhalb der letzten vier Wochen }\end{array}$ \\
\hline Kaukasier europäischer Abstammung & $\begin{array}{l}\text { Blutspende innerhalb der letzen zwei Wochen } \\
\text { oder geplante Blutspende in den nächsten zwei } \\
\text { Wochen }\end{array}$ \\
\hline Gewicht $50-100$ kg & $\begin{array}{l}\text { Bekannte Störungen von Leber- und / oder } \\
\text { Nierenfunktion }\end{array}$ \\
\hline BMI $19-28 \mathrm{~kg} / \mathrm{m}^{2}$ & $\begin{array}{l}\text { Anzeichen für eine aktuelle Infektion von Nieren } \\
\text { und / oder ableitenden Harnwegen oder eine } \\
\text { anderweitig begründete Schrankenstörung, v. a. } \\
\text { der Niere }\end{array}$ \\
\hline $\begin{array}{l}\text { Nichtraucher und Ex-Raucher mit Abstinenz seit } \\
\text { mehr als zwei Monaten }\end{array}$ & $\begin{array}{l}\text { Jedwede chronische Erkrankung oder größere } \\
\text { gastrointestinale Operation }\end{array}$ \\
\hline \multirow[t]{7}{*}{$\begin{array}{l}\text { Willen zur Zusammenarbeit mit dem } \\
\text { Studienpersonal }\end{array}$} & $\begin{array}{l}\text { Laborwerte in klinisch relevantem Maße } \\
\text { außerhalb der üblichen Normwerte, insbesondere } \\
\text { Nieren- oder Leberschädigungsparameter } \\
\text { oberhalb und / oder unterhalb der Norm }\end{array}$ \\
\hline & $\begin{array}{l}\text { Irgendeine regelmäßige Arzneimitteleinnahme } \\
\text { (inklusive hormoneller Kontrazeptiva) }\end{array}$ \\
\hline & $\begin{array}{l}\text { Schwangerschaft oder Stillzeit (Ausschluss erfolgt } \\
\text { mittels Schwangerschaftstest) }\end{array}$ \\
\hline & $\begin{array}{l}\text { Bekannte psychiatrische Erkrankungen } \\
\text { (insbesondere Schizophrenie und Depression) in } \\
\text { der Vorgeschichte }\end{array}$ \\
\hline & $\begin{array}{l}\text { Bekannte Suchterkrankung, insbesondere } \\
\text { Alkohol- oder Opiatbhängigkeit, sowohl aktuell, } \\
\text { als auch jemals in der Anamnese }\end{array}$ \\
\hline & $\begin{array}{l}\text { Jeglicher Konsum von THC in den letzten zwei } \\
\text { Monaten }\end{array}$ \\
\hline & $\begin{array}{l}\text { Jeder Befund, der nach Ansicht des } \\
\text { durchführenden Personals Ablauf oder Ergebnis } \\
\text { der Studie beeinträchtigen könnte }\end{array}$ \\
\hline
\end{tabular}




\subsubsection{Medizinische Voruntersuchungen}

Die persönlichen Daten und eine ausführliche Anamnese wurden mittels verschiedener Fragebögen erfasst. Des Weiteren wurde eine körperliche klinische Untersuchung durchgeführt. Diese beinhaltete auch ein EKG sowie die Messung von Blutdruck, Herzfrequenz und Körpertemperatur. Außerdem erfolgten ein Urinstix- und bei Probandinnen ein Schwangerschaftstest. Ebenso wurde die Haut untersucht, um Hinweise auf intravenösen Drogenkonsum auszuschließen. Weiterer Gegenstand des Screenings waren ein Test auf Atemalkohol (Dräger Alcotest 6510, Drägerwerk AG \& Co. KGaA, Lübeck, Deutschland) und der Ausschluss von THC, (Met-)Amphetaminen, Kokain, Benzodiazepinen und Opiaten im Urin (Mahsan®-Kombi/DOA6-Schnelltest, Mahsan Diagnostika, Reinbek, Deutschland).

Aus Sicherheitsgründen und um sicherzustellen, dass tatsächlich nur gesunde Probanden eingeschlossen wurden, erfolgte die Bestimmung folgender Laborparameter: kleines Blutbild (Hb, Hk, Erythrozyten, Leukozyten, Thrombozyten, $\mathrm{MCV}, \mathrm{MCH}, \mathrm{MCHC}$ ), Natrium, Kalium, Transaminasen (AST, ALT), yGT, GesamtBilirubin, konjugiertes Bilirubin, Albumin und Kreatinin.

Alle Probanden erhielten ein ärztliches Interview, um sicherzustellen, dass keine Personen mit psychischen Erkrankungen in die Studie eingeschlossen wurden. Im Vorfeld der psychiatrischen Anamnese wurden durch die Probanden zwei Fragebögen (FB) ausgefüllt (siehe Anhang). Zum einen der HADS-D (Hospital Anxiety And Depression Scale) Fragebogen. Dies ist die gleichwertige deutsche Adaptation der englischen HADS. 14 Items bilden je eine Angst- und Depressivitäts-Subskala. Der Fragebogen erfasst das Vorhandensein und den Schweregrad milder ängstlicher und depressiver Symptome (Zigmond und Snaith 1983). Zum anderen kam der DASS-G (Depression Anxiety Stress Scales) Fragebogen (verfasst vonDr. Peter F. Lovibond, University of New South Wales, Australia; deutsche Bearbeitung durch Dr. Cecilia A. Essau, Universität Münster) zur Anwendung. Zur Erfassung von Angst, Stress und Depression dienten hierbei 43 Items. Eine Ratingskala von 0 (traf gar nicht auf mich zu) bis 3 (traf sehr stark auf mich zu oder die meiste Zeit) lieferte hier die entsprechenden Ergebnisse (Lovibond und Lovibond 1995). Bei bestehenden oder früheren psychiatrischen Erkrankungen, anderen gesundheitlichen Problemen oder Unzuverlässigkeit des Probanden wurde die betreffende Person nicht in die Studie eingeschlossen. 


\subsubsection{Diätvorschriften und weitere Verhaltensregeln}

Manche Arznei-, aber auch Nahrungs- und Genussmittel sind in der Lage, die Aktivität oxidativer Enzyme zu beeinflussen. Dies betrifft auch die untersuchten Enzyme CYP2C9 und CYP3A5 (Girennavar et al. 2007; Scheen 2007). Um Interaktionen, insbesondere Enzyminduktionen, zu vermeiden, mussten die Probanden während ihrer Studienteilnahme eine „interaktionsarme“ Diät einhalten. Tabelle 5 zeigt die Ernährungsvorgaben.

Tab. 5 Ernährungsvorgaben der Studienteilnehmer

ab einer Woche vor Studienbeginn bis zur letzten Blutentnahme während der Studie ab $48 \mathrm{~h}$ vor, bis $72 \mathrm{~h}$ nach Dronabinoleinnahme
- $\quad$ kein gegrilltes Fleisch oder Gemüse

- $\quad$ keinen Broccoli

- keinerlei Kohlgemüse

- $\quad$ keine Grapefruits oder Grapefruitsaft

- keine Chinin-haltigen Getränke wie Schweppes ${ }^{\circledR}$

- keine hanfhaltigen Produkte

- Schokolade: höchstens $20 \mathrm{~g}$ pro Tag (wichtige Ausnahme: keine hanfhaltige Schokolade)

- Bananen: höchstens 1 Banane pro Tag

- Kakao: höchstens 2 Tassen pro Tag

- Schwarzer oder grüner Tee, Kaffee oder koffeinhaltige Cola-Getränke; insgesamt höchstens 2 Tassen bzw. Gläser pro Tag

- $\quad$ keine Heiltees oder Heilkräuter (wie z.B. Johanniskrauttee), auch nicht gegen prämenstruelles Syndrom (Frauen), Kopfschmerzen oder depressive Verstimmungen

- $\quad$ keine Zubereitungen zur Entschlackung sowie Produkte zum Auf- oder Abbau von Körpermasse

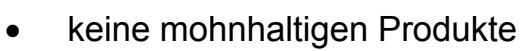

- $\quad$ keine alkoholhaltigen Speisen oder Getränke

- $\quad$ keinen Kaffee, Kakao, keinen schwarzen/grünen Tee, keine Cola, keine Energy-Drinks (Red Bull)

- keine Schokolade

- keine Bananen oder Bananensaft, keine Grapefruit oder Grapefruitsaft 
Sofern die Einnahme von Medikamenten notwendig wurde, erfolgte diese nach Rücksprache mit dem Studienpersonal.

Die Teilnehmer blieben an allen Studientagen von 22 Uhr des Vortages an bis zum Mittagessen nüchtern. Der Konsum kohlensäurehaltiger Getränke wurde ab einer Stunde nach Medikamenteneinnahme gestattet. Ein standardisiertes Mittagessen wurde auf der Probandenstation gestellt. Für weitere Nahrungsmittel und Getränke im Rahmen der interaktionsarmen Diät gab es keine Einschränkungen. 


\subsubsection{Studienablauf}

\subsubsection{1 Überblick}

Die Studie erstreckte sich über fünf aufeinanderfolgende Tage [Baseline, Verum, Studientage 2-4 (SD2-4)]. Einen Überblick zum Studienablauf geben die Tabellen 6 und 7 .

Tab. 6 Ablauf der Studientage

\begin{tabular}{|c|c|c|c|c|c|c|c|c|c|}
\hline TP & Zeit $^{4}$ & Blut & Urin & $\begin{array}{l}\text { Psycho- } \\
\text { metrie }\end{array}$ & $\begin{array}{c}\text { Riech- } \\
\text { test }\end{array}$ & FB1 & FB2 & EKG & $\begin{array}{l}\text { RRI } \\
\text { HF }\end{array}$ \\
\hline TP2 $^{1}$ & $60^{\circ}$ & & & $\Delta$ & & $\Delta$ & $\Delta$ & & $\Delta$ \\
\hline TP4 ${ }^{1}$ & $150^{\circ}$ & & & $\mathbf{\Delta}^{3}$ & & & & & \\
\hline $\mathrm{TP}^{1}$ & $220^{\circ}$ & & & & $\Delta$ & & & & \\
\hline TP8 ${ }^{1}$ & $300^{\circ}$ & & & $\boldsymbol{\Delta}^{3}$ & & & & & \\
\hline TP11 ${ }^{1}$ & $540^{\circ}$ & & & $\Delta$ & & $\Delta$ & & & \\
\hline $\mathrm{TP}^{2}$ & $0^{\circ}$ & $\Delta$ & & & & $\Delta$ & $\Delta$ & & $\Delta$ \\
\hline $\mathrm{TP}^{2}{ }^{2}$ & $30^{\circ}$ & $\Delta$ & & & & & $\Delta$ & & $\Delta$ \\
\hline $\mathrm{TP}^{2}$ & $60^{\circ}$ & $\Delta$ & Fraktion 1 & $\Delta$ & & & $\boldsymbol{\Delta}$ & & $\Delta$ \\
\hline $\mathrm{TP}^{2}$ & $120^{\circ}$ & $\Delta$ & $(0-3 h)$ & & & & $\Delta$ & $\Delta$ & $\Delta$ \\
\hline $\mathrm{TP}^{2}{ }^{2}$ & $150^{\circ}$ & & & $\boldsymbol{\Delta}^{3}$ & & & $\Delta$ & & $\Delta$ \\
\hline TP5 $^{2}$ & $180^{\circ}$ & & & & & & & & $\Delta$ \\
\hline TP6 $^{2}$ & $200^{\circ}$ & $\Delta$ & & & & & $\Delta$ & & $\Delta$ \\
\hline $\mathrm{TP}^{2}$ & $220^{\prime}$ & & Fraktion 2 & & $\Delta$ & & & & \\
\hline TP8 $^{2}$ & $300^{\circ}$ & $\Delta$ & (3-6h) & $\mathbf{\Delta}^{3}$ & & & $\Delta$ & $\Delta$ & $\Delta$ \\
\hline TP9 $^{2}$ & $360^{\circ}$ & & & & & & $\Delta$ & & $\Delta$ \\
\hline TP10 ${ }^{2}$ & $420^{\circ}$ & $\Delta$ & Fraktion 3 & & & & $\Delta$ & & $\Delta$ \\
\hline TP11 ${ }^{2}$ & $540^{\circ}$ & $\Delta$ & (6-9h) & $\Delta$ & & $\Delta$ & $\Delta$ & $\Delta$ & $\Delta$ \\
\hline $\mathrm{TP}_{12}{ }^{2}$ & $720^{\circ}$ & & $\begin{array}{c}\text { Fraktion } 4 \\
(9-12 h)\end{array}$ & & & & & & \\
\hline SD2 & $24 \mathrm{~h}$ & $\Delta$ & $\begin{array}{c}\text { Fraktion } 5 \\
(12-24 h)\end{array}$ & $\mathbf{\Delta}^{3}$ & & $\Delta$ & $\Delta$ & $\Delta$ & $\Delta$ \\
\hline SD3 & $48 \mathrm{~h}$ & $\Delta$ & & $\boldsymbol{\Delta}^{3}$ & & $\Delta$ & $\Delta$ & & $\Delta$ \\
\hline SD4 & $72 \mathrm{~h}$ & $\Delta$ & & $\mathbf{\Delta}^{3}$ & & $\Delta$ & $\Delta$ & & $\Delta$ \\
\hline
\end{tabular}

TP, time point, Zeitpunkt; SD, study day, Studientag; ${ }^{1}$ Messzeitpunkte Baselinetag; ${ }^{2}$ Messzeitpunkte Verumtag (SD1); ${ }^{3}$ Psychometrie mit Gedächtnistest; ${ }^{4}$ Zeit nach Medikamentengabe in Minuten (am Baselinetag erfolgte keine Medikamentengabe, die Zeitpunkte sind denen des Medikamententags äquivalent); FB1, Fragebogen 1; FB2, Fragebogen 2; EKG, Elektrokardiogramm; RR, Blutdruckmessung; HF, Messung der Herzfrequenz 
Tab. 7 Allgemeiner Ablauf der Studie

\begin{tabular}{rlrl}
\hline Baselinetag & Verumtag & SD 4 & SD 5 \\
\hline - & HF, Blutentnahmen & \\
- & Urinfraktionen & \\
- Psychometrische Tests : \\
- & Zeit- und Raumwahrnehmung \\
- & Reaktionsgeschwindigkeit \\
- & Müdigkeit \\
- & Kurzzeitgedächtnis \\
- Fragebögen 1+2 & \\
\hline
\end{tabular}

SD, study day; EKG, Elektrokardiogramm; HF, Messung der Herzfrequenz

\subsubsection{Fragebogen 1 und Fragebogen 2}

Zur Erfassung möglicher vegetativer und psychischer Medikamentennebenwirkungen wurden zwei Fragebögen verwendet (siehe Anhang). Fragebogen 1 musste jeweils zu Beginn und am Ende jedes Studientages ausgefüllt werden. Er umfasste 23 Items in einer anzukreuzenden Ratingskala von null (nein/gar nicht) bis fünf (sehr stark). Fragebogen 1 bezog sich dabei auf die letzten 24 Stunden bis zum Zeitpunkt der Bearbeitung. Er fragte beispielsweise nach dem Auftreten von Schwindel, Übelkeit, Erbrechen, dem Hör- und Sehvermögen, Kopfschmerzen, Konzentrationsfähigkeit und optischen sowie akustischen Halluzinationen. Fragebogen 2 deckte ähnliche Fragenbereiche ab wie Fragebogen 1, bezog sich dabei jedoch im Unterschied zu diesem stets auf die aktuelle Situation der Probanden. Er bestand aus 15 Fragen mit dem identischen Ratingskalenniveau wie Fragebogen 1. Zusätzlich schätzten sowohl die Versuchspersonen, als auch der Untersucher den Grad der Schläfrigkeit der Probanden mit Hilfe der siebenstufigen Stanford Sleepiness Scale (StanfordSedierungs-Skala) ein (Herscovitch und Broughton 1981). Die Skaleneinteilung richtete sich von "1" (sich sehr aktiv, vital, aufmerksam beziehungsweise sehr wach fühlen) bis "7“ (nicht länger gegen den Schlaf ankämpfend; baldiges Einsetzen des Schlafes, beziehungsweise traumartige Gedanken). Des Weiteren wurden mögliche Rötungen der Haut und der Konjunktiven registriert. Fragebogen 2 war am Baselinesowie am Verumtag jeweils 60, 150, 300 und 540 Minuten nach stattgefundener (Verumtag), bzw. am folgenden Verumtag stattfindender (Baselinetag) 
Medikamentengabe zu bearbeiten. Ebenso am Verumtag, vor der Verabreichung des Studienmedikaments, wie auch 30, 120, 360, 420, 720 Minuten sowie 24, 48 und 72 Stunden danach.

\subsubsection{Kreislaufparameter, EKG}

Über alle fünf Studientage erfolgte eine regelmäßige Überwachung des Kreislaufs. Hierzu wurden standardmäßig einmal am Baselinetag, elfmal am Verumtag und an den Studientagen drei bis fünf (SD3 - SD5) jeweils einmal der Blutdruck gemessen und die Herzfrequenz bestimmt. Außerdem wurden am Verumtag in festgelegten Intervallen drei EKGs sowie am dritten Studientag (SD3) ein EKG geschrieben.

\subsubsection{Blut, Urin}

Zwischen den Studientagen eins bis fünf wurde den Probanden insgesamt elfmal venöses Blut entnommen. Hierbei handelte es sich um jeweils zwei 9-mL-EDTA-Röhrchen pro Entnahme. Die entsprechenden Zeitpunkte waren wie folgt festgelegt: vor Medikamentengabe sowie nach 30, 60, 120, 200, 300, 420 und 540 Minuten, als auch 24, 48 und 72 Stunden nach der Applikation des Medikaments.

Die Probanden gaben vor der Medikamenteneinnahme eine Urinprobe ab. Im weiteren Studienverlauf folgten fünf Urinfraktionen, und zwar 0 - 3, 3 - 6, 6 - 9, 9 - 12 und 12 - 24 Stunden nach Medikamentengabe. Zur späteren Analyse wurden aus jeder Fraktion jeweils vier Urinmonovetten zu je $10 \mathrm{~mL}$ entnommen.

\subsubsection{Psychometrische Messungen}

Die zentralnervösen Wirkungen von Dronabinol wurden mit psychometrischen Tests ermittelt. Die folgenden Testverfahren wurden im Vorfeld der Studienteilnahme (maximaler Zeitabstand vier Wochen) durch die Probanden eingeübt, um spätere Lernkurven zu minimieren. Die Testverfahren wurden am Baseline- sowie am Verumtag jeweils 60, 150, 300 und 540 Minuten nach Medikamentengabe, als auch an den Studientagen drei bis fünf (24, 48, 72 Stunden nach Medikamentengabe) in verschiedenen Konstellationen absolviert. Verwendung fand das „Wiener Testsystem“ (Dr. Gernot Schuhfried GmbH, Mödling, Österreich), welches in der 
psychologischen Diagnostik routinemäßig angewendet wird. Für die Durchführung der Tests waren eine spezielle Probandentastatur für alle Testverfahren sowie zur Bestimmung der kritischen Flimmerfusionsfrequenz ein Flimmertubus nötig. Die Auswertungen berücksichtigten Alter und Geschlecht der Probanden mit Hilfe von bereits vorhandenen Normstichproben. Es wurden alle Rohwerte, Prozentränge und T-Werte angegeben. Anschließend konnten die Rohdaten in Microsoft Excel bzw. SPSS für Windows transferiert werden.

Bei der Erfassung des situativen Gefühlszustands fand die deutsche Übersetzung des Profile of Mood States (POMS) Anwendung. Es handelt sich hierbei um eine Selbstbeurteilungsskala, die anhand einer Eigenschaftswörterliste die aktuelle Stimmungslage und die subjektive Befindlichkeit des Probanden abbildet. Die Eigenschaftswörterliste (EWL) ist eine mehrdimensionale Methode zur Beschreibung von Aspekten des Befindens (Janke und Debus 1978). Dargeboten wurden 35 Items. In einer vierfaktoriellen Struktur erfolgte die Skaleneinteilung in Niedergeschlagenheit, Müdigkeit, Tatendrang und Missmut. Einer Instruktion schloss sich die Darbietung der Items auf dem Bildschirm an. Der Proband gab seine Antworten anhand einer siebenstufigen Ratingskala ein. Es war anzugeben, in welchem Maße die dargebotenen Eigenschaften jeweils zutrafen. Die einmalige Korrektur und Überspringen waren gestattet. Es bestand kein Zwang zur Antwort. Zur Auswertung wurden für jede Skala der Rohwert sowie sein prozentualer Anteil am maximal erreichbaren Wert angegeben. Zusätzlich wurde die Bearbeitungszeit erfasst.

Als Verfahren zur Messung der zentralnervösen Aktivierung wurde ein Test zur Bestimmung der Flimmer-/ Verschmelzungsfrequenz (FLIM) angewendet. Hierfür wurde ein Einblicktubus eingesetzt. Im aufsteigenden Verfahren wurde die Frequenz eines Flimmerlichts so lange erhöht, bis der Proband den Eindruck eines ruhigen Lichts (Gleichlicht) wahrgenommen hat. Im absteigenden Verfahren wurde die Frequenz eines höherfrequenten Lichts, welches durch den Probanden als Gleichlicht erkannt wurde, so lange verringert, bis es subjektiv in Flimmerlicht überging. Der Proband hatte entsprechende Wahrnehmungsveränderungen durch Tastendruck zu bestätigen. Die kritische Frequenz wurde gespeichert und die Messungen mehrfach wiederholt. Die Mittelwerte der kritischen Frequenzen sind Schwellenwerte und heißen Verschmelzungsfrequenz (VF) bzw. Flimmerfrequenz (FF). Sie wurden in Hertz (1/s) angegeben. Die VF ergab sich im aufsteigenden 
Verfahren, die FF im absteigenden Verfahren. VF und FF kennzeichnen den Grad des Aktivierungsniveaus. Die Messfehler der VF und FF wurden ebenfalls ausgegeben und dienten als Kontrollvariablen. Sie bestimmten die Genauigkeit, mit der der Proband die Wechsel erfassen konnte. In der Regel liegen die VF-Werte signifikant unter den FF-Werten, was darauf zurückgeführt wird, dass im aufsteigenden Verfahren Nachbilder erzeugt werden, die eine Herabsetzung der VF bewirken (Smith 1971).

Eine in der Verkehrspsychologie wichtige Kenngröße ist das Ausmaß, sich in eine Bewegung einfühlen und die Bewegung von Objekten im Raum richtig einschätzen zu können. Das verwendete Verfahren zur Ermittlung dieser Fähigkeiten war der Test zur Zeit- und Bewegungsantizipation (ZBA). Auf dem Bildschirm erschien eine sich langsam bewegende Kugel. Zu einem unvorhersehbaren Zeitpunkt verschwand diese und es erschienen zwei Linien. Die eine befand sich an der Stelle, an der die Kugel verschwand, die andere Linie war die Ziellinie. Zur Messung der Zeitantizipation sollte der Zeitpunkt des Wiederauftauchens der Kugel an der Ziellinie angegeben werden. Hierfür hatte der Proband in dem Moment eine Taste zu drücken, in dem er der Meinung war, dass die Kugel die Ziellinie erreicht hatte. Um die Bewegungsantizipation zu ermitteln, musste der Proband abschätzen, an welchem Ort die Kugel die Ziellinie erreichen würde. Dazu ließ sich mit Hilfe zweier Tasten ein Pfeil auf dem Bildschirm positionieren. In der Instruktionsphase bekam der Proband eine Rückmeldung bezüglich seiner Leistungen. In der Testphase war dies nicht der Fall. Insgesamt waren 48 Items zu bearbeiten. Zur Auswertung wurde der Zeitfehler als Zeitdifferenz in Sekunden, auf Hundertstelsekunden genau, registriert. Bei der Bewegungsantizipation wurde der Ortsfehler als Abweichung vom korrekten Zielpunkt in Bildpunkten erfasst. Als Hauptvariable dienten dabei die folgenden: mediane Abweichungszeit gesamt (die eine Aussage über die Fähigkeit zur Zeitantizipation erlaubte) und mediane Richtungsabweichung gesamt (mit der die Bewegungsantizipation beurteilt werden konnte). Die Nebenvariablen waren: mediane Abweichungszeit bei linearem Verlauf, mediane Abweichungszeit bei komplexem Verlauf, mediane Abweichungszeit bei sinusförmigem Verlauf, mediane Richtungsabweichung bei linearem Verlauf, mediane Richtungsabweichung bei komplexem Verlauf und mediane Richtungsabweichung bei sinusförmigem Verlauf.

Zur quantifizierten Messung von Gedächtnisleistungen wurde die Fortlaufende Visuelle Wiedererkennungsaufgabe (FVW) verwendet. Bestimmte Items wurden dem 
Probanden der Reihe nach dargeboten. Die Testperson musste durch Drücken einer Taste entscheiden, ob das Item zum ersten oder zum wiederholten Male gezeigt wurde. Jeder Testdurchlauf umfasste 210 Items, bestehend aus 105 verbalen und 105 nonverbalen Items. Der Itempool setzte sich aus Bildern konkreter Gegenstände, Zahlen, Worten, sinnarmen Silben, Buchstaben-, Zahlenkombinationen und schwer zu verbalisierenden Items zusammen. Des Weiteren wurden die Items den Dimensionen konkret/abstrakt, verbal/nonverbal sowie sinnarm/sinnvoll zugeordnet. Die Stimuli erschienen entweder einmal (Distraktoren, „neue Items“) oder zweimal (Attraktoren, Treffer, „alte Items“). Während des Testdurchlaufs waren 100 Treffer und 110 falsch Positive möglich. 10 Items wurden nicht von einem Distraktor zu einem Attraktor überführt. Als Hauptvariablen wurden ausgewertet: die Anzahl der Treffer (Anzahl der Ja-Antworten bei zweimaliger Itemdarbietung), die Anzahl der falsch positiven Treffer (Anzahl der Ja-Antworten bei Distraktoren, d.h. ein bislang nur einmal dargebotenes Item wird als zweimal dargeboten angesehen) sowie die Mittlere Reaktionszeit der Treffer (in Sekunden). Als Nebenvariablen wurden zum einen die Diskriminationsfähigkeit, als Index für die Fähigkeit des Probanden zwischen „alten“ und „neuen“ Items zu unterscheiden erfasst. Es handelte sich um einen Wert zwischen null und eins, wobei eins die maximale Diskriminationsfähigkeit bedeutete. Zum anderen wurde die Antworttendenz als nonparametrisches Maß angegeben. War sie größer als null, lag beim Probanden eine konservative, bei Werten kleiner als null eine liberale Antworttendenz vor. Zusätzlich wurde die Bearbeitungszeit angegeben.

Die Erfassung der Reaktionszeit auf optische und akustische Reize erfolgte durch den Reaktionstest (RT). Bei dieser Testform wurden abwechselnd rotes und gelbes Licht, ein Ton sowie Kombinationen dieser Reize vorgegeben. Die kritische Reizkombination, auf die der Proband reagieren sollte, bestand entweder aus zwei optischen Reizen (gelbes und rotes Licht), oder aus einem optischen und einem akustischen Reiz (gelbes Licht und Ton von $2000 \mathrm{Hertz}$ ). Es waren falsche Reaktionen möglich. In einer Übungsphase wurden 12, in der Testphase 48 Reize vorgegeben. 16 der 48 Reize erforderten eine Reaktion. Als Hauptvariable wurde zum einen die mittlere Reaktionszeit in Millisekunden ausgegeben. Sie entsprach der Zeit zwischen dem Beginn des geforderten Reizes und dem Verlassen der Ruhetaste. Zum anderen war die mittlere motorische Zeit, also die Zeitspanne zwischen dem Verlassen der Ruhetaste und dem Kontakt mit der Drucktaste bei 
geforderten Reizen von Bedeutung. Zu den erfassten Nebenvariablen zählten das Streuungsmaß der Reaktionszeit, welches der Standardabweichung der logarithmisch gewichteten Zeiten entsprach, und das Streuungsmaß der motorischen Zeit, das ebenso als Standardabweichung der logarithmisch gewichteten Zeiten definiert war. Weiterhin wurde die Anzahl der richtigen, falschen, unvollständigen und ausgebliebenen Reaktionen registriert. Als richtige Reaktion galt das Verlassen der Ruhetaste mit anschließender Bestätigung der Reaktionstaste bei geforderten Signalen. Die falsche Reaktion war als Verlassen der Ruhetaste mit anschließender Bestätigung der Reaktionstaste bei nicht geforderten Signalen definiert. Als unvollständige Reaktion galt das Verlassen der Ruhetaste ohne anschließenden Druck auf die Reaktionstaste bei geforderten Signalen. Das Nicht-Verlassen der Ruhetaste bei geforderten Signalen wurde als Nicht-Reaktion (ausgebliebene Reaktion) bezeichnet.

\subsubsection{Riechtest}

Zur Bestimmung der Riechschwelle, bzw. um mögliche Veränderungen in der Diskriminationsfähigkeit zwischen einzelnen Geruchsintensitäten unter Medikamenteneinfluss zu erfassen, wurde ein Riechtest durchgeführt. Hierzu wurden den Probanden je einmal am Baseline- und Verumtag Riechstifte in den Geruchsrichtungen Vanille, Schokolade, Rose, Rauchschinken und Ananas präsentiert. Im Vorfeld jeder Untersuchung wurden Abweichungen vom normalen Geruchssinn, mögliche Erkältungskrankheiten oder eine behinderte Nasenatmung erfragt. Der Riechtest wurde anhand einer aus der Literatur adaptierten Methode durchgeführt (Lötsch et al. 2004). Die Geruchsstoffe wurden in kommerziell verfügbaren Filzstiften präsentiert. Anstelle von Wasserfarben wurden die Tampons mit den entsprechenden Duftlösungen gefüllt, welche mit Propylenglycol als Lösungsmittel verdünnt waren. Die Geruchsstifte wurden in Tripletts präsentiert. Dabei enthielt einer den verdünnten Geruchsstoff, während die zwei weiteren als Leerstifte dienten und lediglich das Lösungsmittel enthielten. Zur Präsentation der Riechstifte wurde die Kappe durch den Versuchsleiter für etwa drei Sekunden entfernt und die Filzspitze des Stiftes wurde ein bis zwei Zentimeter vor die Nasenlöcher des Probanden gehalten. Der Proband wurde zum Riechen aufgefordert. Das Intervall zwischen der Präsentation der individuellen Stifte eines Tripletts betrug etwa drei Sekunden. Die Versuchsperson sollte dabei den Stift 
benennen, der den Geruchsstoff enthielt. Um eine visuelle Erkennung des richtigen Riechstiftes zu vermeiden, trugen die Probanden während des Versuchs eine Augenbinde. Nach dem Abprüfen der 16 Verdünnungsstufen der einen Geruchsrichtung wurde zur nächsten übergegangen. Die Reihenfolge der präsentierten Riechstifte war randomisiert. Der zu erkennende Geruchsstoff war den Versuchspersonen zu jeder Zeit bekannt. Der Proband erfuhr erst am Ende der Messungen, ob seine Angaben richtig oder falsch waren. Die Probanden durften ab 15 Minuten vor Testbeginn lediglich Wasser zu sich nehmen. Vor dem Beginn der Riechschwellenmessung wurden die Probanden mit dem zu erkennenden Geruchsstoff vertraut gemacht.

Bei den Düften Ananas, Schokolade und Schinken handelte es sich jeweils um Parfüme, also Gemische aus verschiedenen Einzelsubstanzen. Bei den Geruchsstoffen Vanille [Stammlösung aus $1 \mathrm{~g}$ Vanillin auf $10 \mathrm{~mL}$ Lösungsmittel (Propylenglycol)] und Rose (2-Phenylethanol) handelte es sich um Reinsubstanzen. Die Düfte wurden in Verdünnungsserien, mit einem Verdünnungsverhältnis von 1:2 und einer Ausgangsverdünnung von $4 \%$ präsentiert.

Die richtigen und falschen Identifizierungen der Proben, die den Geruchsstoff enthielten, wurden erfasst. Die Riechschwelle wurde durch logistische Regression der richtigen und falschen Antworten berechnet.

\subsubsection{Aufbereitung der gewonnenen Proben}

Die zur Genotypisierung bestimmte 9-mL-EDTA-Monovette (Sarstedt Monovette®, Sarstedt AG \& Co, Nümbrecht, Deutschland) wurde mit den Probandendaten und dem Datum beschriftet und anschließend sofort bei $-20^{\circ} \mathrm{C}$ bis zur Analyse aufbewahrt. Die zur Konzentrationsbestimmung abgenommenen Blut- und Urinproben wurden ebenfalls mit den Probandendaten und zusätzlich noch mit den Abnahmezeitpunkten beschriftet. Der Urin wurde auf 10-mL-Röhrchen (Sarstedt Monovette ${ }^{\circledR}$ ) aufgezogen und ohne weitere Behandlung ebenfalls tiefgefroren. Die 9-mL-EDTA-Blutprobe zur Kinetikanalyse wurde nach Abnahme $10 \mathrm{~min}$ bei $3500 \mathrm{U} /$ min zentrifugiert (Heraeus Laborfuge $400 \mathrm{R}$ ). Mit einem Seraplas Plasmafilter wurde das Plasma separiert und in zwei gleich große Portionen auf zwei Greiner-Röhrchen (Greiner Holding AG, Kremsmünster, Österreich) verteilt. Diese wurden nach Beschriftung bei $-20^{\circ} \mathrm{C}$ gelagert. 


\subsection{CYP2C9-Genotypisierung}

Um den Genotyp der Versuchspersonen zu bestimmen, wurden jedem Probanden im Vorfeld der Studie einmalig je zwei 9-mL-Röhrchen EDTA-Blut entnommen. Probe 1 wurde zur Genotypisierung verwendet, Probe 2 verblieb als Kontrolle tiefgefroren bei $-26^{\circ} \mathrm{C}$ auf der Probandenstation. Aus dem EDTA-Vollblut wurde zunächst die DNA extrahiert und anschließend mittels PCR amplifiziert. Danach erfolgte die Diskriminierung der CYP2C9*2- und *3-Allele. Bei dem Enzym CYP3A5 handelte es sich dabei um das Allel CYP3A5*1. Zur Analyse wurde das 7900HT Sequence Detection System (Applied Biosystems, Carlsbad, USA) verwendet.

Die Genotypisierung erfolgte durch Labormitarbeiter der Abteilung für Klinische Pharmakologie der Universität Göttingen.

\subsubsection{DNA-Extraktion}

Die Isolierung der DNA erfolgte als automatisierte Festphasen-Extraktion mittels des Gerätes Bio Robot EZ1 (Qiagen, Germantown, USA). Der Bio Robot EZ1 arbeitet nach dem Prinzip der Trennung der an magnetische Partikel gebundenen DNA von Zellbestandteilen. Dabei wurde die Blutprobe zunächst lysiert. Danach folgte die Spaltung der Proteine mittels Proteinase K. Anschließend wurden dem Lysat magnetische Partikel beigefügt. Die DNA bindet aufgrund ihrer negativen Ladung an die Oberfläche dieser Partikel. Es folgte die magnetische Separation der DNA von den restlichen Zellkomponenten des Lysats. Abschließend wurde die DNA eluiert. Für die DNA-Extraktion fanden je $350 \mu \mathrm{L}$ der venösen Vollblutproben Verwendung. Des Weiteren kam der EZ1 DNA Blood $350 \mu \mathrm{L}$ Kit zum Einsatz. Alle Arbeitsschritte wurden gemäß des Herstellerprotokolls (EZ1 DNA Handbook 02/2004) durchgeführt. Als Produkte erhielt man je $200 \mu \mathrm{L}$ wässrige DNA-Lösungen unterschiedlicher Konzentration. Vor der Weiterverarbeitung wurden die DNA-Konzentrationen der Proben gemessen. Dies geschah mit Hilfe des Pico Green®-Reagenz, eines Farbstoffes, der bei Bindung an DNA bzw. Interkalation in DNA fluoresziert. Für die folgende PCR wurden die Proben einheitlich auf $10 \mathrm{ng}$ DNA/ $\mu \mathrm{L}$ Lösung eingestellt. 


\subsubsection{PCR und Fluoreszenzsignalerzeugung}

Die PCR dient der enzymatischen in-vitro-Amplifikation definierter DNA-Abschnitte. Der zu vervielfältigende Abschnitt wurde durch zwei spezifische Primer festgelegt. Primer sind synthetisch hergestellte Oligonukleotide, die auf den DNA-Einzelsträngen als Startpunkte für die DNA-Polymerase dienen. Der PCR-Prozess, bestehend aus etwa 25 bis 50 Zyklen, wurde in einem Thermocycler durchgeführt. Jeder Zyklus unterteilt sich typischerweise in drei Schritte. Zunächst wird die Doppelstrang-DNA auf $94-96^{\circ} \mathrm{C}$ erhitzt. Hierbei werden die Wasserstoffbrückenbindungen der Doppelhelix aufgespalten und es entstehen zwei Einzelstränge (Denaturierung). Damit sich die Primer den einzelnen DNA-Strängen anlagern können (Annealing), wird die Temperatur gesenkt $\left(50-70^{\circ} \mathrm{C}\right)$. Im letzten Schritt werden nun die DNA-Einzelstränge komplementär zu den DNA-Matrizen mit freien Nukleotiden synthetisiert. Meist wird dieser Prozess (Elongation) durch die hitzestabile Taq-Polymerase bei $72^{\circ} \mathrm{C}$ katalysiert. Jetzt liegt die DNA wieder als Doppelstrang vor und der nächste Zyklus kann sich anschließen. Als technische Grundlage der PCR diente ein vollautomatischer Thermocycler (Eppendorf Mastercycler $\circledast$, Eppendorf, Hamburg, Deutschland), der die verschiedenen Temperaturzyklen steuert. Jede Probe für den Cycler enthielt $1 \mu \mathrm{L}$ der zu Beginn extrahierten DNA (Konzentration: $10 \mathrm{ng}$ DNA/ $\mu \mathrm{L}$ Lösung), den Mastermix $(2.5 \mu \mathrm{L})$, einen für jeden SNP spezifischen TaqMan ${ }^{\circ}$ Sonden- und Primermix $(0.25 \mu \mathrm{L})$ und bidestilliertes Wasser $(1.25 \mu \mathrm{L}$ ). Der TaqMan $®$ Universal PCR Mastermix (Applied Biosystems, Carlsbad, USA) enthielt dNTPs, AmpliTaq®Gold-DNA-Polymerase, $\mathrm{MgCl}_{2}$ und Puffer.

TaqMan®-Sonden sind einzelsträngige Oligonukleotide, welche spezifisch für die möglichen Allele (nämlich *2, *3 bei CYP2C9 und *1 bei CYP3A5) sind. Während der PCR lagern sich die Sonden spezifisch an den innen komplementären Strang der DNA an. Am $5{ }^{\circ}$-Ende der Sonde befindet sich ein Fluoreszenzmarker (Reporter). Entsprechend der Allele handelt es sich hierbei um VIC oder FAM. Der am 3`-Ende lokalisierte Quencher unterdrückt (neutralisiert) das Fluoreszenzsignal des Reporters, wenn sich beide Moleküle, Reporter und Quencher, in einem definierten Abstand zueinander befinden. Nun binden die Primer und der Strang wird verlängert. Im Zuge der Amplifikation wird die fluoreszenzmarkierte Sonde vom Einzelstrang abgespalten. Dies geschieht durch die Taq-Polymerase, die zusätzlich zur Polymeraseaktivität auch eine $5^{`}-3^{\prime}$-Exonukleaseaktivität besitzt. Dadurch wird der 
definierte Abstand zwischen Reporter und Quencher aufgehoben, wodurch das Fluoreszenzsignal freigesetzt wird. Es bleibt auch nach der Fertigstellung der Doppelstränge bestehen. Das Signal wird umso stärker, je mehr DNA amplifiziert wird.

Dabei wurden die CYP2C9-Varianten Arg144Cys (Allel *2) und Ile359Leu (Allel*3) sowie der CYP3A5-Polymorphismus rs776746 (Allel *3) mit Hilfe der TaqMan®-SNPGenotypisierungs-Assays \#C_25625805_10, \#C_27104892_10 und \#C_26201809_30 typisiert (Applied Biosystems, Darmstadt, Germany). Die Sequenzen der Primer und Sonden wurden dabei vom Hersteller nicht angegeben.

Die OATP1B1 Polymorphismen Asn130Asp und Val174Ala wurden mittels DNASequenzierung typisiert. Dies erfolgte mit den Primern 5'-CAT GCT GGG AAA TTG ACA GAA AGT A-3' und 5'-TAC CGG CAG GTT TAT CAT CCA GTT C-3' für den Asn130Asp-Polymorphisus und mit den beiden Primern 5'-CCC CAG CCA TGA GGA ACT ATG AGT C-3' und 5'-TTT TCG CAT GTG TGC TTA GAA AGA C-3' für den Val174Ala-Polymorphismus. Die Sequenzierungsreaktion wurde jeweils von beiden Seiten mit den genannten Primern durchgeführt.

\subsubsection{Allelische Diskriminierung}

Die durch die PCR amplifizierten Proben wurden zur allelischen Diskriminierung in das 7900 HAT Sequence Detection System (Applied Biosystems, Carlsbad, USA) gegeben. Hier wurde die Fluoreszenz der freigesetzten Marker (VIC und FAM) mit Laserlicht aus einem Argon-Laser angeregt. Emissionen zwischen 500 und 660 nm wurden mit einer Kamera aufgenommen. Da die Marker bei unterschiedlichen Wellenlängen ihr Maximum haben, kann man über die Quantifizierung der VIC- und FAM-Emissionen auf das Vorkommen des jeweiligen Allels schließen. 


\subsection{Konzentrationsbestimmung des THC und seiner Metabolite im Plasma}

Die Plasmakonzentrationen von Delta-9-Tetrahydrocannabinol (THC), 11-HydroxyDelta-9-THC (OH-THC) und 11-Nor-9-Carboxy-Delta-9-Tetrahydrocannabinol ( $\mathrm{COOH}-\mathrm{THC})$ wurden mittels einer aus der Literatur adaptierten Methode bestimmt (Maralikova und Weinmann 2004). Die bei der Analytik verwendeten Substanzen wurden in Methanol gelöst von der Firma Promochem (Wesel, Deutschland) bezogen. Die Messung der Plasmaproben erfolgte durch Flüssigkeitschromatographie mit gekoppelter Massenspektrometrie (LC-MS) durch das Perkin-Elmer/API 4000 triple quadruple instrument (Applied Biosystems, Carlsbad, USA). Zur Messung der Plasmaproben wurde $1 \mathrm{~mL}$ Aliquot mit $25 \mu \mathrm{L}$ der Internen Standards versetzt (d3-Analoge der Analyte THC, OH-THC und $\mathrm{COOH}-\mathrm{THC}$ in einer Konzentration von $200 \mathrm{ng} / \mathrm{mL}$ für THC und $\mathrm{OH}-\mathrm{THC}$ sowie $400 \mathrm{ng} / \mathrm{mL}$ für $\mathrm{COOH}-\mathrm{THC}$ ). Anschließend wurden $1.5 \mathrm{~mL} 1.0$ molare Essigsäure zugegeben. Nun wurde die Probe in eine konditionierte Chromabond C18 Kartusche (500 mg Sorptionsmittel, Macherey\&Nagel, Düren, Deutschland) überführt. Die Kartusche wurde dann mit je $1 \mathrm{~mL} 0.1$ molarer Essigsäure und einer Mischung aus Acetonitril und Wasser $(70 / 30, v / v)$ gewaschen. Es folgte die Elution der Analyten aus der Kartusche mittels $2 \mathrm{~mL}$ Acetonitril. Bei einer Temperatur von $40^{\circ} \mathrm{C}$ wurde das Lösungsmittel durch die Bedampfung mit Stickstoff entfernt. Der Rückstand wurde in $100 \mu \mathrm{L}$ mobiler Phase für die HPLC gelöst. Die mobile Phase bestand aus einem Acetat-Puffer mit einem pH-Wert von 4 (10 millimolare Essigsäure und 2.5 millimolares Ammoniumacetat) und Methanol (20/80, v/v). $10 \mu \mathrm{L}$ der Proben wurden in die HPLC injiziert. Die Trennung erfolgte mittels einer XTerra ${ }^{\text {TM }}$-Säule $(50 \times 2.1 \mu \mathrm{mm}, 3.5 \mu \mathrm{m}$; Waters, Eschborn, Deutschland) bei einem Fluss von $0.3 \mathrm{~mL} / \mathrm{min}$. Unter diesen Konditionen wurden Retentionszeiten von $1.8 \mathrm{~min}, 2.0 \mathrm{~min}$ und $4.1 \mathrm{~min}$ für $\mathrm{OH}-\mathrm{THC}, \mathrm{COOH}-\mathrm{THC}$ und THC beobachtet. Die Detektion der Analyten erfolgte nach Elektro-Spray-Ionisation (electro spray ionisation) durch Vielfach-lonen-Registrierung (multiple ion monitoring). Das Molekular-Ion von THC $(315 \mathrm{~m} / \mathrm{e})$ wurde als Vorläufer (precursor) verwendet. Quantifiziert wurde das Molekülfragment 259 m/e. Für $\mathrm{OH}-\mathrm{THC}$ und $\mathrm{COOH}-\mathrm{THC}$ wurden die Paare 331/313 m/e bzw. 345/327 m/e verwendet. Die Quantifizierung erfolgte anhand von Kalibrierungsproben im Messbereich von 0.2 bis $10 \mathrm{ng} / \mathrm{mL}$ für THC und $\mathrm{OH}-\mathrm{THC}$ und 0.4 bis $20 \mathrm{ng} / \mathrm{mL}$ für $\mathrm{COOH}-\mathrm{THC}$. Dazu wurden die 
normierten Messwerte (Peakflächen der Analyten dividiert durch Peakflächen der internen Standards) als Funktion der Konzentration einer Regressionsanalyse unterzogen. Die Ergebnisse der Konzentrationsanalysen wurden in $\mathrm{ng} / \mathrm{mL}$ ausgegeben. Die Analyten lagen dabei als freie Basen vor.

Proben, die Konzentrationen oberhalb von $10 \mathrm{ng} / \mathrm{mL}$ THC und OH-THC sowie oberhalb von $20 \mathrm{ng} / \mathrm{mL} \mathrm{COOH}-\mathrm{THC}$ aufwiesen, wurden nach Verdünnung mit Leerwertplasma erneut gemessen. Das Quantifizierungslimit dieser Methode lag bei $0.2 \mathrm{ng} / \mathrm{mL}$. Es ergab sich ein Variationskoeffizient von 16\%. In jeder Messreihe waren drei Proben zur Qualitätskontrolle inbegriffen, gemessen bei 1.5 und $8 \mathrm{ng} / \mathrm{ml}$ für THC und $\mathrm{OH}-\mathrm{THC}$ sowie 2, 10 und $16 \mathrm{ng} / \mathrm{mL}$ für $\mathrm{COOH}-\mathrm{THC}$. Die Messgenauigkeit (inter-day Variationskoeffizient) dieser Proben war für THC 4\%, 4\% und $5 \%(n=23)$, für $\mathrm{OH}-\mathrm{THC} 5 \%, 3 \%$ und $3 \%(n=23)$ und für $\mathrm{COOH}-\mathrm{THC} 4 \%, 5 \%$ und $5 \%(n=23)$.

\subsection{Konzentrationsbestimmung von $\mathrm{COOH}-\mathrm{THC}$ im Urin}

Der THC-Metabolit 11-Nor-9-Carboxy-Delta-9-Tetrahydrocannabinol (COOH-THC) wird als Glukuronid im Urin ausgeschieden. Die Muttersubstanz THC und der hydroxylierte Metabolit finden sich im Urin nur in geringen Spuren und wurden auch in der vorliegenden Studie nie oberhalb der Quantifizierungsgrenze von $0.4 \mathrm{ng} / \mathrm{mL}$ nachgewiesen.

Die Urinkonzentration von $\mathrm{COOH}-\mathrm{THC}$ wurde nach demselben Prinzip wie die Konzentrationsmessungen der Blutplasmaproben analysiert [Flüssigkeitschromatographie mit gekoppelter Massenspektrometrie (LC-MS)]. Da der CarboxyMetabolit des THC in glukuronidierter Form in den Urin ausgeschieden wird, musste zuerst die Deglukuronidierung zum $\mathrm{COOH}-\mathrm{THC}$ durchgeführt werden. Dafür wurden $1000 \mu \mathrm{L}$ der Urinprobe mit $300 \mu \mathrm{L}$ Ammoniumacetat-Puffer (250 mM; pH 5.0) und $25 \mu \mathrm{L}$ des Internen Standards (d3-Analoge der Analyten) versetzt. Danach erfolgte die Zugabe von $50 \mu \mathrm{L}$ Glukuronidase-Lösung (200 U/50 $\mu \mathrm{L}$ ). Die Glukuronidase wurde von der Firma Sigma-Aldrich (St. Louis, USA) bezogen. Es handelte sich um Glukuronidase der Helix pomatia, Typ H-2. Anschließend wurde das Stoffgemisch für 19 Stunden bei $37^{\circ} \mathrm{C}$ inkubiert. Die weitere Aufarbeitung und Messung der Proben wurde nach dem Protokoll der Messungen von $\mathrm{COOH}-\mathrm{THC}$ im Blutplasma vorgenommen. Die Quantifizierung des $\mathrm{COOH}-\mathrm{THC}$ erfolgte anhand von 
Kalibrierungsproben im Messbereich 0.4 bis $60 \mathrm{ng} / \mathrm{mL}$. Proben, die Konzentrationen oberhalb von $60 \mathrm{ng} / \mathrm{mL}$ aufwiesen, wurden nach Verdünnung mit Leerwerturin erneut gemessen. Das Quantifizierungslimit dieser Methode war $0.4 \mathrm{ng} / \mathrm{mL}$. Bei dieser Konzentration ergab sich ein Variationskoeffizient von 13\%. In jeder Messreihe waren drei Proben zur Qualitätskontrolle inbegriffen. Hierbei wurde jeweils eine Urinprobe eines Probanden verwendet, und zwar nach Vormessungen eine Probe mit niedriger, mittlerer und hoher Konzentration von $\mathrm{COOH}-\mathrm{THC}$. Die Verwendung des kommerziell bezogenen reinen THC bzw. COOH-THC war hier nicht geeignet, da die Qualitätskontrollen auch die Vollständigkeit der Glukuronidase-Spaltung mit erfassen sollten. Es ergaben sich bei den drei Kontrollen Mittelwerte $(n=18)$ von 1.21, 15.0 und $41.4 \mathrm{ng} / \mathrm{mL}$. Die dementsprechenden Variationskoeffizienten (Mittelwert/Standardabweichung-100) waren $22.6 \%, 14.7 \%$ und $13.2 \%$.

\subsection{Pharmakokinetische Auswertungen und Statistik}

\subsubsection{Pharmakokinetische Begriffe und Parameter}

Wie bereits dargestellt, wurden nach Applikation der Studienmedikation je Proband zu elf Zeitpunkten Blutplasmakonzentrationen von THC und seinen Hauptmetaboliten, $\mathrm{OH}-\mathrm{THC}$ und $\mathrm{COOH}-\mathrm{THC}$, bestimmt. Gleichwohl wurden fünf Urinfraktionen gesammelt. Die entsprechenden Messwerte wurden zu wesentlichen pharmakokinetischen Kenngrößen (Parameter) zusammengefasst.

Ein Parameter, der sich besonders zur Charakterisierung der Pharmakokinetik eines Arzneistoffs eignet, ist die AUC. Sie stellt die Gesamt-Exposition des Körpers gegenüber dem Medikament dar. Trägt man die nach Medikamentenapplikation gemessenen Plasmakonzentrationen gegen die Zeit auf, so entspricht die Fläche unter der resultierenden Konzentrations-Zeit-Kurve der AUC. Anders ausgedrückt ist sie als Integral der Konzentrationen über die Zeit definiert. Die AUC ermöglicht die Berechnung der totalen Clearance eines Medikaments. Hierbei handelt es sich um einen pharmakokinetischen Parameter zur Charakterisierung und Quantifizierung der Eliminationsprozesse. Die Clearance gestattet es, die Eliminationsgeschwindigkeit eines Pharmakons bei gegebener Plasmakonzentration zu berechnen. Die totale Clearance setzt sich aus der renalen und der extrarenalen Clearance zusammen. 
Die extrarenale Clearance beschreibt alle nichtrenalen Eliminationsvorgänge wie beispielsweise den Metabolismus in der Leber.

Der Begriff der Bioverfügbarkeit (F) charakterisiert die Bereitstellung eines Pharmakons für systemische, also den ganzen Körper betreffende Wirkungen. Sie berechnet sich nach der Formel $\mathrm{F}=\mathrm{AUC}_{\mathrm{p.o.}} / \mathrm{AUC}_{\mathrm{i} . \mathrm{v} . \text {. }}$.

Weitere bedeutende Zielgrößen sind die Spitzenkonzentration $\left(\mathrm{C}_{\max }\right)$, der Zeitpunkt der maximalen Blutkonzentration $\left(t_{\max }\right)$, die Plasmakonzentration, 24 Stunden nach Applikation $\left(\mathrm{C}_{24 \mathrm{~h}}\right)$ sowie die terminale Halbwertszeit $\left(\mathrm{t}_{1 / 2}\right)$ und das Verteilungsvolumen $(\mathrm{V})$.

Bei der Spitzenkonzentration $\left(C_{\max }\right)$ handelt es sich um die maximal gemessene Plasmakonzentration der Einzelkinetik. Dieser Wert ist im Wesentlichen vom Verteilungsvolumen eines Medikaments abhängig, in geringerem Umfang auch von der Clearance. Als Zeitpunkt der maximalen Blutkonzentration $\left(t_{\max }\right)$ wird der jeweilige Zeitpunkt nach Medikamentenapplikation angegeben, zu dem $\mathrm{C}_{\max }$ bestimmt wurde. Das 24-h-trough-level $\left(\mathrm{C}_{24 \mathrm{~h}}\right)$ gibt die Stoffkonzentration im Blutplasma an, die 24 Stunden nach Medikamentenapplikation gemessen wurde. Die Halbwertszeit $\left(\mathrm{t}_{1 / 2}\right)$ ist die Zeitspanne bis zum Abfall der Ausgangskonzentration auf die Hälfte des ursprünglichen Wertes. Sie hängt sowohl von der Eliminationsleistung des Organismus, also von der Clearance, als auch vom Verteilungsvolumen eines Pharmakons ab. Dabei ist die Halbwertszeit umso länger, je größer das Verteilungsvolumen, und umso kürzer, je größer die Clearance ist. Häufig ist eine Summe von zwei oder mehr Exponentialfunktionen nötig, um den Zeitverlauf der Plasmakonzentration eines Pharmakons zu beschreiben. Unter der Annahme einer Zwei-Phasen-Kinetik für THC (Nadulski et al. 2005) ergibt sich aus der Phase des schnellen Konzentrationsabfalls (Alpha-Phase) die dominierende und aus der Phase des langsamen Konzentrationsabfalls (Beta-Phase) die terminale Halbwertszeit. Als dominierende Halbwertszeit wird die Halbwertszeit derjenigen Phase bezeichnet, die am meisten zur AUC beiträgt. Oftmals ist die terminale mit der dominierenden Halbwertszeit gleichzusetzen. Das Verteilungsvolumen (V) ist definitionsgemäß ein Proportionalitätsfaktor zwischen der im Organismus vorhandenen Menge (M) eines Pharmakons und seiner Plasmakonzentration (c) und wird als Quotient aus M und c berechnet. Je größer das Verteilungsvolumen ist, desto kleiner ist die Plasmakonzentration im Vergleich zur im Körper befindlichen 
Gesamtarzneistoffmenge. Ähnlich wie bei der Clearance kann das Verteilungsvolumen bei oraler Dosierung nur in Relation zur Bioverfügbarkeit angegeben werden (V/F). Die genaue Bestimmung erfordert eine intravenöse Applikation.

\subsubsection{Bestimmung pharmakokinetischer Parameter}

Die Ergebnisse der Plasmakonzentrationsmessungen wurden mit Hilfe der Computersoftware WinNonlin ${ }^{\mathrm{TM}}$ (Pharsight Corporation, Mountain View, USA) analysiert.

Zur Berechnung der AUC wurde mittels der Trapezregel $[(\mathrm{C} 1+\mathrm{C} 2) / 2 \cdot(\mathrm{t} 2-\mathrm{t} 1)]$ die Fläche bis zur letzen Messung bestimmt. Anschließend wurde die Restfläche vom letzen Messzeitpunkt an entsprechend dem Konzentrationsabfall der letzten drei gemessen Konzentrationen gegen unendlich extrapoliert $\left(A \cup C_{0 \text {-unendlich }}\right)$. Die Einheit war $\mu \mathrm{g} \cdot \mathrm{h} / \mathrm{L}$. Das Prinzip der totalen Clearance ist nur bei intravenöser Dosierung anwendbar und errechnet sich nach der Formel $\mathrm{Cl}_{\text {total }}=$ Dosis $/ \mathrm{AUC}_{0 \text {-unendlich. }} \mathrm{Im}$ Falle der oralen Medikamentengabe lässt sich lediglich die orale Clearance angeben. Sie errechnet sich aus dem Quotienten von Clearance und Bioverfügbarkeit ( $\mathrm{Cl} / \mathrm{F})$ und schließt neben der systemischen auch mögliche präsystemische Clearanceanteile mit ein. Da die Menge von THC, die zum jeweiligen Metaboliten verstoffwechselt wurde, unbekannt war und es damit nicht möglich war, eine Aussage bezüglich der Quantität der Metabolite zu treffen, ließ sich die Clearance lediglich für das THC selbst bestimmen, nicht aber für die Metaboliten $\mathrm{OH}-\mathrm{THC}$ und $\mathrm{COOH}-\mathrm{THC}$. Die Bioverfügbarkeit wurde aus Literaturdaten entnommen (Grotenhermen 2003). Bei den Angaben für die maximalen Blutkonzentrationen $\left(C_{\max }\right)$ fanden jeweils die Werte wie gemessen Verwendung, d.h. es wurde keine Extrapolation oder Interpolation durchgeführt. Die Maßeinheit war $\mu \mathrm{g} / \mathrm{L}$. Der Zeitpunkt der maximalen Blutkonzentration $\left(\mathrm{t}_{\max }\right)$ wurde in Stunden angegeben. Das 24-h-trough-level $\left(\mathrm{C}_{24 \mathrm{~h}}\right)$ wurde direkt gemessen. Die Einheit war $\mu \mathrm{g} / \mathrm{L}$. Die terminale Halbwertszeit wurde aus dem Abfall der letzten drei Konzentrationen ermittelt. Dazu wurde durch die logarithmierten Blutkonzentrationen eine Regressionsgerade gelegt. Der Abfall dieser Regressionsgeraden entspricht der so genannten Eliminationskonstanten $\left(\mathrm{K}_{\mathrm{el}}\right)$. Die terminale Halbwertszeit wurde als Quotient des natürlichen Logarithmus von zwei und der Eliminationskonstanten berechnet $\left(t_{1 / 2}\right)=\ln (2) / K_{e l}$. Der natürliche 
Logarithmus von zwei ist gleich 0.693. Die Halbwertszeit wurde in Stunden (h) ausgegeben.

\subsubsection{Statistische Analysen}

\subsubsection{Fallzahl und Zielgrößen}

Die AUCs von THC, OH-THC und $\mathrm{COOH}-\mathrm{THC}$ wurden im Vorfeld der Studie als primäre Zielparameter festgelegt. Da bei THC selbst die totale Clearance der Dosis dividiert durch die AUC entsprach, kann die totale THC-Clearance gleichermaßen als primäre Zielgröße angesehen werden. Jedoch konnte, wie bereits erwähnt, hier nur die orale Clearance $(C / / F)$ bestimmt werden, da für die Berechnung der totalen Clearance die intravenöse Applikation des Studienmedikaments nötig gewesen wäre.

Das Ziel war, die Abhängigkeit der genannten Parameter von der Zahl an CYP2C9*1-, -*2- und -*3-Allelen sowie von der Zahl der CYP3A5*1- und -*3-Allele zu untersuchen. Bezogen auf diese Analysen wurde keine Adjustierung auf multiples Testen durchgeführt.

Basierend auf einer früheren Studie mit einer durchschnittlichen AUC (Standardabweichung) von 1020 (320) $\mu \mathrm{g} \cdot \mathrm{min} / \mathrm{L}$ (Ohlsson et al. 1980) wurde, um einen 50\%igen Unterschied zu erbringen (beispielsweise 1530 anstelle von 1020), mit dem Programm S-Plus 6.2 (Insightful Corp, Seattle, USA) eine minimale Gruppengröße von $n=7$ kalkuliert. Dabei wurde von einem Alpha-Fehler von 0.05 (Typ-I-Fehler, Risiko falsch positiver Befunde) und einem Beta-Fehler von 0.2 (Typ-II-Fehler, Risiko falsch negativer Befunde) ausgegangen. Diese Gruppengröße wurde bei den heterozygoten Genotypen angestrebt. Bei den seltenen homozygoten Genotypen sollte eine größtmögliche Anzahl von Probanden eingeschlossen werden. Die Stichprobe wurde also so berechnet, dass mindestens 7 Personen mit den Genotypen CYP2C9*1/*1, *1/*2 und *1/*3 vorliegen sollten. Darüber hinaus sollte die Bedeutung der CYP3A5-Variante *1 (enzymatisch aktiv) versus *3 (enzymatisch nicht aktiv) geprüft werden. Hier war geplant, in der hinsichtlich des Enzyms CYP2C9 größten Gruppe $\left(C Y P 2 C 9 * 1 /{ }^{\star} 1\right)$ nach oben genannten Überlegungen ebenfalls mindestens je 7 Personen mit $C Y P 3 A 5 * 1 /{ }^{*} 1$ und $C Y P 3 A 5^{\star} 1 /{ }^{*} 3$ zu untersuchen. 


\subsubsection{Exploratorische Datenanalyse}

Die weitere Präsentation und Analyse der Daten beinhaltete deskriptive exploratorische Datenanalysen sowie testende Statistik. Im Rahmen der graphischen Präsentation wurden in vielen Fällen die Einzelwerte gezeigt, zum Beispiel auch die einzelnen Konzentrationsverläufe, nicht zuletzt um zu illustrieren, wie die Variation zwischen den Probanden war. Darüber hinaus wurden so genannte Box-Plots (bzw. Box-and-whisker-Plots) verwendet. Diese wurden mit dem Programm SPSS 12.0 für Windows (SPSS Inc., Chicago, USA) produziert. Dabei zeigt der Box-Plot einen Kasten (Box), der unten und oben von der 1. und 3. Quartile begrenzt ist und in dem der Median als kräftige Linie gezeigt ist. Die Differenz aus oberem und unterem Quartil wird als Interquartilsabstand (interquartile range) bezeichnet. Die Antennen der Box-Plots (Whisker) sind berechnet als Median +/- 1.5 mal des interquartilen Bereiches. Darüber hinaus sind Ausreißer (Kreise) und weite Ausreißer (Sterne) definiert, je nachdem wie weit sie vom Interquartilen-Bereich abweichen.

\subsubsection{Nichtparametrische Tests}

In der Analyse, ob Unterschiede in metrischen Größen, wie Körpergewicht, Clearance oder Halbwertzeit statistisch signifikant sind, gibt es zwei unterschiedliche Testverfahren. Zum einen sind dies die so genannten nichtparametrischen Tests, die auch bei Werten angewendet werden können, die nicht normalverteilt sind, zum anderen gibt es so genannte parametrische Tests, die formal nur bei normalverteilten Werten gültig sind. Sie werden allerdings von vielen Autoren auch angewendet, wenn eine Normalverteilung nicht streng erfüllt ist. Aus dem praktischen Grund heraus, dass es unter den multifaktoriellen Analysen, die gemeinsam den Einfluss vieler Faktoren darstellen, mehr parametrische Tests gibt, wurden hier, gerade im Bereich multifaktorieller Analysen, auch parametrische Tests verwendet.

Als nichtparametrische Kenngrößen wurden der Median, der Interquartilsabstand als Bereich zwischen 1. und 3. Quartile bzw. 25. und 75. Perzentile sowie der Bereich (kleinster bis größter Wert) verwendet. Als nichtparametrische Tests für den Vergleich von zwei Gruppen fand der Man-Whitney-U-Test Anwendung. Bei Mehrgruppenvergleichen gab es in der Regel eine vor Studienbeginn klar definierte Ordnung der Gruppen, so dass hier der nichtparametrische Jonckheere-TerpstraTrend-Test angewendet wurde. Zur statistischen Testung war, basierend auf 
früheren Studien, durch das Studienprotokoll die Reihenfolge der CYP2C9Genotypen wie folgt vordefiniert: CYP2C9*1/*1, ${ }^{*} 1 /{ }^{*} 2,{ }^{*} 2 /{ }^{*} 2,{ }^{*} 1 /{ }^{*} 3,{ }^{*} 2 /{ }^{*} 3$ und ${ }^{*} 3 /{ }^{*} 3$. Wenn nur eines der varianten Allele betrachtet wurde, war die Reihenfolge dementsprechend beispielsweise 0,1 , und 2 CYP2C9*3-Allele.

Die Analyse des Alles CYP3A5*1/*3 verglichen mit dem Allel CYP3A5*3/*3 basierte auf Probanden des Genotyps CYP2C9*1/*1. Folglich wurden insgesamt folgende Genotypen einander gegenübergestellt: CYP2C $9^{*} 1 /{ }^{*} 1-C Y P 3 A 5^{\star} 1 /{ }^{*} 3, C Y P 2 C{ }^{*} 1{ }^{*} 1-$ CYP $3 A{ }^{*} 3 / * 3, \quad C Y P 2 C 9 * 1 / * 2, C Y P 2 C 9 * 2 / * 2, C Y P 2 C 9 * 1 / * 3, C Y P 2 C 9 * 2 /{ }^{*} 3$ sowie CYP2C9*3/*3. Der CYP3A5-Genotyp der letzten fünf CYP2C9-Genotypen war vor Studienbeginn nicht bekannt, wurde aber im Zuge der Studie ebenfalls mitbestimmt.

\subsubsection{Parametrische statistische Analyse}

Zusätzlich zu den nichtparametrischen Analysen erfolgten univariate Varianzanalysen, um mögliche sich ergänzende oder addierende Effekte zwischen Geschlechtern, CYP2C9-Genotypen, dem CYP3A5-Genotyp, dem OATP1B1Genotyp sowie Körpergewicht und Serumalbumin zu untersuchen.

Im Rahmen der Datenpräsentation wurde in vielen Fällen auch der Mittelwert mit Standardabweichung angegeben. Dies geschah nicht zuletzt, um unsere Werte mit der Literatur vergleichbar zu halten. Als Analyseverfahren wurde ferner die multiple lineare Regressionsanalyse verwendet. 


\section{Ergebnisse}

\subsection{Untersuchte Probanden und deren molekulargenetische Daten (Charakteristika der Probandenkohorte)}

In unserer Studie ging es darum, bei gesunden Probanden die Bedeutung von Cytochrom-P450-Genvarianten für die Pharmakokinetik und die Wirkungen von Tetrahydrocannabinol zu untersuchen. Dies wurde an 43 gesunden Kaukasiern, das heißt Personen europäischer Abstammung, untersucht. Davon waren 16 weiblichen und 27 männlichen Geschlechts (37.2\% Frauen zu 62.8\% Männer). Ein geplanter Anteil weiblicher Probanden von $50 \%$ in jeder der Untergruppen konnte nicht erreicht werden. Einerseits gab es weniger Interessentinnen als Interessenten, andererseits konnten viele Frauen die Einschlusskriterien nicht erfüllen. Der häufigste Ausschlussgrund war die Einnahme hormoneller Kontrazeptiva. Außerdem ist zu berücksichtigen, dass weniger als $1 \%$ der Bevölkerung den für unsere Studie besonders interessanten Genotyp CYP2C9*3/*3 tragen und auch der heterozygote Genotyp CYP2C9*1/*3 in der unselektierten Gruppe nur mit einer Wahrscheinlichkeit von etwa $12 \%$ vorliegt. Dementsprechend konnte auch eine balancierte Aufteilung nach Geschlechtern innerhalb dieser Genotyp-Untergruppen nicht immer erreicht werden.

Tabelle 8 zeigt die demographischen und molekulargenetischen Daten aller Probanden. Dargestellt sind Mittelwert und Standardabweichung sowie der Bereich vom Minimum zum Maximum. Ebenfalls ist die Auftrennung nach CYP2C9- und CYP3A5-Genotypen dargestellt.

Das mittlere Alter betrug $27.3 \pm 7.8$ Jahre (Mittelwert \pm Standardabweichung). Die Probanden wiesen bei einem mittleren Körpergewicht von $71.6 \pm 10.3 \mathrm{~kg}$, einen mittleren BMl von $23.0 \pm 2.2$ auf. 
Tab. 8 Demographische Daten der Studiengruppe unter $\mathrm{n}$ Probanden

\begin{tabular}{|c|c|c|c|c|c|c|c|}
\hline & Total & $\begin{array}{c}\% \text { von } \\
\text { Total }\end{array}$ & Männer & Frauen & Alter & $\begin{array}{l}\text { Körper- } \\
\text { gewicht }\end{array}$ & $\begin{array}{c}\text { Körper- } \\
\text { oberfläche }\end{array}$ \\
\hline & $\mathrm{n}$ & & $\mathrm{n}$ & $\mathrm{n}$ & \multicolumn{3}{|c|}{$\begin{array}{c}\text { Mittelwert (Standardabweichung) } \\
\text { Bereich }\end{array}$} \\
\hline Gesamt & 43 & 100 & 27 & 16 & $\begin{array}{c}27.3(7.8) \\
20-65\end{array}$ & $\begin{array}{l}71.6(10.3) \\
54-94\end{array}$ & $\begin{array}{c}1.87(0.16) \\
1.6-2.2\end{array}$ \\
\hline $\begin{array}{c}\text { CYP } 3 A 55 \\
* 3 / * 3\end{array}$ & 34 & 79.1 & 23 & 11 & $\begin{array}{c}27.8(8.6) \\
20-65\end{array}$ & $\begin{array}{l}71.6(10.7) \\
54-94\end{array}$ & $\begin{array}{c}1.88(0.16) \\
1.6-2.2\end{array}$ \\
\hline $\begin{array}{l}\text { CYP3A5 } \\
* 1 / * 3\end{array}$ & 9 & 20.9 & 4 & 5 & $\begin{array}{c}24.9(2.4) \\
22-29\end{array}$ & $\begin{array}{c}71.7(9.9) \\
54-87\end{array}$ & $\begin{array}{c}1.86(0.16) \\
1.6-2.1\end{array}$ \\
\hline $\begin{array}{l}\text { CYP2C9 } \\
{ }^{*} 1 /{ }^{*} 1\end{array}$ & 19 & 47.5 & 12 & 7 & $\begin{array}{c}26.2(4.8) \\
20-42\end{array}$ & $\begin{array}{c}73.5(9.5) \\
60-91\end{array}$ & $\begin{array}{c}1.90(0.15) \\
1.6-2.1\end{array}$ \\
\hline $\begin{array}{l}\text { CYP2C9 } \\
* 1 / * 2\end{array}$ & 6 & 15.0 & 3 & 3 & $\begin{array}{c}24.2(2.1) \\
22-28\end{array}$ & $\begin{array}{l}71.0(12.5) \\
55-89\end{array}$ & $\begin{array}{c}1.87(0.19) \\
1.6-2.1\end{array}$ \\
\hline $\begin{array}{l}\text { CYP2C9 } \\
* 2 / * 2\end{array}$ & 4 & 5.0 & 3 & 1 & $\begin{array}{l}38.8(19.2) \\
23-65\end{array}$ & $\begin{array}{c}70.4(12.1) \\
55-84\end{array}$ & $\begin{array}{c}1.85(0.17) \\
1.6-2.0\end{array}$ \\
\hline $\begin{array}{l}\text { CYP2C9 } \\
{ }^{*} 1 /{ }^{*} 3\end{array}$ & 9 & 22.5 & 6 & 3 & $\begin{array}{c}26.3(4.9) \\
22-38\end{array}$ & $\begin{array}{c}70.2(11.5) \\
54-94\end{array}$ & $\begin{array}{c}1.87(0.17) \\
1.6-2.2\end{array}$ \\
\hline $\begin{array}{c}\text { CYP2C9 } \\
* 2 / * 3\end{array}$ & 1 & 2.5 & 1 & 0 & 22.0 & 79.0 & 2.0 \\
\hline $\begin{array}{c}\text { CYP2C9 } \\
* 3 / * 3\end{array}$ & 4 & 7.5 & 2 & 2 & $\begin{array}{c}28.5(7.7) \\
21-39\end{array}$ & $\begin{array}{c}66.0(11.8) \\
58-78\end{array}$ & $\begin{array}{c}1.80(0.18) \\
1.6-2.0\end{array}$ \\
\hline
\end{tabular}

Alter in Jahren, Körpergewicht in kg, Körperoberfläche in $\mathrm{m}^{2}, \mathrm{n}=$ Anzahl der Probanden

Alle Studienteilnehmer erfüllten die entsprechenden Einschlusskriterien. Unter den Probanden gab es hinsichtlich der demographischen Daten keine signifikanten Unterschiede zwischen den Gruppen mit den Genotypen von CYP2C9 und CYP3A5. Der erste Proband erhielt $30 \mathrm{mg}$ Dronabinol, der zweite Studienteilnehmer nahm 10 mg der Studienmedikation ein. Unter Berücksichtigung einer zur Dosis proportionalen (so genannten linearen) Pharmakokinetik wurden die Konzentrationen der ersten beiden Probanden auf eine Dosis von $15 \mathrm{mg}$ normiert. Allen folgenden 41 Probanden wurden $15 \mathrm{mg}$ Dronabinol verabreicht. Das Studienmedikament Dronabinol wurde insgesamt gut vertragen. Eine Nebenwirkung ist nach internationalen Kriterien als schwerwiegend einzustufen, wenn sie tödlich oder lebensbedrohend ist, eine stationäre Behandlung oder Verlängerung einer stationären Behandlung erforderlich macht oder zu bleibender oder schwerwiegender Behinderung oder Invalidität führt. In unserer Studie traten keine schwerwiegenden unerwünschten Ereignisse (adverse events) auf. Kein Proband musste nach Beginn 
aufgrund Nichteinhaltens der Studienordnung ausgeschlossen werden. Sämtliche Probanden wurden in die Analysen einbezogen.

\subsection{Pharmakokinetische Daten und CYP2C9-Genotyp}

\subsubsection{Konzentrations-Zeit-Verläufe von THC}

Den Probanden wurde zu den in Kapitel 2.1.7 vordefinierten Zeitpunkten nach Medikamentenapplikation Blut entnommen. Die Konzentrationen von THC und seinen Metaboliten wurden im Blutplasma mittels HPLC und massenspektrometrischer Detektion analysiert. Die Konzentrations-Zeit-Verläufe des THC aller Probanden sind in Abbildung 4 dargestellt. Es ist zu erkennen, dass insgesamt eine deutliche Streuung der Kurvenverläufe unter den Probanden bestand. Ebenso wird im Hinblick auf den Abfall der Plasmakonzentration von THC deutlich, dass es anfänglich zu einem rascheren und anschließend zu einem langsameren Absinken der Plasmakonzentration kam.

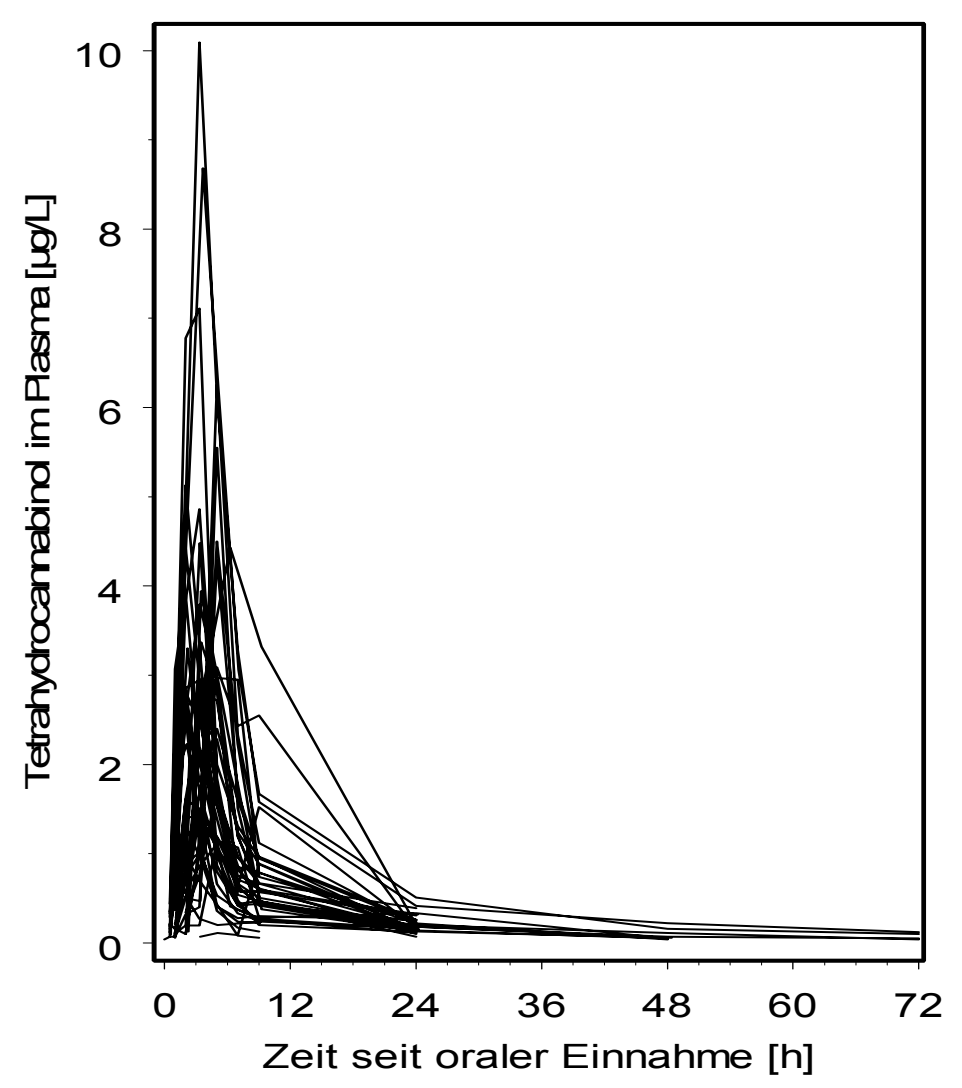

Abb. 4 Illustration der Streuung der Konzentrations-Zeit-Verläufe von THC im Blutplasma aller Probanden 
Der biexponentielle Konzentrationsabfall verdeutlicht sich, wie in Abbildung 5 gezeigt, bei halblogarithmischer Darstellung. Es ergab sich also eine Mehrphasenkinetik für THC, weswegen sich die Beschreibung des KonzentrationsZeit-Verlaufs von THC aus der Summe mehrerer Exponentialfunktionen zusammensetzt. Zur weiteren Analyse dieses biexponentiellen Konzentrationsverlaufes wurde eine pharmakokinetische Modellanpassung an ein 2-Kompartiment-Modell durchgeführt und die entsprechenden pharmakokinetischen Parameter bestimmt (Kapitel 3.3).

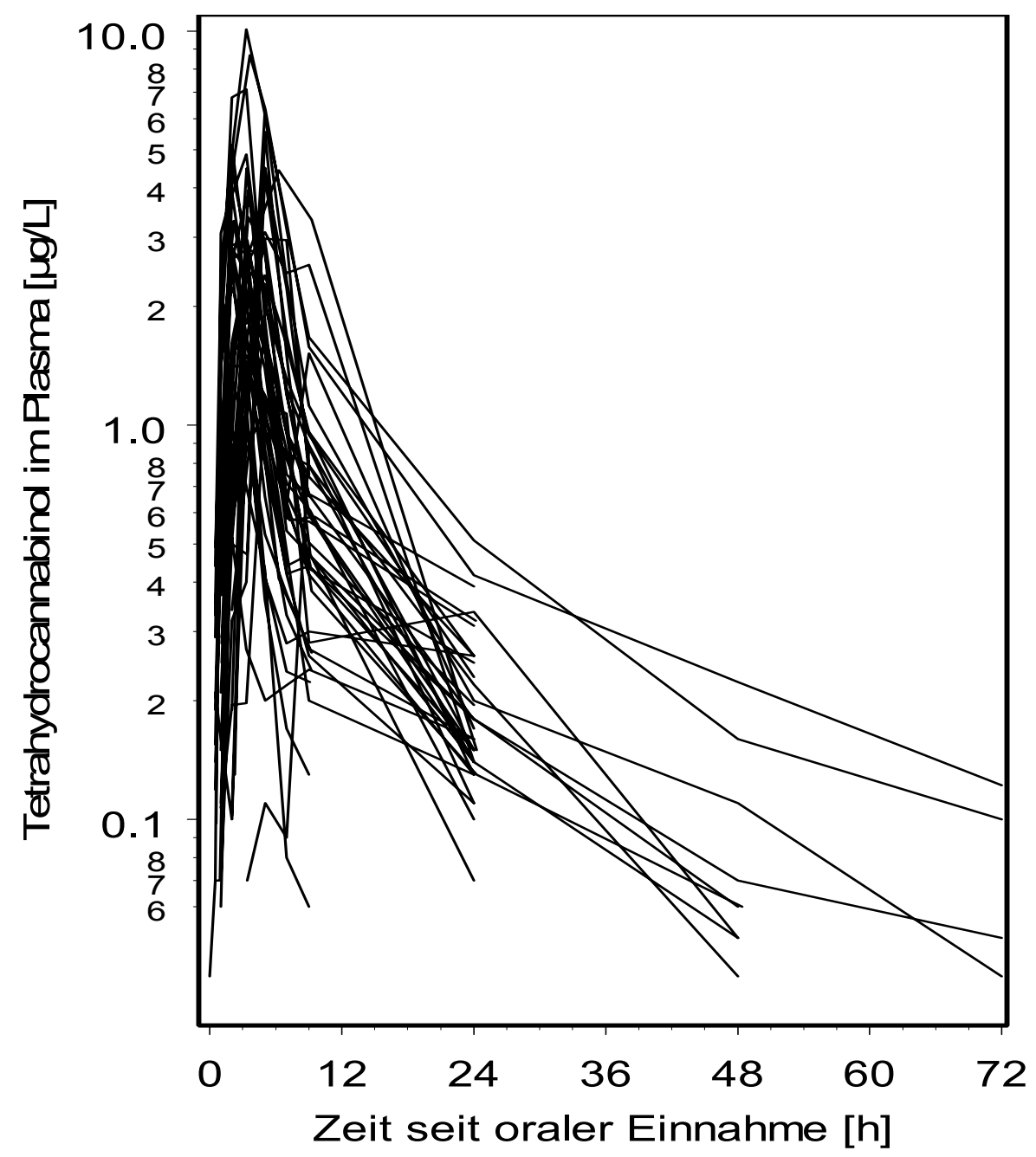

Abb. 5 Halblogarithmische Darstellung der Konzentrations-Zeit-Verläufe von THC im Blutplasma aller Probanden 


\subsubsection{Pharmakokinetische Parameter von THC}

In der Pharmakokinetik werden die Konzentrationsmessungen zu charakteristischen Kenngrößen (pharmakokinetische Parameter) zusammengefasst. Die pharmakokinetischen Parameter von Delta-9-Tetrahydrocannabinol (THC) sind in Tabelle 9 als Mittelwerte und Interquartilabstände genannt. Es sind die Parameter $t_{\max }, \mathrm{C}_{\max }, \mathrm{AUC}_{0-\infty}, \mathrm{Cl} / \mathrm{F}, \mathrm{t}_{1 / 2}$ terminal und $\mathrm{C}_{24 \mathrm{~h}}$ in den verschiedenen CYP2C9-GenotypGruppen dargestellt. Da hier keine zufällige Bevölkerungsstichprobe untersucht wurde, erfolgte keine gemeinsame statistische Analyse aller 43 Probanden.

Tab. 9 Pharmakokinetische Parameter von THC in Abhängigkeit vom CYP2C9-Genpolymorphismus

\begin{tabular}{|c|c|c|c|c|c|c|c|c|}
\hline \multirow[t]{2}{*}{ Parameter } & \multirow[t]{2}{*}{ Einheit } & \multicolumn{6}{|c|}{ CYP2C9 } & \multirow[t]{2}{*}{$P$} \\
\hline & & ${ }^{*} 1 /{ }^{*} 1$ & ${ }^{*} 1 / * 2$ & $*^{*} / * 2$ & $*^{*} / * 3$ & $* 2 / * 3$ & $* 3 / * 3$ & \\
\hline Anzahl & & 19 & 6 & 4 & 9 & 1 & 4 & \\
\hline \multirow[t]{2}{*}{$t_{\max }$} & $\mathrm{h}$ & 3.3 & 3.4 & 3.6 & 3.4 & 2.0 & 3.4 & n.s. \\
\hline & & $2.0-3.5$ & $2.0-5.0$ & $2.0-5.0$ & $2.7-5.0$ & & $2.3-3.6$ & \\
\hline \multirow[t]{2}{*}{$\mathrm{C}_{\max }$} & $\mu g / L$ & 2.7 & 2.7 & 2.6 & 3.0 & 4.4 & 6.3 & 0.05 \\
\hline & & $1.1-3.9$ & $1.6-3.6$ & $1.8-4.1$ & $2.3-4.6$ & & $2.0-9.7$ & \\
\hline \multirow[t]{2}{*}{$\mathrm{AUC}_{0-\propto}$} & $\mu g \cdot h / L$ & 16.1 & 20.2 & 16.2 & 31.7 & 19.8 & 49.7 & 0.001 \\
\hline & & $11.2-23.1$ & $13.3-24.4$ & $10.6-24.5$ & $20.3-38.2$ & & $22.5-72.5$ & \\
\hline \multirow[t]{2}{*}{$\mathrm{Cl} / \mathrm{F}$} & $\mathrm{L} / \mathrm{h}$ & 932 & 745 & 928 & 473 & 759 & 365 & 0.001 \\
\hline & & $648-1342$ & $614-1216$ & $639-1496$ & $395-740$ & & $207-682$ & \\
\hline \multirow[t]{2}{*}{$t_{1 / 2 \text { terminal }}$} & $\mathrm{h}$ & 7.5 & 7.3 & 7.2 & 15.1 & 8.6 & 22.1 & 0.09 \\
\hline & & $5.4-15.2$ & $5.1-11.3$ & $1.9-10.0$ & $6.6-18.7$ & & $16.7-33.3$ & \\
\hline \multirow[t]{2}{*}{$\mathrm{C}_{24 \mathrm{~h}}$} & $\mu g / L$ & 0.15 & 0.17 & 0.21 & 0.18 & 0.14 & 0.37 & 0.02 \\
\hline & & $0.12-0.17$ & $0.14-0.29$ & $0.20-0.21$ & $0.14-0.25$ & & $0.31-0.49$ & \\
\hline
\end{tabular}

Für alle Daten sind die Mediane und die Interquartilabstände angegeben. $t_{\max }$, Zeitpunkt der maximalen Plasmakonzentration; $\mathrm{C}_{\max }$, maximale Blutkonzentration; $\mathrm{AUC}_{0-\infty}$, Fläche unter der Konzentrations-Zeit-Kurve; Cl/F, Clearance dividiert durch Bioverfügbarkeit; $t_{1 / 2}$ terminal, terminale Halbwertszeit; $\mathrm{C}_{24 \mathrm{~h}}$, Plasmakonzentration 24 Stunden nach Medikamentenapplikation; $p$-Werte der Unterschiede in Abhängigkeit der CYP2C9-Genotypen (Jonckheere-Terpstra-Trend-Test); n.s., nicht signifikant ( $p$-Werte zwischen 0.05 und 0.1 sind angegeben, da sie mögliche Trends anzeigen können)

Die Zeitpunkte der maximalen THC-Plasmakonzentrationen $\left(t_{\max }\right)$ lagen zwischen 2 und 5 Stunden nach Medikamentengabe. Es traten hierbei keine statistisch signifikanten CYP2C9-Genotyp-basierten Unterschiede auf. Die maximalen THC-Plasmaspiegel reichten von $0.52 \mu \mathrm{g} / \mathrm{L}$ bis hin $\mathrm{zu} 10.1 \mu \mathrm{g} / \mathrm{L}$. Unter den CYP2C9-Genotypen fanden sich signifikante Divergenzen bei einem Median von 2.7 
$\mu \mathrm{g} / \mathrm{L}$ in Trägern der Variante CYP2C ${ }^{*} 1 /{ }^{*} 1$ im Vergleich zu einem Median von $6.3 \mu \mathrm{g} / \mathrm{L}$ bei der Allelkombination CYP2C $9 * 3 /{ }^{*} 3(p=0.05$, Jonckheere-TerpstraTrend-Test).

Die orale Clearance (Cl/F) bewegte sich zwischen 200 und $4000 \mathrm{~L} / \mathrm{h}$. Es ergab sich ein signifikanter Unterschied dieses Parameters hinsichtlich der Anzahl der CYP2C9*3-Allele. Die Mediane der vordefinierten Gruppen CYP2C9*1/*1, CYP2C9*1/*3 sowie CYP2C9*3/*3 lagen bei $932,473,365 \mathrm{~L} / \mathrm{h}(p=0.001)$. Bezüglich der oralen Clearance unterschieden sich die Träger des CYP2C9*1-Allels nicht von denen des CYP2C9*2-Allels. Die mediane terminale Halbwertszeit des THC betrug bei Trägern mit keinem, einem oder zwei CYP2C9*3-Allelen 7.5, 15.1 sowie $22.1 \mathrm{~h}$. Bei der THC-Plasmakonzentration zum Zeitpunkt 24 Stunden nach Medikamentengabe wurde für $37 \mathrm{der} 43$ Proben ein Wert oberhalb von $0.1 \mu \mathrm{g} / \mathrm{L}$ gemessen. Im Vergleich zu dem Median von $0.15 \mu \mathrm{g} / \mathrm{L}$ für CYP2C9*1/*1 war sie bei CYP2C9*3/*3-Trägern mit einem Median von $0.37 \mu \mathrm{g} / \mathrm{L}$ mehr als doppelt so hoch. In Abbildung 6 sind die THC-Plasmakonzentrationen aller Probanden in Abhängigkeit von der Anzahl der CYP2C9*3-Allele dargestellt. Zu erkennen ist auch an dieser Stelle wieder die interindividuelle Streuung unter den Probanden.

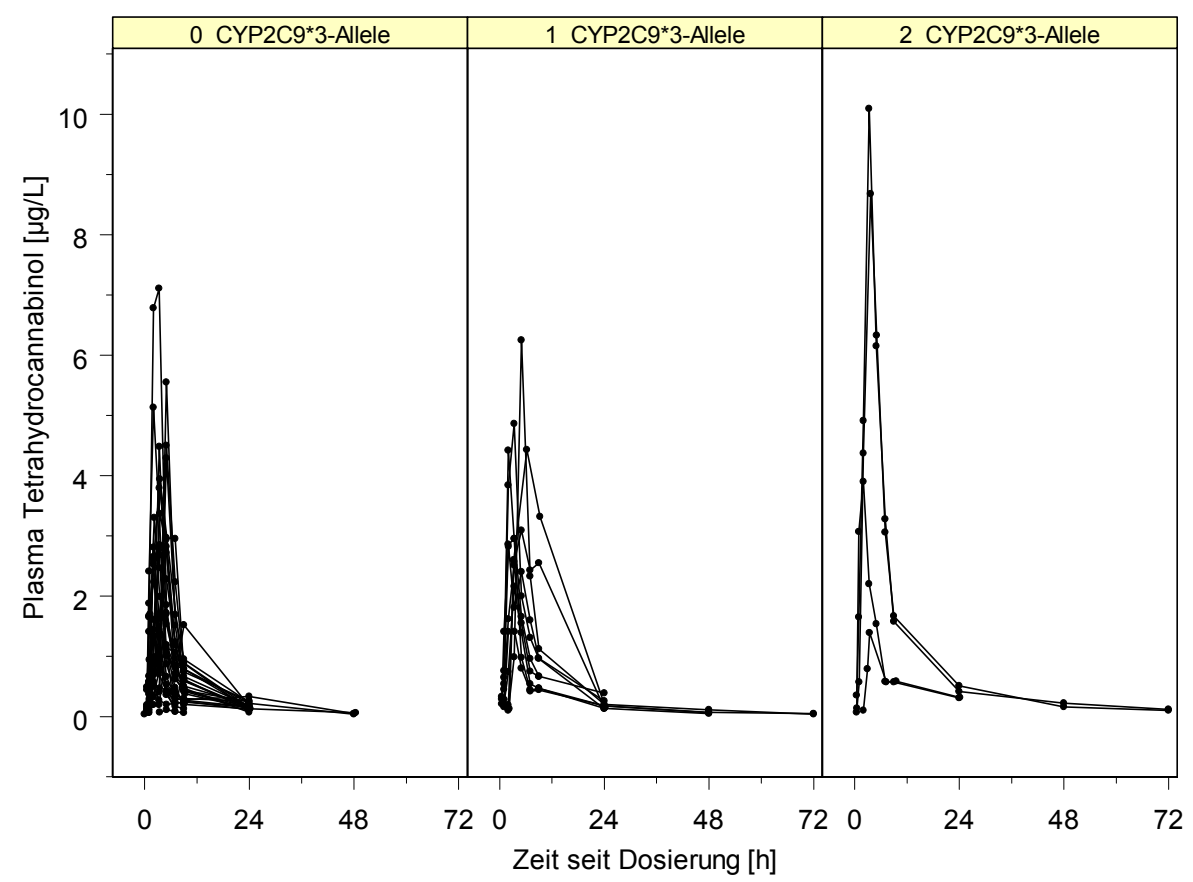

Abb. 6 THC-Plasmakonzentrationsverläufe aller Probanden in Abhängigkeit der Anzahl der CYP2C9*3-Allele. Der Einfluss des Genotyps ist hier nicht so klar zu erkennen wie in Tabelle 9. Dennoch wurde die Abbildung gezeigt, da sie die Variation auch innerhalb der Genotyp-Gruppen gut widerspiegelt. 
Die Mittelwerte und Standardabweichungen der Konzentrationen von THC im Blutplasma in den drei, nach der Anzahl der CYP2C9*3-Allele differenzierten Gruppen, sind in Abbildung 7 gezeigt. Auch in dieser Darstellung ist zu erkennen, dass die Plasmakonzentration von THC umso höher war, je mehr CYP2C9*3-Allele ein Proband trug.

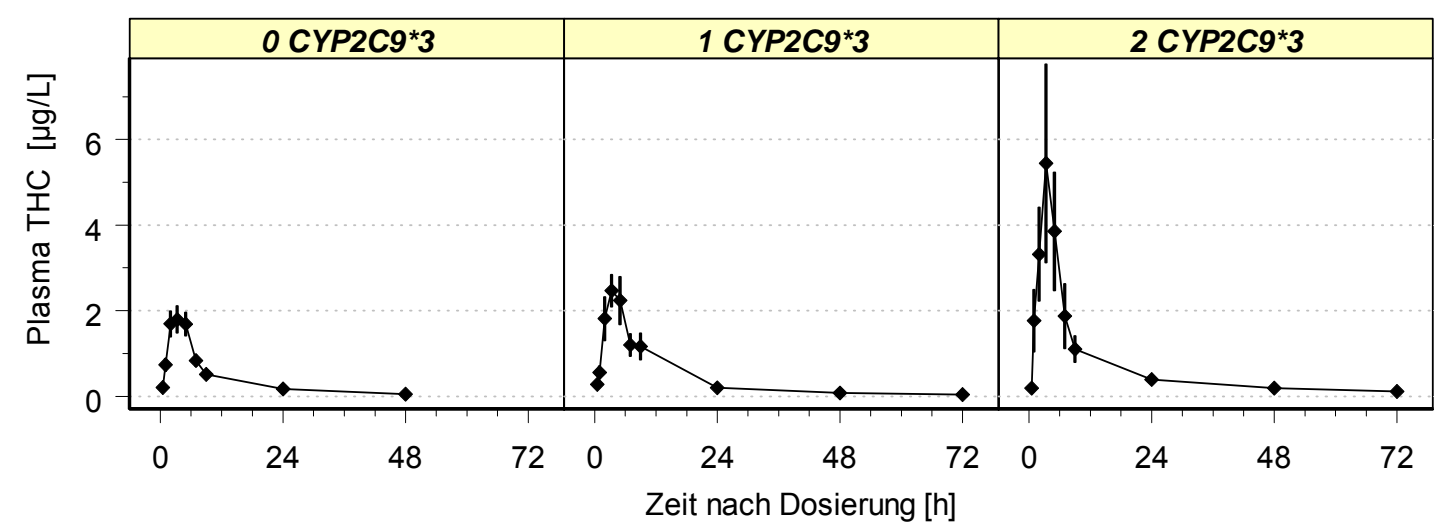

Abb. 7 Mittelwerte und Standardabweichungen der Konzentrationen von THC im Blutplasma in Abhängigkeit der Anzahl von CYP2C9*3-Allelen

Im Hinblick auf das Allel CYP2C9*2 fanden sich keine signifikanten Unterschiede für THC. Graphisch veranschaulicht wird dies in Abbildung 8 anhand von Boxplots zur Fläche unter der Konzentrations-Zeit-Kurve (AUC).

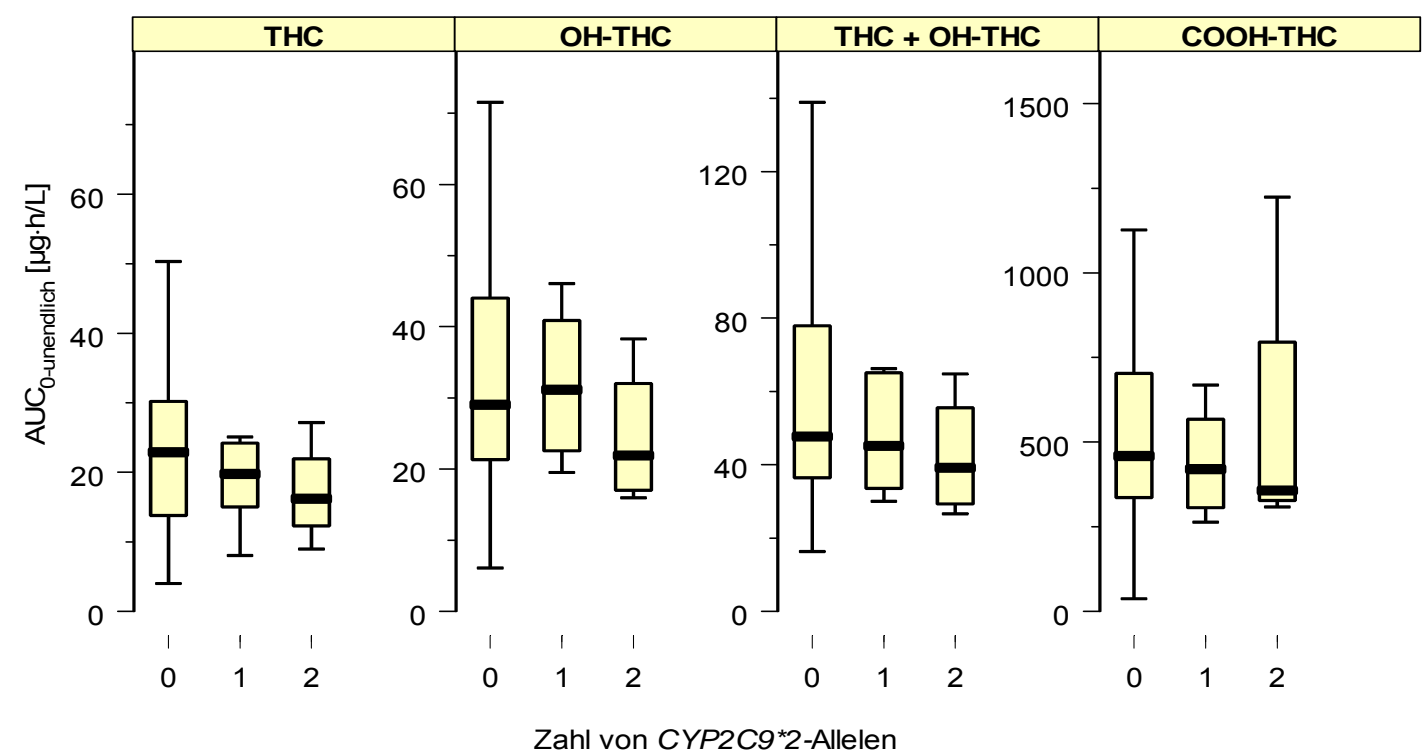

Abb. 8 Fläche unter der Konzentrations-Zeit-Kurve (AUC) von THC und seinen Metaboliten in Abhängigkeit der Zahl an CYP2C9*2-Allelen. Wie man sieht, war für das Allel *2 kein deutlicher Effekt zu erkennen. Am ehesten deutet sich ein Abfall der AUC mit zunehmender Zahl der CYP2C9*2-Allele an. Dies könnte ein schwacher Hinweis für eine eventuell gesteigerte Clearance bei *2-Trägern sein. 


\subsubsection{Pharmakokinetische Parameter von OH-THC}

Der primäre Metabolit, zu dem das THC verstoffwechselt wird, ist das 11-Hydroxy-Delta-9-Tetrahydrocannabinol (OH-THC). Ähnlich wie für das THC in Kapitel 3.2.2 sind nun für das $\mathrm{OH}-\mathrm{THC}$ in Tabelle 10 die Mittelwerte und die entsprechenden Interquartilabstände der Parameter $t_{\max }, C_{\max }, A U C_{0-\propto}$ und $C_{24 h}$ in Abhängigkeit der verschiedenen CYP2C9-Genotypen dargestellt.

Tab. 10 Pharmakokinetische Parameter von $\mathrm{OH}-\mathrm{THC}$ in Abhängigkeit vom CYP2C9Genpolymorphismus

\begin{tabular}{|c|c|c|c|c|c|c|c|c|}
\hline \multirow[t]{2}{*}{ Parameter } & \multirow[t]{2}{*}{ Einheit } & \multicolumn{6}{|c|}{ CYP2C9 } & \multirow[t]{2}{*}{$P$} \\
\hline & & ${ }^{*} 1 /{ }^{*} 1$ & ${ }^{*} 1 /{ }^{*} 2$ & ${ }^{*} 2 / * 2$ & ${ }^{*} 1 / * 3$ & $* 2 / * 3$ & *3/*3 & \\
\hline Anzahl & & 19 & 6 & 4 & 9 & 1 & 4 & \\
\hline$t_{\max }$ & $\mathrm{h}$ & $\begin{array}{c}3.3 \\
2.2-3.5\end{array}$ & $\begin{array}{c}3.4 \\
3.0-5.5\end{array}$ & $\begin{array}{c}4.2 \\
2.4-5.0\end{array}$ & $\begin{array}{c}3.4 \\
3.3-5.0\end{array}$ & 3.3 & $\begin{array}{c}3.4 \\
2.3-3.6\end{array}$ & n.s. \\
\hline $\mathrm{C}_{\max }$ & $\mu g / L$ & $\begin{array}{c}2.8 \\
2.0-5.8\end{array}$ & $\begin{array}{c}4.1 \\
2.8-4.5\end{array}$ & $\begin{array}{c}2.5 \\
1.6-2.9\end{array}$ & $\begin{array}{c}2.8 \\
2.4-4.0\end{array}$ & 6.5 & $\begin{array}{c}2.8 \\
1.1-5.1\end{array}$ & n.s. \\
\hline$A \cup C_{0-\infty}$ & $\mu g \cdot h / L$ & $\begin{array}{c}24.0 \\
20.7-43.7\end{array}$ & $\begin{array}{c}32.1 \\
21.8-42.2\end{array}$ & $\begin{array}{c}21.9 \\
16.5-35.2\end{array}$ & $\begin{array}{c}33.1 \\
25.0-46.2\end{array}$ & 31.1 & $\begin{array}{c}32.1 \\
15.9-65.7\end{array}$ & n.s. \\
\hline $\mathrm{C}_{24 \mathrm{~h}}$ & $\mu g / L$ & $\begin{array}{c}0.24 \\
0.16-0.41\end{array}$ & $\begin{array}{c}0.23 \\
0.08-0.36\end{array}$ & $\begin{array}{c}0.20 \\
0.16-0.34\end{array}$ & $\begin{array}{c}0.29 \\
0.19-0.47\end{array}$ & 0.04 & $\begin{array}{c}0.28 \\
0.14-0.70\end{array}$ & n.s. \\
\hline
\end{tabular}

Für alle Daten sind die Mediane und die Interquartilabstände angegeben. $t_{\max }$, Zeitpunkt der maximalen Plasmakonzentration; $\mathrm{C}_{\max }$, maximale Blutkonzentration; $\mathrm{AUC}_{0-\infty}$, Fläche unter der Konzentrations-Zeit-Kurve; $\mathrm{C}_{24 \mathrm{~h}}$, Plasmakonzentration 24 Stunden nach Medikamentenapplikation; p-Werte der Unterschiede in Abhängigkeit der CYP2C9-Genotypen (Jonckheere-Terpstra-TrendTest); n.s., nicht signifikant

Keiner der pharmakokinetischen Parameter wies in Abhängigkeit der CYP2C9-Genotypen Unterschiede mit statistischer Signifikanz auf. In den Abbildungen 9 und 10 sind die $\mathrm{OH}-\mathrm{THC}$-Plasmakonzentrationen und die mittleren Verläufe aller Probanden in Abhängigkeit von der Anzahl der CYP2C9*3-Allele dargestellt.

Bezogen auf das Allel CYP2C9*2 fanden sich keine signifikanten Unterschiede für $\mathrm{OH}$-THC (Abbildung 8). 


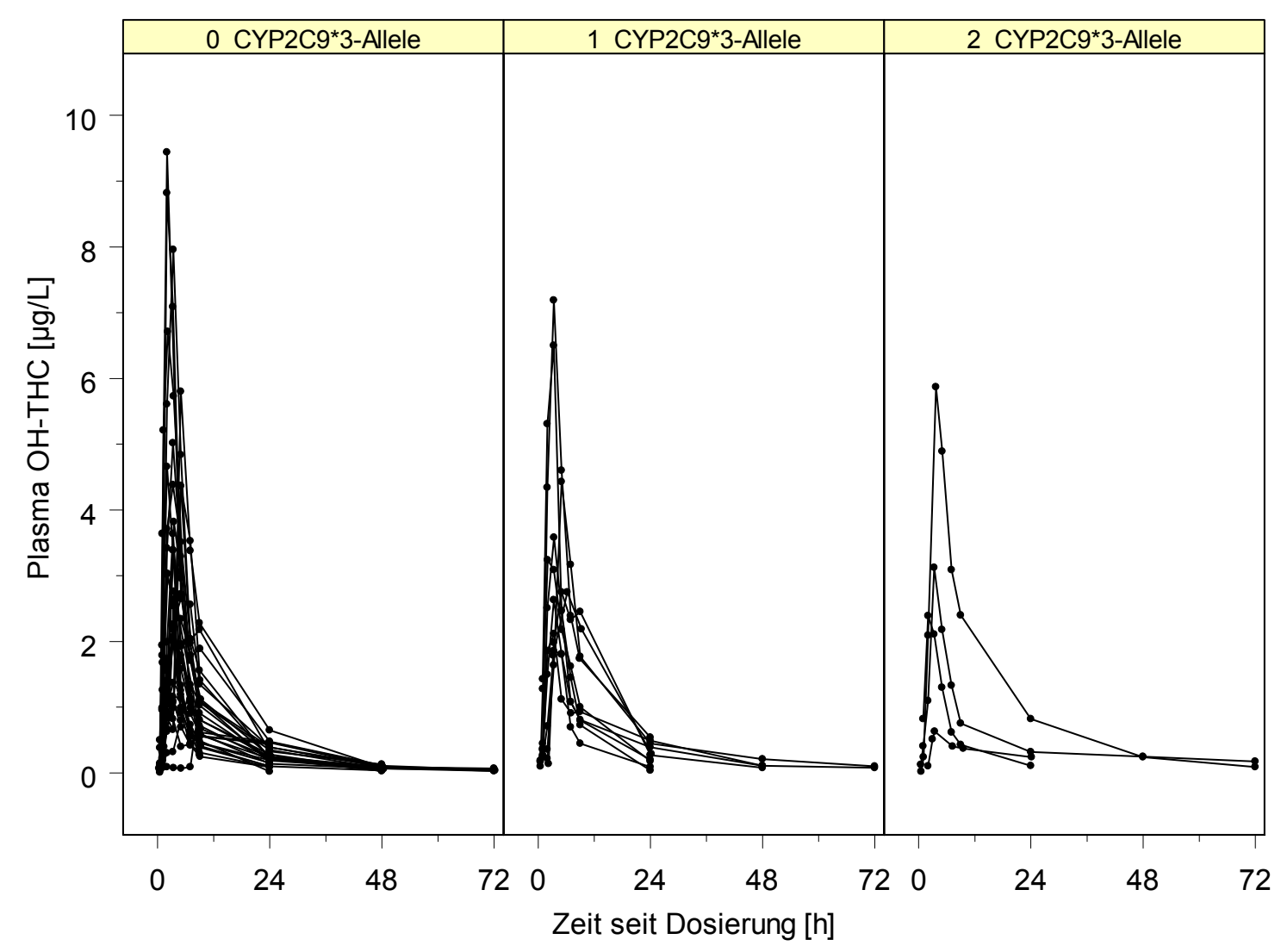

Abb. $9 \mathrm{OH}-\mathrm{THC}$-Plasmakonzentrationen aller Probanden in Abhängigkeit von der Anzahl der CYP2C9*3-Allele

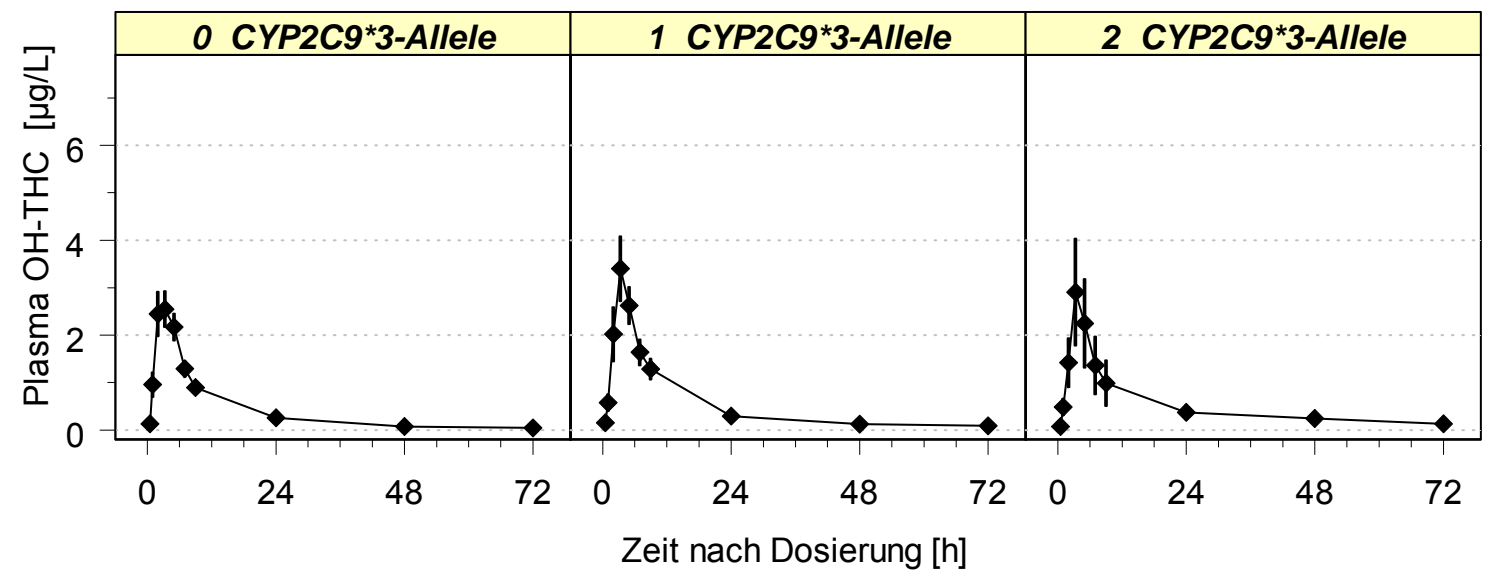

Abb. 10 Mittelwerte und Standardabweichungen der Konzentrationen von OH-THC im Blutplasma in Abhängigkeit der Zahl an CYP2C9*3-Allelen 


\subsubsection{Pharmakokinetische Parameter von $\mathrm{COOH}-\mathrm{THC}$}

Wie in den zwei vorangegangenen Kapiteln für THC und OH-THC sollen nun die pharmakokinetischen Parameter für den THC-Metaboliten 11-Nor-9-Carboxy-Delta9-Tetrahydrocannabinol ( $\mathrm{COOH}-\mathrm{THC})$ dargestellt werden. Tabelle 11 zeigt die Mittelwerte sowie die entsprechenden Interquartilabstände der Parameter $t_{\max }, \mathrm{C}_{\max }$, $\mathrm{AUC}_{0-\infty}$, und $\mathrm{C}_{24 \mathrm{~h}}$ in Abhängigkeit der verschiedenen CYP2C9-Genotypen.

Tab. 11 Pharmakokinetische Parameter von $\mathrm{COOH}-\mathrm{THC}$ in Abhängigkeit vom CYP2C9Genpolymorphismus

\begin{tabular}{|c|c|c|c|c|c|c|c|c|}
\hline \multirow[t]{2}{*}{ Parameter } & \multirow[t]{2}{*}{ Einheit } & \multicolumn{6}{|c|}{ CYP2C9 } & \multirow[t]{2}{*}{$P$} \\
\hline & & ${ }^{*} 1 /{ }^{*} 1$ & ${ }^{*} 1 /{ }^{*} 2$ & $* 2 / * 2$ & $* 1 / * 3$ & $* 2 / * 3$ & $* 3 / * 3$ & \\
\hline Anzahl & & 19 & 6 & 4 & 9 & 1 & 4 & \\
\hline$t_{\max }$ & $\mathrm{h}$ & $\begin{array}{c}3.4 \\
3.3-3.5\end{array}$ & $\begin{array}{c}3.4 \\
3.0-9.8\end{array}$ & $\begin{array}{c}4.2 \\
2.5-6.5\end{array}$ & $\begin{array}{c}3.4 \\
3.3-7.0\end{array}$ & 3.3 & $\begin{array}{c}3.4 \\
2.3-4.6\end{array}$ & n.s. \\
\hline $\mathrm{C}_{\max }$ & $\mu g / L$ & $\begin{array}{c}25.7 \\
18.0-41.3\end{array}$ & $\begin{array}{c}28.4 \\
19.8-35.8\end{array}$ & $\begin{array}{c}20.9 \\
13.6-27.9\end{array}$ & $\begin{array}{c}15.0 \\
11.4-16.6\end{array}$ & 26.6 & $\begin{array}{c}4.8 \\
2.0-8.9\end{array}$ & $<0.001$ \\
\hline $\mathrm{AUC}_{0-x}$ & $\mu g \cdot h / L$ & $\begin{array}{c}667 \\
396-1081\end{array}$ & $\begin{array}{c}478 \\
385-592\end{array}$ & $\begin{array}{c}357 \\
318-1010\end{array}$ & $\begin{array}{c}419 \\
330-537\end{array}$ & 263 & $\begin{array}{c}207 \\
52-328\end{array}$ & $<0.001$ \\
\hline $\mathrm{C}_{24 \mathrm{~h}}$ & $\mu g / L$ & $\begin{array}{c}8.6 \\
5.6-14.9\end{array}$ & $\begin{array}{c}7.1 \\
5.9-9.4\end{array}$ & $\begin{array}{c}5.4 \\
4.9-15.2\end{array}$ & $\begin{array}{c}6.1 \\
4.5-8.1\end{array}$ & 3.2 & $\begin{array}{c}1.6 \\
1.0-3.5\end{array}$ & $<0.001$ \\
\hline
\end{tabular}

Für alle Daten sind die Mediane und die Interquartilabstände angegeben. $t_{\max }$, Zeitpunkt der maximalen Plasmakonzentration; $\mathrm{C}_{\max }$, maximale Blutkonzentration; $\mathrm{AUC}_{0-\infty}$, Fläche unter der Konzentrations-Zeit-Kurve; $\mathrm{C}_{24 \mathrm{~h}}$, Plasmakonzentration 24 Stunden nach Medikamentenapplikation; p-Werte der Unterschiede in Abhängigkeit der CYP2C9 Genotypen (Jonckheere-Terpstra-Trend-Test); n.s., nicht signifikant

Die Mediane der maximalen $\mathrm{COOH}-\mathrm{THC}$ Plasmakonzentrationen $\left(\mathrm{C}_{\max }\right)$ betrugen für die Genotypen CYP2C9*1/*1,CYP2C9*1/*3 und CYP2C9*3/*3 25.7, 15.0 sowie $4.8 \mu \mathrm{g} / \mathrm{L}$ ( $p<0.001$, Jonckheere-Terpstra-Trend-Test). Ähnlich wie die Mittelwerte für $\mathrm{C}_{\max }$ korrelierten auch die AUC und die Mittelwerte für $\mathrm{C}_{24 \mathrm{~h}}$ negativ mit der Anzahl der CYP2C9*3-Allele. Es ergaben sich in Bezug auf die Genotypen CYP2C9 ${ }^{\star} 1 /{ }^{*} 1$, CYP2C9*1/*3 und CYP2C9*3/*3 mittlere Werte von 667, 357 und $207 \mu \mathrm{g}^{*} \mathrm{~h} / \mathrm{L}$ hinsichtlich der AUC sowie von 8.6, 5.4 und $1.6 \mu \mathrm{g} / \mathrm{L}$ für $\mathrm{C}_{24 \mathrm{~h}}(\mathrm{p}<0.001)$. Die maximale Plasmakonzentration und die $\mathrm{AUC}$ von $\mathrm{COOH}-\mathrm{THC}$ waren also umso kleiner, je mehr CYP2C9*3-Allele die Probanden trugen. 
In den Abbildungen 11 und 12 sind die $\mathrm{COOH}-\mathrm{THC}$-Plasmakonzentrationen sowie die mittleren Verläufe aller Probanden in Abhängigkeit von der Anzahl der CYP2C9*3-Allele dargestellt. Beide Abbildungen veranschaulichen graphisch die bereits in Tabelle 11 belegten erheblichen Unterschiede in den Konzentrationen des $\mathrm{COOH}-\mathrm{THC}$ in Abhängigkeit von der Zahl an CYP2C9*3-Allelen. Es wurde umso weniger $\mathrm{COOH}-\mathrm{THC}$ gebildet, je mehr CYP2C9*3-Allele die Versuchspersonen trugen.

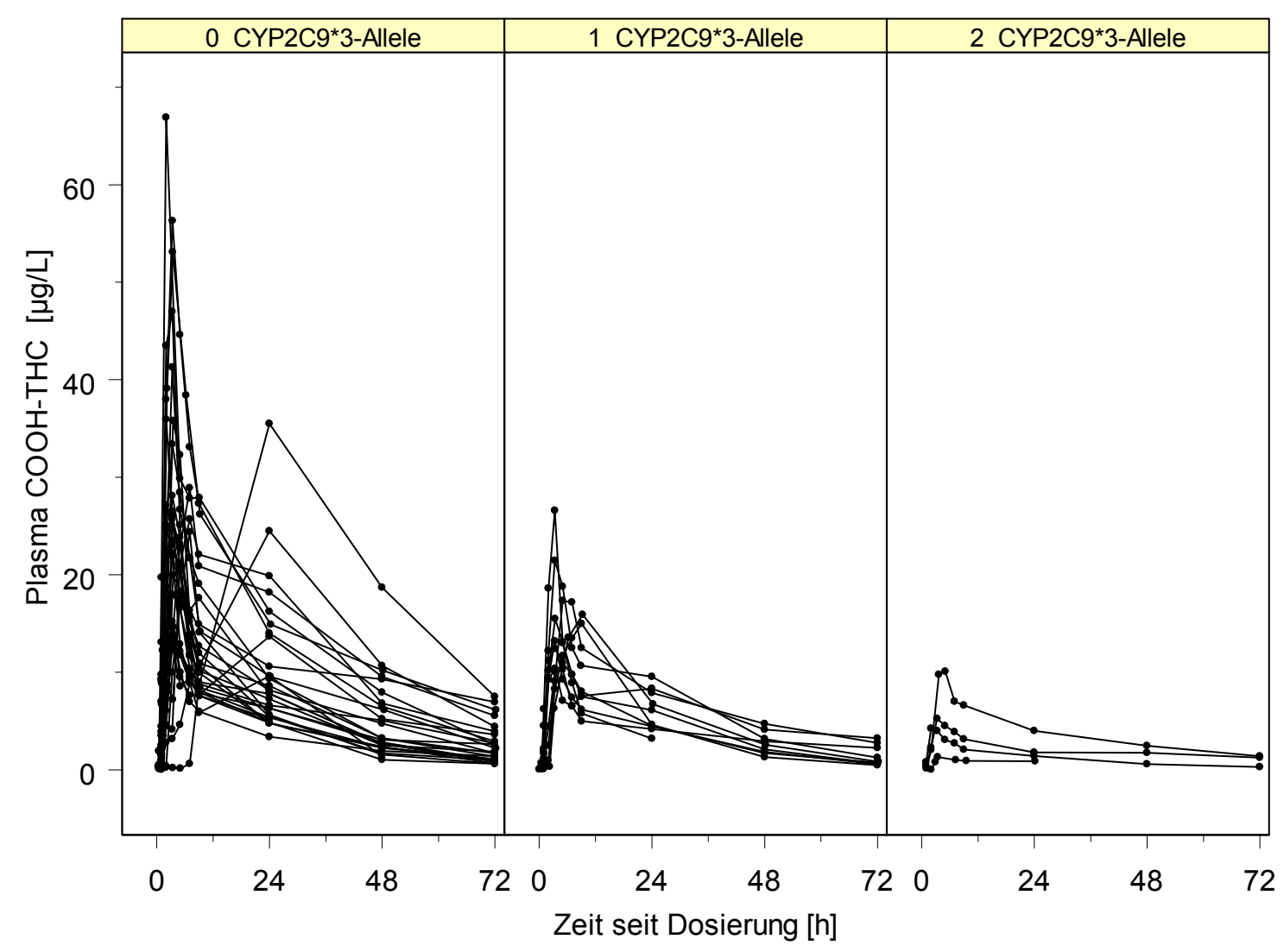

Abb. $11 \mathrm{COOH}-\mathrm{THC}$-Plasmakonzentrationen aller Probanden in Abhängigkeit von der Anzahl der CYP2C9*3-Allele 


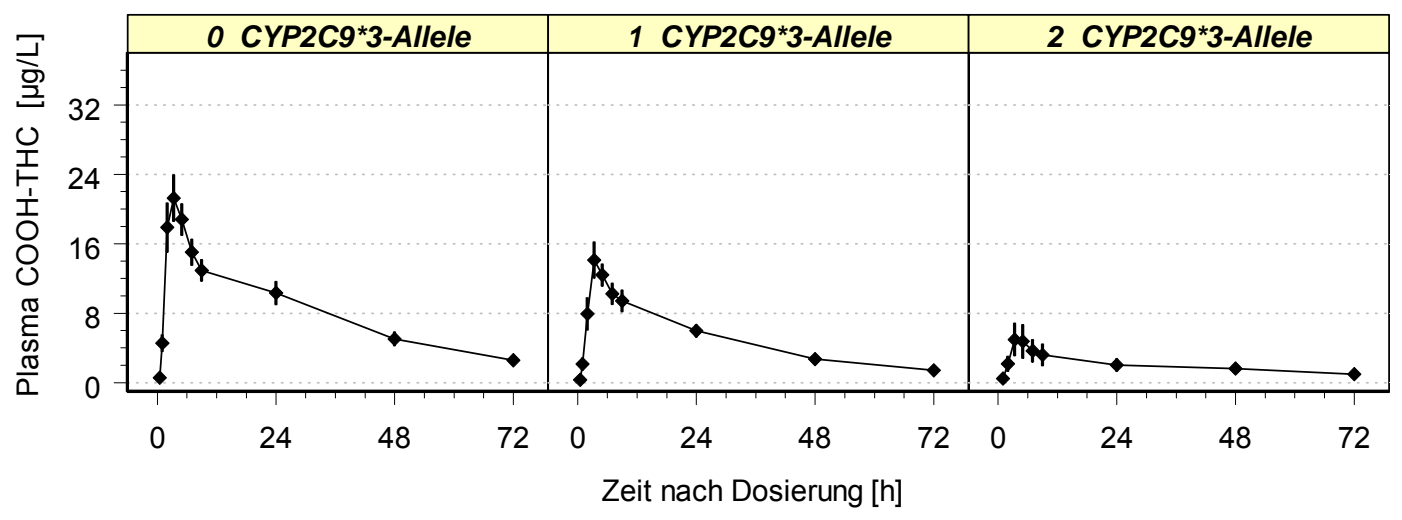

Abb. 12 Mittelwerte und Standardabweichungen der Konzentrationen von $\mathrm{COOH}-\mathrm{THC}$ im Blutplasma in Abhängigkeit der Anzahl von CYP2C9*3-Allelen

Wie in Abbildung 13 gezeigt, stieg der Quotient der medianen Plasmakonzentrationen aus $\mathrm{COOH}-\mathrm{THC}$ und THC 48 Stunden nach Medikamentengabe bei Trägern der Variante CYP2C ${ }^{*} 1 /{ }^{*} 1$ auf etwa 120 an, während er für den $\mathrm{CYP}_{2} \mathrm{C}^{*}{ }^{*} /{ }^{*} 3-\mathrm{G}$ erreichte. Genauer gesagt, betrugen die errechneten Quotienten für die Genotypen CYP2C9*1/*1, CYP2C9*1/*3 und CYP2C9*3/*3 123.3, 44.2 und 9.9. Dies könnte im Hinblick auf die forensische Medizin von Bedeutung sein, da der Quotient aus $\mathrm{COOH}-\mathrm{THC}$ und THC gelegentlich in der Bewertung des Cannabiskonsums eine Rolle spielt.

Bezüglich des Allels CYP2C9*2 ergaben sich keine signifikanten Unterschiede für $\mathrm{COOH}$-THC (Abbildung 8).

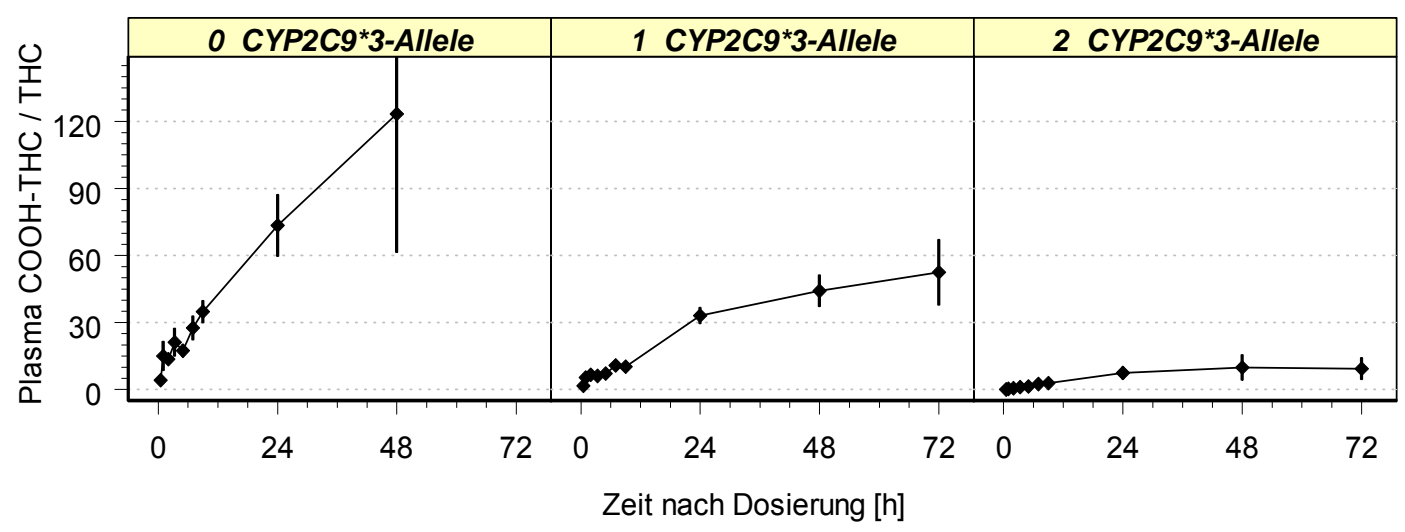

Abb. 13 Mittelwerte und Standardabweichungen der Quotienten aus $\mathrm{COOH}-\mathrm{THC}$ und THC im Blutplasma in Abhängigkeit der Zahl an CYP2C9*3-Allelen 


\subsection{Analyse der Pharmakokinetik von THC anhand von Kompartiment- Modellen}

Neben der so genannten nichtparametrischen Analyse der Pharmakokinetik von THC, OH-THC und $\mathrm{COOH}-\mathrm{THC}$ wurde auch eine pharmakokinetische KompartimentModellanalyse durchgeführt. Dabei wurde untersucht, mit welchen mathematischen Funktionen sich die Konzentrationsverläufe von THC am besten beschreiben lassen. Es ergab sich, dass die Pharmakokinetik von THC nicht durch ein Ein-KompartimentModell beschrieben werden kann (Abbildungen 4 und 5), da es initial zu einem sehr schnellen Konzentrationsabfall kam. Dagegen ließen sich die Einzelkurven jedoch gut durch ein Zwei-Kompartiment-Modell beschreiben. In Abbildung 14 sind die Plasma-THC-Konzentrations-Zeit-Kurven der einzelnen Probanden nach Messwerten (blau) und nach der berechneten Vorhersage der Modellanalyse (rot) dargestellt. Es ist zu erkennen, dass sich die individuellen Kurven durch die Modellanalyse gut beschreiben ließen. 


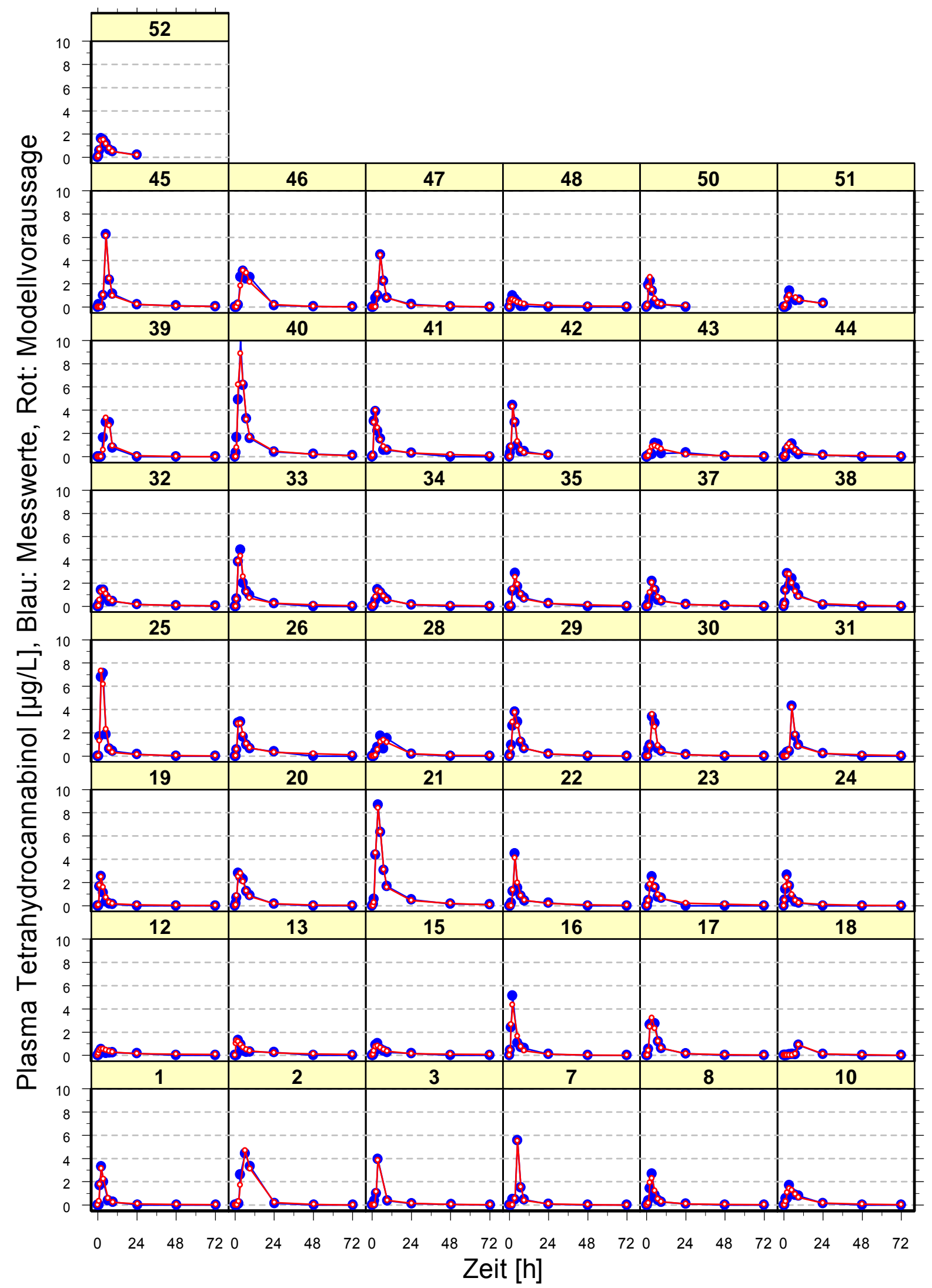

Abb. 14 Konzentrations-Zeit-Kurven der einzelnen Probanden nach Messwerten und Voraussage durch die Modellanalyse; Nummern von 1 bis 52 entsprechen den Probanden-Codes der Studienteilnehmer; insgesamt $n=43$ 
Aus der Modellanalyse ergaben sich für jeden Versuchsteilnehmer die individuellen pharmakokinetischen Kenngrößen. Es zeigte sich, dass die individuelle Absorption am besten durch eine mittlere Absorptionszeit (MAT, mean absorption time) und die Breite der mittleren Absorptionszeit (CV, coefficient of variation) beschrieben werden konnte, wobei letzterer Wert anders definiert ist als die gleich lautende statistische Kenngröße. Hier bedeutet CV anschaulich also die Breite der Zeit, in der die Substanz resorbiert wird (Ring et al. 2000). Mit Hilfe der Modellanalyse ließ sich ferner ein Verteilungsvolumen (V) bestimmen. Genau wie die Clearance ist auch das Verteilungsvolumen abhängig von der Bioverfügbarkeit und wurde somit als Quotient (V/F) angegeben. Des Weiteren erfolgte die Beschreibung des Zwei-KompartimentModells anhand der Geschwindigkeit, mit der die Substanz vom zentralen Kompartiment in das periphere übertritt (k23) und wieder zurücktransportiert wird (k32). Eine Veranschaulichung dieser Prozesse ist in Abbildung 15 dargestellt.

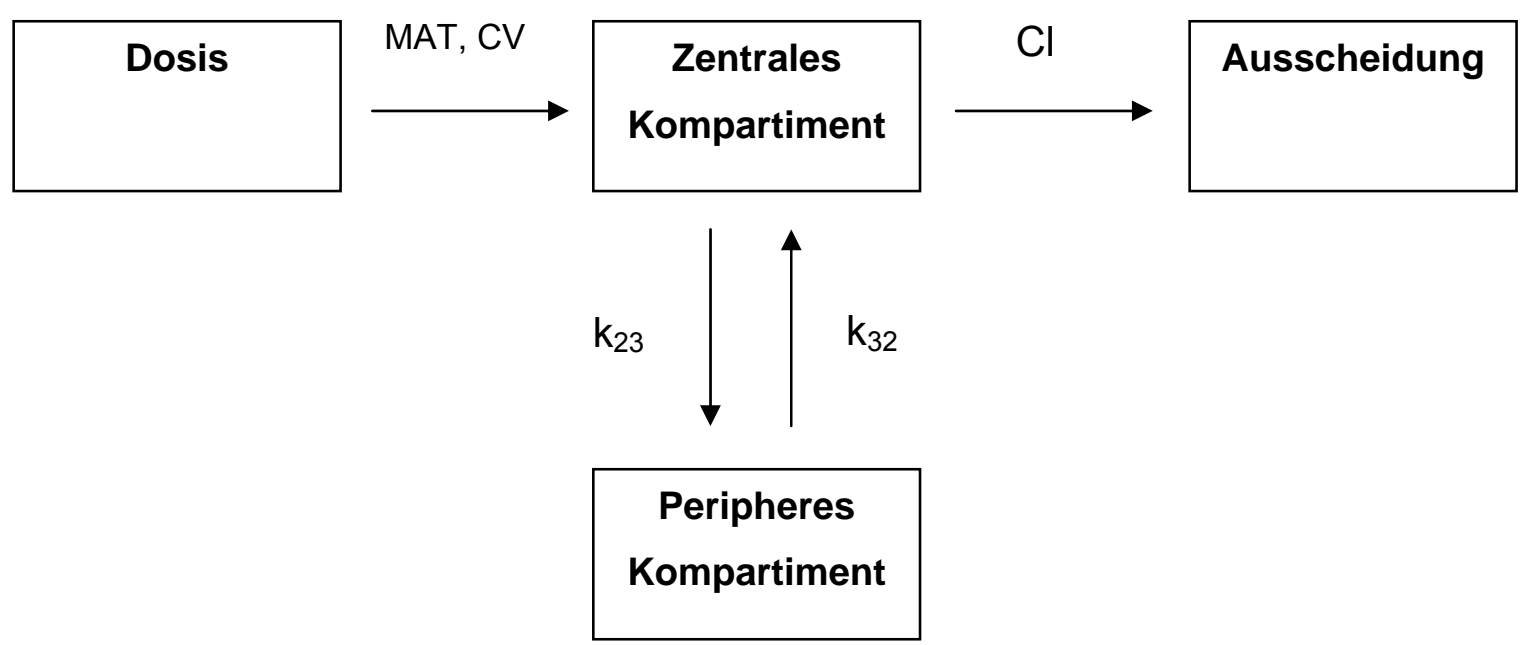

Abb. 15 Zwei-Kompartiment-Modell; MAT, mittlere Absorptionszeit; CV, Breite der mittleren Absorptionszeit; $\mathrm{Cl}$, Clearance; $\mathrm{k}_{23}$, Geschwindigkeit, mit der die Substanz vom zentralen Kompartiment in das periphere übertritt; $k_{32}$, Geschwindigkeit, mit der die Substanz wieder zurücktransportiert wird

Die Parameter der Modellanalyse sind in Tabelle 12 dargestellt. Die Bestimmung für die Clearance des individuellen Genotyps erfolgte differenziert nach der Zahl an CYP2C9*3-Allelen. So ergab sich je ein Mittelwert für die Gruppe mit 0 *3-Allelen $\left(C Y P 2 C 9{ }^{*} 1 /{ }^{*} 1,{ }^{*} 1 /{ }^{*} 2,{ }^{*} 2 /{ }^{*}\right), 1{ }^{*} 3$-Allel $\left(C Y P 2 C 9 * 1 /{ }^{*},{ }^{*} 2 /{ }^{*}\right)$ und $2{ }^{*} 3-$ Allelen (CYP2C9*3/*3). 
Tab. 12 Individuelle pharmakokinetische Parameter anhand der Modellanalyse

\begin{tabular}{|c|c|c|c|c|}
\hline Parameter & & Mittelwert & $\begin{array}{l}\text { Variations- } \\
\text { Koeffizient }\end{array}$ & 95\%-Vertrauensbereich* \\
\hline \multirow[t]{3}{*}{$\mathrm{Cl} / \mathrm{F}[\mathrm{L} / \mathrm{h}]$} & CYP2C ${ }^{*} 1 / * 1, * 1 / * 2, * 2 / * 2$ & 833 & 65.0 & $451-1540$ \\
\hline & CYP $2 C 9 * 1 / * 3, * 2 / * 3$ & 584 & 61.6 & $316-1078$ \\
\hline & $C Y P 2 C 9 * 3 / * 3$ & 334 & 87.1 & $181-617$ \\
\hline $\mathrm{V} / \mathrm{F}[\mathrm{L}]$ & & 2795 & 392 & $494-15802$ \\
\hline $\mathrm{K}_{23}[1 / \mathrm{h}]$ & & 0.227 & 0.032 & \\
\hline $\mathrm{K}_{32}[1 / \mathrm{h}]$ & & 0.058 & 0.006 & \\
\hline MAT [h] & & 2.67 & 0.241 & $1.04-6.87$ \\
\hline CV & & 0.392 & 0.052 & $0.15-1.04$ \\
\hline
\end{tabular}

$\mathrm{Cl} / \mathrm{F}$, Clearance dividiert durch Bioverfügbarkeit; V/F, Verteilungsvolumen dividiert durch Bioverfügbarkeit; k23; Geschwindigkeit, mit der die Substanz vom zentralen in das periphere Kompartiment übertritt; k32, Geschwindigkeit, mit der die Substanz vom peripheren in das zentrale Kompartiment übertritt; MAT, mittlere Absorptionszeit; CV, Breite der mittleren Absorptionszeit ${ }^{*}$ Mit dem speziellen, hier verwendeten Programm NONMEM (GloboMax LLC, Nonmem Project Team, Hanover, USA) war eine Unterscheidung der Präzision der Abschätzung der individuellen Parameter (Variationskoeffizient) von der Variation des Parameters in der Studienpopulation (95\%-Vertrauensbereich) möglich. Jedoch war es bei der begrenzten Stichprobenzahl von 43 Probanden nicht möglich, in jedem Fall die Variation in der Bevölkerung zu bestimmen.

\subsection{THC-Pharmakokinetik und CYP3A5-Genpolymorphismus}

Unter den Trägern des Genotyps CYP2C9*1/*1 fanden sich 13 Träger der inaktiven Variante CYP $3 A 5^{*} 3 /{ }^{*} 3$ sowie 6 Studienteilnehmer mit dem aktiven Genotyp CYP3A5*1/*3. In dieser Subgruppe von $\mathrm{n}=19$ Probanden ergaben sich weder für das THC selbst noch für seine Metaboliten $\mathrm{OH}-\mathrm{THC}$ und $\mathrm{COOH}-\mathrm{THC}$ CYP3A5-abhängige Unterschiede. Die mediane orale Clearance war für THC bei den 6 Trägern der aktiven Variante mit $941 \mathrm{~L} / \mathrm{h}$ marginal größer als bei den 13 Trägern der inaktiven Variante mit $933 \mathrm{~L} / \mathrm{h}$ (Abbildung 16) Für $\mathrm{OH}-\mathrm{THC}$ und $\mathrm{COOH}-\mathrm{THC}$ ergab sich ein ähnlicher Trend. Die Mediane der CYP3A5-aktiven Probanden lagen leicht über denen derer, die für CYP3A5 inaktiv waren. 


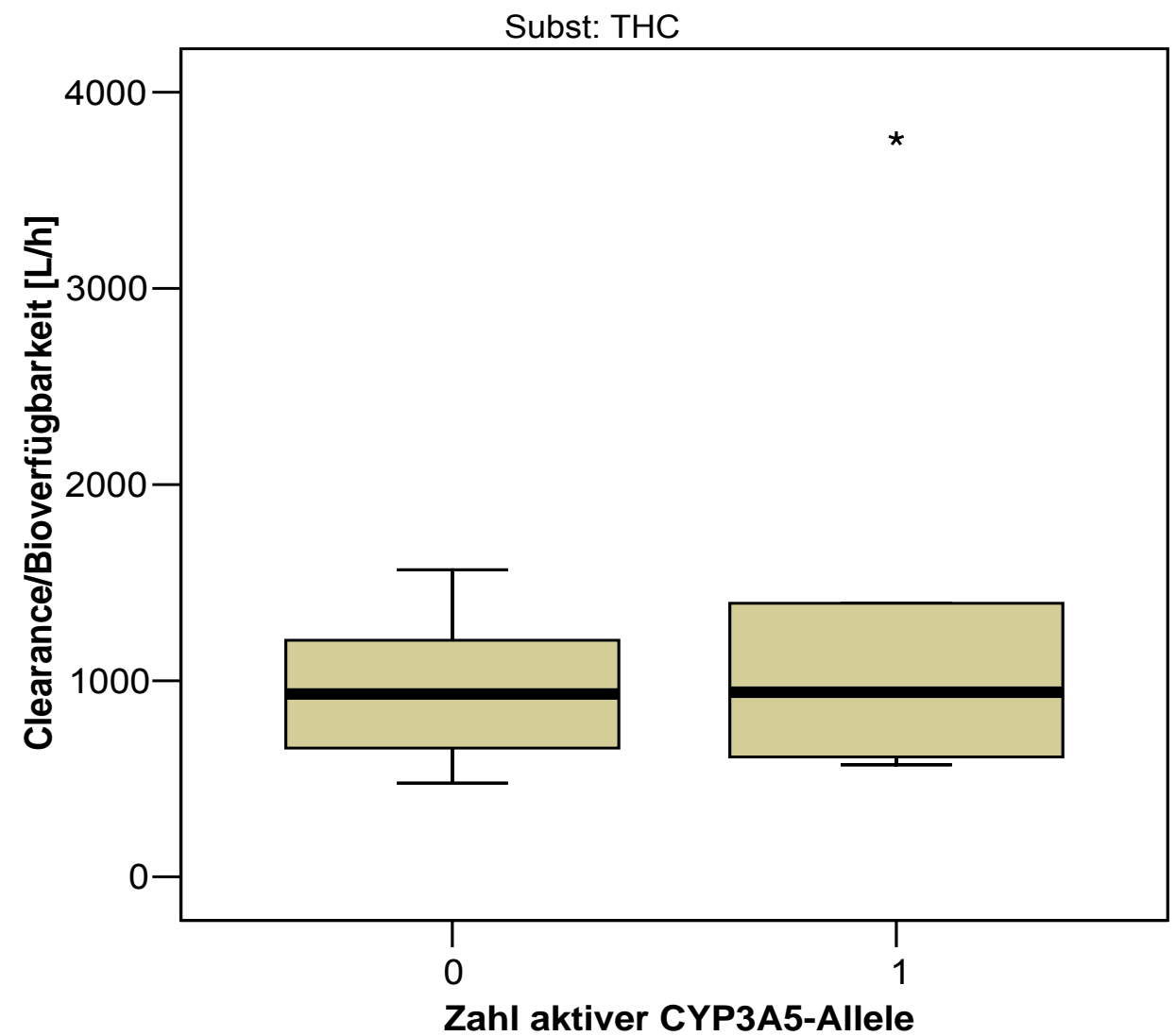

Abb. 16 Orale Clearance von THC in Abhängigkeit der Zahl an aktiven CYP3A5-Allelen (CYP3A5*1/*3). Daten von 13 Personen mit 2 inaktiven CYP3A5-Allelen und 6 Personen mit 1 aktiven CYP3A5-Allel.

Bezüglich der maximalen Blutkonzentrationen $\left(\mathrm{C}_{\max }\right)$ ergaben sich Mediane für THC, $\mathrm{OH}-\mathrm{THC}$ und auch für $\mathrm{COOH}-\mathrm{THC}$, die bei den 6 Probanden des Genotyps CYP $3 A 5 * 1 / * 3$ höher waren, als bei den Studienteilnehmern, die den Genotyp CYP $3 A 5^{\star} 3 /{ }^{*} 3$ trugen. Diese Ergebnisse waren jedoch statistisch nicht signifikant. 


\subsection{Pharmakokinetische Daten in Abhängigkeit vom OATP1B1- Genpolymorphismus}

Bezüglich des OATP1B1-Genpolymorphismus sollte untersucht werden, ob die Allele 130 Aspartat (Asn130Asp) und 174 Alanin (Val174Ala) die Transportaktivität von THC und seinen Metaboliten in die Hepatozyten der Leber verändern und damit einen Einfluss auf die Pharmakokinetik von Dronabinol ausüben.

Unter den Studienteilnehmern fanden sich 18 heterozygote und 7 homozygote Träger des $130_{\text {Aspartat }}$ Allels (Asn130Asp), während 17 Probanden heterozygote Träger und 2 Probanden homozygote Träger des $174_{\text {Alanin }}$-Allels (Val174Ala) waren. Obgleich geringfügige Effekte dieser OATP1B1-Genotypen angesichts der Fallzahlen nicht auszuschließen sind, ergaben sich in unseren Daten keine Hinweise auf Unterschiede bei der Pharmakokinetik von THC.

\subsection{Pharmakokinetik und Geschlecht}

Hinsichtlich des Geschlechts traten Unterschiede in der Pharmakokinetik von THC und seinen Metaboliten $\mathrm{OH}-\mathrm{THC}$ und $\mathrm{COOH}-\mathrm{THC}$ auf. Die Mediane der maximalen Blutkonzentrationen $\left(\mathrm{C}_{\max }\right)$ waren bei Frauen höher als bei Männern. Für das THC war dieser Unterschied statistisch signifikant ( $p=0.001$, Mann-Whitney-U-Test). Dies galt auch für die Summe der beiden psychoaktiven Substanzen THC und $\mathrm{OH}$-THC ( $p=0.001)$. Die zugehörigen Messwerte sind in den Abbildungen 17 und 18 illustriert. Im Hinblick auf $\mathrm{OH}-\mathrm{THC}$ alleine und $\mathrm{COOH}-\mathrm{THC}$ konnte nur ein entsprechender Trend zu höheren Konzentrationen bei Frauen festgestellt werden, jedoch keine statistische Signifikanz. Die Mediane für $\mathrm{OH}-\mathrm{THC}$ waren $2.6 \mu \mathrm{g} / \mathrm{L}$ bei den Männern und $4.1 \mu \mathrm{g} / \mathrm{L}$ bei den Frauen. Für $\mathrm{COOH}-\mathrm{THC}$ ergaben sich Mediane von $20.0 \mu \mathrm{g} / \mathrm{L}$ bei den Männern und $24.0 \mu \mathrm{g} / \mathrm{L}$ bei den Frauen. 


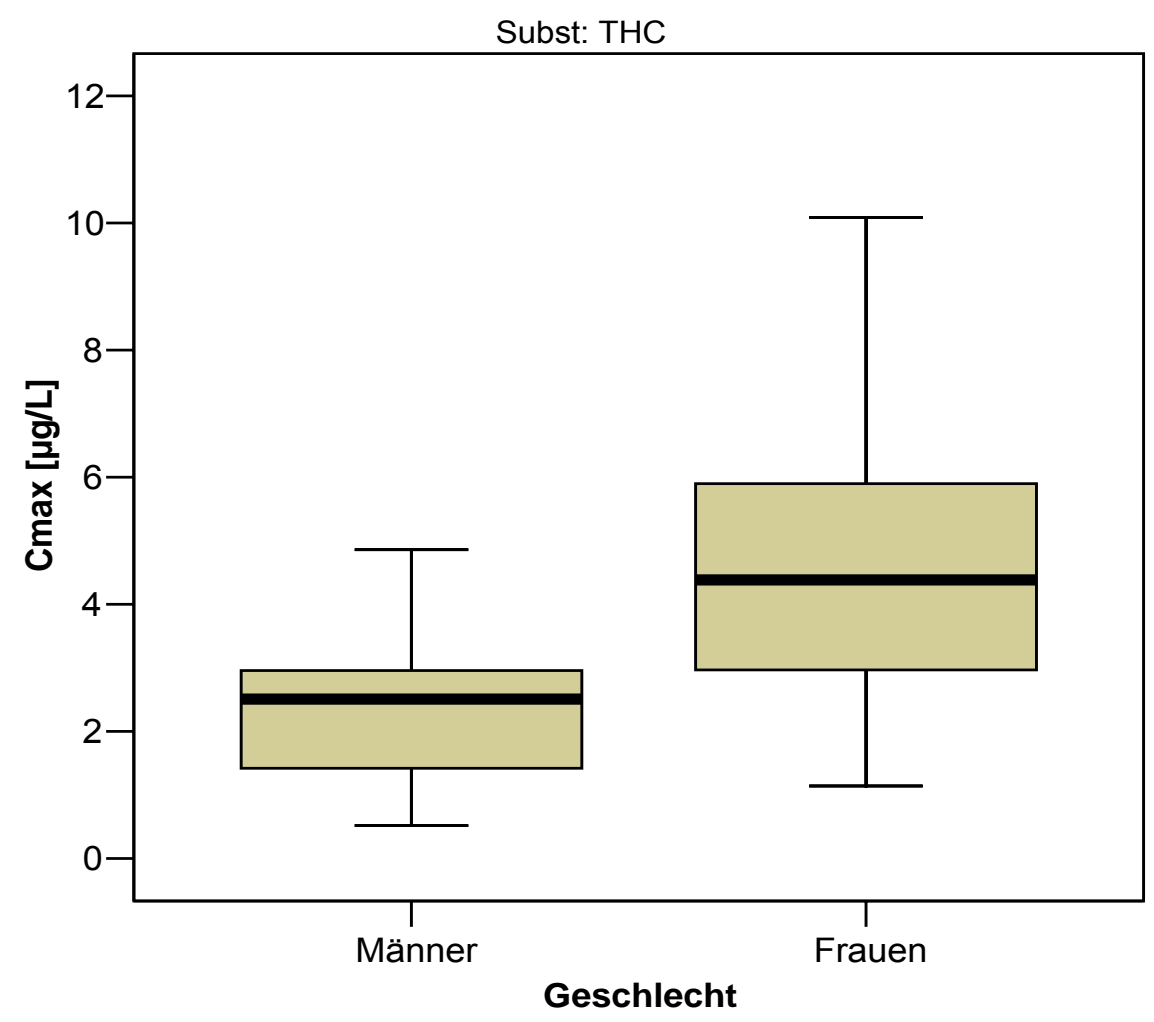

Abb. 17 Maximale Blutkonzentrationen von THC in Abhängigkeit des Geschlechts der Probanden

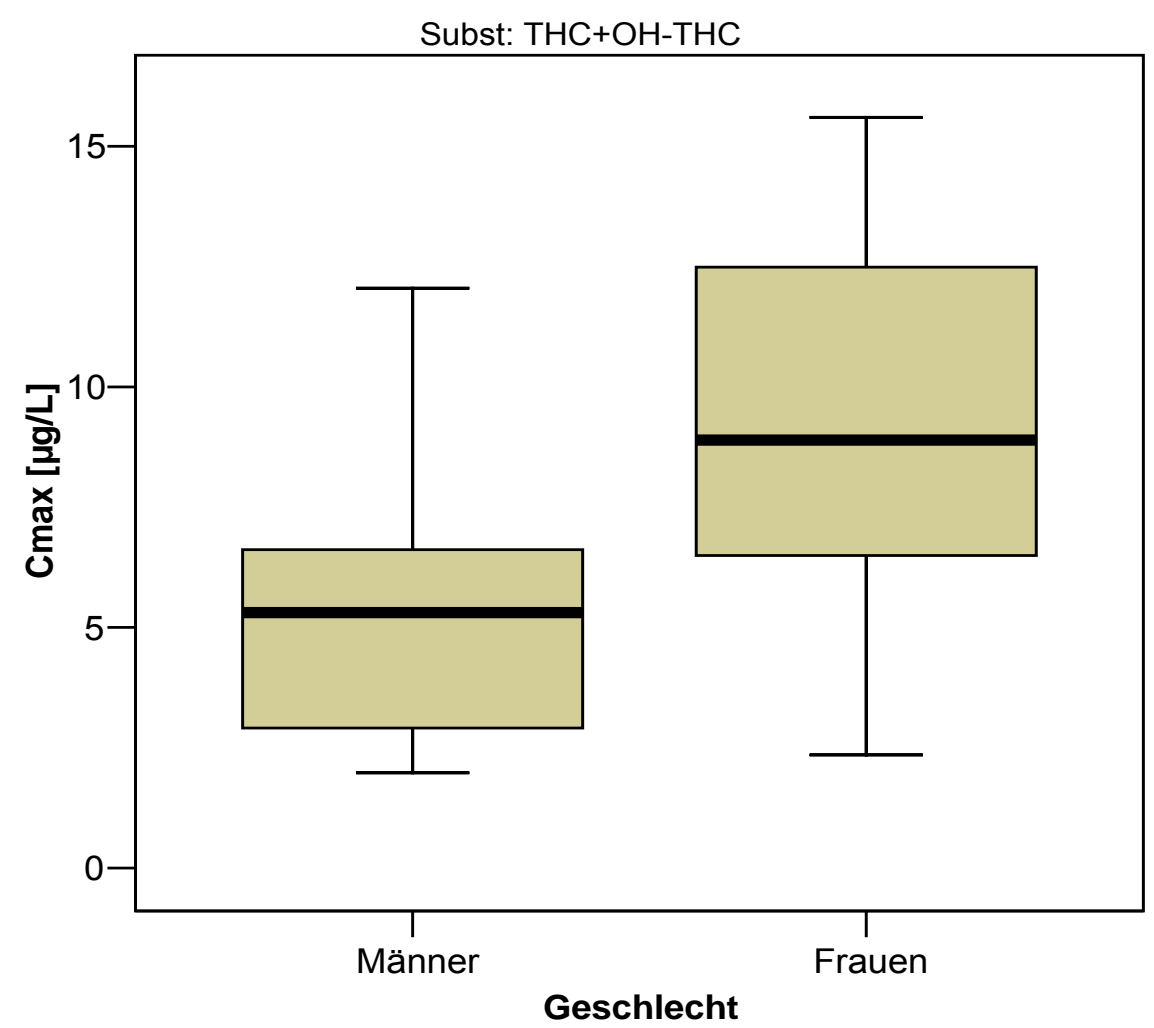

Abb. 18 Maximale Blutkonzentrationen in der Summe von THC und $\mathrm{OH}-\mathrm{THC}$ in Abhängigkeit des Geschlechts der Probanden 
Für die orale Clearance ( $\mathrm{Cl} / \mathrm{F})$ ergaben sich entsprechende Ergebnisse. Die Mediane waren bei Männern stets höher als bei den Frauen (für THC, $p=0.003$, Mann-Whitney-U-Test; für OH-THC, $\mathrm{p}<0.001)$. Hinsichtlich des THC-Metaboliten $\mathrm{COOH}-\mathrm{THC}$ ließ sich erneut nur ein entsprechender Trend zu höheren Werten bei den Männern erkennen. Die Mediane betrugen $35.8 \mathrm{~L} / \mathrm{h}$ bei Männern und $31.1 \mathrm{~L} / \mathrm{h}$ bei den Frauen.

Ein Effekt der CYP2C9*3-Allele auf die Pharmakokinetik von THC war in beiden Geschlechtern zu erkennen. Betrachtet man die maximalen Blutkonzentrationen $\left(\mathrm{C}_{\max }\right)$ für $\mathrm{THC}$ in Abhängigkeit der Zahl an CYP2C9*3-Allelen, so wurde deutlich, dass die maximalen Blutkonzentrationen der Frauen höher als die der männlichen Probanden waren, wenn sie 0 oder 2 CYP2C9*3-Allele trugen. Die Ergebnisse sind in Tabelle 13 zusammengestellt. Bei 2 CYP2C9*3-Allelen entsprachen die Werte für die Frauen, mit Ausnahme von $\mathrm{COOH}-\mathrm{THC}$, dem Dreifachen derer, die für die Männer gemessen wurden. Bei Trägern von 0 CYP2C9*3-Allelen verdoppelten sich die Messwerte bei den Frauen in etwa. Auch hier bildete COOH-THC eine Ausnahme. Die Messwerte der Frauen waren nur leicht erhöht. Bei den Studienteilnehmern, die nur ein CYP2C9*3-Allel trugen, waren die maximalen Blutkonzentrationen von Männern und Frauen annähernd gleich.

Tab. 13 Geschlechtsspezifische maximale Blutkonzentrationen $\left(C_{\max }\right)$ in Abhängigkeit der Zahl an CYP2C9*3-Allelen

\begin{tabular}{|c|c|c|c|c|c|c|c|}
\hline & \multirow{2}{*}{$\begin{array}{c}\text { Zahl an } \\
\text { CYP2C9*3- } \\
\text { Allelen }\end{array}$} & \multicolumn{2}{|c|}{0} & \multicolumn{2}{|c|}{1} & \multicolumn{2}{|c|}{2} \\
\hline & & Männer & Frauen & Männer & Frauen & Männer & Frauen \\
\hline \multirow{4}{*}{ 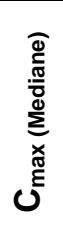 } & THC & 1.7 & 4.3 & 3.0 & 3.1 & 2.6 & 9.4 \\
\hline & $\mathrm{OH}-\mathrm{THC}$ & 2.3 & 4.4 & 3.2 & 2.8 & 1.5 & 4.5 \\
\hline & $\mathrm{COOH}-\mathrm{THC}$ & 23.9 & 28.9 & 15.5 & 15.0 & 2.8 & 7.7 \\
\hline & $\mathrm{THC}+\mathrm{OH}-\mathrm{THC}$ & 4.6 & 7.9 & 6.5 & 5.8 & 4.2 & 13.9 \\
\hline
\end{tabular}

Bei der Auftrennung nach der Zahl an CYP2C9*3-Allelen zeigte sich, dass die orale Clearance $(\mathrm{Cl} / \mathrm{F})$ bei Männern aller Allelkonstellationen größer war, als die der Frauen. Auch hier war der quantitativ bedeutendste Unterschied bei Trägern des CYP2C9*3/*3-Genotyps zu beobachten. Die Werte der Männer waren gegenüber denen der Frauen mehr als dreifach erhöht. Dies galt für THC (628 vs. 209 L/h), 
$\mathrm{OH}-\mathrm{THC}(938$ vs. $261 \mathrm{~L} / \mathrm{h}$ ), COOH-THC (281 vs. $46 \mathrm{~L} / \mathrm{h}$ ) sowie für die Summe aus THC und $\mathrm{OH}-\mathrm{THC}$ (377 vs. $122 \mathrm{~L} / \mathrm{h}$ ). Exemplarisch ist eine Illustration der Werte für THC in Abbildung 19 gezeigt.

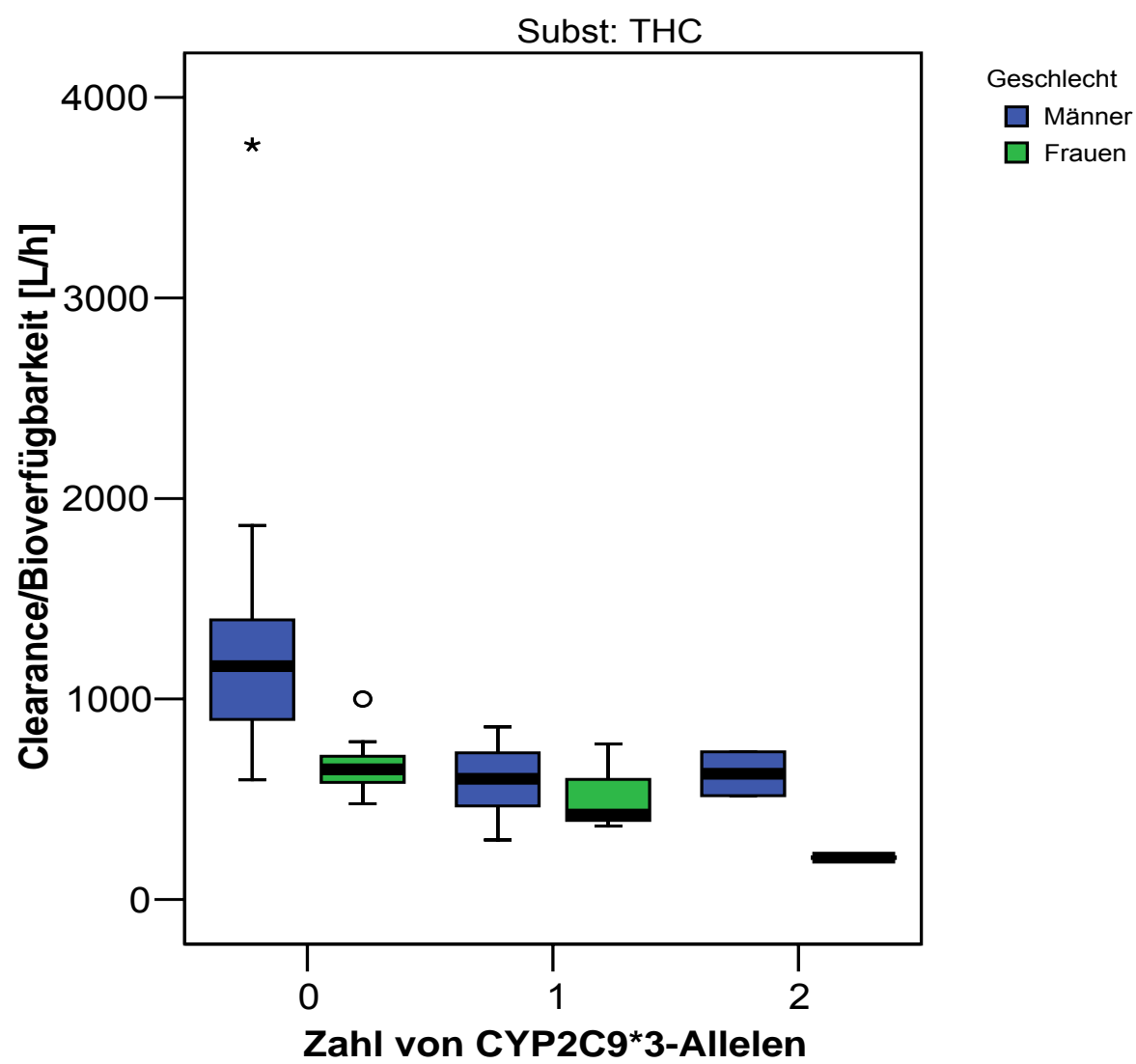

Abb. 19 Geschlechtsspezifische orale Clearance in Abhängigkeit der Zahl anCYP2C9*3-Allelen 


\subsection{Ausscheidung in den Urin}

Die Urinausscheidung von Medikamenten kann wichtige Hinweise zu den Metabolisierungs- und Eliminationswegen geben. Bei überwiegend renaler Ausscheidung ergeben sich aus den im Urin ausgeschiedenen Mengen auch Daten zur Bioverfügbarkeit. Um hier die Ausscheidungsprodukte des THC-Metabolismus im Urin bestimmen zu können, wurde der Urin der Probanden über 24 Stunden in fünf Fraktionen gesammelt. Die Sammelintervalle waren wie folgt gestaffelt: $0-3,3-6$, $6-9,9-12,12-24$ Stunden nach Medikamentengabe. Aus den Sammelurinen wurden Proben zur späteren Analyse entnommen und bei $-20^{\circ} \mathrm{C}$ tiefgefroren.

\subsubsection{Ausscheidung von $\mathrm{THC}-\mathrm{COOH}$ in den Urin}

$\mathrm{COOH}-\mathrm{THC}$ wird im Urin fast ausschließlich in Form des $\mathrm{COOH}-\mathrm{THC}$-Glukuronids ausgeschieden. Daher erfolgte bei der Analyse der Urine lediglich die quantitative Bestimmung des Carboxy-Metaboliten des THC, COOH-THC (Nachweisgrenze: $0,4 \mathrm{ng} / \mathrm{ml}$ ). Auf die Analyse des nicht glukuronidierten Metaboliten wurde verzichtet. Wenn im Folgenden die Rede von $\mathrm{COOH}-\mathrm{THC}$ im Urin ist, ist also stets daran zu denken, dass hier das Glukuronid gemeint ist (anders als im Plasma, wo tatsächlich das nicht-glukuronidierte $\mathrm{COOH}-\mathrm{THC}$ nachgewiesen wurde). Tabelle 14 zeigt die kumulativen Ausscheidungen von $\mathrm{COOH}-\mathrm{THC}$ in $\mu \mathrm{g} /$ Sammelintervall aller 43 Probanden während der fünf Urinsammelintervalle sowie für den gesamten Zeitraum von 24 Stunden.

Tab. 14 Ausscheidung von $\mathrm{COOH}-\mathrm{THC}$ aller Probanden in den Urin

\begin{tabular}{cccccc}
\hline Sammelintervall [h] & \multicolumn{5}{c}{ COOH-THC-Urinausscheidung [ug/Sammelintervall] } \\
\hline & Median & Minimum & 25. Perzentile & 75. Perzentile & Maximum \\
\cline { 2 - 6 } $0-3$ & 0.9 & 0.0 & 0.2 & 3.5 & 17.4 \\
$3-6$ & 12.2 & 0.3 & 5.2 & 23.4 & 100.0 \\
$6-9$ & 25.5 & 1.1 & 14.4 & 44.3 & 102.6 \\
$9-12$ & 16.1 & 0.0 & 10.0 & 26.6 & 60.7 \\
$12-24$ & 33.3 & 3.8 & 21.8 & 61.1 & 159.4 \\
$0-24$ & 109.4 & 6.1 & 70.5 & 158.3 & 410.8 \\
\hline
\end{tabular}


Über 24 Stunden lag die mediane Ausscheidung von COOH-THC aller Versuchsteilnehmer bei $109.4 \mu \mathrm{g}$ (Bereich 6.1 - 410.8 $\mu \mathrm{g}$ ). Damit wurden im Urin, in Form des carboxylierten Metaboliten, bezogen auf die Dosis von $15 \mathrm{mg}$ (entsprechend $15000 \mu \mathrm{g}$ ), nur 0.73\% (Bereich $0.04-2.74 \%$ ) ausgeschieden. Die Ausscheidungsraten aller Probanden in den fünf Sammelintervallen sind in Abbildung 20 illustriert.

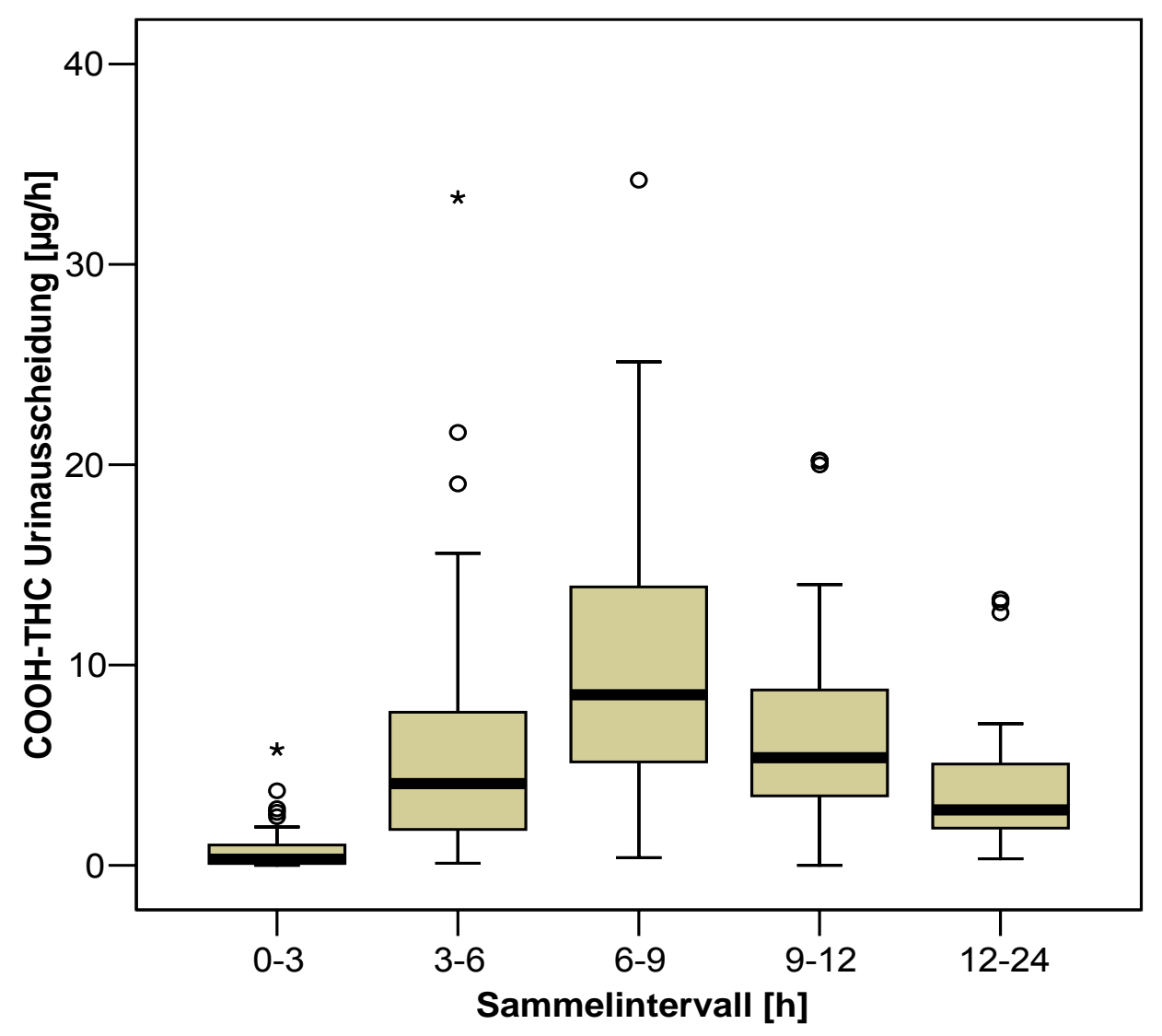

Abb. 20 Urinausscheidung von $\mathrm{COOH}-\mathrm{THC}$ in den fünf Sammelintervallen

Es wird deutlich, dass die Ausscheidung des $\mathrm{COOH}-\mathrm{THC}$ zunächst anstieg, ihr Maximum im Intervall von 6 - 9 Stunden erreichte und anschließend wieder abfiel. Die maximalen Urinkonzentrationen von $\mathrm{COOH}-\mathrm{THC}$ traten also 3 bis 6 Stunden verzögert zu den maximalen Plasmakonzentrationen von THC auf. Mögliche Zusammenhänge zwischen der quantitativen Ausscheidung von $\mathrm{COOH}-\mathrm{THC}$ in den Urin und den molekulargenetischen und demographischen Daten der Studienteilnehmer wurden untersucht. Diesbezüglich ergab sich keine Abhängigkeit der kumulativen Urinausscheidung vom Geschlecht, Alter, Körpergewicht und BMI. 


\subsubsection{Urinausscheidung von $\mathrm{THC}-\mathrm{COOH}$ in Abhängigkeit vom CYP2C9-Genpolymorphismus}

Zunächst wurde die Ausscheidung von $\mathrm{COOH}-\mathrm{THC}$ in den Sammelintervallen im Hinblick auf die Zahl an CYP2C9*2-Allelen analysiert. In allen Sammelintervallen schieden homozygote Träger des CYP2C9*2-Allels mehr $\mathrm{COOH}-\mathrm{THC}$ aus, als Personen, die keines dieser Allele trugen (Abbildung 21).

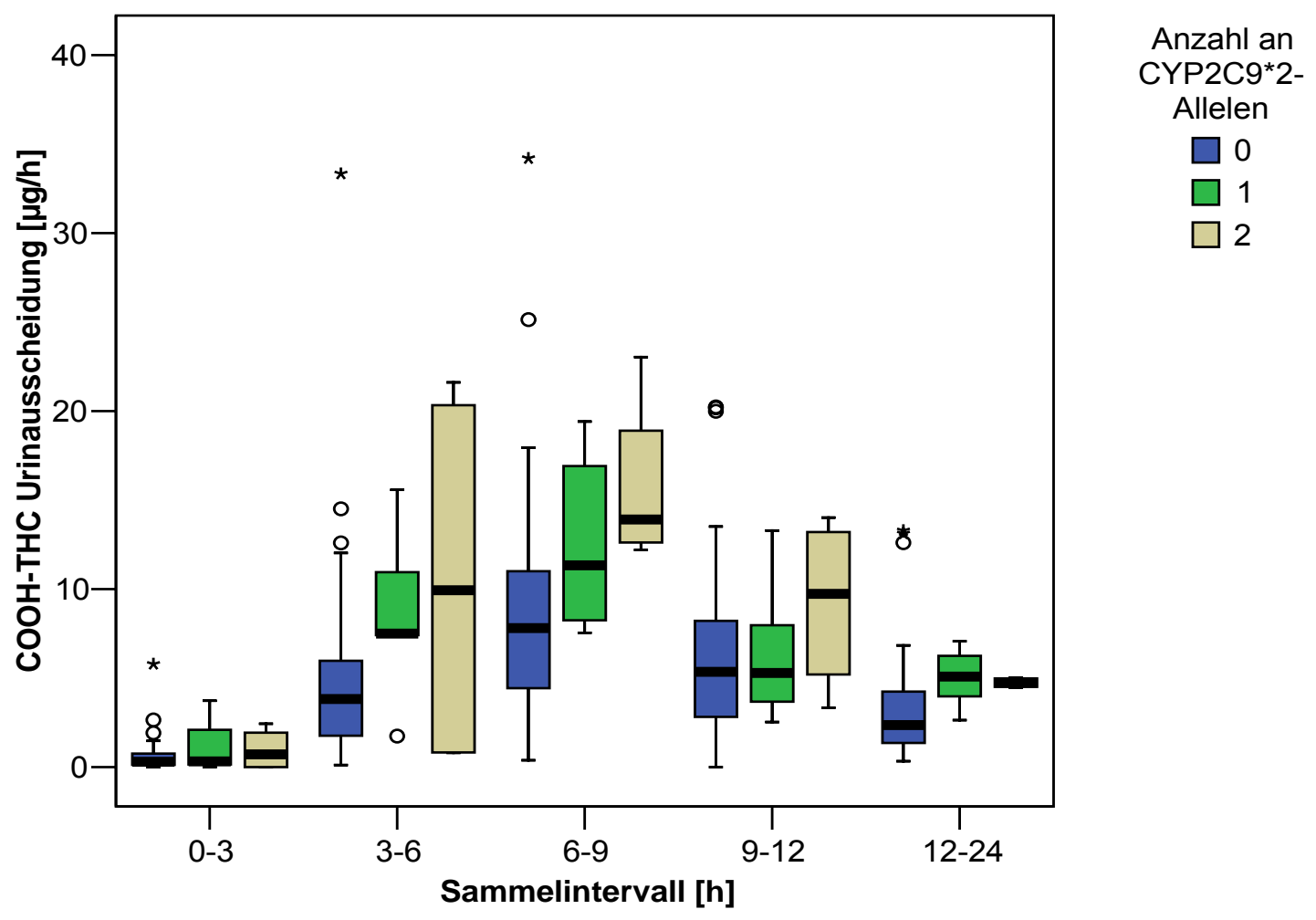

Abb. 21 Ausscheidung von $\mathrm{COOH}-\mathrm{THC}$ in den Urin in Abhängigkeit der Zahl an CYP2C9*2-Allelen

Mit Ausnahme des letzten Sammelintervalls wiesen diejenigen Probanden innerhalb des Intervalls die größte Ausscheidung von $\mathrm{COOH}-\mathrm{THC}$ auf, welche homozygote Träger des Allels CYP2C9*2 waren. Bei diesen Probanden war die mediane Urinausscheidung von $\mathrm{COOH}-\mathrm{THC}$ umso größer, je mehr CYP2C9*2-Allele sie trugen. Der festgestellte Trend verdeutlicht sich, wenn man die Ausscheidung kumulativ über 24 Stunden darstellt (Abbildung 22). 


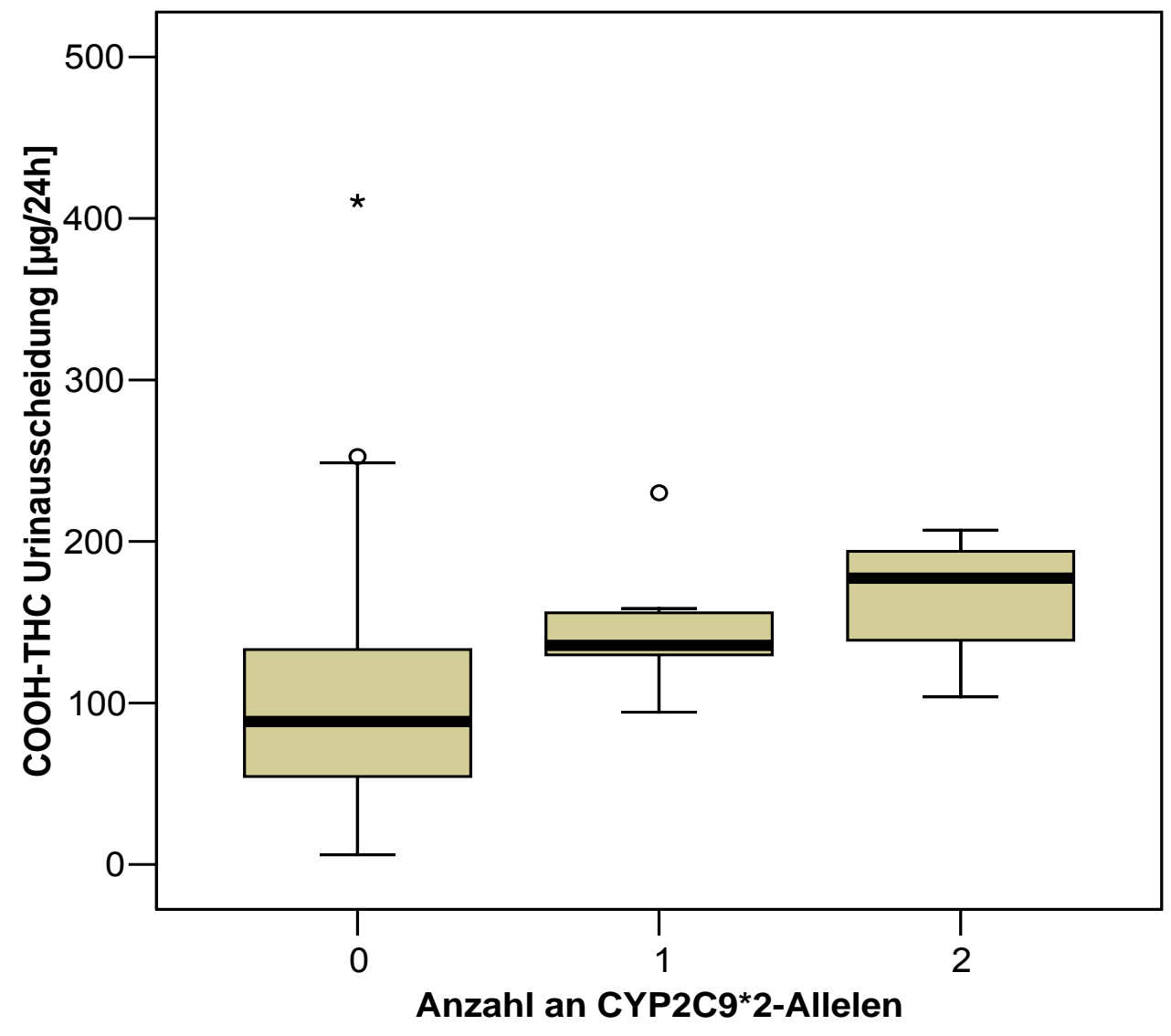

Abb. 22 Kumulative Urinausscheidung von $\mathrm{COOH}-\mathrm{THC}$ in Abhängigkeit der Zahl an CYP2C9*2-Allelen; 0,1 und 2 CYP2C9*2-Allele mit $n=32, n=7$ und $n=4$

Im Hinblick auf das CYP2C9*3-Allel ist, ähnlich zur vorherigen Betrachtung, in Abbildung 23 die Ausscheidung von $\mathrm{COOH}-\mathrm{THC}$ nach der Zahl an CYP2C9*3Allelen unterteilt. Auch hier wurden die verschiedenen Urinsammelintervalle berücksichtigt. 


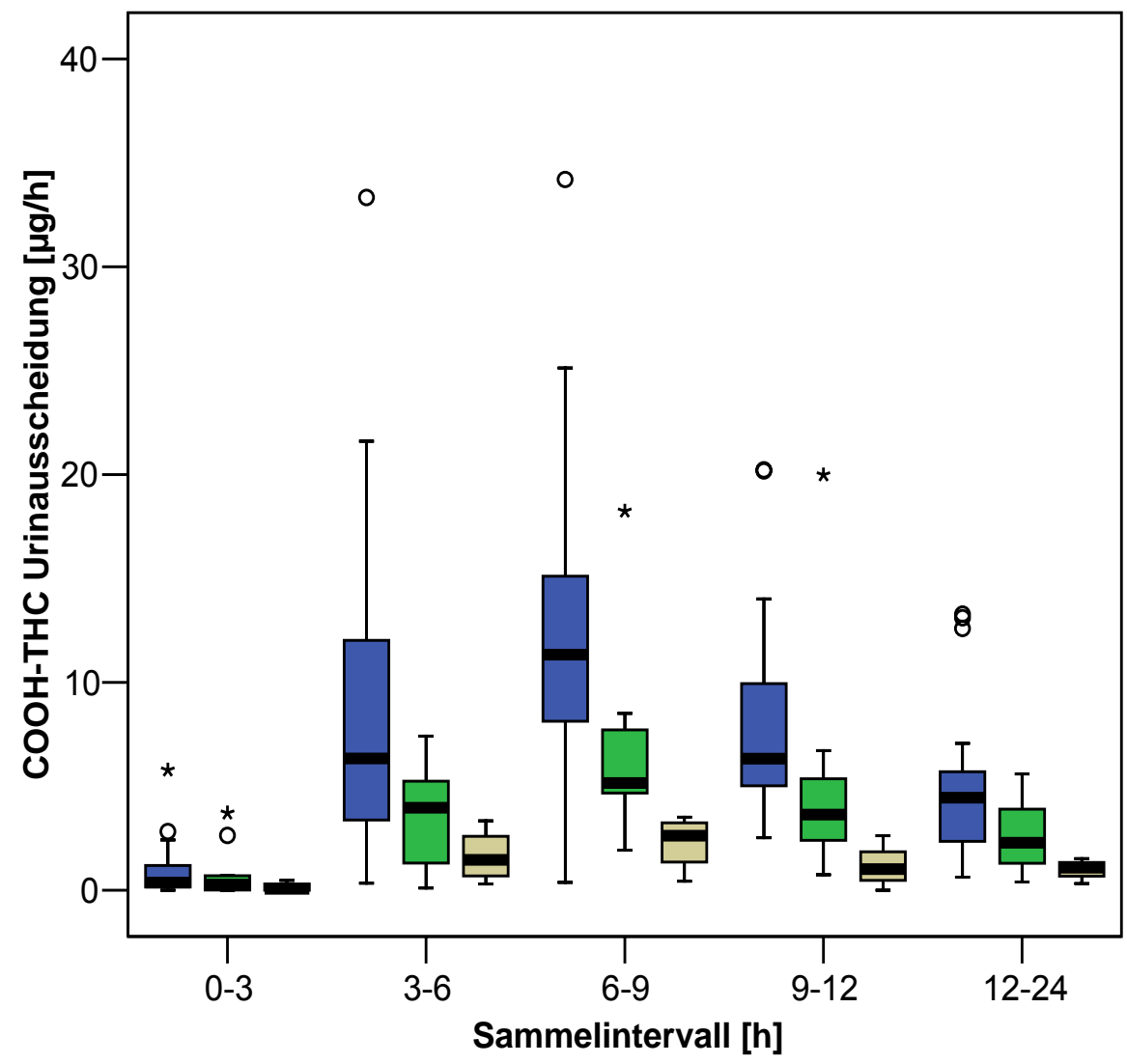

Anzahl an CYP2C ${ }^{*} 3-$ Allelen

$\square 0$

$\square 1$

$\square 2$

Abb. 23 Ausscheidung von $\mathrm{COOH}-\mathrm{THC}$ in den Urin in Abhängigkeit der Zahl an CYP2C9*3-Allelen

Es ist zu erkennen, dass sich in Abhängigkeit des Allels CYP2C9*3 Unterschiede in der Urinausscheidung von $\mathrm{COOH}-\mathrm{THC}$ ergaben. Träger des Genotyps CYP2C9*3/*3 schieden in allen Sammelintervallen am wenigsten $\mathrm{COOH}-\mathrm{THC}$ aus. Innerhalb jedes einzelnen Intervalls nahm die mediane Ausscheidungsrate pro Stunde ab, je mehr CYP2C9*3-Allele die Probanden trugen. Dies wird auch im Hinblick auf die Menge an $\mathrm{COOH}-\mathrm{THC}$, die über 24 Stunden eliminiert wurde, deutlich. Die dazugehörigen Ergebnisse sind in Abbildung 24 illustriert. 


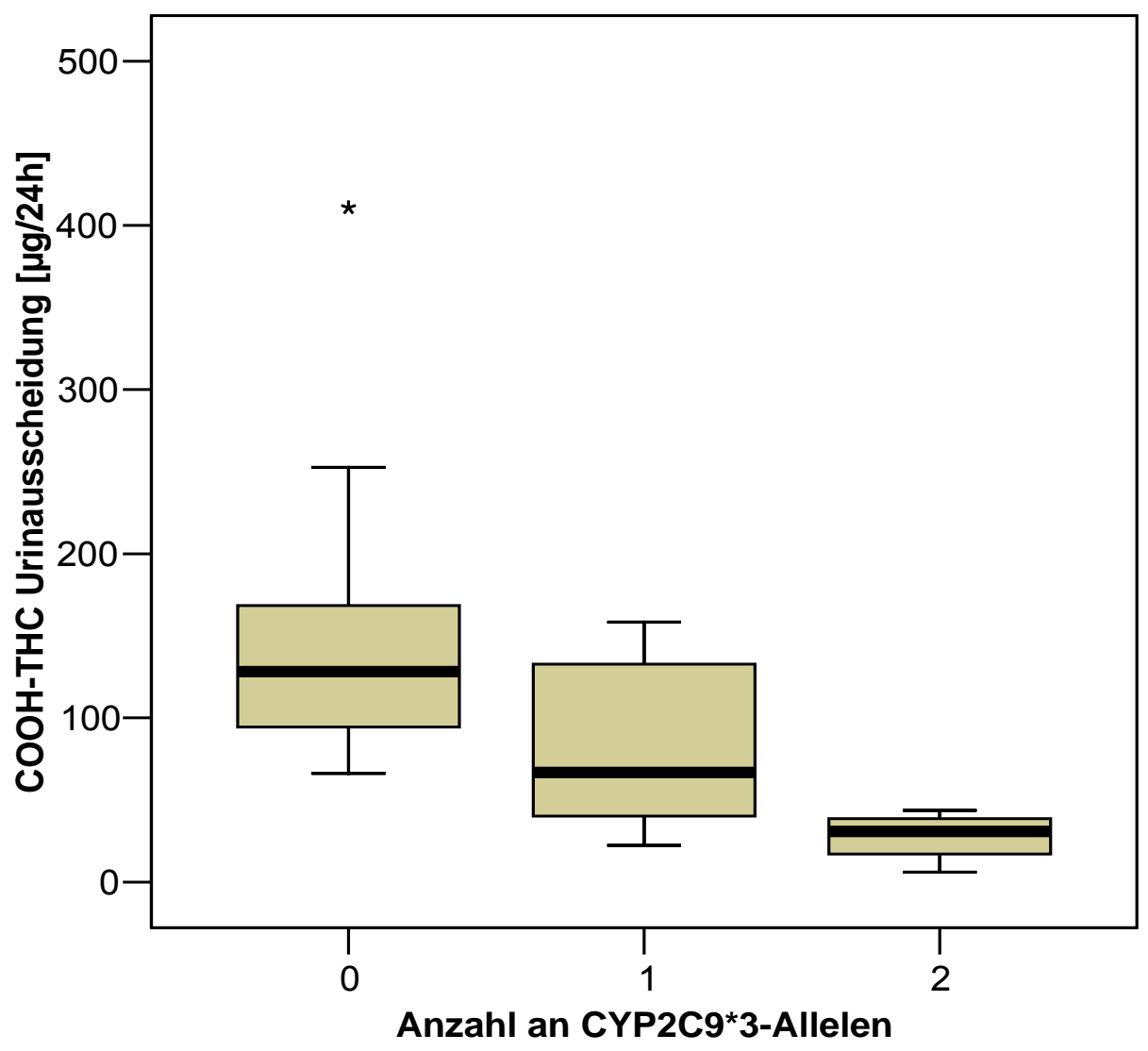

Abb. 24 Kumulative Urinausscheidung von $\mathrm{COOH}-\mathrm{THC}$ in Abhängigkeit der Zahl an CYP2C9*3-Allelen; 0, 1 und 2 CYP2C9*3-Allele mit $n=29, n=10$ und $n=4$

Die zuvor graphisch illustrierten Ergebnisse sind in Tabelle 15 als Zahlenwerte zusammenfassend und nach den Allelen CYP2C9*2 und -*3 differenziert dargestellt.

Tab. 15 Urinausscheidung von $\mathrm{COOH}-\mathrm{THC}$ über 24h in Abhängigkeit der Zahl an CYP2C9*2- und -*3-Allelen

\begin{tabular}{|c|c|c|c|c|c|}
\hline \multirow{2}{*}{$\begin{array}{l}\text { Zahl der } \\
\text { CYP2C9*2- } \\
\text { Allele }\end{array}$} & \multicolumn{2}{|c|}{$\begin{array}{l}\text { COOH-THC } \\
\text { Urinausscheidung }[\mu \mathrm{g} / 24 \mathrm{~h}]\end{array}$} & \multirow{2}{*}{$\begin{array}{l}\text { Zahl der } \\
\text { CYP2C9*3- } \\
\text { Allele }\end{array}$} & \multicolumn{2}{|c|}{$\begin{array}{l}\text { COOH-THC } \\
\text { Urinausscheidung [ } \mu \mathrm{g} / 24 \mathrm{~h}]\end{array}$} \\
\hline & Median & Q1 - Q3 & & Median & Q1 - Q3 \\
\hline 0 & 88.6 & $50.3-133.3$ & 0 & 128.2 & $93.8-171.2$ \\
\hline 1 & 135.7 & $124.9-158.3$ & 1 & 66.8 & $39.8-132.9$ \\
\hline 2 & 177.3 & $121.4-200.5$ & 2 & 30.9 & $11.6-41.2$ \\
\hline
\end{tabular}

Q1 entspricht dem 0.25-Quantil; Q2 entspricht dem 0.75-Quantil

Wie bereits erwähnt, stiegen die Mediane der Urinausscheidung von $\mathrm{COOH}-\mathrm{THC}$ über 24 Stunden mit der Zahl an CYP2C9*2-Allelen an. Die Mediane betrugen 88.6, 135.7 und $177.3 \mu \mathrm{g} / 24 \mathrm{~h}$ für 0,1 und 2 CYP2C9*2-Allele. Dieser Unterschied war 
statistisch signifikant ( $p=0.004$, Jonckheere-Terpstra-Trend-Test). Im Trend genau umgekehrt war die Eliminierung von $\mathrm{COOH}-\mathrm{THC}$ umso geringer, je mehr CYP2C9*3-Allele die Versuchsteilnehmer trugen. Die Mediane lagen für Träger von 0,1 und 2 CYP2C9*3-Allelen bei 128.2, 66.8 und $30.9 \mu \mathrm{g} / 24 \mathrm{~h}$. Auch dieser Unterschied war statistisch signifikant $(p<0.001)$. Ergänzend sind in Tabelle 16 die ausgeschiedenen Mengen differenziert nach den tatsächlichen Allelkombinationen dargestellt.

Tab. 16 Ausscheidung von $\mathrm{COOH}-\mathrm{THC}$ in 24 Stunden, nach CYP2C9-Genotypen

\begin{tabular}{ccc}
\hline CYP2C9-Genotyp & \multicolumn{2}{c}{ Kumulative Ausscheidung von THC-COOH in $\boldsymbol{\mu g} / \mathbf{2 4 h}$} \\
\cline { 2 - 3 }${ }^{*} 1 /{ }^{*}$ & Median & Q1 - Q3 \\
${ }^{*} /{ }^{*} 2$ & 118.3 & $87.7-167.6$ \\
${ }^{*} /{ }^{*} 2$ & 135.2 & $117.3-172.6$ \\
${ }^{*} 1 /{ }^{*} 3$ & 177.3 & $121.4-200.5$ \\
${ }^{*} /{ }^{*} 3$ & 63.1 & $39.3-110.6$ \\
${ }^{*} /{ }_{3}$ & 158.3 & \\
\hline
\end{tabular}

Q1 entspricht dem 0.25-Quantil; Q2 entspricht dem 0.75-Quantil 


\subsubsection{Urinausscheidung von $\mathrm{THC}-\mathrm{COOH}$ in Abhängigkeit vom CYP3A5-Genpolymorphismus}

Bei der folgenden Betrachtung des CYP3A5-Genpolymorphismus wurde, wie bereits hinsichtlich des CYP2C9-Genotyps, ausschließlich die Subgruppe der 19 Probanden, die den $C Y P 2 C 9{ }^{*} /{ }^{*} 1$-Genotyp trugen, untersucht. Die kumulative Ausscheidung von THC-COOH ergab bei den 13 CYP3A5*3/*3-Trägern (keine CYP3A5-Aktivität) im Median höhere Werte als bei den 6 Trägern der enzymatisch aktiven Variante CYP3A5*1/*3 (Abbildung 25)

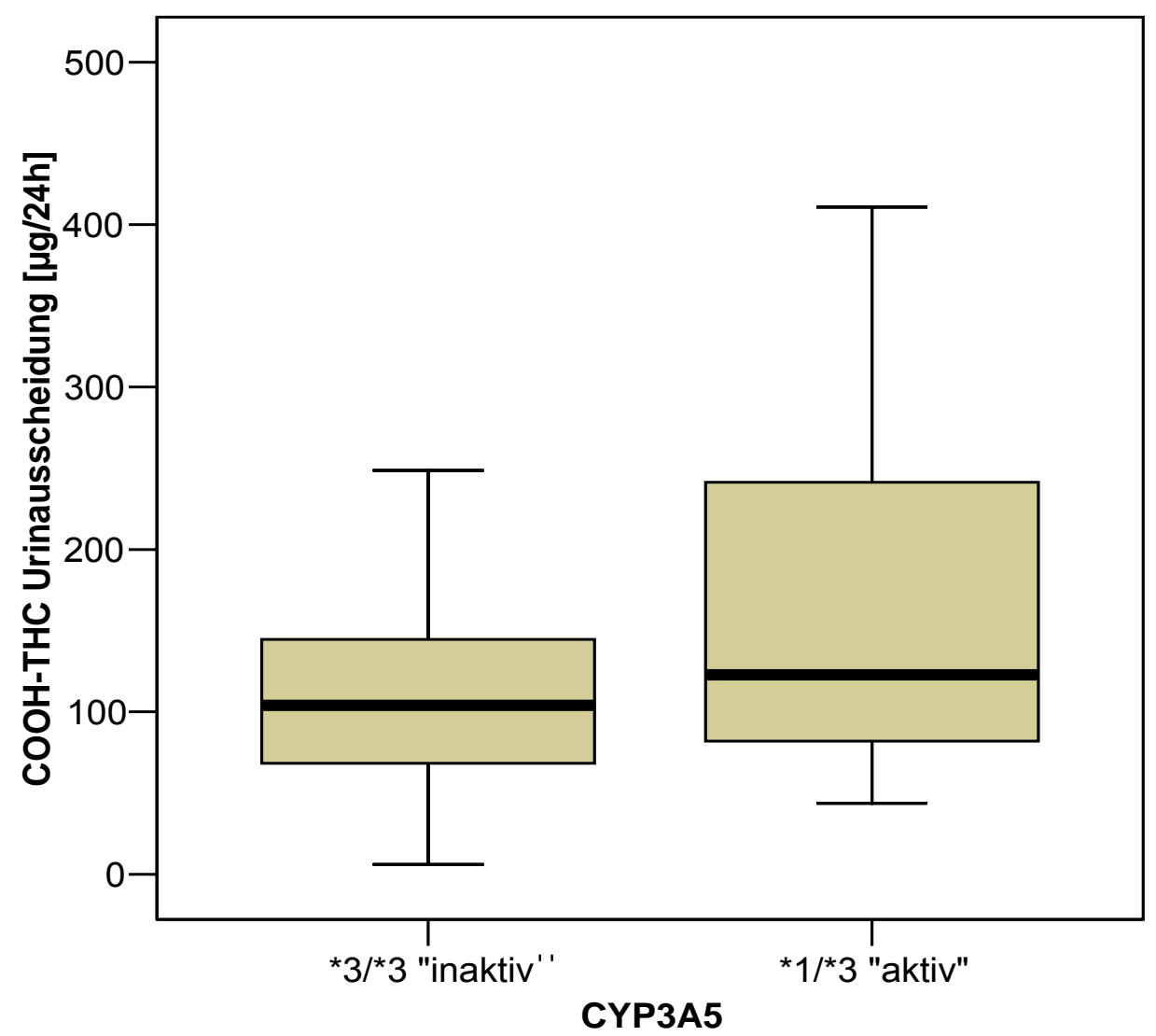

Abb. 25 Kumulative Ausscheidung von $\mathrm{COOH}-\mathrm{THC}$ in den Urin in Abhängigkeit vom CYP3A5-Genpolymorphismus; CYP2C9*3/*3n $=13$, CYP2C9*1/*3 $n=6$

Die Mediane (Interquartilenbereich) lagen für die Träger des inaktiven Genotyps CYP3A5*3/*3 bei $104.0 \mu \mathrm{g} / 24 \mathrm{~h}(66.2$ - 153.4) und für diejenigen des aktiven Genotyps CYP3A5*1/*3 bei $122.8 \mu \mathrm{g} / 24 \mathrm{~h}$ (76.3 - 247.0). Dieser Unterschied war jedoch statistisch nicht signifikant (Mann-Whitney-U-Test). 


\subsubsection{Ausscheidung von THC-COOH in Abhängigkeit vom OATP1B1- Genpolymorphismus}

Die OATP1B1-Transportproteine (organic anion transporter peptide) transportieren starke Säuren. Da möglicherweise auch das $\mathrm{COOH}-\mathrm{THC}$ zu den Substraten dieses Transporters zählt, wurden die Genpolymorphismen des OATP1B1 explorativ im Hinblick auf die 24h-Urinausscheidung von $\mathrm{COOH}-\mathrm{THC}$ analysiert. Die kumulativen Urinausscheidungen standen in keinem Zusammenhang mit den beiden analysierten Genvarianten $130_{\text {Asparagin }}$ und $174_{\text {Alanin }}$ (Abbildungen 26 und 27).

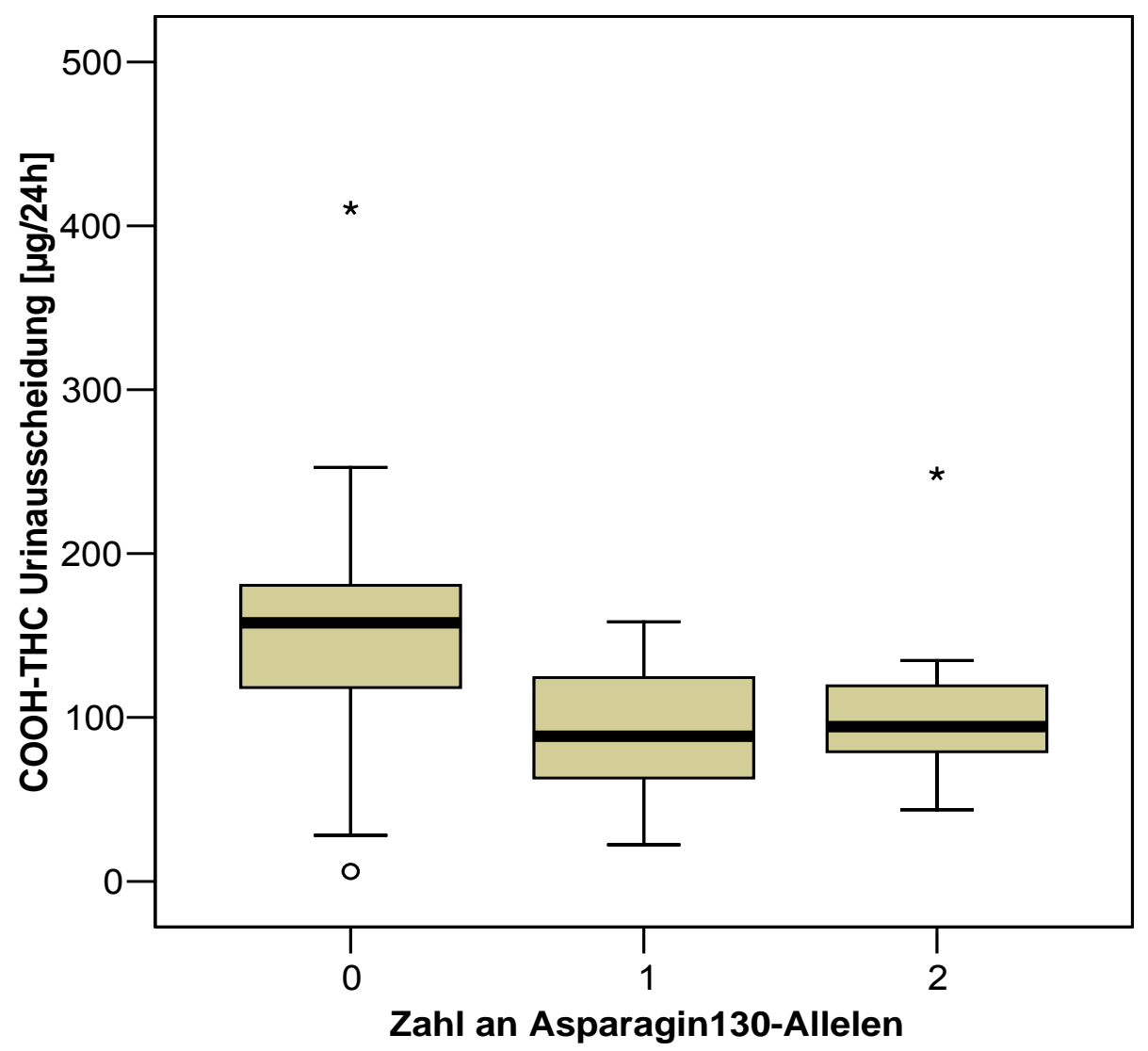

Abb. 26 Kumulative Urinausscheidung von $\mathrm{COOH}-\mathrm{THC}$ in Abhängigkeit der Zahl an $130_{\text {Asparagin }}$-Allelen 


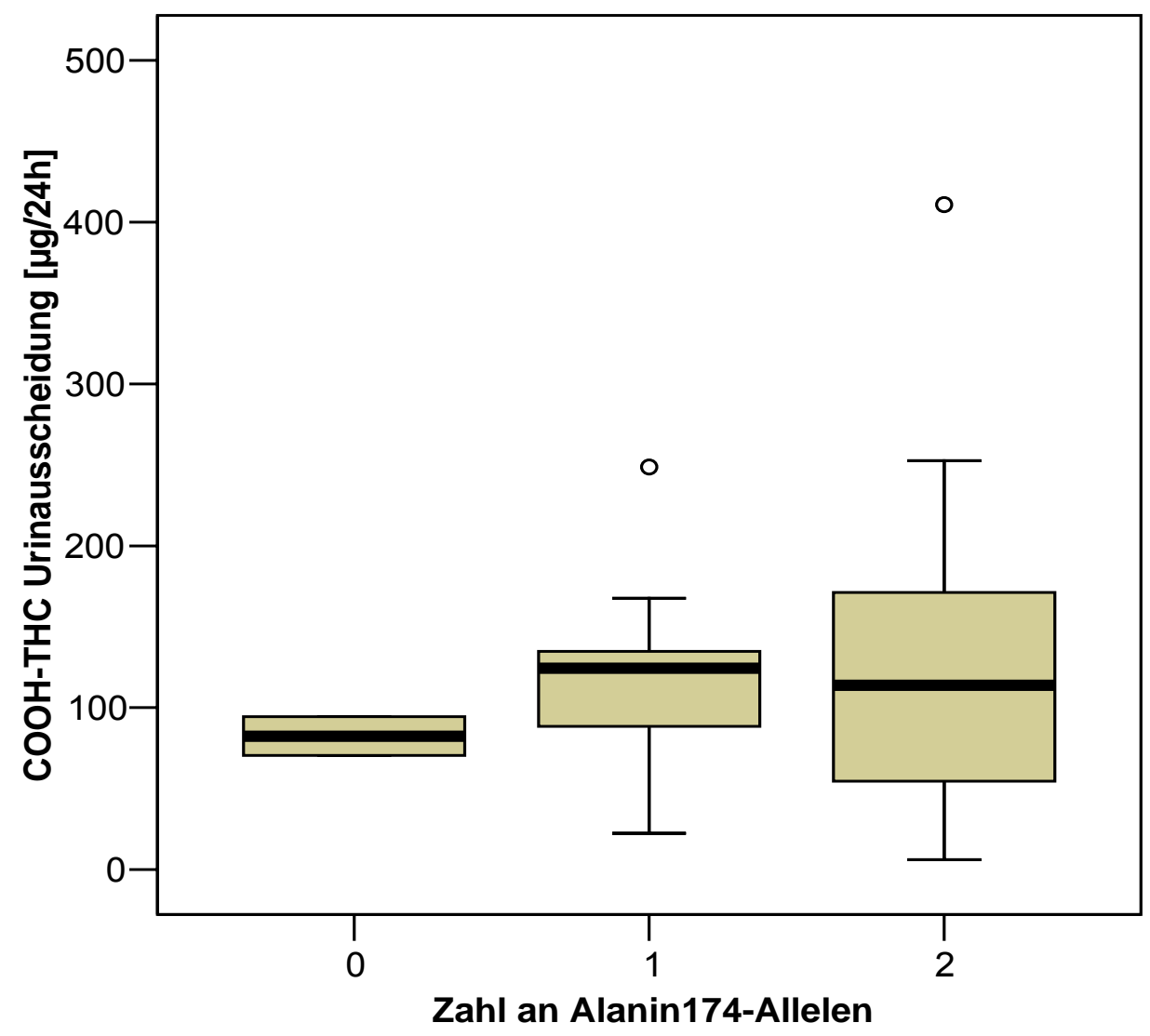

Abb. 27 Kumulative Urinausscheidung von $\mathrm{COOH}-\mathrm{THC}$ in Abhängigkeit der Zahl an $174_{\text {Alanin }}$-Allelen des Gens OATP1B1

\subsection{Nebenwirkungen von Dronabinol}

Für Dronabinol sind eine Reihe von Nebenwirkungen bekannt. In unserer Studie wurden neuropsychiatrische und vegetative Nebenwirkungen durch die Fragebögen 1 und 2 erfasst (Kapitel 3.8.1). Integriert in Fragebogen 2 wurde der Müdigkeitsgrad der Studienteilnehmer mittels der Stanford-Sedierungs-Skala dokumentiert (Kapitel 3.8.2). Der Einfluss von Dronabinol auf die Kreislaufparameter Blutdruck und Herzfrequenz wurde anhand regelmäßiger Messungen erfasst (Kapitel 3.8.3).

\subsubsection{Nebenwirkungen unter THC (Fragebögen 1 und 2)}

Zur Bearbeitung der Fragebögen 1 und 2 (Fragebögen im Anhang) hatten die Probanden zu jeder Fragestellung auf einer Ratingskala jeweils eine Zahl von 0 (nein/ trifft gar nicht zu) bis 5 (trifft sehr stark zu) anzukreuzen. Auch Freitextangaben waren gestattet. 
Die bekannten Akutnebenwirkungen wie Mundtrockenheit und konjunktivale Injektion kamen auch im Rahmen unserer Untersuchung vor (bei 41.9\% bzw. 30.2\% der Probanden). Weiterhin traten gelegentlich trockene, gereizte Augen (41.9\%), kalte Hände und Füße (72.1\%), Schwindel (44.2\%), Kopfschmerzen (44.2\%) und Übelkeit (25.6\%) auf. Bei den psychischen Nebenwirkungen ließen sich das Erleben eines Hochgefühls („High“) oder eine veränderte Zeitwahrnehmung beobachten (46.5\% bzw. 48.8\%). Die Mehrzahl der Probanden berichtete unter dem Einfluss der Studienmedikation zeitweise über Konzentrationsschwierigkeiten und ein allgemeines Müdigkeitsgefühl (81.4\% bzw. $86.0 \%)$. In der Freitextangabe beschrieben zwei Probanden ein „Druckgefühl im Kopf“ sowie fünf der Probanden eine "dumpfe, gedämpfte und verlangsamte Wahrnehmung“ ihrer Umgebung. Drei der Studienteilnehmer empfanden eine gewisse "Sensibilisierung auf Umweltreize" und eine „vermehrte Geräuschwahrnehmung". "Gedankensprünge" traten bei zwei Probanden auf. Ein Proband stellte ein passageres Gefühl des „Kontrollverlusts“ fest.

\subsubsection{Sedierungs-Score}

Als eine häufige akute Nebenwirkung von Dronabinol wird die Sedierung genannt. Mit Hilfe der siebenstufigen Stanford Sleepiness Scale (Stanford-Sedierungs-Skala) sollte, zusätzlich zur Erfassung der Müdigkeit über den POMS-Test (Kapitel 3.9.5), der Schläfrigkeitsgrad der Probanden abgeschätzt werden. Dazu wurde die Schläfrigkeit der Probanden am Verumtag zehnmal und an den drei weiteren Studientagen dreimal bewertet. Die Beurteilungsskala reichte dabei von "1" (sich sehr aktiv, vital, aufmerksam beziehungsweise sehr wach fühlen) bis "7“ (nicht länger gegen den Schlaf ankämpfend baldiges Einsetzen des Schlafes, beziehungsweise traumartige Gedanken; Kopie der Skala im Anhang). Die Einordnung der Probanden in diese Skala erfolgte stets sowohl durch den Versuchsteilnehmer selbst, als auch durch den Untersucher.

In Abbildung 28 sind die Einzelwerte aller Probanden sowie je eine Kurve der Mittelwerte des Schläfrigkeitsniveaus in Abhängigkeit der Anzahl der CYP2C9*3-Allele gezeigt. Es handelte sich hier um die Selbsteinschätzung der Studienteilnehmer. Abbildung 29 illustriert auf die gleiche Weise den Grad der Schläfrigkeit. Es handelt sich an dieser Stelle jedoch um die Einschätzung des 
Untersuchers. Letzteres entspricht der von den Autoren vorgesehenen Verwendung der Stanford Sleepiness Scale als Fremdbeobachtungsinstrument.

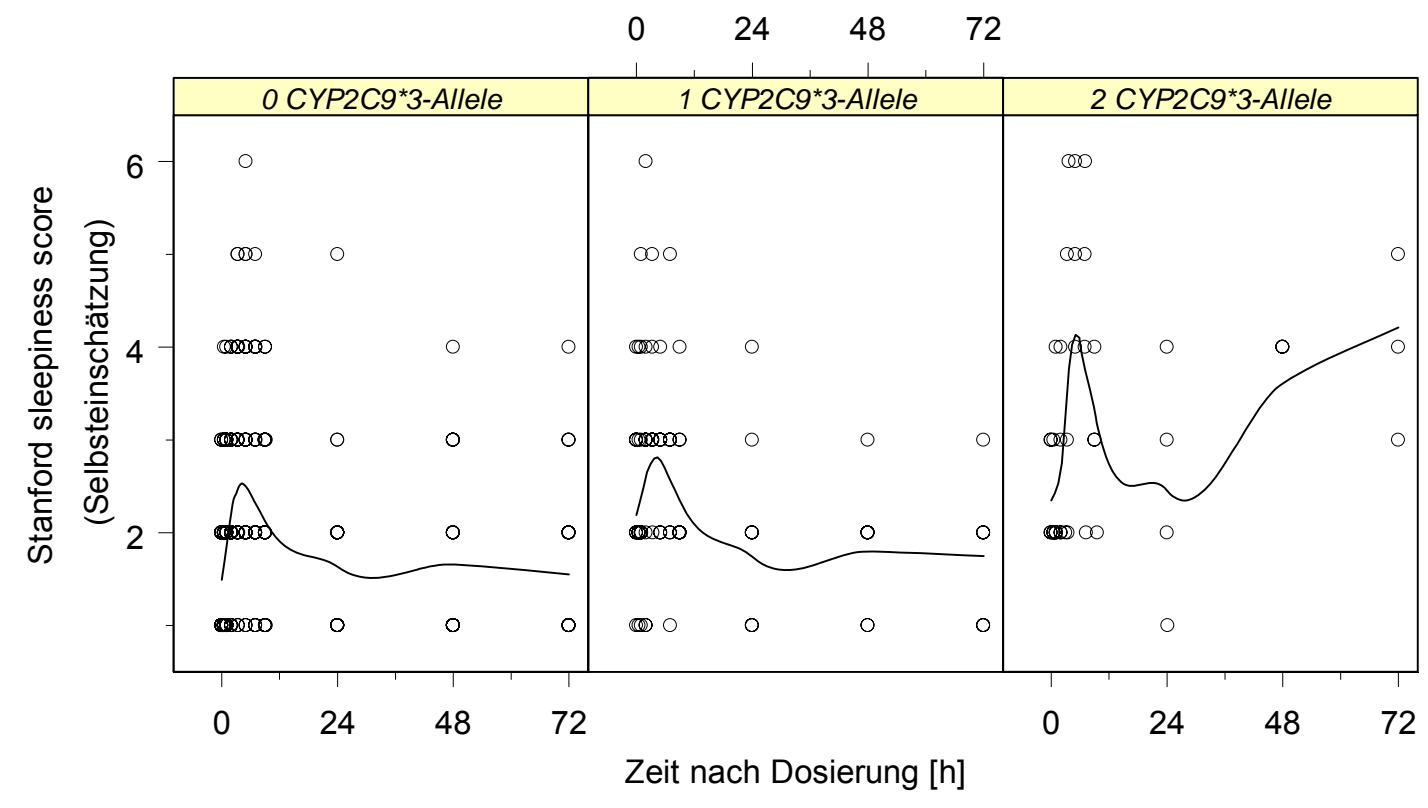

Abb. 28 Stanford-Sedierungs-Skala in Abhängigkeit der Zahl an CYP2C9*3-Allelen

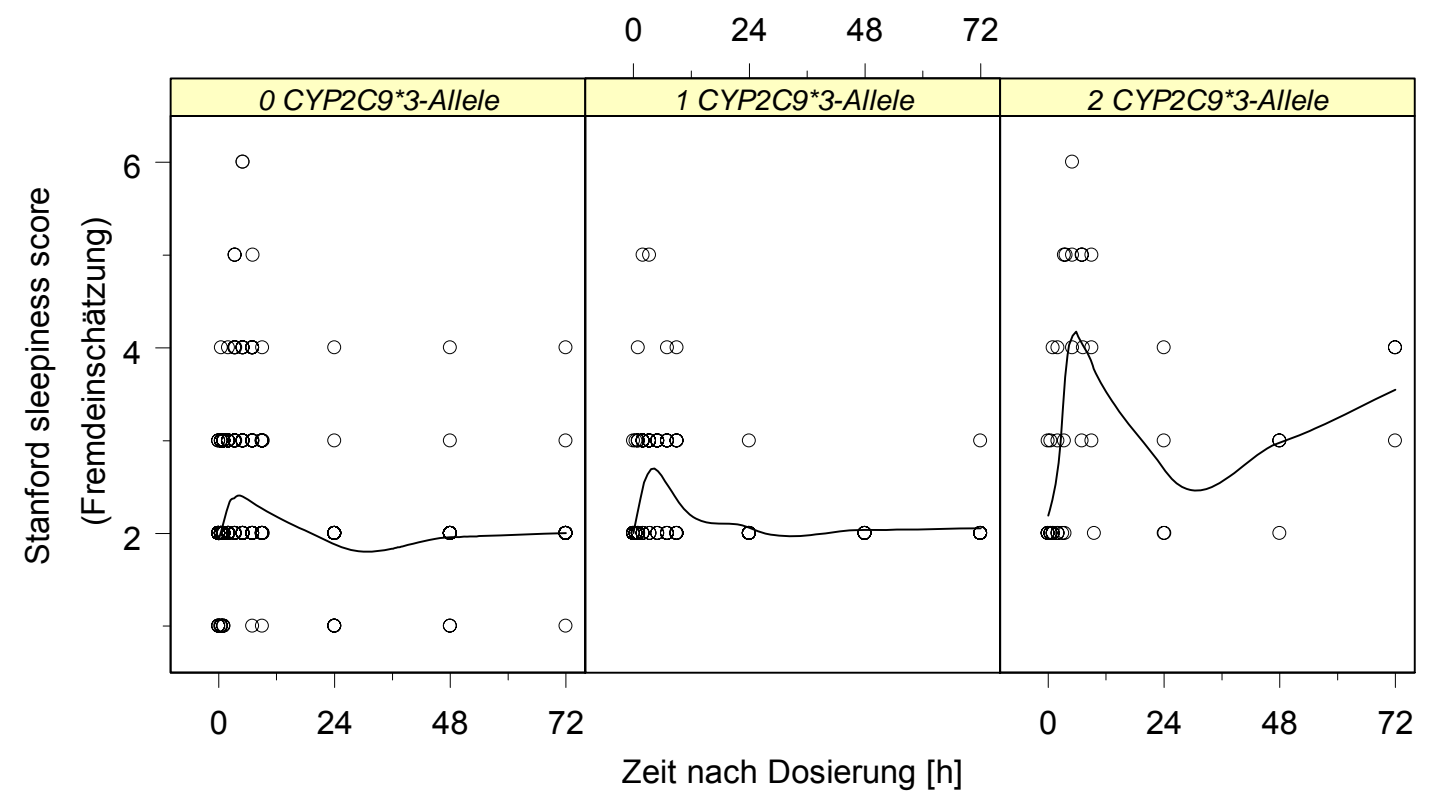

Abb. 29 Stanford-Sedierungs-Skala in Abhängigkeit der Zahl an CYP2C9*3-Allelen 
Die Ergebnisse der Selbsteinschätzung deckten sich weitgehend mit denen der Fremdeinschätzung. Es kam medikamentenbedingt zu einem Anstieg der Werte auf der Stanford-Sedierungs-Skala, wobei entsprechend dem Studiendesign, welches bei der Konzentration auf die pharmakokinetischen Analysen keine Placebokontrolle vorgesehen hat, Placeboeffekte oder unspezifische Ermüdungseffekte nicht mit endgültiger Sicherheit ausgeschlossen werden können. Tendenziell wurde deutlich, dass die Probanden zu höheren Werten neigten, je mehr CYP2C9*3-Allele sie trugen. Sehr deutliche Unterschiede zeigte diesbezüglich der Vergleich der Probandenkohorte mit zwei CYP2C9*3-Allelen gegenüber denen mit null bzw. einem -*3-Allel. Der Zeitraum der verstärkten Müdigkeit stimmte in etwa mit dem der erhöhten Blutplasmaspiegel der psychoaktiven Substanzen THC und OH-THC überein.

\subsubsection{Vegetative Nebenwirkungen anhand von Blutdruck und Herzfrequenz}

Bei dem Konsum von Cannabisprodukten, also auch unter der Therapie mit Dronabinol, kann es zu Schwankungen des Blutdrucks und der Herzfrequenz kommen. Diese sollten in unserer Studie insbesondere durch regelmäßige Messungen des Blutdrucks und der Herzfrequenz erfasst werden.

Unter dem Einfluss der Studienmedikation ließ sich ein Anstieg der Herzfrequenz beobachten (Abbildung 30). Spätestens 24 Stunden nach Medikamentengabe hatten sich die Werte wieder normalisiert. Ein Genotyp-abhängiger Effekt ist nicht zu erkennen. Der nach unten schwankende Verlauf der CYP2C ${ }^{*} 3 /{ }^{*} 3-$ Träger wird bei der geringen Fallzahl $(n=4)$ als Zufallsbefund interpretiert. 


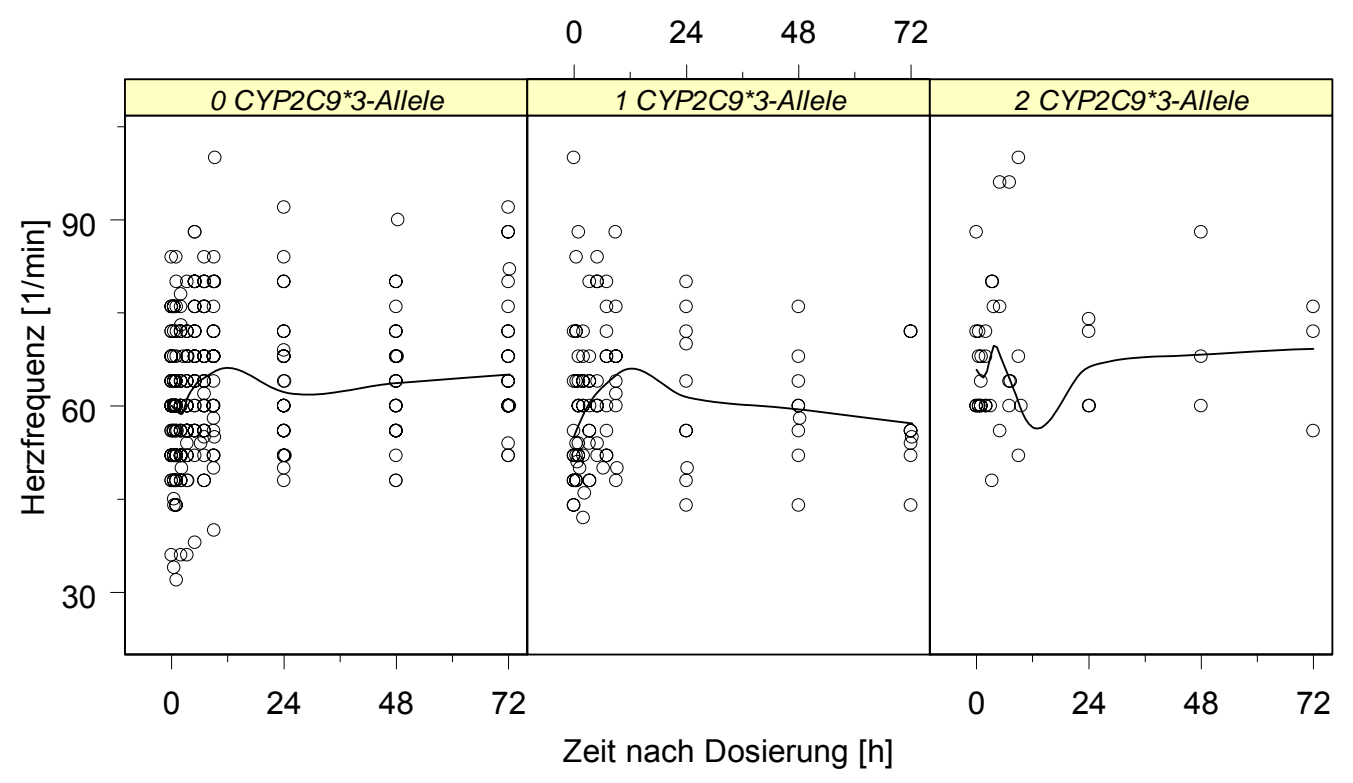

Abb. 30 Herzfrequenzen aller Probanden in Abhängigkeit der Zahl an CYP2C9*3-Allelen

Bezüglich des Blutdrucks zeigten sich die Ergebnisse uneinheitlich. Die systolischen Blutdrücke fielen bei Probanden mit 0 und 2 CYP2C9*3-Allelen unter dem Einfluss von Dronabinol im Mittel leicht ab und kehrten innerhalb von 24 Stunden nach Medikamentengabe wieder auf ihre Ausgangswerte zurück. Bei Studienteilnehmern mit nur einem CYP2C9*3-Allel blieben die systolischen Blutdruckwerte während der gesamten Studiendauer unbeeinflusst (Abbildung 31).

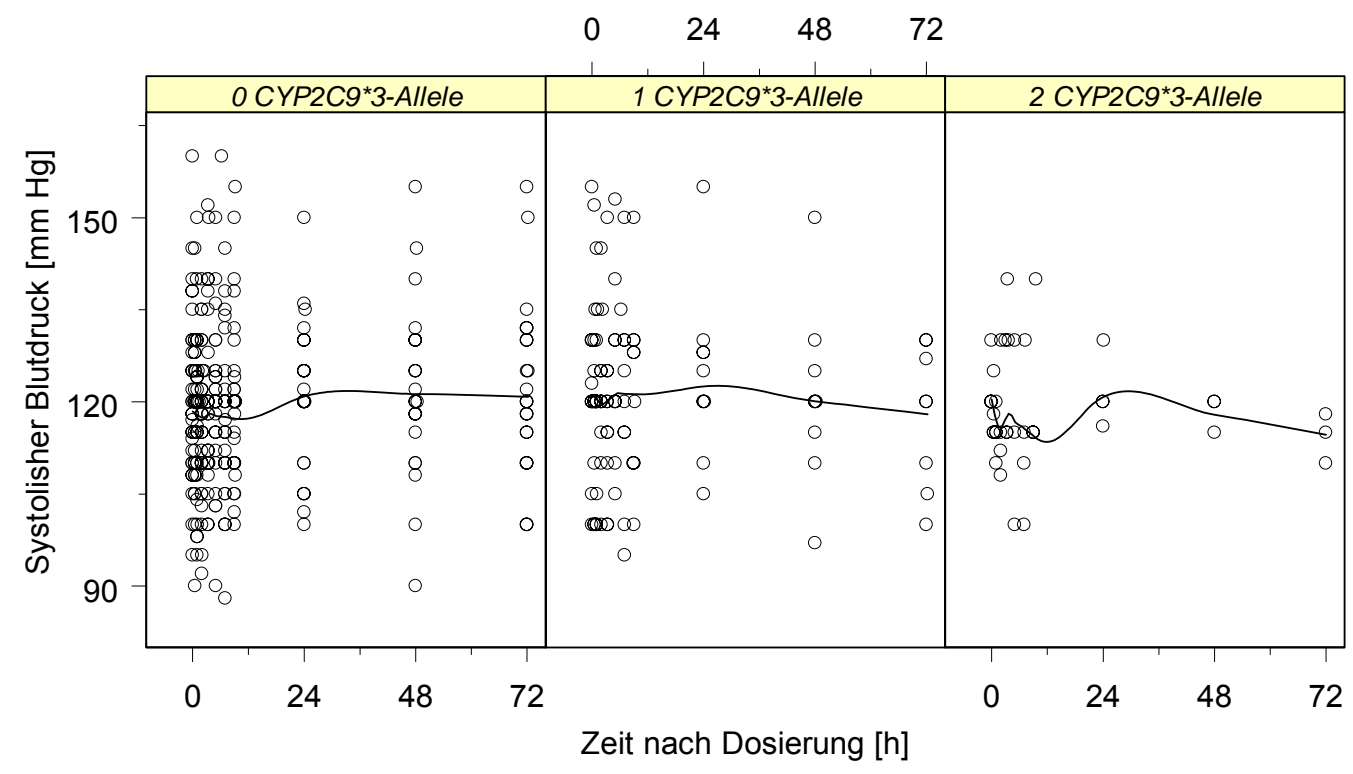

Abb. 31 Systolische Blutdrücke aller Probanden in Abhängigkeit der Zahl an CYP2C9*3-Allelen 
Betrachtet man die Werte für den diastolischen Blutdruck, zeigte sich innerhalb der ersten 24 Stunden nach Medikamentengabe bei Trägern von 0, 1 und 2 CYP2C9*3-Allelen ein leichtes Absinken. Am stärksten war dies bei jenen Probanden ausgeprägt, die nur 1 CYP2C9*3-Allel trugen (Abbildung 32). In der Gruppe der Träger von 2 CYP2C9*3-Allelen war, sowohl hinsichtlich des systolischen, als auch des diastolischen Blutdrucks nach einem initialen Absinken, zunächst wieder ein Anstieg, gefolgt von einem erneuten Abfall zu beobachten, was am ehesten als Zufallsbefund eingeordnet werden muss.

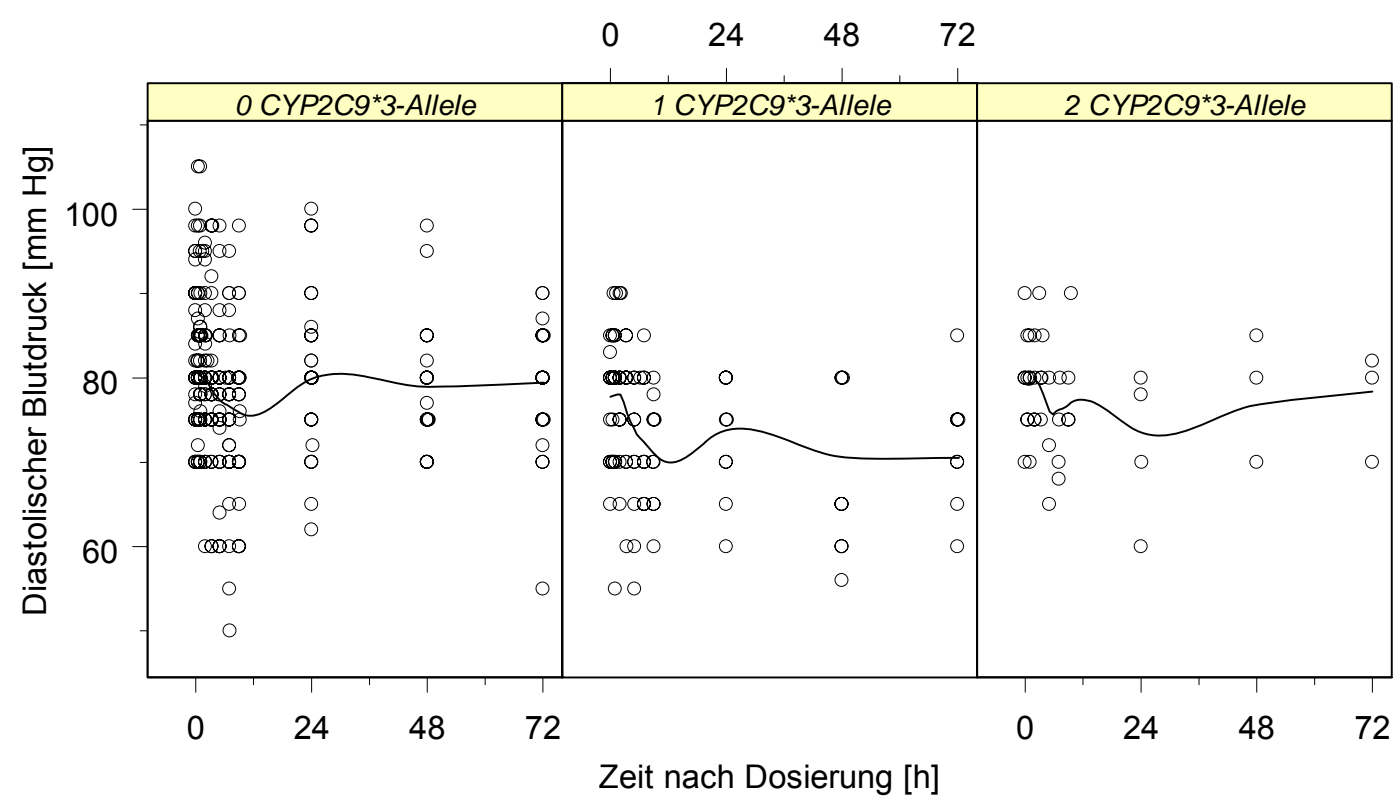

Abb. 32 Diastolische Blutdrücke aller Probanden in Abhängigkeit der Zahl an CYP2C9*3-Allelen

\subsection{Pharmakodynamik in Bezug zur Pharmakokinetik und -genetik}

Die Wirkungen von Dronabinol wurden anhand psychometrischer Messungen erfasst. Es sollte untersucht werden, ob es durch die Studienmedikation von $15 \mathrm{mg}$ Dronabinol zu Veränderungen in der Kognition und dem Befinden der Probanden kommt. Insbesondere sollten mögliche Zusammenhänge zwischen der Pharmakogenetik (CYP2C9-, CYP3A5- und OATP1B1-Polymorphismen) und der Pharmakodynamik, also den Wirkungen von Dronabinol, analysiert werden. Es wurden fünf wesentliche Bereiche untersucht, auf die THC nach der Literatur einen Einfluss haben kann: Die Reaktionsgeschwindigkeit (Reaktionstest), die Wahrnehmungsgeschwindigkeit (Flimmer- bzw. Verschmelzungsfrequenz), die Zeit- 
und Bewegungswahrnehmung (Zeit- und Bewegungsantizipation), die Gedächtnisleistung (fortlaufende visuelle Wiedererkennung) und die Stimmung (POMS).

\subsubsection{Reaktionstest (RT)}

Zur Erfassung der Reaktionszeit auf optische und akustische Reize wurde ein Reaktionstest eingesetzt. Den Probanden wurden abwechselnd Licht- und Tonsignale sowie Kombinationen davon vorgegeben. Die Studienteilnehmer mussten bei bestimmten kritischen Reizkombinationen mit einem Tastendruck reagieren. Abbildung 33 zeigt die mittleren Reaktionszeiten aller Probanden. Diese war definiert als Zeit zwischen dem Beginn des geforderten Reizes und dem Verlassen der Ruhetaste.

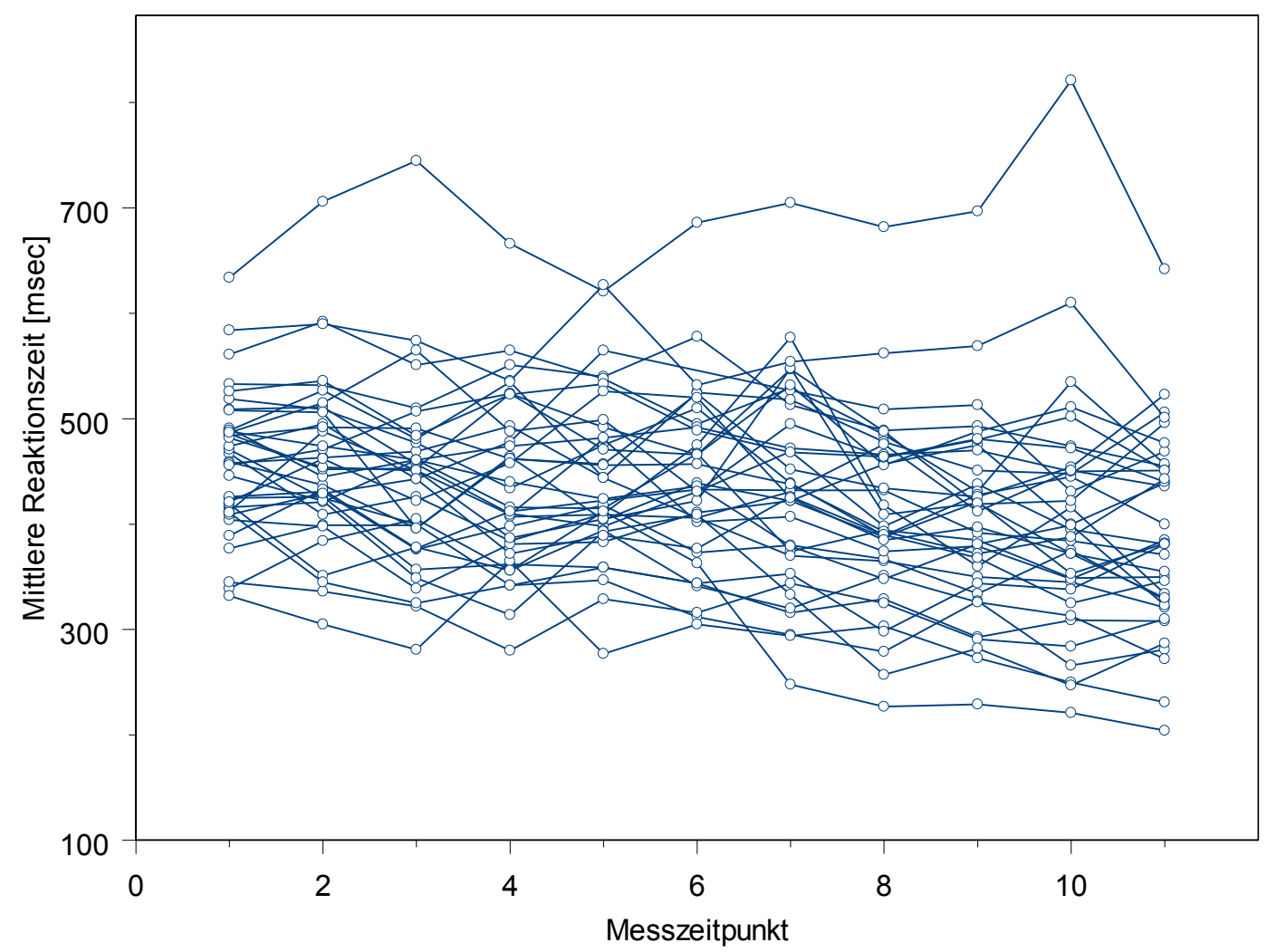

Abb. 33 Mittlere Reaktionszeiten aller Probanden; SD, Studientag; TP, Zeitpunkt; Messzeitpunkt $1=$ Baseline TP2, Messzeitpunkt 2 = Baseline TP4, Messzeitpunkt $3=$ Baseline TP8, Messzeitpunkt $4=$ Baseline TP11, Messzeitpunkt $5=$ SD1 TP2, Messzeitpunkt $6=$ SD1 TP4, Messzeitpunkt $7=$ SD1 TP8, Messzeitpunkt $8=$ SD1 TP11, Messzeitpunkt $9=$ SD2, Messzeitpunkt $10=$ SD3, Messzeitpunkt 11 = SD4; die Medikamentengabe erfolgte 60 min vor Messzeitpunkt 5. 
Es zeigte sich eine deutliche Streuung der Messwerte. Anhand der Kurve der Mittelwerte ließ sich keine Veränderung der Reaktionszeit nach erfolgter Medikamentengabe erkennen. Im Gegensatz zu der Erwartung verlängerter Reaktionszeiten unter Medikamenteneinfluss, verkürzte sich die Reaktionszeit tendenziell mit zunehmender Anzahl an Wiederholungen. Dies kann möglicherweise auf Lerneffekte zurückgeführt werden. Ähnliche Ergebnisse ließen sich im Hinblick auf die mittlere motorische Zeit feststellen. Hier wurde die Zeitspanne zwischen dem Verlassen der Ruhetaste und dem Kontakt mit der Drucktaste bei geforderten Reizen gemessen. Abbildung 34 zeigt die entsprechenden Messwerte aller Probanden.

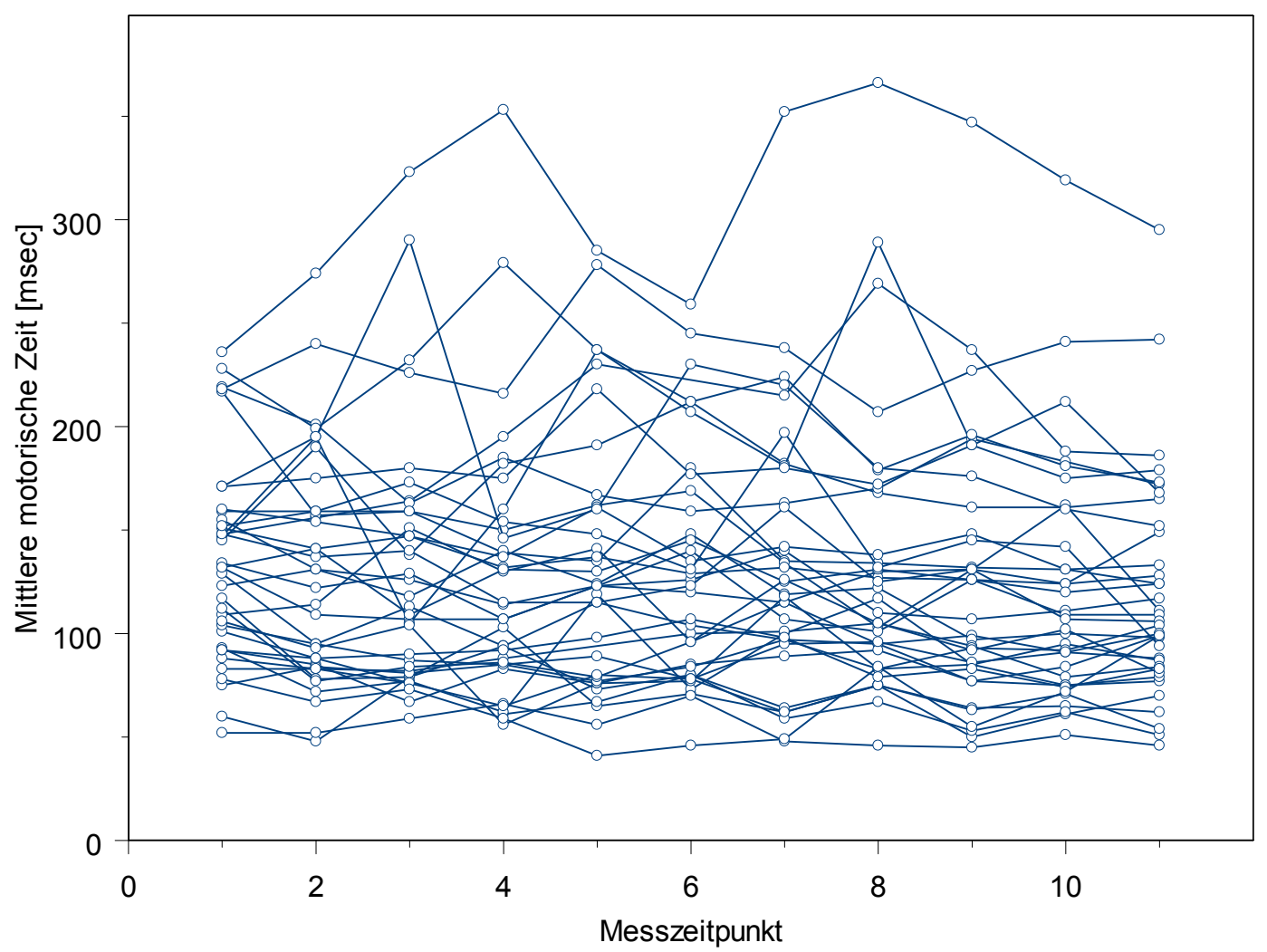

Abb. 34 Mittlere motorische Zeiten aller Probanden; SD, Studientag; TP, Zeitpunkt; Messzeitpunkt 1 = Baseline TP2, Messzeitpunkt 2 = Baseline TP4, Messzeitpunkt $3=$ Baseline TP8, Messzeitpunkt $4=$ Baseline TP11, Messzeitpunkt $5=$ SD1 TP2, Messzeitpunkt $6=$ SD1 TP4, Messzeitpunkt 7 = SD1 TP8, Messzeitpunkt $8=$ SD1 TP11, Messzeitpunkt $9=$ SD2, Messzeitpunkt $10=$ SD3, Messzeitpunkt 11 = SD4; die Medikamentengabe erfolgte 60 min vor Messzeitpunkt 5 .

Unter den mittleren motorischen Zeiten war ebenfalls eine breite Streuung der Messwerte zu erkennen. Es ergab sich auch hier kein medikamentenabhängiger Effekt. Dies galt ebenso für die Unterscheidung nach den CYP2C9-, CYP3A5- und 
OATP1B1-Genotypen. Es wurde kein Unterschied im zeitlichen Auftreten nach der Gabe von Dronabinol deutlich.

\subsubsection{Flimmerfrequenz (FF) und Verschmelzungsfrequenz (VF)}

Die zentralnervöse Aktivierung der Probanden sollte anhand eines Testverfahrens bestimmt werden, bei dem die Studienteilnehmer den Übergang eines flimmernden Lichtpunktes in Gleichlicht (Verschmelzungsfrequenz, VF) und den des Gleichlichtes in Flimmerlicht (Flimmerfrequenz, FF) bestimmen sollten. Sie kennzeichnen den Grad des Aktivierungsniveaus. In Abbildung 35 sind die Flimmerfrequenzen und in Abbildung 36 die Verschmelzungsfrequenzen aller Probanden dargestellt.

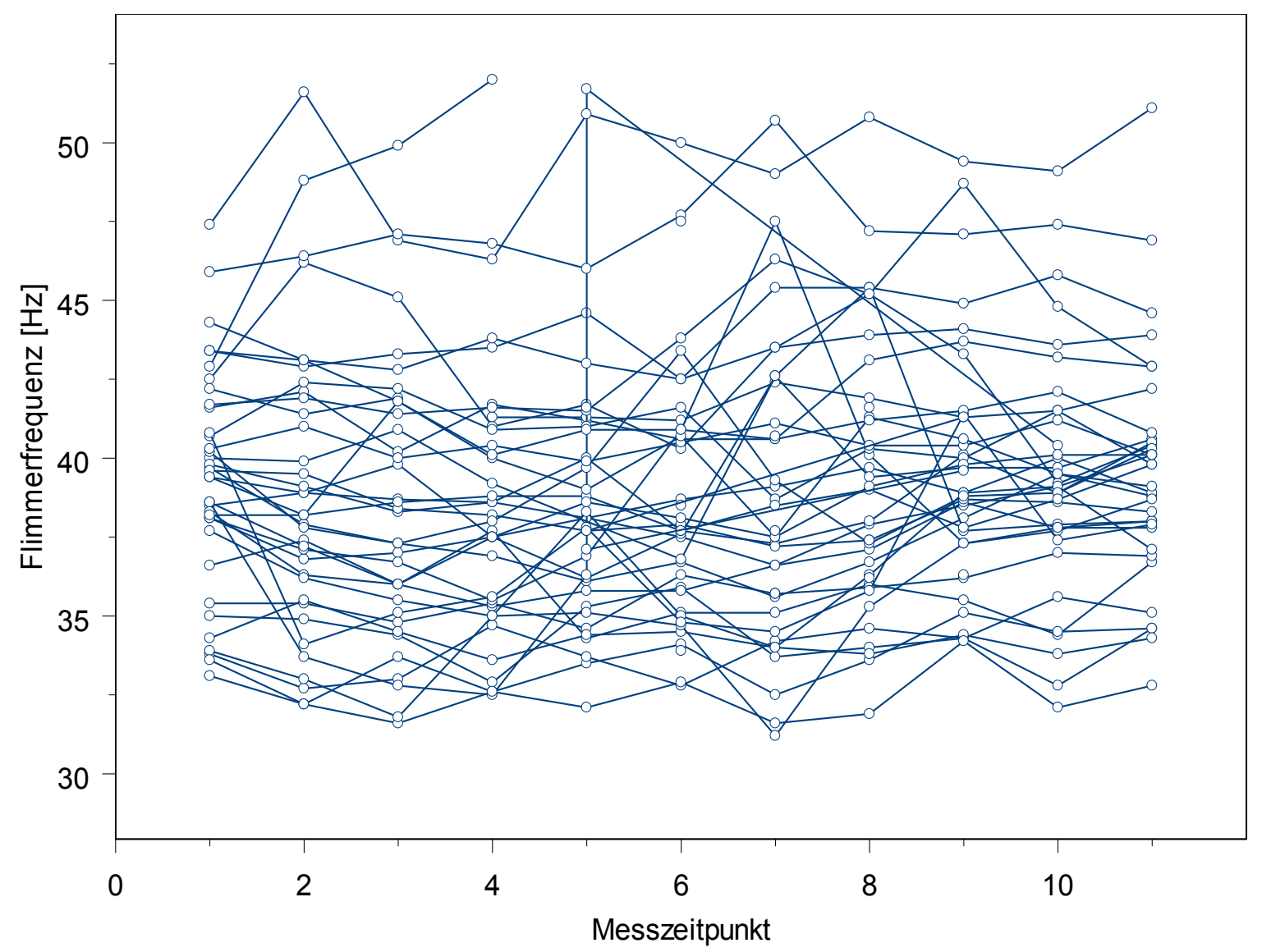

Abb. 35 Flimmerfrequenzen aller Probanden; SD, Studientag; TP, Zeitpunkt; Messzeitpunkt $1=$ Baseline TP2, Messzeitpunkt $2=$ Baseline TP4, Messzeitpunkt $3=$ Baseline TP8, Messzeitpunkt $4=$ Baseline TP11, Messzeitpunkt $5=$ SD1 TP2, Messzeitpunkt $6=$ SD1 TP4, Messzeitpunkt $7=$ SD1 TP8, Messzeitpunkt $8=$ SD1 TP11, Messzeitpunkt $9=$ SD2, Messzeitpunkt $10=$ SD3, Messzeitpunkt 11 = SD4; die Medikamentengabe erfolgte $60 \mathrm{~min}$ vor Messzeitpunkt 5. 


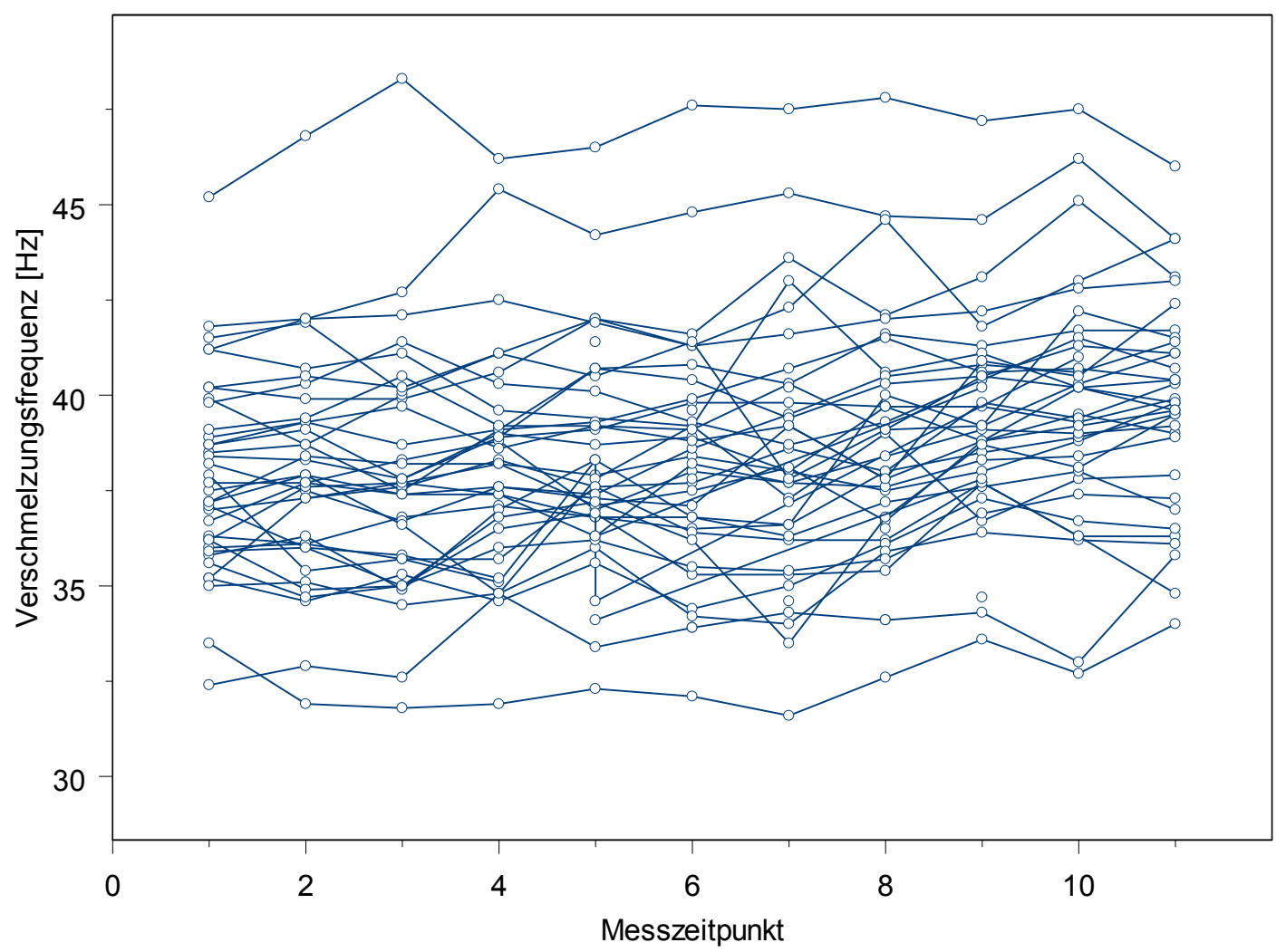

Abb. 36 Verschmelzungsfrequenzen aller Probanden; SD, Studientag; TP, Zeitpunkt; Messzeitpunkt 1 = Baseline TP2, Messzeitpunkt 2 = Baseline TP4, Messzeitpunkt $3=$ Baseline TP8, Messzeitpunkt $4=$ Baseline TP11, Messzeitpunkt $5=$ SD1 TP2, Messzeitpunkt $6=$ SD1 TP4, Messzeitpunkt $7=$ SD1 TP8, Messzeitpunkt $8=$ SD1 TP11, Messzeitpunkt $9=$ SD2, Messzeitpunkt $10=$ SD3, Messzeitpunkt 11 = SD4; die Medikamentengabe war 60 min vor Messzeitpunkt 5.

Sowohl bezüglich der Flimmer-, als auch der Verschmelzungsfrequenz wird deutlich, dass es unter den Probanden zu einer breiten Streuung der Messwerte kam. Ein Effekt der Studienmedikation auf die Ergebnisse dieses Tests ließ sich nicht beobachten. Dies gilt auch nach Stratifizierung hinsichtlich der CYP2C9-, CYP3A5und OATP1B1-Genpolymorphismen.

\subsubsection{Test zur Zeit- und Bewegungsantizipation (ZBA)}

Eine, besonders auch im Hinblick auf die Verkehrspsychologie bedeutsame Fähigkeit ist jene, sich in Bewegungen einfühlen zu können und die Bewegung von Objekten im Raum örtlich und zeitlich richtig einzuschätzen. $\mathrm{Ob}$ sich unter Medikamenteneinfluss diesbezüglich Veränderungen ergaben, wurde mit dem Test zur Zeit- und Bewegungsantizipation geprüft. Auch bei dieser Testform ergab sich eine breite Streuung der Messwerte innerhalb der Probandenkohorte. Es konnte 
sowohl für die Zeit-, als auch für die Bewegungsantizipation kein medikamentenbedingter Effekt nachgewiesen werden. Exemplarisch sind in Abbildung 37 die medianen Abweichungszeiten, aufgetrennt nach CYP2C9Genotypen, dargestellt. Die Abweichungszeit beschreibt, wie genau der zeitliche Verlauf des Testobjekts durch die Studienteilnehmer abgeschätzt wurde. Auch in Hinblick auf die Genpolymorphismen des Enzyms CYP2C9 und die OATP1B1Transportproteine ergaben sich keine Unterschiede in der Zeit- und Bewegungsantizipation.

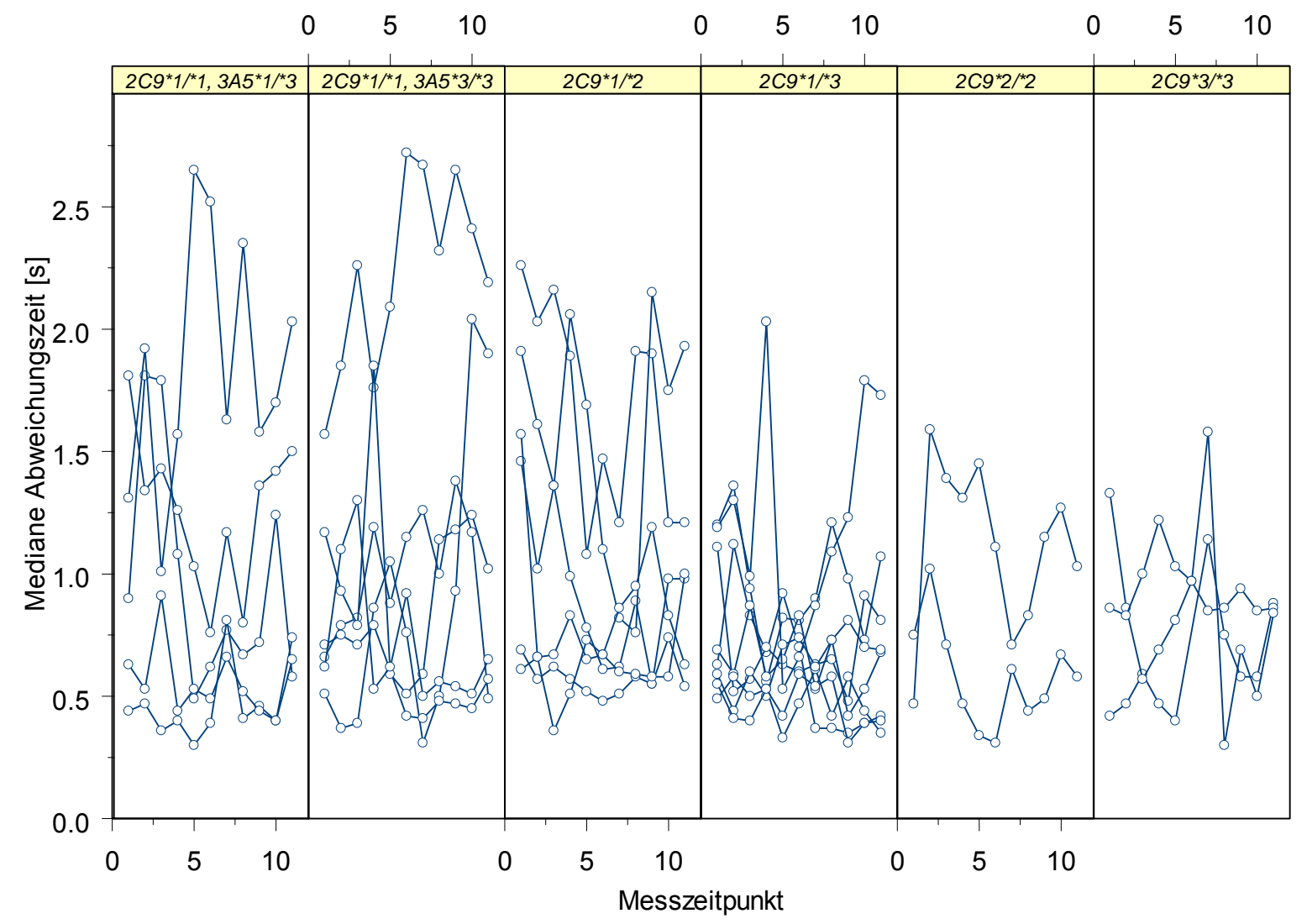

Abb. 37 Mediane Abweichungszeit in Abhängigkeit der CYP2C9-Genpolymorphismen; SD, Studientag; TP, Zeitpunkt; Messzeitpunkt 1 = Baseline TP2, Messzeitpunkt 2 = Baseline TP4, Messzeitpunkt $3=$ Baseline TP8, Messzeitpunkt $4=$ Baseline TP11, Messzeitpunkt $5=$ SD1 TP2, Messzeitpunkt $6=$ SD1 TP4, Messzeitpunkt $7=$ SD1 TP8, Messzeitpunkt $8=$ SD1 TP11, Messzeitpunkt $9=$ SD2, Messzeitpunkt $10=$ SD3, Messzeitpunkt $11=$ SD4; die Medikamentengabe erfolgte $60 \mathrm{~min}$ vor Messzeitpunkt 5. 


\subsubsection{Fortlaufende visuelle Wiedererkennungsaufgabe (FVW)}

Zur Quantifizierung von Gedächtnisleistungen fand die fortlaufende visuelle Wiedererkennungsaufgabe Verwendung. Dabei mussten die Probanden nach der Darbietung bestimmter Items entscheiden, ob diese zum ersten oder zum wiederholten Male gezeigt wurden.

Die Gedächtnisleistung der Studienteilnehmer wurde durch die Studienmedikation nicht beeinflusst. Innerhalb der Probandenkohorte zeigte sich eine breite Streuung der Testergebnisse. Keiner der geprüften molekulargenetischen Faktoren (CYP2C9, CYP3A5, OATP1B1) hatte einen Einfluss auf die Gedächtnisleistung. Exemplarisch sind in Abbildung 38 die Anzahl der richtigen Eingaben (Treffer) aller Probanden in Abhängigkeit vom CYP2C9-Genpolymorphismus dargestellt. Es konnten maximal 100 Treffer erreicht werden.

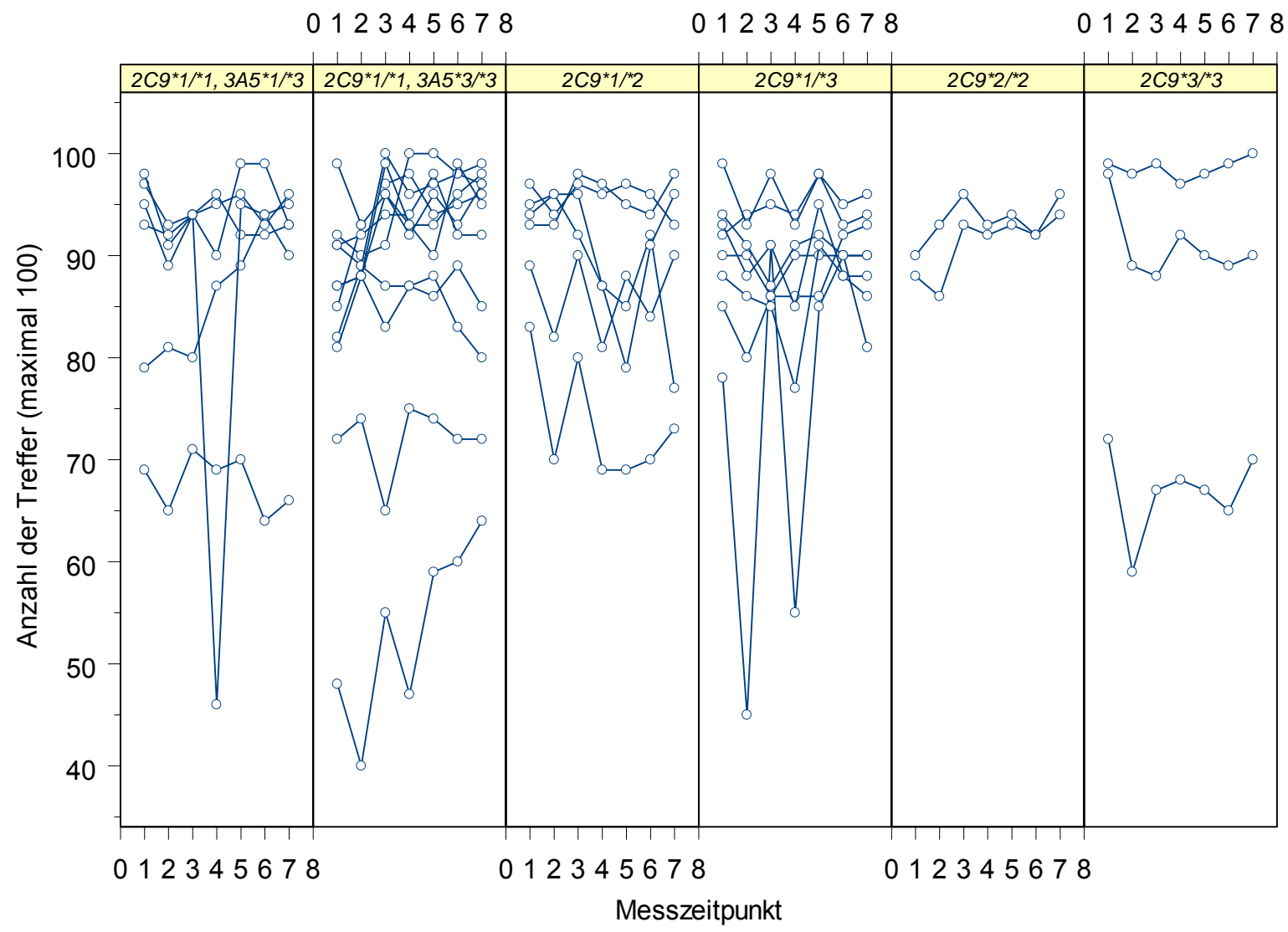

Abb. 38 Anzahl der Treffer bei der fortlaufenden visuellen Wiedererkennungsaufgabe in Abhängigkeit des CYP2C9-Genpolymorphismus; SD, Studientag; TP, Zeitpunkt; Messzeitpunkt 1 = Baseline TP4, Messzeitpunkt $2=$ Baseline TP8, Messzeitpunkt $3=$ SD1 TP4, Messzeitpunkt $4=$ SD1 TP8, Messzeitpunkt $5=$ SD2, Messzeitpunkt $6=$ SD3, Messzeitpunkt $7=$ SD4; Medikamentengabe; die Medikamentengabe erfolgte 150 min vor Messzeitpunkt 3. 


\subsubsection{Erfassung von Stimmungsveränderungen durch den Profile of Mood States (POMS) Test}

Dieser als Eigenschaftswörterliste konstruierte Test sollte Veränderungen der situativen Gefühlszustände der Probanden dokumentieren. Auf einer siebenstufigen Ratingskala wurden Befindlichkeiten hinsichtlich Tatendrang, Müdigkeit, Niedergeschlagenheit und Missmut erfasst. Während der Grad der Niedergeschlagenheit und des Missmuts von der Studienmedikation unbeeinflusst blieb, wurden hinsichtlich der Müdigkeit und des Tatendrangs medikamentenassoziierte Unterschiede in den Testergebnissen deutlich. Der Müdigkeitsgrad nahm unter dem Einfluss des Dronabinols zu. Jedoch ergaben die Zeitpunkte der maximalen Müdigkeit unterschiedliche Ergebnisse. Abbildung 39 stellt die Müdigkeit, nach einer festgelegten Rohwertskala, in Abhängigkeit der CYP2C9-Genotypen dar. Zusätzlich zu den Einzelergebnissen sind Kurven für die gleitenden Mittelwerte dargestellt.

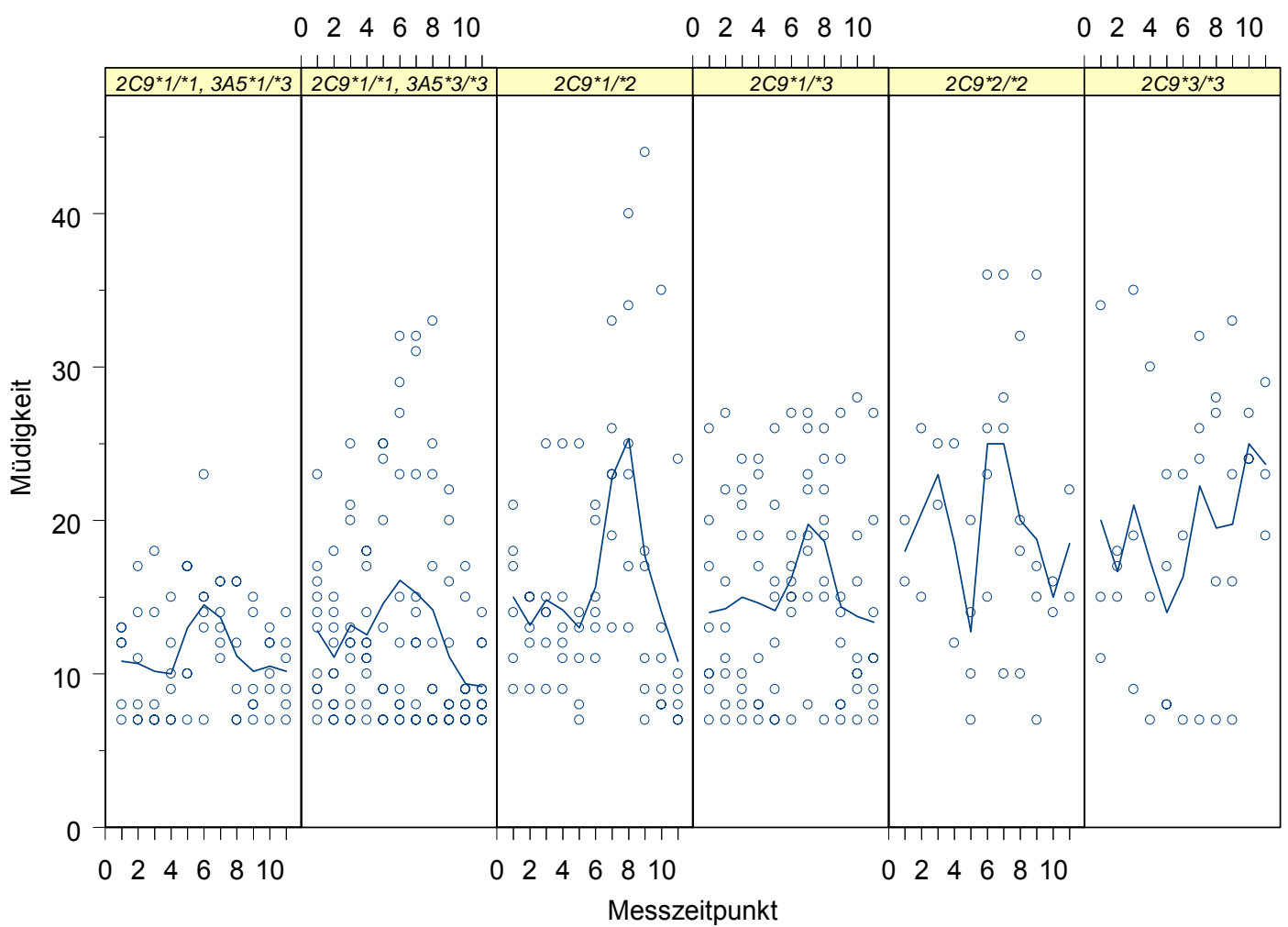

Abb. 39 Müdigkeit der Studienteilnehmer in Abhängigkeit des CYP2C9-Genpolymorphismus; SD, Studientag; TP, Zeitpunkt; Messzeitpunkt 1 = Baseline TP2, Messzeitpunkt 2 = Baseline TP4, Messzeitpunkt $3=$ Baseline TP8, Messzeitpunkt $4=$ Baseline TP11, Messzeitpunkt $5=$ SD1 TP2, Messzeitpunkt $6=$ SD1 TP4, Messzeitpunkt $7=$ SD1 TP8, Messzeitpunkt $8=$ SD1 TP11, Messzeitpunkt $9=$ SD2, Messzeitpunkt $10=$ SD3, Messzeitpunkt $11=$ SD4; die Medikamentengabe erfolgte $60 \mathrm{~min}$ vor Messzeitpunkt 5; die eingezeichnete Linie zeigt einen geglätteten Verlauf der Mittelwerte 
In der Probandengruppe mit der stärksten Fallzahl $\left(\right.$ CYP2C $\left.9{ }^{*} 1 /{ }^{*} 1\right)$ zeigte sich das Maximum der Müdigkeit bei etwa 2.5 Stunden nach Medikamentengabe. Die größten Werte auf der Müdigkeitsskala wiesen die Studienteilnehmer des Genotyps CYP2C9*1/*2 auf. In den Gruppen ${ }^{* 2 / * 2}$ und $* 3 / * 3$ kam es zu größeren Schwankungen der Messwerte.

Ähnliche Erkenntnisse, auch hinsichtlich der CYP2C9-Subgruppen, ergaben sich bei der Betrachtung des Tatendrangs (Abbildung 40). Dieser nahm unter dem Einfluss der Studienmedikation tendenziell ab. In den Gruppen CYP2C9*1/* $1,{ }^{*} 1 /{ }^{*} 2,{ }^{*} 1 /{ }^{*} 3$ und *2/*2 war der geringste Tatendrang um 3 Stunden nach Medikamentengabe zu verzeichnen. In der Gruppe *3/*3 war dies nicht eindeutig zu kennzeichnen.

$\begin{array}{llllll}0 & 2 & 4 & 6 & 8 & 10\end{array}$ $\begin{array}{llllll}0 & 2 & 4 & 6 & 8 & 10\end{array}$ 0246810

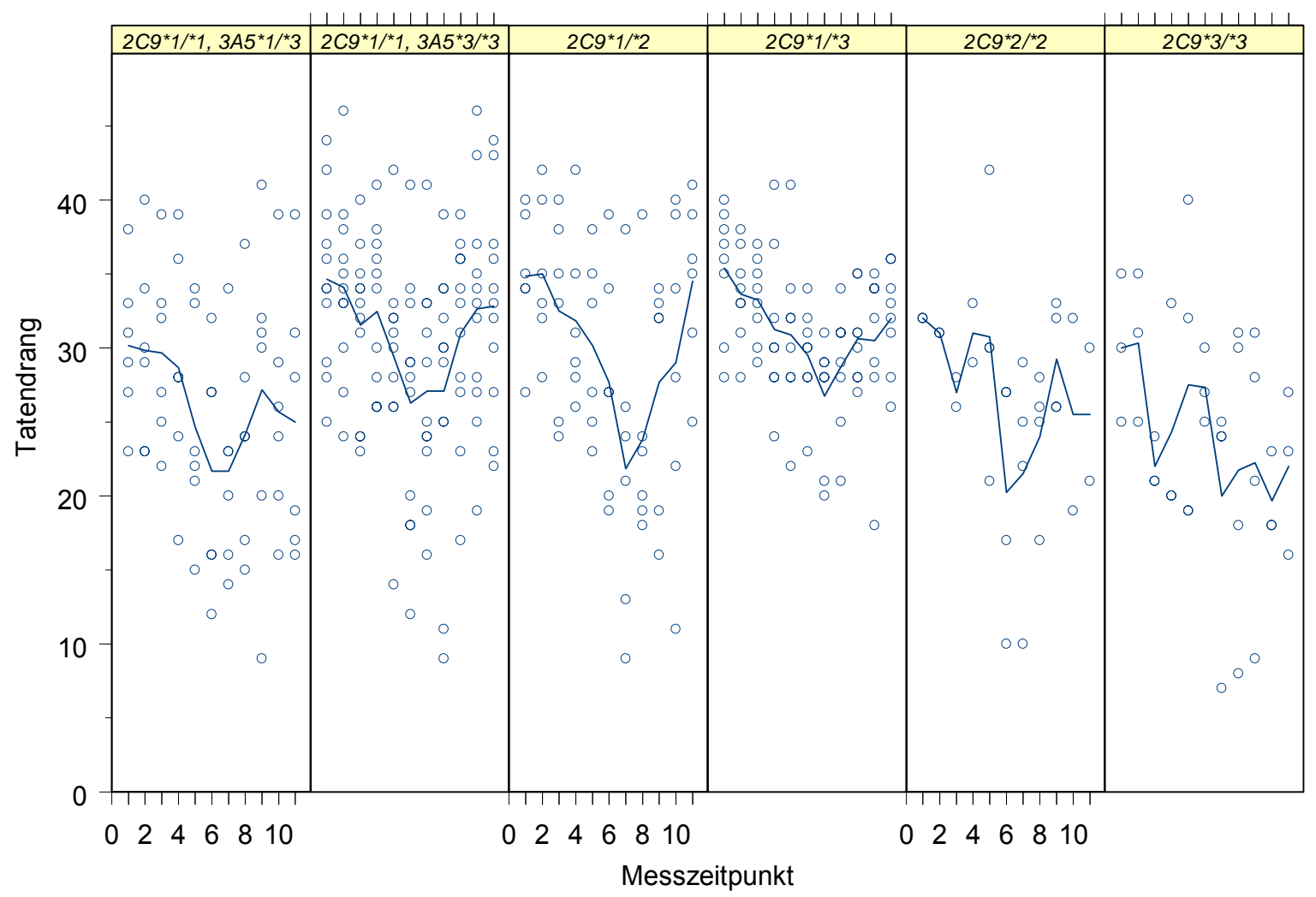

Abb. 40 Tatendrang aller Versuchsteilnehmer in Abhängigkeit der CYP2C9-Genpolymorphismen; SD, Studientag; TP, Zeitpunkt; Messzeitpunkt 1 = Baseline TP2, Messzeitpunkt 2 = Baseline TP4, Messzeitpunkt $3=$ Baseline TP8, Messzeitpunkt $4=$ Baseline TP11, Messzeitpunkt $5=$ SD1 TP2, Messzeitpunkt $6=$ SD1 TP4, Messzeitpunkt $7=$ SD1 TP8, Messzeitpunkt $8=$ SD1 TP11, Messzeitpunkt $9=$ SD2, Messzeitpunkt $10=$ SD3, Messzeitpunkt $11=$ SD4; die Medikamentengabe war 60 min vor Messzeitpunkt 5; die eingezeichnete Linie zeigt einen geglätteten Verlauf der Mittelwerte 
Als ein weiterer Parameter des POMS-Tests wurde die Bearbeitungszeit erfasst. Sowohl die Einzelwerte, als auch die Kurven der Mittelwerte sind in Abbildung 41 in Abhängigkeit der CYP2C9-Genotypen dargestellt. Es ist zu erkennen, dass die Bearbeitungszeit unter Medikamenteneinfluss tendenziell zunahm. Eindeutige Trends ließen sich jedoch, auch aufgrund der großen Streuung der Werte, nicht zuordnen.

Die CYP3A5- und OATP1B1-Genpolymorphismen hatten keinen Einfluss auf die Ergebnisse des POMS-Testes.

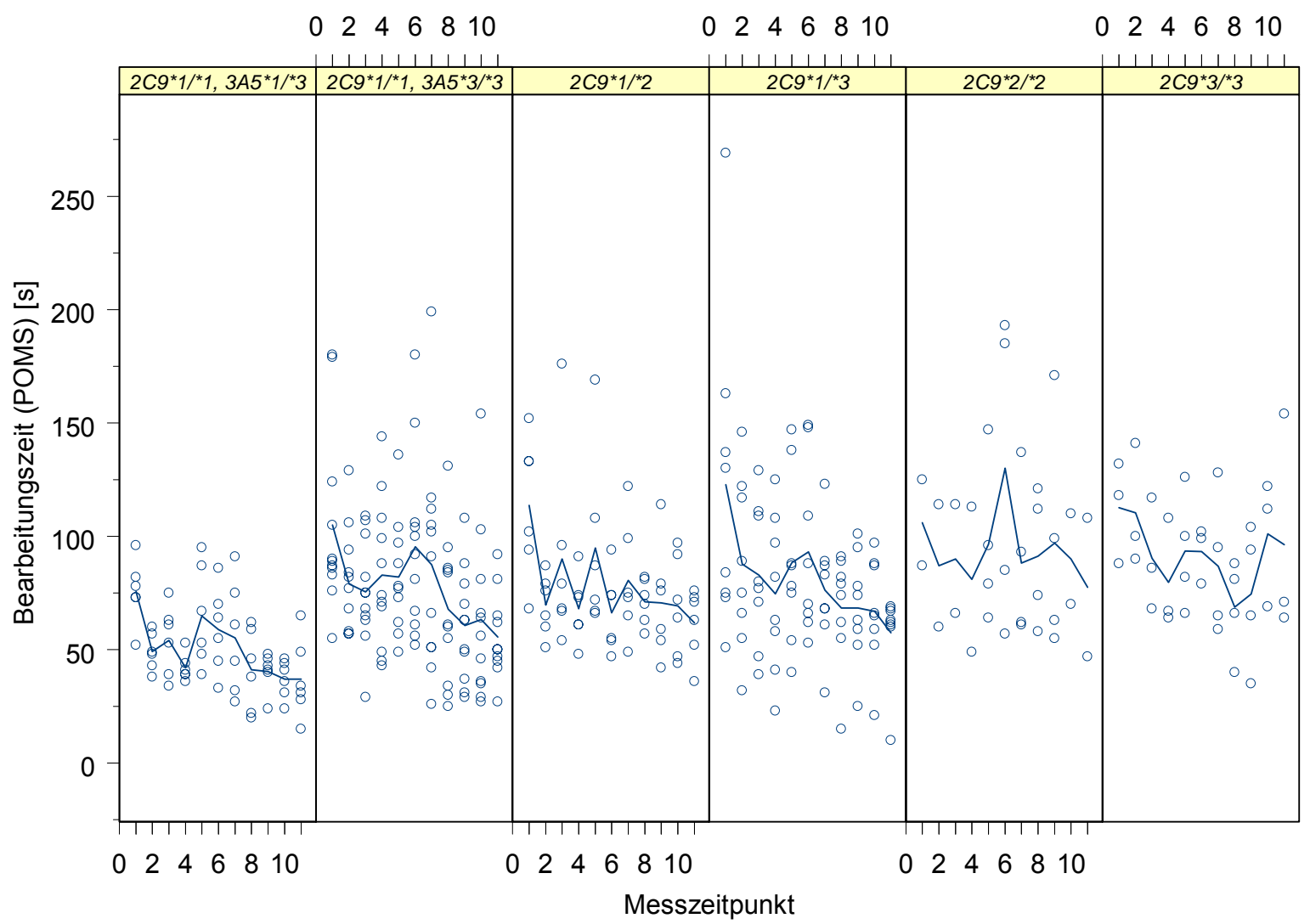

Abb. 41 Bearbeitungszeit des POMS-Tests aller Versuchsteilnehmer in Abhängigkeit der CYP2C9Genpolymorphismen; SD, Studientag; TP, Zeitpunkt; Messzeitpunkt 1 = Baseline TP2, Messzeitpunkt 2 = Baseline TP4, Messzeitpunkt $3=$ Baseline TP8, Messzeitpunkt $4=$ Baseline TP11, Messzeitpunkt $5=$ SD1 TP2, Messzeitpunkt $6=$ SD1 TP4, Messzeitpunkt $7=$ SD1 TP8, Messzeitpunkt $8=$ SD1 TP11, Messzeitpunkt $9=$ SD2, Messzeitpunkt $10=$ SD3, Messzeitpunkt $11=$ SD4; die Medikamentengabe war 60 min vor Messzeitpunkt 5; die eingezeichnete Linie zeigt einen geglätteten Verlauf der Mittelwerte 


\subsection{Einfluss von THC und dem CYP2C9-Genotyp auf den Geruchssinn}

Die Wahrnehmung von Gerüchen kann unter dem Einfluss von THC verändert sein. Um dies zu untersuchen, wurde ein Riechtest, einmal am Baseline- und einmal am Verumtag durchgeführt. Dabei wurde die Riechschwelle der Probanden für die Geruchsqualitäten Schokolade, Ananas, Vanille, Schinken und Rose bestimmt. Als wesentliche mögliche Einflussfaktoren auf das Riechvermögen wurden die Verumgabe, der CYP2C9-Genotyp und das Geschlecht im Rahmen einer univariaten Varianzanalyse ausgewertet (Tabelle 17).

Tab. 17 Untersuchte Geruchsstoffe hinsichtlich möglicher Einflussfaktoren

\begin{tabular}{cccccc}
\hline Faktor & \multicolumn{5}{c}{ Geruchsstoff } \\
\hline THC & Schokolade & Ananas & Vanille & Schinken & Rose \\
CYP2C9- & n.s. & $p=0.036$ & n.s. & n.s. & n.s. \\
Genotyp & n.s. & $p=0.006$ & $p=0.013$ & n.s. & $p=0.016$ \\
Geschlecht & n.s. & n.s. & n.s. & n.s. & n.s. \\
\hline
\end{tabular}

p-Werte entsprechend der univariaten Varianzanalyse; n.s., nicht signifikant

Einzig die Geruchsqualität von Ananas wurde signifikant durch die Verumgabe beeinflusst. Die Mittelwerte der Riechschwelle waren 15.0 am Baselinetag und 13.7 am Verumtag. Die Ergebnisse aller weiteren Geruchsrichtungen unterschieden sich vom Baselinetag zum Verumtag (keine Medikamentengabe vs. Medikamentengabe) nicht signifikant. Einen gewissen Einfluss hatten die CYP2C9-Genotypen. Für Ananas, Vanille und Rose ergaben sich signifikante Unterschiede. Schokolade war mit $p=0.052$ formal nicht mehr signifikant, der $p$-Wert könnte jedoch auf einen entsprechenden Trend hinweisen. Ein geschlechtsspezifischer Unterschied des Riechvermögens konnte nicht gezeigt werden. Für den Geruchsstoff Ananas ist in Abbildung 42 die Riechschwelle in Abhängigkeit vom CYP2C9-Genotyp und von der THC-Gabe dargestellt. Während man sehr einheitlich eine Verschlechterung des Geruchssinns unter THC sieht, sind zwar Unterschiede zwischen den CYP2C9-Genotypen zu erkennen, aber keine einheitliche Korrelation zwischen genetisch vorausgesagter CYP2C9-Aktivität und dem Geruchssinn. 


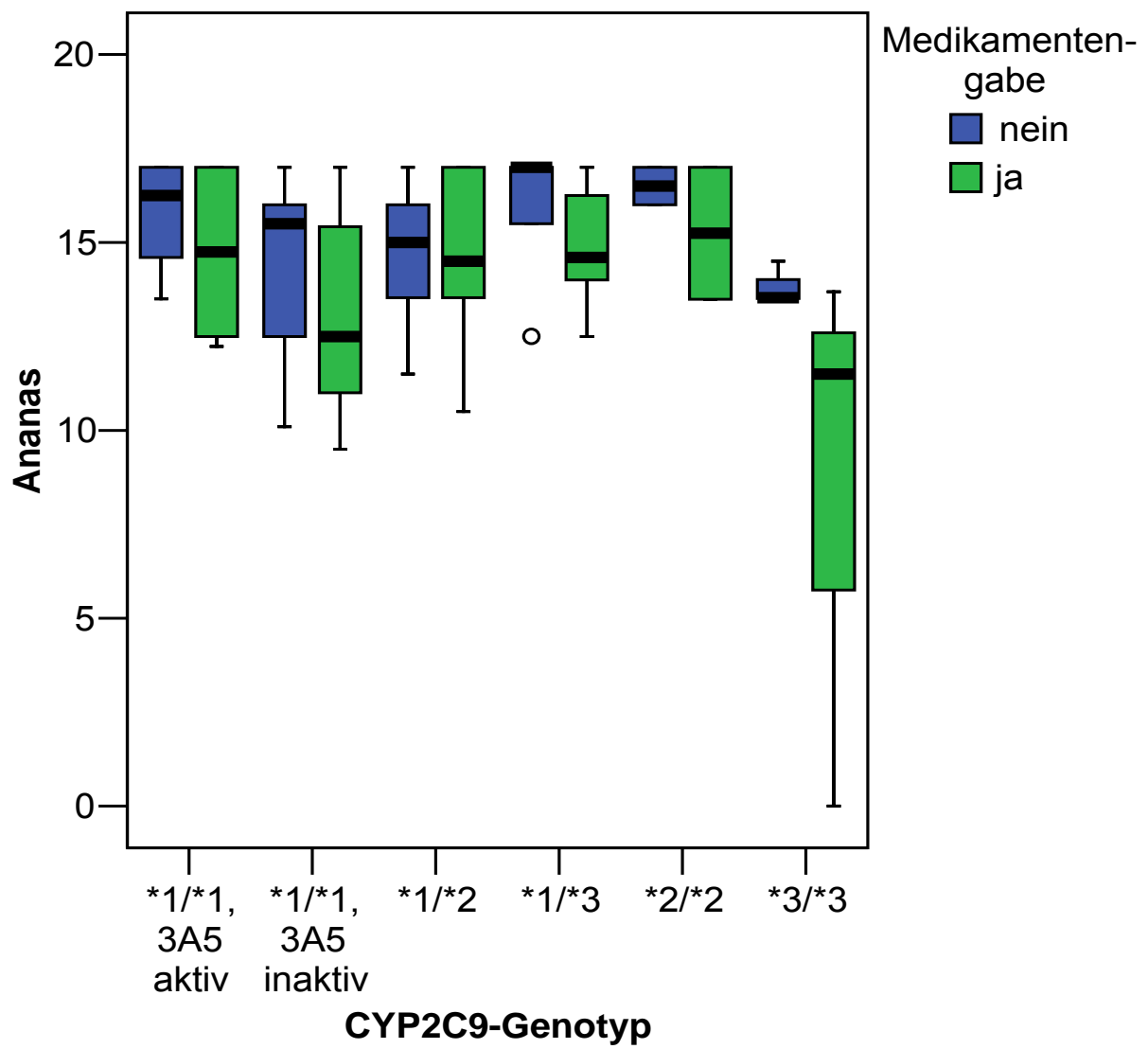

Abb. 42 Riechschwelle von Ananas, bezogen auf Baseline- und Verumtag, in Abhängigkeit der CYP2C9-Genotypen. Auf der Ordinate ist die Verdünnungsstufe dargestellt, in der das nach Ananas riechende Parfüm noch wahrgenommen werden konnte. 


\section{Diskussion}

Die Hauptfragestellung der vorliegenden klinischen Studie war, ob und inwiefern die Pharmakokinetik von THC und seinen Metaboliten durch genetische Polymorphismen der Enzyme CYP2C9 und CYP3A5 beeinflusst wird. Es konnte gezeigt werden, dass es in Abhängigkeit des Genotyps CYP2C9*3 zu statistisch signifikanten Unterschieden der Pharmakokinetik von THC und $\mathrm{COOH}-\mathrm{THC}$ kommt. Bezogen auf das Enzym CYP3A5 konnten keine signifikanten pharmakokinetischen Differenzen herausgestellt werden. Dagegen konnte zusätzlich das Geschlecht als signifikanter Faktor determiniert werden. Abbildung 43 gibt nochmals einen zusammenfassenden Überblick bezüglich der Pharmakokinetik des THC und seiner Metaboliten. Dargestellt ist hier die AUC in Abhängigkeit der Anzahl der CYP2C9*3-Allele. Die Abbildung zeigt, dass die AUC von THC und $\mathrm{COOH}-\mathrm{THC}$ statistisch signifikant von der Zahl an CYP2C9*3-Allelen beeinflusst wurde.

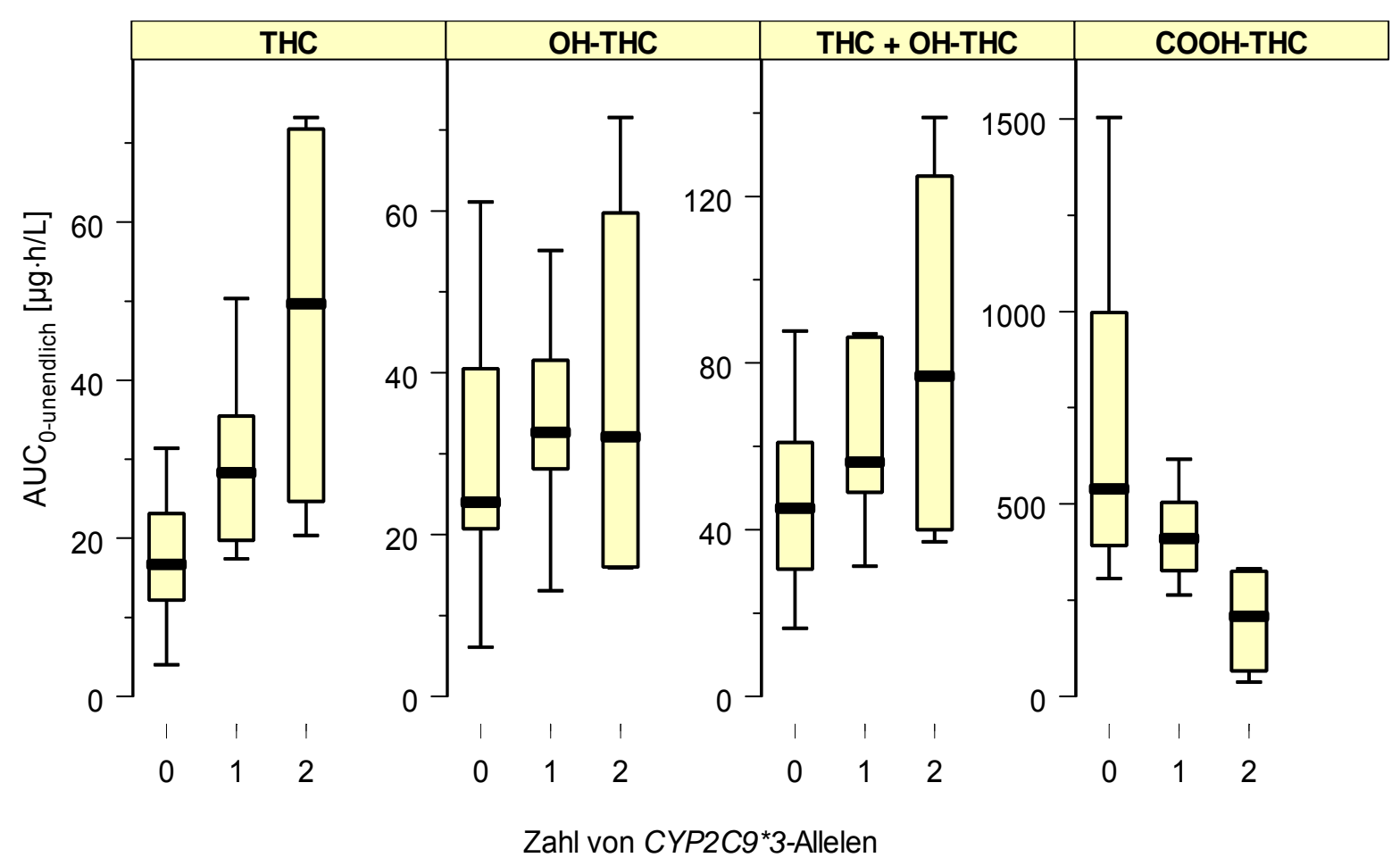

Abb. 43 AUCs von THC, OH-THC, der Summe aus THC und OH-THC sowie $\mathrm{COOH}-\mathrm{THC}$ in Abhängigkeit der Zahl an CYP2C9*3-Allelen 


\subsection{Pharmakokinetik im Vergleich zur Literatur}

\subsubsection{Pharmakokinetik von THC, OH-THC und COOH-THC im Blutplasma}

Bis zum heutigen Zeitpunkt wurde die Pharmakokinetik von THC und seinen Derivaten in einigen in-vitro- und in-vivo-Studien untersucht. Die Applikationsformen waren dabei ebenso vielfältig (Inhalation, orale Einnahme, intravenöse Gabe) wie die Dosierungen. Im Folgenden soll ein Vergleich unserer pharmakokinetischen Daten mit denen vorangegangener Studien erfolgen.

Bezüglich der maximalen Plasmakonzentration $\left(\mathrm{C}_{\max }\right)$ von THC ergaben sich in unserer Studie je nach Allelkonstellation des CYP2C9-Enzyms mittlere Werte im Bereich von 2.7 bis $6.3 \mu \mathrm{g} / \mathrm{L}$. Für den Primärmetaboliten $\mathrm{OH}-\mathrm{THC}$ lagen diese zwischen 2.8 und $6.5 \mu \mathrm{g} / \mathrm{L}$. Folglich waren die Konzentrationen des THC also annähernd gleich mit denen des OH-THC. Zu diesem Ergebnis kamen auch Goodwin et al. (2006). Sie verabreichten dreimal täglich Dronabinol mit der Tagesdosis von $7.5 \mathrm{mg}$ über fünf Tage. Die maximale Plasmakonzentration überschritt dabei zu keiner Zeit $6.1 \mu \mathrm{g} / \mathrm{L}$. Wall und Perez-Reyes (1981) erhielten bei einer oralen Dosierung von 20 mg THC ebenso annähernd ähnliche Plasmaspiegel von THC und OH-THC. Die mittleren Werte lagen zwischen 6 und $8 \mu \mathrm{g} / \mathrm{L}$. Unter Berücksichtigung einer zur Dosis proportionalen Pharmakokinetik entspricht dies den Werten unserer Studie (Kapitel 3.2).

Die mittleren Zeitpunkte der maximalen Blutkonzentrationen $\left(t_{\max }\right)$ des THC waren in unserer Studie mit 2 bis 4 Stunden annähernd gleich derer von $\mathrm{OH}-\mathrm{THC}$ und $\mathrm{COOH}-$ THC. Dies entspricht den Ergebnissen von Wall und Perez-Reyes (1981), in deren Untersuchung sich maximale Blutkonzentrationen von THC und $\mathrm{OH}-\mathrm{THC}$ nach 2 bis 3 Stunden zeigten. THC unterliegt einem substantiellen First-Pass-Effekt (McGilveray 2005). Dies wird dadurch weiter erhärtet, dass die Zeitpunkte der maximalen Plasmakonzentration $\left(t_{\max }\right)$ des $\mathrm{COOH}-\mathrm{THC}$ sehr nahe bei denen des THC lagen.

Ausgehend von einem Zwei-Kompartiment-Modell, das für den THC-Metabolismus bereits im Vorfeld beschrieben wurde (Wall et al. 1983, Nadulski et al. 2005) und auch in unserer Auswertung Anwendung fand (Kapitel 3.3), lagen die terminalen Halbwertszeiten von THC in der vorliegenden Studie, in Abhängigkeit der 
Genpolymorphismen, im Mittel zwischen 7 und 22 Stunden. Die Untersuchung von Wall et al. (1983) ergab dagegen mittlere Halbwertszeiten um 25 Stunden.

Bezüglich der Unterschiede in der Pharmakokinetik von THC in Abhängigkeit des Geschlechts sind in der Vergangenheit kontroverse Ergebnisse erzielt worden. Die Arbeitsgruppe Wall et al. (1983) konnte keine geschlechtsspezifischen Unterschiede feststellen. Nadulski et al. (2005) verabreichten über vier Wochen einmal wöchentlich $2.5 \mathrm{mg}$ THC. Sie kamen zu dem Ergebnis, dass hinsichtlich aller Verbindungen (THC, OH-THC, COOH-THC) sowohl die Werte für die AUC, als auch die der maximalen Plasmakonzentration $\left(\mathrm{C}_{\max }\right)$ bei Frauen signifikant höher waren als bei Männern. Lediglich für die AUC von $\mathrm{COOH}-\mathrm{THC}$ konnte keine Signifikanz, jedoch ein entsprechender Trend nachgewiesen werden. Die Arbeitsgruppe Nadulski et al. (2005) erklärt diese Differenzen unter den Geschlechtern dadurch, dass Frauen ein kleineres Verteilungsvolumen für THC haben. Dies führen sie nicht auf das geringere Körpergewicht zurück, sondern auf geschlechtsspezifische Unterschiede im Verteilungsvolumen während des THC-Metabolismus. Auch die Daten unserer Studie ergaben geschlechtsabhängige Unterschiede in der Pharmakokinetik von THC. Die medianen maximalen Blutkonzentrationen von THC lagen bei den Frauen signifikant höher als bei den Männern. Bezüglich der Metaboliten $\mathrm{OH}-\mathrm{THC}$ und $\mathrm{COOH}-\mathrm{THC}$ konnte nur ein entsprechender Trend zu höheren Werten bei den Frauen nachgewiesen werden. Im Hinblick auf die orale Clearance fanden sich die medianen Werte der männlichen stets über denen der weiblichen Probanden (Kapitel 3.6). Aufgrund der Tatsache, dass der Unterschied zwischen Männern und Frauen sowohl hinsichtlich der Ausgangssubstanz THC, als auch in Bezug auf die Metaboliten erkennbar war, ist nicht davon auszugehen, dass hier Differenzen in der Enzymaktivität ursächlich sind. Vielmehr ist anzunehmen, dass die verschiedenen Verteilungsvolumina und damit indirekt das Ausmaß des Körperfettanteils eine bedeutende Rolle spielen. Im Gegensatz zu unserer Studie ergab sich in einer Untersuchung von Goodwin et al. (2006) eine Korrelation zwischen BMI und der maximalen Plasmakonzentration $\left(\mathrm{C}_{\max }\right)$ für $\mathrm{THC}$ und $\mathrm{OH}-\mathrm{THC}$.

Bezüglich der AUC nach oraler Applikation von THC gibt es wenige Daten in der Literatur. In unserer Studie lagen die Mediane für THC zwischen $16.1 \mu \mathrm{g} \cdot \mathrm{h} / \mathrm{L}$ für CYP2C9*1/*1 und $49.7 \mu \mathrm{g} \cdot \mathrm{h} / \mathrm{L}$ für CYP2C9*3/*3. Dies deckt sich mit der Studie von Ohlsson et al. (1980), in der eine durchschnittliche AUC von $17 \mu \mathrm{g} \cdot \mathrm{h} / \mathrm{L}$ für THC angegeben wird. Da über zwei Drittel der europäischen Bevölkerung den Genotyp 
CYP2C9*1/*1 tragen und nur etwa $0.4 \%$ homozygot für das Allel CYP2C9*3 sind, muss für den Vergleich mit den Daten von der Arbeitsgruppe um Ohlsson also eher der Median von $16.1 \mu \mathrm{g} \cdot \mathrm{h} / \mathrm{L}$ für die AUC von THC herangezogen werden.

Unter der Annahme einer vom Genotyp unabhängigen Bioverfügbarkeit $(F)$ von 6\% (Grotenhermen 2003) ergeben sich aus den Medianen der oralen Clearances (Cl/F) von $15.5\left({ }^{*} 1 /{ }^{*} 1\right), 7.9\left({ }^{*} 1 /{ }^{*}\right)$ und $6.1 \mathrm{~L} / \mathrm{min}\left({ }^{*} 3 /{ }^{*}\right)$ mediane systemische Clearances von $0.93\left({ }^{*} 1 /{ }^{*} 1\right), 0.47\left({ }^{*} 1 /{ }^{*} 3\right), 0.37 \mathrm{~L} / \mathrm{min}\left({ }^{*} 3 /{ }^{*} 3\right)$. Grotenhermen (2003) kommt in seinem Review zu dem Ergebnis, dass die systemische Clearance von THC im Bereich von 0.7 bis $1.0 \mathrm{~L} / \mathrm{min}$ liegt. Auch im Hinblick auf diese Daten ist zu berücksichtigen, dass die Genpolymorphismen des Enzyms CYP2C9 bei der Erhebung dieser Daten nicht mit einbezogen worden sind und auch die Fallzahlen, mit maximal sechs Probanden, deutlich unter denen unserer Studie lagen. Daher lässt sich auch an dieser Stelle nur die Clearance des mit Abstand am häufigsten vorkommenden Genotyps CYP2C9*1/*1, die in unserer Studie $0.93 \mathrm{~L} / \mathrm{min}$ betrug, mit der von Grotenhermen (2003), die wie bereits erwähnt, zwischen 0.7 und 1.0 L/min lag, vergleichen. Unter Berücksichtigung dieses Gesichtspunktes entsprechen die Daten einander.

Nichtsdestotrotz stimmen die Parameter unserer Studie nicht vollständig mit dem überein, was in früheren Studien beschrieben wurde. Dies wäre beispielsweise der Fall, wenn wir für den Vergleich der systemischen Clearances von einer Bioverfügbarkeit oberhalb von sechs Prozent ausgegangen wären (McGilveray 2005). Bei der Bewertung dieser Unterschiede muss bedacht werden, dass die Plasmakonzentrationen von THC und seinen Metaboliten von vielen Faktoren beeinflusst werden. Neben den arzneimittelmetabolisierenden Enzymen zählen dazu auch das Ausmaß der gastrointestinalen Absorption, welches für THC nach McGilveray (2005) sehr variabel ist, der First-Pass-Metabolismus, das Ausmaß der biliären Exkretion, die Verteilung des Arzneistoffes in das Gewebe, die Rückverteilung aus dem Gewebe, die Clearance und viele weitere Faktoren. Nicht zuletzt muss berücksichtigt werden, dass es lange Zeit keine standardisierten pharmazeutischen Präparationen von THC gab und insbesondere die Bioverfügbarkeit sehr von der Galenik abhängt. Begleitsubstanzen in der oralen Applikationsform können die Bioverfügbarkeit erheblich beeinflussen. Wie bereits erwähnt, gibt es nur begrenzte Daten vorangegangener Studien zur Pharmakokinetik von Delta-9-THC. Diskrepanzen zwischen den Daten der vorliegenden Studie und 
denen früherer Untersuchungen lassen sich auch dadurch erklären, dass in der Vergangenheit noch keine in-vivo-Studie mit der einmaligen Dosierung von $15 \mathrm{mg}$ Dronabinol erfolgt ist. Unsere Ergebnisse lassen sich daher nur bedingt mit denen anderer Studien vergleichen, in denen beispielsweise Hanföl oder Schokoladengebäck eingenommen oder Marihuana geraucht wurde. Die Pharmakokinetik von THC nach Inhalation und oraler Gabe lässt sich kaum gegenüberstellen. Die oralen Dosierungen früherer Untersuchungen lagen im Bereich von 2.5 bis $30 \mathrm{mg}$ und wurden einmalig, aber auch bis zu dreimal täglich über mehrere Wochen verabreicht. Des Weiteren mussten die Probanden unserer Studie im Gegensatz zu denen einiger anderer Untersuchungen eine interaktionsarme Diät einhalten und durften keine oralen Kontrazeptiva einnehmen. Im Hinblick auf die Anzahl an Versuchsteilnehmern kann unsere Studie mit 43 Probanden die deutlich größte Fallzahl der bisherigen Untersuchungen vorweisen.

\subsubsection{Pharmakokinetik von COOH-THC im Urin}

Bezüglich der Ausscheidung von THC und seiner Metaboliten wurde der Urin der Probanden untersucht. Die renale Elimination spielte eine untergeordnete Rolle. Auch Dietz et al. (2007) kamen zu dem Ergebnis, dass die biliäre Elimination mit anschließender Ausscheidung über den Faeces für THC am bedeutendsten ist. Die Analyse in unserer Studie ergab insgesamt sehr geringe Mengen von THC-Ausscheidungsprodukten im Urin. Als einzige Substanz wurde von uns das glukuronidierte $\mathrm{COOH}-\mathrm{THC}$ detektiert. Dies entspricht den Ergebnissen früherer Studien (Wall et al. 1983; Dietz et al. 2007). Die mediane kumulative Ausscheidung von $\mathrm{COOH}-\mathrm{THC}$ über 24 Stunden der gesamten Probandenkohorte betrug in unserer Studie $109.4 \mu \mathrm{g}$. Minimum und Maximum lagen bei 6.1 - 410.8 $\mu \mathrm{g}$ (Kapitel 3.7.1). In einer Studie von 2004 wurden in einem Multiple-Dose-Verfahren 7 Probanden 4 verschiedene orale Dosierungen von THC verabreicht $(0.39,0.47,7.5$ und $14.8 \mathrm{mg}$ THC/Tag; über 5 Tage). Es traten dabei keine statistisch signifikanten Unterschiede zwischen den verschiedenen Dosierungen auf. Untersucht wurden die Mittelwerte der maximalen Ausscheidungsrate $(0.9$ - $16.3 \mu \mathrm{g} / \mathrm{h})$, die dazugehörigen Zeitpunkte (67.4 - 94.9 h) und die terminale Halbwertszeit (44.2 - 64.0 h). Bei einer mit unserer Studie gut vergleichbaren Tagesdosis von $14.8 \mathrm{mg}$ THC wurden innerhalb von 
14 Tagen lediglich $0.6 \%+/-0.5 \%$ als glukuronidiertes $\mathrm{COOH}-\mathrm{THC}$ im Urin detektiert (Gustafson et al. 2004). In unserer Studie betrug dieser Anteil innerhalb von 24 Stunden nach Verabreichung von $15 \mathrm{mg}$ Dronabinol etwa 0.73\%. Die maximale Ausscheidungsrate pro Stunde war $34.2 \mu \mathrm{g} / \mathrm{h}$. In einer früheren Arbeit wurden bis zum Zeitpunkt von 72 Stunden nach der Medikamentengabe etwa $13-16 \%$ der totalen oralen THC-Dosis im Urin gefunden (Wall et al. 1983). Hier handelt es sich jedoch um eine Studie mit radioaktiv markiertem THC, wodurch die Summe aller aus THC entstandenen Metaboliten quantifiziert wurde.

Die Diskrepanzen der Ergebnisse verglichen mit denen anderer Studien ergeben sich vermutlich auch daraus, dass die Menge des COOH-THCs, die im Urin erscheint, höchst variabel ist (Dietz et al. 2007). Des Weiteren wurde der Urin in unserer Studie nur über 24 Stunden gesammelt, während andere Studien die Urinausscheidung für mindestens 72 Stunden verfolgten.

In unserer Studie gab es keine geschlechtsspezifischen Unterschiede hinsichtlich der Ausscheidung von COOH-THC. Wall et al. (1983) kamen ebenfalls zu diesem Ergebnis.

\subsection{Pharmakokinetik und Biotransformation durch CYP2C9}

\subsubsection{Einfluss des CYP2C9-Genpolymorphismus auf THC und seine Metaboliten im Plasma}

In vorangegangenen in-vitro-Untersuchungen konnte bereits die Beteiligung der CYP2C9-Enzyme an der Biotransformation von THC aufgezeigt werden (Bland et al. 2005). Die vorliegende Studie sollte untersuchen, ob und in welchem Ausmaß sich die Genvarianten CYP2C9*2 und CYP2C9*3 auf den THCMetabolismus beim Menschen auswirken.

In biochemischen Analysen wird typischerweise eine Substanz (hier THC) entweder mit so genannten Lebermikrosomen (endoplasmatisches Retikulum, gereinigt durch Ultrazentrifugation) oder mit gentechnologisch (rekombinant) exprimierten Cytochrom-P450-Enzymen inkubiert und der Metabolismus gemessen. Charakteristische Kenngrößen bei diesen Experimenten sind die maximale Metaboliten-Bildungsgeschwindigkeit $\left(\mathrm{V}_{\max }\right)$ und die Substratkonzentration bei halbmaximaler Metabolisierungsgeschwindigkeit $\left(\mathrm{K}_{\mathrm{M}}\right)$. In vitro beträgt die intrinsische 
Clearance $\left(\mathrm{V}_{\max } / \mathrm{K}_{\mathrm{M}}\right)$ für das Allel $\star_{3} \mathrm{im}$ Vergleich zur Variante $\star_{1}$ nur etwa $30 \%$ (Bland et al. 2005). Dies entsprach den Daten unserer Studie, die eine reduzierte orale Clearance in Trägern der Variante $C Y P 2 C 9 * 3 / * 3$ gegenüber denen des Genotyps CYP2C9*1/*1 zeigte (Kapitel 3.2.2). Die Plasmakonzentrationen des primären THC-Metaboliten $\mathrm{OH}-\mathrm{THC}$ ergaben im Vergleich der Varianten CYP2C9*2 und CYP2C9*3 mit dem Genotyp CYP2C9*1/*1 keine erniedrigten Werte. Diese Beobachtung lässt sich am ehesten anhand der Annahme erklären, dass die CYP2C9-Enzyme im Metabolismus von THC zu OH-THC lediglich eine untergeordnete Rolle spielen. Dahingegen scheinen sie einen wesentlichen Teil bei der Umwandlung von $\mathrm{OH}-\mathrm{THC}$ zu COOH-THC beizutragen (Watanabe et al. 2007). Das steht im Gegensatz zu den allgemein verbreiteten Darstellungen, nach denen die Umwandlung von $\mathrm{OH}-\mathrm{THC}$ zu $\mathrm{COOH}-\mathrm{THC}$ eher durch die AlkoholDehydrogenase oder die Aldehyd-Dehydrogenase katalysiert wird (Abbildung 3).

Im Gegensatz zu dem Allel CYP2C9*3 ließen sich innerhalb der Gruppe der Genotypen, die durch das Allel CYP2C9*2 definiert wurden, keine Unterschiede zwischen den Plasmakonzentrationen von THC, OH-THC und $\mathrm{COOH}-\mathrm{THC}$ herausstellen. Die Studienlage bezüglich der Bedeutung der CYP2C9*2-Variante für den Arzneimittelmetabolismus ist nicht einheitlich. Es konnte einerseits bereits gezeigt werden, dass die Variante CYP2C9*2 hinsichtlich der Hydroxylierungsaktivität der meisten Medikamente im Gegensatz zu CYP2C9*3 weniger vermindert ist (Kirchheiner et al. 2004). Andererseits zeigten die Ergebnisse zweier früherer in-vitro-Studien zu THC jedoch annähernd gleiche Effekte der Varianten CYP2C9*2 und CYP2C9*3 im Hinblick auf die Umwandlung von THC zu OH-THC. Deutlich wurde dies anhand der intrinsischen Clearance, die für CYP2C9*2 $0.9 \mu \mathrm{L} / \mathrm{min} / \mathrm{pmol}$ und für CYP2C9*3 $0.8 \mu \mathrm{L} / \mathrm{min} / \mathrm{pmol}$ betrug (Bland et al. 2005, Watanabe et al. 2007). Dies steht im Widerspruch zu den Daten der vorliegenden Studie. Jedoch sind jene Enzyme, die in weitere Reaktionsschritte von THC zu OH-THC einbezogen sind, in menschlichen Lebermikrosomen erst wenig untersucht worden. Auch wenn der funktionale Effekt der CYP2C9*2-Variante durch Veränderungen in der Interaktion mit der Cytochrom-P450-Reduktase zu entstehen scheint (Crespi und Miller 1997), könnten Unterschiede in der Reduktaseaktivität, bedingt durch stöchiometrische Unterschiede bei rekombinanten und humanen Mikrosomen, als mögliche Erklärung für die Diskrepanzen verantwortlich sein. Zur Veranschaulichung sind die Daten von Bland et al. (2005) denen unserer Studie in Tabelle 18 gegenübergestellt. 
Tab. 18 Einfluss des CYP2C9-Genpolymorphismus auf die Clearance von THC

\begin{tabular}{cccccc}
\hline & Bland et al. 2005 & \multicolumn{3}{c}{ Daten der vorliegenden Studie } \\
\hline Genotyp & $\left.\mathrm{Cl}_{\text {int }}[\mathrm{\mu L} / \mathbf{m i n} / \mathbf{p m o l})\right]$ & $\%$ & Genotyp & Cl/F [L/h] & $\%$ \\
\hline CYP2C9*1 & 3.0 & 100 & CYP2C ${ }^{*} 1 /{ }^{*} 1$ & 932 & 100 \\
CYP2C9*2 & 0.9 & 30 & CYP2C ${ }^{*} 2 /{ }^{*} 2$ & 928 & 100 \\
CYP2C9*3 & 0.8 & 27 & CYP2C9*3/*3 & 365 & 39 \\
\hline
\end{tabular}

$\mathrm{Cl}_{\text {int, }}$ intrinsische Clearance von THC in Lebermikrosomen (in vitro); $\mathrm{Cl} / \mathrm{F}$, Clearance dividiert durch Bioverfügbarkeit

\subsubsection{Einfluss des CYP2C9-Genpolymorphismus auf die Urinkonzentrationen von $\mathrm{COOH}-\mathrm{THC}$}

Bezüglich der Ausscheidung von $\mathrm{COOH}-\mathrm{THC}$ in den Urin ergaben sich Unterschiede in Abhängigkeit des CYP2C9-Genotyps. Einerseits war die Ausscheidung umso größer, je mehr CYP2C9*2-Allele die Probanden trugen (Abbildung 22), andererseits war sie umso kleiner, je mehr CYP2C9*3-Allele sie trugen (Abbildung 24). Beide Unterschiede waren statistisch signifikant. Die verminderte Ausscheidung in Abhängigkeit der Zahl an CYP2C9*3-Allelen lässt sich damit erklären, dass bei Trägern des Genotyps CYP2C9*3/*3 im Vergleich zu Trägern des Genotyps CYP2C9*1/*1 auch signifikant weniger THC zu COOH-THC metabolisiert wurde $\left(C_{\max } 4.8 \mu \mathrm{g} / \mathrm{L}\right.$ bei ${ }^{*} 1 /{ }^{*} 1,25.7 \mu \mathrm{g} / \mathrm{L}$ bei $\left.{ }^{*} 3 /{ }^{*} 3\right)$. Demzufolge wurde auch weniger ausgeschieden. Die Analysen unserer Urindaten zum CYP2C9*2-Allel deuteten sehr stark darauf hin, dass Träger dieser Variante THC beschleunigt metabolisieren. Allerdings ist hier zu berücksichtigen, dass die CYP2C9*2-Variante sehr eng mit einer Genvariante des benachbart lokalisierten CYP2C8-Gens gemeinsam vererbt wird (Vormfelde et al. 2007). Dort ist speziell die Variante CYP2C8*3 eng mit CYP2C9 gekoppelt, so dass die Urindaten auch so interpretiert werden können, dass das Enzym CYP2C8 an dieser Stelle den Metabolismus katalysiert. Um dies endgültig zu klären, müssten weitere biochemische in-vitro-Untersuchungen mit den CYP2C9- und CYP2C8-Varianten durchgeführt werden. 


\subsection{Bedeutung der Konzentrationsdaten für die forensische Begutachtung}

Die Ergebnisse der vorliegenden Studie könnten in der forensischen Medizin von Bedeutung sein. Neben Alkohol ist THC in vielen Ländern eine häufige Ursache von Beeinträchtigungen im Straßenverkehr (Giroud et al. 2006; Khiabani et al. 2006). Es gibt Daten, nach denen Cannabis eine erhebliche Rolle hinsichtlich tödlicher Autounfälle spielt (Drummer et al. 2004). Bei Verkehrskontrollen sind erhöhte Blutspiegel von THC nach dem Konsum von Alkohol ein häufiger Grund für Bußgeldund Strafverfahren. Auch gibt es einzelne Unternehmen, die Mitarbeiter auf THC-Konsum anhand von Blut- oder Urinproben untersuchen, zumindest dann, wenn der Verdacht des Missbrauchs besteht.

Da bei langsamen Metabolisierern, insbesondere Trägern zweier CYP2C9*3-Allele, die Möglichkeit einer verlängerten Sedierung durch THC besteht (Kapitel 3.8.2), könnte im Falle des illegalen Cannabiskonsums das Wissen um den CYP2C9Genotyp und seinen Einfluss auf den Metabolismus von THC von Bedeutung sein. Sowohl $\mathrm{COOH}-\mathrm{THC}$, als auch das Verhältnis von $\mathrm{COOH}-\mathrm{THC}$ zu THC werden in der forensischen Medizin zur Bewertung von Cannabiskonsum sowie hinsichtlich des Aufnahmezeitpunktes von THC zurate gezogen (Huestis et al. 2006; Musshoff und Madea 2006). Bei Trägern des Genotyps CYP2C9*3/*3 können im Gegensatz zu Trägern der Variante CYP2C ${ }^{*} 1{ }^{*} 1$ infolge verminderter CYP2C9-katalysierter Bildung des Metaboliten $\mathrm{COOH}-\mathrm{THC}$, deutlich niedrigere $\mathrm{COOH}-\mathrm{THC}$ Konzentrationen gefunden werden. Betrachtet man den Quotienten aus $\mathrm{COOH}-\mathrm{THC}$ und THC, der wie bereits erwähnt in der forensischen Medizin hinsichtlich des Konsumverhaltens von Cannabis ebenfalls von Bedeutung ist, zeigen sich ähnliche Ergebnisse. Langsame Metabolisierer wiesen bei identischem Aufnahmezeitpunkt von THC erheblich niedrigere Quotienten auf als schnelle Metabolisierer (Abbildung 13). So stieg er 48 Stunden nach Medikamentengabe bei Trägern der Variante $C Y P 2 C 9 * 1 /{ }^{*} 1$ auf etwa 120 an, während er für den $C Y P 2 C 9 * 3 / * 3-G e n o t y p$ lediglich einen Wert von etwa 10 erreichte. Demzufolge kann die Verwendung des Quotienten aus $\mathrm{COOH}-\mathrm{THC}$ und THC in der forensischen Medizin zu falschen Erkenntnissen führen. Hier erscheint es sinnvoll, die Beachtung der genotypbasierten Pharmakokinetik von THC bei der Validierung forensischer Testverfahren in Betracht zu ziehen. 
Auch die Ergebnisse der Urinanalyse könnten für die forensische Medizin von Bedeutung sein. Der CYP2C9-Polymorphismus nimmt nicht nur auf die Plasmakonzentrationen von THC und seinen Metaboliten Einfluss, sondern, wie bereits dargestellt, auch auf das Vorhandensein von COOH-THC im Urin. Dies kann die Ergebnisse von Urinschnelltests, welche auch auf dem Vorhandensein von $\mathrm{COOH}-\mathrm{THC}$ basieren (Grotenhermen 2003), hinsichtlich des Aufnahmezeitpunktes von THC beeinflussen. So könnte bei Trägern zweier CYP2C9*3-Allele das $\mathrm{COOH}-\mathrm{THC}$ über einen kürzeren Zeitraum detektierbar sein, als bei Trägern des Genotyps CYP2C9*2/*2.

\subsection{Beziehungen zwischen Pharmakogenetik, Pharmakokinetik und Pharmakodynamik}

In diesem Abschnitt sollen zunächst die in der Studie beobachteten Wirkungen von Dronabinol analysiert werden. Des Weiteren soll diskutiert werden, in welcher Beziehung die Blutkonzentrationsverläufe (Pharmakokinetik) zu den Wirkungen von Dronabinol (Pharmakodynamik) stehen. Der zweifelsfrei bestehende Zusammenhang der verschiedenen Genvarianten (Pharmakogenetik) mit der Pharmakokinetik und mit den entsprechenden Wirkungsdaten ist in Abbildung 44 dargestellt. Unklar bleibt dabei aber, in welchem Umfang die Pharmakokinetik durch die genannten Genvarianten bestimmt wird und inwieweit die Pharmakokinetik Einfluss auf die Pharmakodynamik nimmt. 


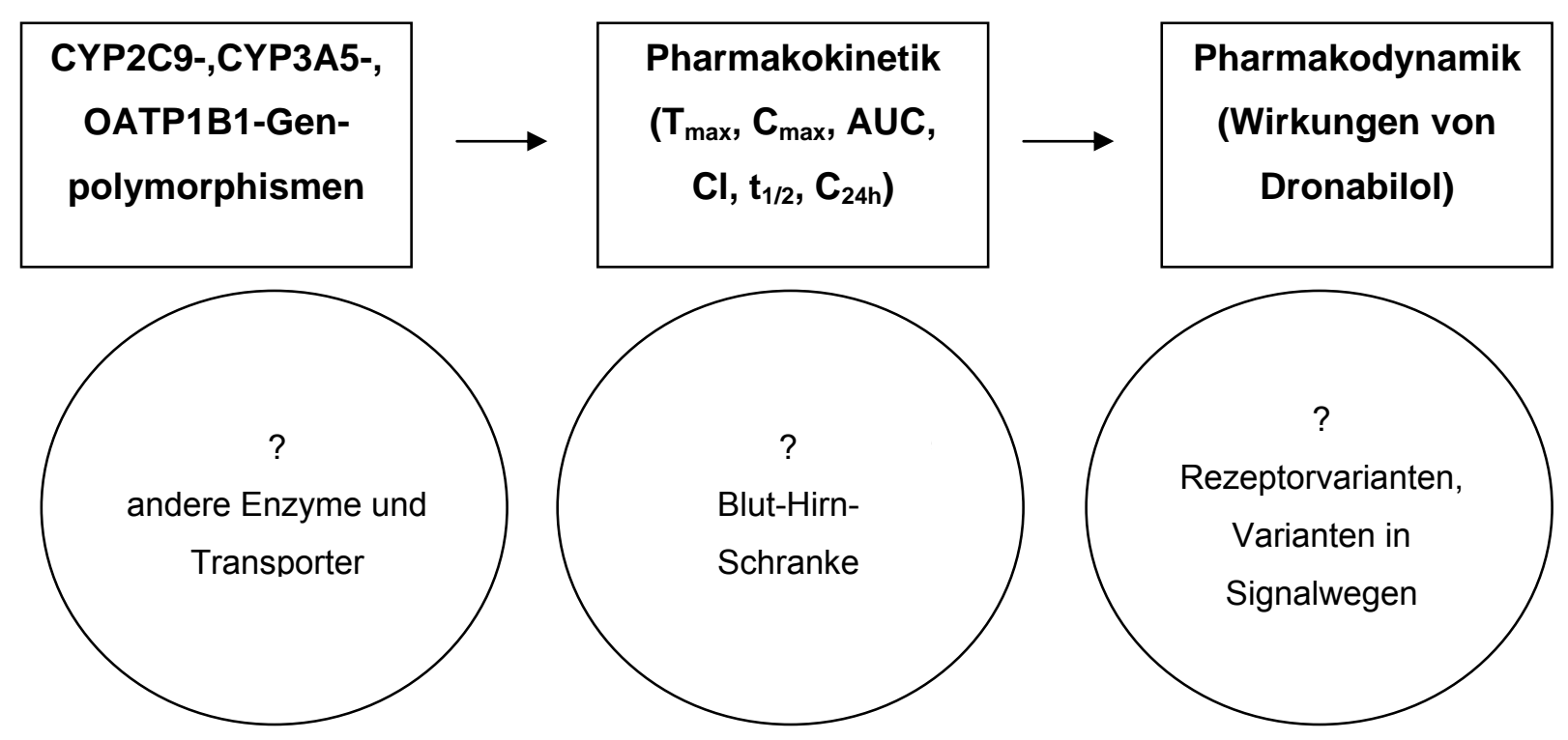

Abb. 44 Beziehung zwischen Pharmakogenetik, Pharmakokinetik und Pharmakodynamik; Der obere Abschnitt zeigt die im Rahmen der Studie analysierten Zusammenhänge, die Kreise weisen auf weitere mögliche Einflussfaktoren hin, die aus methodischen Gründen, beispielsweise weil sie noch nicht bekannt sind oder weil die Fallzahl mit $n=43$ nur begrenzt war, hier nicht analysiert wurden.

\subsubsection{Bedeutung des Endocannabinoidsystems}

Die Wirkungen von Dronabinol und anderen Cannabinoiden werden weitgehend über Cannabinoidrezeptoren (CNR-, CB-Rezeptoren) vermittelt. Zusammen mit ihren endogenen Liganden, den endogenen Cannabinoiden, zählen sie zum Endocannabinoidsystem.

Bis zum heutigen Zeitpunkt wurden bei Menschen zwei Cannabinoidrezeptoren detektiert: der CNR1- und der CNR2-Rezeptor. CNR2-Rezeptoren sind auf den Zellen des Immunsystems zu finden und sind im Nervensystem wahrscheinlich nur soweit exprimiert, wie sich dort eingewanderte Immunzellen befinden. Hier gibt es Forschungsansätze hinsichtlich antiinflammatorischer und antineoplastischer Effekte (Grotenhermen 2003). Im Jahre 1991 isolierte die Arbeitsgruppe Gérard et al. erstmals eine cDNA aus dem menschlichen Hirnstamm, die für den CNR1-Rezeptor kodiert. Der CNR1-Rezeptor ist ein G-Protein gekoppelter Rezeptor, bestehend aus 472 Aminosäuren (Gérard et al. 1991). 1992 konnte Anandamid, ein Ethanolaminderivat der Arachidonsäure, als erstes Substrat des CNR1-Rezeptors isoliert werden (Devane et al. 1992). CNR1-Rezeptoren sind vorwiegend im zentralen Nervensystem lokalisiert. Sie vermitteln verschiedene Wirkungen. 
Gegenstand der Forschung sind die Bedeutungen für Lern- und Bewegungsprozesse, Neuroprotektion, Temperatursteuerung und Schmerzprozesse. So bewirken beispielsweise bei Stress ausgeschüttete endogene Cannabinoide eine opiatunabhängige Analgesie (Hohmann et al. 2005). Für den CNR1-Rezeptor sind genetische Polymorphismen (SNPs) beschrieben, die im Zusammenhang mit Fettleibigkeit bei Männern stehen (Russo et al. 2007). Dies legt nahe, dass sie bei der Steuerung von Appetit und Motilität von Bedeutung sind. Des Weiteren können SNPs mit unterschiedlicher Anfälligkeit für Suchtsymptome nach Cannabiskonsum, Alkoholabhängigkeit oder bestimmten Formen der Schizophrenie einhergehen (Schmidt et al. 2002; Ujike et al. 2002; Hopfer et al. 2006; Zuo et al. 2007). Genetische Variationen des CNR1-Rezeptors erhöhen das Risiko für Adipositas und modulieren den BMI in der europäischen Population (Benzinou et al. 2008). Die Arbeitsgruppe Peeters et al. stellte einen Zusammenhang zwischen der Genvariante G1422A des CNR1-Rezeptors und Fettleibigkeit bei Männern her (Peeters et al. 2007) Der CNR1-Rezeptorblocker Rimonabant wurde zur Behandlung von Adipositas in Deutschland 2006 zugelassen, jedoch Ende 2008 aufgrund gehäufter psychiatrischer Nebenwirkungen wieder vom Markt genommen.

CNR1-Rezeptoren vermitteln die psychischen und vegetativen Wirkungen von Delta-9-THC. Auf eine Analyse der CNR1-Rezeptoren wurde in unserer Studie verzichtet, da diese Varianten nach vorliegender Literatur wohl nur eine geringe funktionelle Bedeutung haben. Zumindest wird diese Frage sehr kontrovers diskutiert und bei der begrenzten Fallzahl unserer Studie wären daher keine weiteren definitiven Klärungen zur Bedeutung der beschriebenen CNR1-Varianten zu erwarten gewesen.

\subsubsection{Neuropsychiatrische Wirkungen von Dronabinol}

In unserer Studie wurden die zentralnervösen Wirkungen und Nebenwirkungen von Dronabinol durch psychometrische Tests erfasst. Wirkungen konnten anhand der psychometrischen Parameter Müdigkeit und Tatendrang gezeigt werden. Der Sedierungsgrad wurde zum einen durch den POMS-Test und zum anderen anhand der Stanford-Sedierungs-Skala analysiert. Beide Testverfahren konnten eine Zunahme der Müdigkeit in Abhängigkeit der Medikamentengabe verzeichnen (Kapitel 3.8.2 und 3.9.5) Hier handelte es sich um eine bekannte 
Medikamentenwirkung (Grotenhermen 2003). Die Zeitpunkte der maximalen Müdigkeit lagen bei etwa 2 bis 4 Stunden und korrelierten damit mit denen der maximalen Plasmakonzentrationen der psychoaktiven Substanzen $\mathrm{THC}$ und $\mathrm{OH}-$ THC. Ähnliche Werte zeigten sich im Hinblick auf den abnehmenden Tatendrang. Dieser erreichte etwa 3 bis 4 Stunden nach Medikamentengabe sein Minimum (Kapitel 3.9.5). Die Zeitpunkte des Auftretens (nach etwa 30 - 90 Minuten) und die Dauer der Wirkungen (4 - 12 Stunden) lagen dabei in einem Bereich, der auch von Grotenhermen in seinem Review (2003) beschrieben wurde. Betrachtet man die Pharmakogenetik, ließ sich bei der Stanford-Sedierungs-Skala ein Trend feststellen. Je mehr CYP2C9*3-Allele die Probanden trugen, desto höher war ihr Score (Abbildungen 28 und 29). Es kam also bei Trägern der Variante CYP2C9*3/*3 tendenziell zu einer stärken Sedierung, als bei Trägern der Variante CYP2C9*1/*1. $\mathrm{Da}$ die Zahl an CYP2C9*3-Allelen ein signifikanter Einflussfaktor der pharmakokinetischen Parameter $\mathrm{C}_{\max }, \mathrm{AUC}$ und $\mathrm{Cl} / \mathrm{F}$ von $\mathrm{THC}$ war und die maximalen THC-Konzentrationen bei den Probanden der homozygoten CYP2C9*3-Variante gegenüber denen der homozygoten CYP2C9*1-Variante im Mittel etwa doppelt so groß waren, ließ sich damit ein Zusammenhang nicht nur zwischen Pharmakogenetik und der Pharmakokinetik, sondern auch zur Pharmakodynamik zeigen. Bei der Messung der Müdigkeit mit Hilfe des POMSTestverfahrens zeigten sich hinsichtlich der verschiedenen Genotypen ähnliche Tendenzen. Bei der Untersuchung des Tatendrangs gab es hingegen keine Genotypbasierten Unterschiede.

Die weiteren psychometrischen Testverfahren zur Reaktionszeit (RT), zur Aufmerksamkeit (FLIM), zur Zeit- und Bewegungsantizipation (ZBA) und zum Kurzzeitgedächtnis (FVW) ergaben keine Unterschiede vom Baseline- zum Verumtag. Bei manchen Tests erfolgte gar eine Verbesserung der Testergebnisse, anstelle eines erwarteten Abfalls der kognitiven und motorischen Leistungen eintrat. Dies ist in erster Linie auf Lerneffekte während des Studienverlaufs zurückzuführen, obgleich durch vorheriges Einüben der Tests versucht wurde, diese Lerneffekte zu minimieren.

Unsere Studie konnte keinen Beweis dafür erbringen, dass die getesteten neuropsychiatrischen und vegetativen Wirkungen von Dronabinol durch den Genpolymorphismus des CYP2C9-Enzyms beeinflusst wurden. Lediglich für den Sedierungsscore ergaben sich entsprechende Tendenzen. Die fehlende Korrelation 
der anderen getesteten Parameter mit den Genpolymorphismen der untersuchten CYP-Enzyme und OATP-Transportproteine könnte unter anderem dadurch erklärt werden, dass die Probandenkohorte nicht groß genug war und keine Verblindung vorgenommen wurde. Auch gibt es möglicherweise, wie bereits erwähnt, eine Reihe weiterer Einflussfaktoren, die hier eine Rolle spielen können (Abbildung 44). Ein weiterer Grund für das Fehlen deutlicher psychischer Effekte lag mit hoher Wahrscheinlichkeit darin, dass die Dosierung der Testsubstanz Dronabinol aus Sicherheitsgründen relativ moderat angesetzt war. Mit der einmaligen Verabreichung von $15 \mathrm{mg}$ Dronabinol erreichten wir mediane THC-Plasmakonzentrationen von $2.7 \mu \mathrm{g} / \mathrm{L}\left(C Y P 2 C 9 * 1 /{ }^{*} 1\right)$ bis $6.3 \mu \mathrm{g} / \mathrm{L}(C Y P 2 C 9 * 3 / * 3)$. Welche Schwellendosen und minimalen Plasmaspiegel von THC zum Erreichen von psychischen Wirkungen nötig sind, wird in der Literatur kontrovers betrachtet. Cone und Huestis (1993) kamen zu dem Ergebnis, dass psychotrope Effekte von Delta-9-THC ab einer oralen Dosierung von $25 \mathrm{mg}$ auftreten. Die Probanden dieser Studie erreichten THC-Blutplasmaspiegel von 7-29 $\mu \mathrm{g} / \mathrm{L}$. Diese führten zu einem subjektiven „High“-Effekt, welcher sich in der Größenordnung von $50 \%$ des erreichbaren Maximums befand (Cone und Huestis 1993). Die Arbeitsgruppe Ramaekers et al. (2006) beschrieb deutliche Beeinträchtigungen im Straßenverkehr bereits bei THC-Plasmakonzentrationen im Bereich von $2-5 \mu \mathrm{g} / \mathrm{L}$. Die Aufgabe hier war, in einer Verkehrssimulation als KFZ-Führer die Spur zu halten. In dieser Studie wurden auch kognitive Leistungen getestet, es konnten jedoch keine wesentlichen Veränderungen beobachtet werden. Eine lineare Beziehung zwischen dem Ausmaß der Beeinträchtigung und der THC-Plasmakonzentration ließ sich nur im Ansatz nachweisen. Eine weitere Studie ergab eine Beeinträchtigung des Kurzzeitgedächtnisses und des Lernvermögens bei oraler Applikation von $15 \mathrm{mg}$ THC und mittleren maximalen Plasmakonzentrationen von $4.5 \mu \mathrm{g} / \mathrm{L}$. Hier blieben Wahrnehmung und Arbeitsgedächtnis unbeeinflusst (Curran et al. 2002).

Ein Grund dieser Vielzahl an unterschiedlichen Untersuchungsergebnissen ist sicher auch, dass in jeder Studie verschiedene Tests angewandt wurden, deren Resultate häufig nicht miteinander vergleichbar sind. 


\subsubsection{Vegetative Wirkungen von Dronabinol}

Bezüglich der vegetativen Wirkungen wurden in dieser Studie Veränderungen des Blutdrucks und der Herzfrequenz analysiert. Die Herzfrequenzen stiegen unter dem Einfluss von Dronabinol im Mittel um 10/min an. Die mittleren systolischen und diastolischen Blutdrücke fielen leicht ab. Dies entsprach den beschriebenen Wirkungen von THC (Grotenhermen 2003). Hinsichtlich dieser Effekte waren keine genotypbasierten Unterschiede zu beobachten. Auch eine Korrelation mit den pharmakokinetischen Parametern des THC war hier nicht möglich.

\subsubsection{Einfluss von Dronabinol auf das Riechvermögen}

In der Riechschleimhaut des Menschen kommen Cytochrom-P450-Enzyme vor (Ling et al. 2004). Auch die Subfamilie der CYP2C-Enzyme ist dort vertreten. Sie können am Metabolismus von Xenobiotika, also auch von Medikamenten, beteiligt sein und beispielsweise die Bioverfügbarkeit beeinflussen (Ding und Kaminsky 2003). Die Wahrnehmung von Gerüchen wird durch viele Neuromodulatoren beeinflusst. Es gibt Anlass zu der Annahme, dass die Modulation bereits im Riechepithel erfolgt. Das cannabinerge System ist an der Verarbeitung von Gerüchen beteiligt. Liganden der CNR1-Rezeptoren modulieren Neuronen der Riechrezeptoren (Czesnik et al. 2007).

Aufgrund dieser Erkenntnisse führten wir in unserer Studie einen Riechtest durch. Ziel war es, einen möglichen Einfluss der Studienmedikation Dronabinol auf die Riechschwelle der Probanden bei verschiedenen Geruchsstoffen explorativ zu untersuchen. Die Riechschwelle des Geruchsstoffes Ananas unterschied sich als einzige der fünf Düfte signifikant vom Baseline- zum Verumtag (Abbildung 42). Bezüglich der Duftstoffe Ananas, Vanille und Rose ergaben sich Unterschiede zwischen den Genotypen, aber keine einheitliche Korrelation zwischen genetisch bedingter CYP2C9-Aktivität und der Riechschwelle. Eine Erklärung für die Abhängigkeit der Riechschwelle vom CYP2C9-Genotyp könnte sein, dass Geruchsstoffe im Nasenepithel auch von CYP2C9 metabolisiert werden und unterschiedlich schneller metabolischer Abbau mit unterschiedlicher Geruchsempfindlichkeit einhergeht. Als eine weitere Erklärung wäre denkbar, dass CYP2C9 wahrscheinlich auch an der Regulation der Durchblutung der 
Nasenschleimhaut beteiligt ist (Kirchheiner und Brockmöller 2005) und hier ein Anschwellen das Geruchsvermögen beeinträchtigen kann.

Bedingt durch die Vielzahl der Geruchsstoffe war die Analyse lediglich exploratorischer Natur, so dass der Einfluss des CYP2C9-Genotyps auf das Riechvermögen gegebenenfalls in weiteren Studien bestätigt werden sollte.

\subsection{Ausblick und Verbesserungsmöglichkeiten bei zukünftigen Studien}

Zukünftige pharmakogenetische Studien mit THC sollten Analysen zum Einfluss von Membrantransportern, Rezeptorvarianten und Modifikationen in den sich anschließenden Signalkaskaden beinhalten. Neuere Daten geben Anlass zu der Annahme, dass der MDR1-Transporter am Effluxtransport von THC beteiligt ist (Bonhomme-Faivre et al. 2008), jedoch war der Effekt eher gering. In der vorliegenden Studie wurden OATP1B1-Transportproteine untersucht, die möglicherweise am Influxtransport von THC beteiligt sind. Es kam in Abhängigkeit der zwei untersuchten funktionellen OATP1B1-Genotypen jedoch nicht zu pharmakokinetischen Unterschieden für THC und seine Metaboliten $\mathrm{OH}-\mathrm{THC}$ und $\mathrm{COOH}-\mathrm{THC}$. Umwelteinflüsse und genetische Polymorphismen der noch zu bestimmenden THC-Transporter könnten zukünftig einen Beitrag dazu leisten, weitere signifikante Anteile der bisher unerklärten Variationen in der Pharmakokinetik von THC zu identifizieren. Enzyme, welche die Glukuronidierung des THC katalysieren, könnten durch mehrkernige aromatische Kohlenwasserstoffe induzierbar sein. Jedoch ist es bis zum heutigen Zeitpunkt nicht gelungen, die daran beteiligten spezifischen Isoenzyme zu identifizieren. Letztendlich könnte das Allel CYP2C9*3 für die Wirkungen und Nebenwirkungen von THC und auch für Arzneimittelinteraktionen relevant sein.

Bezüglich der Urinausscheidung von $\mathrm{COOH}-\mathrm{THC}$ waren in unserer Studie sowohl das Allel CYP2C9*3, als auch das CYP2C9*2-Allel von Bedeutung. Zur Verifizierung dieser Ergebnisse, wären weitere Studien sinnvoll, welche die Urinausscheidung von $\mathrm{COOH}-\mathrm{THC}$ in Abhängigkeit der CYP2C9*2- und -*3-Allele über einen längeren Zeitraum untersuchen müssten, als es in dieser Studie der Fall war. Des Weiteren wären größere Fallzahlen der Genotypen CYP2C9*2/*2 und CYP $2 C 9 * 3 / * 3$ wünschenswert. 
Ein interessanter Ansatzpunkt für zukünftige Studien könnte in der weiterführenden Untersuchung pharmakodynamischer Aspekte, in Abhängigkeit des CYP2C9-Genpolymorphismus liegen. In unserer Studie ergaben sich geringfügige Anzeichen, dass diesbezüglich ein Zusammenhang besteht. Da insgesamt nur wenige Wirkungen und Nebenwirkungen des Dronabinols zu beobachten waren, müsste in Zukunft möglicherweise eine höhere Dosierung als die in unserer Studie gewählte oder auch ein multiple-dose-Verfahren angewendet werden.

Als ein grundsätzlicher Kritikpunkt unserer Untersuchung kann das Studiendesign genannt werden. Gerade in der Wirkung psychotroper Medikamente kommt dem Placebo-Effekt eine große Bedeutung zu. Allerdings sind Placebo-kontrollierte Studien schwierig zu gestalten, da wirksame THC-Dosen doch recht reproduzierbar zu einer Sedierung führen. Damit bleibt den Versuchspersonen nicht verborgen, ob sie ein Verum oder ein Placebo einnehmen. Lösungen in dieser Situation wären Vergleiche mit anderen sedierenden Pharmaka. Allerdings bleibt dann das Ergebnis relativ zu den jeweiligen anderen Pharmaka.

Ein weiterer Aspekt ergibt sich bezüglich der Interaktion von THC mit anderen Arzneimitteln. Bland et al. (2005) konnten zeigen, dass es in vitro zu einer Interaktion von THC mit dem Antikonvulsivum Phenytoin kommen kann, da beide Arzneimittel über CYP2C-Enzyme metabolisiert werden. Das Resultat dieser Interaktion können erniedrigte Plasmaspiegel von Phenytoin sein. Bei Trägern des Genotyps CYP2C9*3/*3 wären entsprechend höheren Plasmakonzentrationen von THC gegenüber CYP2C9*1/*1-Allelträgern Phenytoin-Plasmakonzentrationen möglich, die noch weiter unter den erwarteten liegen können. Dieser Fakt könnte zu einem unerwarteten Therapieversagen führen, da Phenytoin nur über eine geringe therapeutische Breite verfügt. Kenntnis über den CYP2C9-Genotyp der Patienten könnten hier von Nutzen sein, sollten die in-vitro-Ergebnisse auch in vivo gelten. 


\section{Zusammenfassung}

Cannabis wird heutzutage weltweit konsumiert. Allein in Europa nehmen täglich mehrere Millionen Menschen Cannabis, zumeist als illegale Droge, zu sich. Doch auch als Medikament kommt dem Hauptinhaltsstoff der Cannabispflanze, Delta-9-Tetrahydrocannabinol (Delta-9-THC), eine wachsende Bedeutung zu.

Das polymorphe Enzym Cytochrom-P450-2C9 trägt nach gegenwärtigen Daten zum Metabolismus von fast $20 \%$ aller Medikamente bei. Neben dem Wildtyp-Allel $\left(\right.$ CYP2C $\left.{ }^{*} 1\right)$ existieren zwei weitere Allele $\left({ }^{*} 2,{ }^{*}\right)$, deren Bedeutung in der europäischen Bevölkerung hinsichtlich einer erniedrigten enzymatischen Aktivität bereits für eine Vielzahl von Medikamenten beschrieben wurde. Gleichfalls wurden für das Enzym CYP3A5 und für die OATP-Influxtransportproteine genetische Polymorphismen detektiert, die den Stoffwechsel von Medikamenten beeinflussen können.

Diese Studie sollte untersuchen, ob es durch genetische Variationen in den Enzymen CYP2C9 und CYP3A5 sowie in den OATP-Transportproteinen zu Unterschieden in der Pharmakokinetik und Pharmakodynamik von Dronabinol (Delta-9-THC) kommt.

Grundlage dieser Untersuchung war eine offene, humanpharmakologische Probandenstudie im Parallelgruppendesign, in der 43 gesunden Männern und Frauen einmalig $15 \mathrm{mg}$ Dronabinol oral appliziert wurde. Die Gruppen wurden aus einer größeren Stichprobe nach dem CYP2C9- und CYP3A5-Genotyp selektiert.

Die Ergebnisse unserer Studie konnten zeigen, dass es in Abhängigkeit des Allels CYP2C9*3 zu statistisch signifikanten Unterschieden in der Pharmakokinetik von THC und $\mathrm{COOH}-\mathrm{THC}$ kommt. Träger der Genotypen CYP2C $9{ }^{*} 1 /{ }^{*} 1,{ }^{*} 1 /{ }^{*} 3$ und ${ }^{*} 3 /{ }^{*} 3$ hatten mediane orale THC-Clearance-Raten von 932, 473 und 365 L/h. Des Weiteren ergaben sich geschlechtsspezifische Differenzen. Die mediane orale Clearance von THC war bei den Männern mit 899 L/h signifikant höher als bei den Frauen mit $604 \mathrm{~L} / \mathrm{h}$. Der Vergleich zwischen Trägern der Allele CYP2C9*2 und CYP2C9*1 sowie zwischen Trägern der Allele CYP3A5*3 und CYP3A5*1 ergab keine signifikanten pharmakokinetischen Unterschiede in den Plasmakonzentrationen. Dies galt ebenso für die untersuchten genetischen Varianten der OATP-Transportproteine. Bei der Quantifizierung des $\mathrm{COOH}-\mathrm{THC}$ im Urin erwiesen sich das CYP2C9*2- und auch das -*3-Allel als signifikante Determinanten. Je mehr CYP2C9*3-Allele die Probanden trugen, desto geringer war die kumulative 
Ausscheidung von $\mathrm{COOH}-\mathrm{THC}$ über 24 Stunden. Die Mediane betrugen bei Trägern von 0, 1 oder 2 CYP2C9*3-Allelen 128.2, 66.8 und $30.9 \mu \mathrm{g} / 24 \mathrm{~h}$. Bezüglich des CYP2C9*2-Allels zeigten sich entgegengesetzte Ergebnisse. Mit steigender Zahl an CYP2C9*2-Allelen stieg auch die 24-Stunden-Ausscheidung des $\mathrm{COOH}-\mathrm{THC}$ an. Bei Trägern von 0,1 oder 2 CYP2C9*2-Allelen ergaben sich mediane kumulative Ausscheidungen von $88.9,135.7$ und $177.3 \mu \mathrm{g} / 24 \mathrm{~h}$.

Mit Hilfe computergestützter Testverfahren sollten die psychiatrischen und kognitiven Wirkungen von Dronabinol erfasst werden. Es konnten insgesamt allenfalls mäßige pharmakodynamische Effekte beobachtet werden, welche unabhängig von den untersuchten genetischen Polymorphismen auftraten. Effekte des THC konnten durch die Stanford-Sedierungs-Skala und den POMS-Test nachgewiesen werden. Hinsichtlich der Stanford-Sedierungs-Skala waren die Effekte bei Trägern des CYP2C $9 * 3 / * 3-G e n o t y p s$ besonders ausgeprägt. Interessant war schließlich, dass das THC und der CYP2C9-Genoyp in einem in die Studie integrierten Pilotexperiment Einflüsse auf den Geruchssinn zeigten. Ein nach Ananas riechender Geruchsstoff wurde schlechter unter THC wahrgenommen und es fanden sich Unterscheide in der Wahrnehmungsschwelle zwischen den CYP2C9-Genotypen.

Die durch Polymorphismen des Enzyms CYP2C9 bedingten Unterschiede in der Pharmakokinetik von THC könnten in der forensischen Medizin im Hinblick auf Drogentests in Blut und Urin von Bedeutung sein. Auch können sich neue Erkenntnisse zu Cannabis im Straßenverkehr ergeben. In den genannten Sparten sowie auch im medizinischen Sektor kann es für langsame Metabolisierer, insbesondere Träger der Variante CYP2C9*3/*3, von Nutzen sein, ihren Genotyp zu kennen. Weitere Studien auch zur Identifizierung von eventuell relevanten Genpolymorphismen in Signalproteinen müssten mit noch größeren Fallzahlen und einer höheren THC-Dosis durchgeführt werden. 


\section{$6 \quad$ Anhang}

\subsection{DASS-G-Fragebogen}

Studienakronym: THC / Protokoll Nr.: 2

Studiennummer:|_I_I_| Initialen: FN: I_I MN: I_I LN: I_I

Studientag/TP._Screenina_Datum: dd:|_I_|mm:|_|_lyy:|_|_|

Verfasser des Fragebogens: Dr. Peter F. Lovibond,Untwersity of New South Wales, Australla;

Vertasser des Fragebogens: Dr. Peter F. Lovlbond, Untwersity or New
Deutsche Bearbeltung: Dr. Cecilla A. Essau, Universitat Manster

DASS-G Fragebogen

Bitte lesen Sie jede Aussage und kreisen Sie die Zahl 0, 1, 2 oder 3 ein, die angeben soll, wie sehr die Aussage während der letzten Woche auf Sie zutraf. Es gibt keine richtigen oder falschen Antworten. Versuchen Sie, sich spontan für eine Antwort zu entscheiden.

Die Rating-Skala ist wie folgt:

0 Traf gar nicht auf mich zu

1 Traf manchmal auf mich zu, oder zu einem gewissen Grad

2 Traf in beträchtlichem Maße auf mich zu, oder ziemlich oft

3 Traf sehr stark auf mich zu, oder die meiste Zeit

1 Ich bemerkte, dass ich mich über Kleinigkeiten aufregte

2 Ich spürte, dass mein Mund trocken war

3 Ich konnte ūberhaupt keine positiven Gefühle erleben

4 Ich hatte Atemprobleme (z.B. übermäßig schnelles Atmen, Atemlosigkeit ohne körperliche Anstrengung)

5 Ich konnte einfach nicht in Gang kommen

6 Ich tendierte dazu, auf Situationen überzureagieren

7 Ich fühlte mich zittrig (z.B. schwach in den Beinen)

8 Ich fand es schwierig, mich zu entspannen

9 Ich fand mich in Situationen wieder, die mich so āngstlich machten, dass ich sehr froh war, wenn sie vorbei waren

10 Ich hatte das Gefühl, nichts zu haben, auf das ich mich freuen konnte

11 Ich bemerkte, dass ich mich ziemlich schnell aufregte

12 Ich fühlte, dass ich eine Menge Nervenkraft verbrauchte

13 Ich fūhlte mich traurig und niedergedrückt

14 Ich bemerkte, dass ich ungeduldig wurde, wenn ich irgendwie aufgehalten wurde (z.B. im Fahrstuhl, an Ampeln, wenn man mich warten ließ)

15 Ich hatte das Gefühl, ohnmächtig zu werden

16 Ich fühlte, dass ich das Interesse an allem verloren hatte

17 Ich fühlte mich als Person nicht viel wert

18 Ich fand mich ziemlich empfindlich

\begin{tabular}{llll}
0 & 1 & 2 & 3 \\
0 & 1 & 2 & 3 \\
0 & 1 & 2 & 3 \\
0 & 1 & 2 & 3 \\
0 & 1 & 2 & 3 \\
0 & 1 & 2 & 3 \\
0 & 1 & 2 & 3 \\
0 & 1 & 2 & 3 \\
0 & 1 & 2 & 3 \\
0 & 1 & 2 & 3 \\
0 & 1 & 2 & 3 \\
0 & 1 & 2 & 3 \\
0 & 1 & 2 & 3 \\
0 & 1 & 2 & 3 \\
0 & 1 & 2 & 3 \\
0 & 1 & 2 & 3 \\
0 & 1 & 2 & 3 \\
0 & 1 & 2 & 3 \\
\hline 0 & 009 &
\end{tabular}

Contact ds@sehrt.de; cs@klinischepharmakologie.de

08.05 .2009

Clinical Pharmacology - Medical significance and influence of genetic polymorphisms of the enzyme cytochromeP450-2C9 and $3 \mathrm{~A} 5$ in plasma dronabinol concentration-time curves and effects of dronabinol

$$
\text { Seite } 1 \text { von } 2
$$


Studienakronym: THC / Protokoll Nr.: 2

Studiennummer:|_I_I_| Initialen: FN: I_I MN: I_I LN: I_|

Studientag/TP.:-Screenina

Datum: dd:|_|_|mm:|_|_|yy:|_|_|

\begin{tabular}{|c|c|c|c|c|c|}
\hline 19 & $\begin{array}{l}\text { Ich schwitzte spürbar (z.B. feuchte Hände), ohne dass hohe Temperaturen } \\
\text { herrschten oder dass ich mich körperlich anstrengte }\end{array}$ & 0 & 1 & 2 & 3 \\
\hline 20 & Ich fühlte mich grundlos ängstlich & 0 & 1 & 2 & 3 \\
\hline 21 & Ich hatte das Gefühl, dass das Leben sich nicht lohnt & 0 & 1 & 2 & 3 \\
\hline 22 & Ich fand es schwer, mich zu beruhigen & 0 & 1 & 2 & 3 \\
\hline 23 & Ich hatte Schluckbeschwerden & 0 & 1 & 2 & 3 \\
\hline 24 & Es schien, als könnte ich die Dinge, die ich tat, ūberhaupt nicht genießen & 0 & 1 & 2 & 3 \\
\hline 25 & $\begin{array}{l}\text { Ich war mir ūber meinen Herzschlag bewusst, ohne dass ich mich körperlich } \\
\text { angestrengt hatte (z.B. das Gefühl beschleunigten Herzschlags, das Gefühl, } \\
\text { dass der Herzschlag aussetzt) }\end{array}$ & 0 & 1 & 2 & 3 \\
\hline 26 & Ich fühlte mich niedergeschlagen und traurig & 0 & 1 & 2 & 3 \\
\hline 27 & Ich stellte fest, dass ich sehr reizbar war & 0 & 1 & 2 & 3 \\
\hline 28 & Ich fühlte mich einer Panik nahe & 0 & 1 & 2 & 3 \\
\hline 29 & Ich fand es schwer, mich zu beruhigen, wenn mich etwas geärgert hatte & 0 & 1 & 2 & 3 \\
\hline 30 & $\begin{array}{l}\text { Ich befürchtete, dass mich eine einfache, aber ungewohnte Aufgabe aus der } \\
\text { Bahn werfen würde }\end{array}$ & 0 & 1 & 2 & 3 \\
\hline 31 & Ich war nicht in der Lage, mich für irgendetwas zu begeistern & 0 & 1 & 2 & 3 \\
\hline 32 & $\begin{array}{l}\text { Ich fand es schwierig zu tolerieren, wenn ich bei einer Tätigkeit unterbrochen } \\
\text { wurde }\end{array}$ & 0 & 1 & 2 & 3 \\
\hline 33 & Ich war in einem Zustand nervöser Anspannung & 0 & 1 & 2 & 3 \\
\hline 34 & Ich fühlte mich ziemlich wertlos & 0 & 1 & 2 & 3 \\
\hline 35 & $\begin{array}{l}\text { Ich konnte nichts ertragen, das mich davon abhielt, in meiner Tätigkeit } \\
\text { fortzufahren }\end{array}$ & 0 & 1 & 2 & 3 \\
\hline 36 & Ich fühlte mich erschrocken & 0 & 1 & 2 & 3 \\
\hline 37 & Ich konnte nicht hoffnungsvoll in die Zukunft blicken & 0 & 1 & 2 & 3 \\
\hline 38 & Ich empfand das Leben als sinnlos & 0 & 1 & 2 & 3 \\
\hline 39 & Ich bemerkte, dass ich unruhig wurde & 0 & 1 & 2 & 3 \\
\hline 40 & $\begin{array}{l}\text { Ich machte mir Sorgen ūber Situationen, in denen ich in Panik geraten und } \\
\text { mich zum Trottel machen könnte }\end{array}$ & 0 & 1 & 2 & 3 \\
\hline 41 & Ich zitterte (z.B. an den Händen) & 0 & 1 & 2 & 3 \\
\hline 42 & Es fiel mir schwer, die Initiative aufzubringen, Dinge zu tun & 0 & 1 & 2 & 3 \\
\hline
\end{tabular}




\subsection{HADS-D-Fragebogen}

Studienakronym: THC / Protokoll Nr.: 2

Studiennummer:|_I_I_I Initialen: FN: I_I MN: I_I LN: I_I

Studientag/TP._Screenina_Datum: dd:I_I_|mm:|_l_lyy:|_|_|

\section{HADS-D Fragebogen}

Wir bitten Sie, jede Frage zu beantworten, und zwar so, wie es für Sie persönlich in der letzten Woche am ehesten zutraf. Machen Sie bitte nur ein Kreuz pro Frage, und lassen Sie bitte keine Frage aus! Uberlegen Sie bitte nicht lange, sondern wählen Sie die Antwort aus, die Ihnen auf Anhieb am zutreffendsten erscheint!

\begin{tabular}{|c|c|c|c|}
\hline 1 & $\begin{array}{l}\text { Ich fühle mich angespannt oder } \\
\text { ūberreizt } \\
\square \text { meistens } \\
\square \text { oft } \\
\square \text { von Zeit zu Zeit / gelegentlich } \\
\square \text { überhaupt nicht }\end{array}$ & 6 & $\begin{array}{l}\text { Ich fühle mich glücklich } \\
\square \text { überhaupt nicht } \\
\square \text { selten } \\
\square \text { manchmal } \\
\square \text { meistens }\end{array}$ \\
\hline 2 & $\begin{array}{l}\text { Ich kann mich heute noch so freuen } \\
\text { wie früher } \\
\square \text { ganz genau so } \\
\square \text { nicht ganz so sehr } \\
\square \text { nur noch ein wenig } \\
\square \text { kaum oder gar nicht }\end{array}$ & 7 & $\begin{array}{l}\text { Ich kann behaglich dasitzen und mich } \\
\text { entspannen } \\
\square \text { ja, natürlich } \\
\square \text { gewöhnlich schon } \\
\square \text { nicht oft } \\
\square \text { überhaupt nicht }\end{array}$ \\
\hline 3 & $\begin{array}{l}\text { Mich überkommt eine ängstliche } \\
\text { Vorahnung, dass etwas Schreckliches } \\
\text { passieren könnte } \\
\square \text { ja, sehr stark } \\
\square \text { ja, aber nicht allzu stark } \\
\square \text { etwas, aber es macht mir keine } \\
\text { Sorgen } \\
\square \text { überhaupt nicht }\end{array}$ & 8 & $\begin{array}{l}\text { Ich fühle mich in meinen Aktivitäten gebremst } \\
\square \text { fast immer } \\
\square \text { sehr oft } \\
\square \text { manchmal } \\
\square \text { überhaupt nicht }\end{array}$ \\
\hline 4 & $\begin{array}{l}\text { Ich kann lachen und die lustige Seite } \\
\text { der Dinge sehen } \\
\square \text { ja, so viel wie immer } \\
\square \text { nicht mehr ganz so viel } \\
\square \text { inzwischen viel weniger } \\
\square \text { überhaupt nicht }\end{array}$ & 9 & $\begin{array}{l}\text { Ich habe manchmal ein ängstliches Gefühl in der } \\
\text { Magengegend } \\
\square \text { überhaupt nicht } \\
\square \text { gelegentlich } \\
\square \text { ziemlich oft } \\
\square \text { sehr oft }\end{array}$ \\
\hline 5 & $\begin{array}{l}\text { Mir gehen beunruhigende Gedanken } \\
\text { durch den Kopf } \\
\square \text { einen Großteil der Zeit } \\
\square \text { verhältnismäßig oft } \\
\square \text { von Zeit zu Zeit, aber nicht allzu oft } \\
\square \text { nur gelegentlich/nie }\end{array}$ & 10 & $\begin{array}{l}\text { Ich habe das Interesse an meiner äußeren } \\
\text { Erscheinung verloren } \\
\square \text { ja, stimmt genau } \\
\square \text { ich kümmere mich nicht so sehr darum, wie ich } \\
\text { sollte } \\
\square \text { möglicherweise kümmere ich mich zu wenig } \\
\text { darum } \\
\square \text { ich kümmere mich so viel darum wie immer }\end{array}$ \\
\hline
\end{tabular}


Studienakronym: THC / Protokoll Nr.: 2

Studiennummer:|_I_I_| Initialen: FN: I_I MN: I_I LN: I_|

Studientag/TP.:-Screenina

Datum: dd:|_|_|mm:|_|_|yy:|_|_|

\begin{tabular}{|c|c|c|c|}
\hline$\overline{11}$ & $\begin{array}{l}\text { Ich fühle mich rastlos, muß immer in } \\
\text { Bewegung sein } \\
\square \text { ja, tatsächlich sehr } \\
\square \text { ziemlich } \\
\square \text { nicht sehr } \\
\square \text { überhaupt nicht }\end{array}$ & 13 & $\begin{array}{l}\text { Mich überkommt plötzlich ein panikartiger } \\
\text { Zustand } \\
\square \text { ja, tatsächlich sehr oft } \\
\square \text { ziemlich oft } \\
\square \text { nicht sehr oft } \\
\square \text { überhaupt nicht }\end{array}$ \\
\hline$\overline{12}$ & $\begin{array}{l}\text { Ich blicke mit Freude in die Zukunft } \\
\square \text { ja, sehr } \\
\square \text { eher weniger als früher } \\
\square \text { viel weniger als früher } \\
\square \text { kaum bis gar nicht }\end{array}$ & 14 & $\begin{array}{l}\text { Ich kann mich an einem guten Buch, einer } \\
\text { Radio- oder Fernsehsendung freuen } \\
\square \text { oft } \\
\square \text { manchmal } \\
\square \text { eher selten } \\
\square \text { sehr selten }\end{array}$ \\
\hline
\end{tabular}

Clinical Pharmacology - Medical significance and influence of genetic polymorphisms of the enzyme cytochromeP450-2C9 and 3A5 in plasma dronabinol concentration-time curves and effects of dronabinol

Seite 2 won 2 


\subsection{Fragebogen 1}

Studienakronym: THC / Protokoll Nr.: 2

Studiennummer:|_I_I_| Initialen: FN: |_| MN: I_I LN: I_|

Studientag/TP. Datum: dd:|_I_|mm:|_|_|yy:|_|_|

Bei der Bearbeitung des CRF ist die Dokumentationsreihenfolge einzuhalten. Vervollständigung von oben nach unten und von links nach rechts.

Folgende Angaben sind vor Beginn und am Ende jedes Studientages zu ergänzen (Fragebogen 1) - Untersucher

Ja Nein

1. Haben Sie gesundheitliche Beschwerden, oder fühlen Sie sich krank? $\square$

2. Haben Sie heute früh bereits Nahrung zu sich genommen? $\quad \square \quad \square$

Körpertemperatur: I_I_l,I_l ${ }^{\circ} \mathrm{C}$

Die Körpertemperaturmessung erfolgte: $\quad$ oral $\quad$ Axilla $\square$ Ohr $\square$

Bemerkungen:

Aus medizinischer Sicht bestehen keine Bedenken an der Studie teilzunehmen!

Name

Unterschrift 
Studienakronym: THC / Protokoll Nr.: 2

Studiennummer:|_I_I_| Initialen: FN: |_| MN: I_I LN: I_|

Studientag/TP.

Datum: dd:|_I_|mm:|_|_|yy:|_|_|

\section{Proband}

Bitte füllen Sie folgenden Fragebogen aus. Bitte beachten Sie, dass sich die Fragen auf die vergangenen 24 Stunden beziehen:

Bitte füllen Sie den Fragebogen in dieser Weise aus: $\mathbf{X}$, und ergänzen Sie evtl. weitere Besonderheiten. Falls eine Korrektur notwendig wird, so benutzen Sie bitte einen Unterstrich, um die korrekte Wahl anzuzeigen: 4 . Bitte nur ein Kreuz je Zeile setzen.

0: nein/gar nicht; 1: andeutungsweise; 2: leicht; 3: deutlich; 4: stark; 5: sehr stark

\begin{tabular}{|c|c|}
\hline $\begin{array}{l}\text { Wurde Ihnen beim Aufstehen } \\
\text { schwindelig? }\end{array}$ & 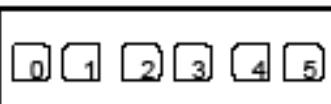 \\
\hline Ist Ubelkeit aufgetreten? & 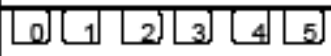 \\
\hline Mussten Sie sich erbrechen? & \begin{tabular}{|l|l|l|l|l|l}
0 & 1 & 2 & 2 & 3 & $(4)$ \\
\end{tabular} \\
\hline Hatten Sie Durchfall? & {$[0[1[2][3][4]$} \\
\hline Hatten Sie Verstopfung? & 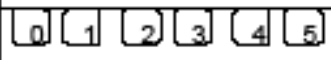 \\
\hline $\begin{array}{l}\text { Hatten Sie Schwierigkeiten beim } \\
\text { Wasserlassen? }\end{array}$ & 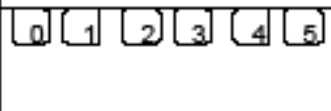 \\
\hline $\begin{array}{l}\text { Sind Veränderungen an der Haut } \\
\text { aufgetreten? }\end{array}$ & 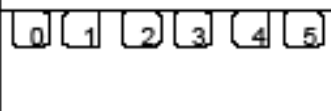 \\
\hline $\begin{array}{l}\text { Hat sich eine bestehende Allergie } \\
\text { verstärkt? }\end{array}$ & 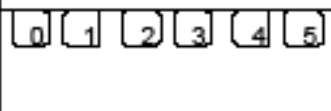 \\
\hline $\begin{array}{l}\text { War Ihr Hörvermögen eingeschränkt? } \\
\text { (zB Ohrensausen) }\end{array}$ & 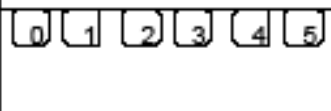 \\
\hline Hatten Sie Sehstörungen? & $6[4[2][3][4[5]$ \\
\hline $\begin{array}{l}\text { Waren Ihre Augen gereizt oder } \\
\text { trocken? } \\
\end{array}$ & 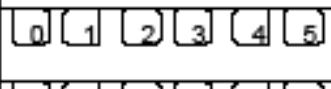 \\
\hline War Ihr Mund trocken? & 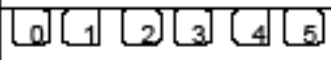 \\
\hline Hatten Sie eine verstopfte Nase? & 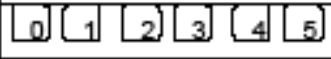 \\
\hline Sind Kopfschmerzen aufgetreten? & \begin{tabular}{|l|l|l|l|l}
0 & {$[1$} & $2]$ & 3 & 4 \\
\end{tabular} \\
\hline
\end{tabular}

Contact ds@sehrt.de; cs@klinischepharmakologie.de

08.05.2009

Clinical Pharmacology - Medical significance and influence of genetic polymorphisms of the enzyme cytochromeP450-2C9 and 3A5 in plasma dronabinol concentration-time curves and effects of dronabinol 
Studienakronym: THC / Protokoll Nr.: 2

Studiennummer:|_I_I_| Initialen: FN: |_| MN: |_I LN: I_|

Studientag/TP.

Datum: dd:|_|_|mm:|_|_|yy:|_|_|

\begin{tabular}{|c|c|}
\hline Konnten Sie ein- oder durchschlafen? & 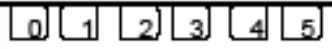 \\
\hline Hatten Sie Alpträume? & $0][1] 2][3] 4[5]$ \\
\hline Waren Sie nervös oder ängstlich? & {$[0[1][2][3] 4[5]$} \\
\hline Waren Sie vergesslich? & 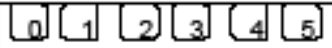 \\
\hline Waren Sie übel gelaunt? & 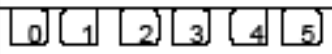 \\
\hline Hatten Sie ein Hochgefühl erlebt? & 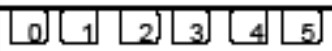 \\
\hline $\begin{array}{l}\text { Hatten Sie das Gefühl, Dinge } \\
\text { gesehen zu haben, die andere nicht } \\
\text { sehen konnten oder Geräusche zu } \\
\text { hören, die andere nicht hören } \\
\text { konnten? }\end{array}$ & $0] \square$ \\
\hline Hatten Sie kalte Hände / Füße? & {$[0][1][2][3][4] 5$} \\
\hline $\begin{array}{l}\text { Fiel es Ihnen schwer, sich zu } \\
\text { konzentrieren? }\end{array}$ & {$[0][1][2][3][4][5]$} \\
\hline
\end{tabular}

Bitte ergänzen Sie hier evtl. weitere Angaben:

Contact ds (6) sehrt.de; cs\&klinischepharmakologie.de

Clinical Pharmacology - Medical significance and influence of genetic polymorphisms of the enzyme cytochromeP450-2C9 and 3A5 in plasma dronabinol concentration-time curves and effects of dronabinol 


\subsection{Fragebogen 2}

Studienakronym: THC / Protokoll Nr.: 2

Studiennummer:|_I_I_| Initialen: FN: I_I MN: I_I LN: I_|

Studientag/TP. Datum: dd:|_|_|mm:|_|_|yy:|_|_|

Bitte ergänzen Sie den Fragebogen, der sich auf lhre aktuelle Situation bezieht:

\section{(Fragebogen 2)}

Bitte füllen Sie den Fragebogen in dieser Weise aus: $\mathbf{X}$, und ergänzen Sie evtl. weitere Besonderheiten. Falls eine Korrektur notwendig wird, so benutzen Sie bitte einen Unterstrich, um die korrekte Wahl anzuzeigen: 4 . Bitte nur ein Kreuz je Zeile setzen.

0: nein/gar nicht; 1: andeutungsweise; 2: leicht; 3: deutlich; 4: stark; 5: sehr stark

\begin{tabular}{|c|c|}
\hline $\begin{array}{l}\text { Fühlen Sie sich körperlich schwach, müde } \\
\text { und abgeschlagen? }\end{array}$ & $0 \square \square \square \square$ \\
\hline Sind Sie nervös? & $[0][1] 2][3][4] 5]$ \\
\hline Sind Sie ängstlich? & {$[a][1][2][3][4][5]$} \\
\hline Empfinden Sie ein „High"-Gefühl? & $[Q][1][2][3][4] 5]$ \\
\hline $\begin{array}{l}\text { Haben Sie das Gefühl, Dinge zu sehen, die } \\
\text { für andere nicht sichtbar sind? }\end{array}$ & 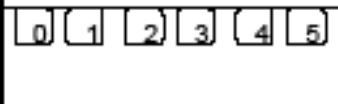 \\
\hline $\begin{array}{l}\text { Haben Sie das Gefühl, Stimmen zu hören, } \\
\text { die für andere nicht hörbar sind? }\end{array}$ & $60\left[\begin{array}{ll}0 \\
0\end{array}\right.$ \\
\hline Vergeht die Zeit subjektiv anders? & \begin{tabular}{|ll}
$J a \square \quad$ Nein $\square$ \\
\end{tabular} \\
\hline Falls ja, schneller? & {$[0][1][2][3][4][5]$} \\
\hline Falls ja, langsamer? & \begin{tabular}{|l|l|l|l|l|}
$0]$ & \\
\end{tabular} \\
\hline $\begin{array}{l}\text { Fällt es Ihnen schwer, sich zu } \\
\text { konzentrieren? }\end{array}$ & $\mid \theta[1[2][3][4] 5]$ \\
\hline Haben Sie kalte Hände/Füße? & $\mid \theta[1[2][3][4] 5]$ \\
\hline Ist Ihr Mund trocken? & $[Q][1][2][3][4] 5]$ \\
\hline Haben Sie Kopfschmerzen? & 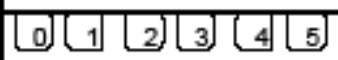 \\
\hline Ist Ihnen schwindelig? & $[0[1] 2][3][4] 5]$ \\
\hline Ist Ihnen übel? & {$[6][1][2][3][4][5]$} \\
\hline
\end{tabular}


Studienakronym: THC / Protokoll Nr.: 2

Studiennummer:|_I_I_| Initialen: FN: |_| MN: I_I LN: I_|

Studientag/TP.

Datum: dd:|_|_|mm:|_|_|yy:|_|_|

Bitte ergänzen Sie hier evtl. weitere Angaben:

Bitte nur in einer am besten zutreffenden Zeile rechts ankreuzen:

Sie fühlen sich sich sehr aktiv, vital, aufmerksam bzw. sehr wach.

Sie fühlen sich auf hohem Level, aber nicht maximal. Sie sind fähig, sich zu konzentrieren.

Sie fühlen sich wach, aber sind entspannt. Sie reagieren normal, aber erreichen nicht vollständige Aufmerksamkeit.

Sie fühlen sich etwas schläfrig. Es stellt sich beginnender Interessenverlust ein.

Sie sind schläfrig. Ihr Interesse, wach zu bleiben, wird geringer. Sie fühlen sich verlangsamt.

Sie sind schläfrig und fühlen sich "benebelt". Sie kämpfen gegen den Schlaf

an. Sie haben das Bedürfnis, sich hinlegen zu wollen. 
Studienakronym: THC/ Protokoll Nr.: 2

Studiennummer:|_I_I_| Initialen: FN: |_| MN: I_I LN: I_|

Studientag/TP.

Datum: dd:|_I_|mm:|_|_|yy:|_|_|

\section{Untersucher}

\section{Stanford-Sleepiness Scale}

Bitte zutreffendes in der rechten Spalte ankreuzen

\begin{tabular}{|l|l|l|}
\hline & Grad der Schläfrigkeit & \\
\hline $\mathbf{1}$ & sich sehr aktiv, vital, aufmerksam bzw. sehr wach fühlen & \\
\hline $\mathbf{2}$ & $\begin{array}{l}\text { sich auf hohem Level fühlen, aber nicht maximal; fähig sich zu } \\
\text { konzentrieren }\end{array}$ & $\begin{array}{l}\text { sich wach fühlen, aber entspannt sein; normal reagierend, aber } \\
\text { nicht vollständige Aufmerksamkeit erreichend }\end{array}$ \\
\hline $\mathbf{4}$ & Etwas schläfrig, beginnender Interessenverlust & \\
\hline $\mathbf{5}$ & Schläfrig; Interesse, wach zu bleiben, wird geringer; verlangsamt & \\
\hline $\mathbf{6}$ & $\begin{array}{l}\text { Schläfrig, „benebelt", gegen den Schlaf ankämpfend bzw. } \\
\text { Bedürfnis, sich hinlegen zu wollen }\end{array}$ & \\
\hline $\mathbf{7}$ & $\begin{array}{l}\text { Nicht länger gegen den Schlaf ankämpfend; baldiges Einsetzen } \\
\text { des Schlafes bzw. traumartige Gedanken }\end{array}$ & \\
\hline $\mathbf{X}$ & Schlaf & \\
\hline
\end{tabular}

\begin{tabular}{|c|c|}
\hline Sind die Konjunktiven gerötet? & \begin{tabular}{|l|l|l|l|l|l|}
0 & $G$ & 2 & 3 & 4 & 5 \\
\end{tabular} \\
\hline Liegt eine Hautrötung vor? & \begin{tabular}{|l|l|l|l|l|l|}
0 & 1 & 2 & 3 & 4 & 5 \\
\end{tabular} \\
\hline Sind die Hände/Füße kalt? & 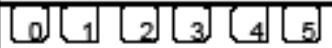 \\
\hline Neurologische Untersuchung (bitte Formblatt benutzen) & Ja $\square \quad$ Nein $\square$ \\
\hline
\end{tabular}

Ggf. Erläuterung o.g. Veränderungen bitte im zugehörigen CRF-Bogen des jeweiligen Messzeitpunktes angeben.

Untersucher:

Name Unterschrift 


\section{$7 \quad$ Literaturverzeichnis}

Aithal GP, Day CP, Kesteven PJ, Daly AK (1999): Association of polymorphisms in the cytochrome P450 CYP2C9 with warfarin dose requirement and risk of bleeding complications. Lancet $\underline{353}, 717-9$

Ben Amar M (2006): Cannabinoids in medicine: A review of their therapeutic potential. J Ethnopharmacol 105, 1-25

Benzinou M, Chèvre JC, Ward KJ, Lecoeur C, Dina C, Lobbens S, Durand $E$, Delplanque J, Horber FF, Heude B, Balkau B, Borch-Johnsen K, Jørgensen T, Hansen T, Pedersen O, Meyre D, Froguel P (2008): Endocannabinoid receptor 1 gene variations increase risk for obesity and modulate body mass index in European populations. Hum Mol Genet $\underline{17}, 1916-21$

Biecheler MB, Peytavin JF, Sam G, Facy F, Martineau H (2008): SAM survey on "drugs and fatal accidents": search of substances consumed and comparison between drivers involved under the influence of alcohol or cannabis. Traffic Inj Prev $\underline{9}, 11-21$

Bifulco M, Malfitano AM, Pisanti S, Laezza C (2008): Endocannabinoids in endocrine and related tumors. Endocr Relat Cancer 15, 391-408

Bland TM, Haining RL, Tracy TS, Callery PS (2005): CYP2C-catalyzed delta9tetrahydrocannabinol metabolism: kinetics, pharmacogenetics and interaction with phenytoin. Biochem Pharmacol 무, 1096-103

Bonhomme-Faivre L, Benyamina A, Reynaud M, Farinotti R, Abbara C (2008): Disposition of Delta tetrahydrocannabinol in CF1 mice deficient in mdr1a $\mathrm{P}$ glycoprotein. Addict Biol $\underline{13}$, 295-300

Bornheim LM, Lasker JM, Raucy JL (1992): Human hepatic microsomal metabolism of delta 1-tetrahydrocannabinol. Drug Metab Dispos 므, 241-6 
Brockmöller J, Kirchheiner J, Meisel C, Roots I (2000): Pharmacogenetic diagnostics of cytochrome P450 polymorphisms in clinical drug development and in drug treatment. Pharmacogenomics 1 , 125-51

Cone EJ, Huestis MA (1993): Relating blood concentrations of tetrahydrocannabinol and metabolites to pharmacologic effects and time of marijuana usage. Ther Drug Monit $\underline{15}, 527-32$

Crespi CL, Miller VP (1997): The R144C change in the CYP2C9*2 allele alters interaction of the cytochrome P450 with NADPH: cytochrome P450 oxidoreductase. Pharmacogenetics $\underline{7}, 203-10$

Croxford JL (2003): Therapeutic potential of cannabinoids in CNS disease. CNS Drugs $\underline{17}, 179-202$

Curran HV, Brignell C, Fletcher S, Middleton P, Henry J (2002): Cognitive and subjective dose-response effects of acute oral Delta 9-tetrahydrocannabinol (THC) in infrequent cannabis users. Psychopharmacology $\underline{164}, 61-70$

Czesnik D, Schild D, Kuduz J, Manzini I (2007): Cannabinoid action in the olfactory epithelium. Proc Natl Acad Sci U S A 104, 2967-72

Derendorf H, Gramatté T, Schäfer HG: Pharmakokinetik; Einführung in die Theorie und Relevanz für die Arzneimitteltherapie, 2. Auflage; Wissenschaftliche Verlagsgesellschaft mbH, Stuttgart 2002

Deutsch SI, Rosse RB, Connor JM, Burket JA, Murphy ME, Fox FJ (2008): Current status of cannabis treatment of multiple sclerosis with an illustrative case presentation of a patient with MS, complex vocal tics, paroxysmal dystonia, and marijuana dependence treated with dronabinol. CNS Spectr $\underline{13}, 393-403$

Devane WA, Hanus L, Breuer A, Pertwee RG, Stevenson LA, Griffin G, Gibson D, Mandelbaum A, Etinger A, Mechoulam R (1992): Isolation and structure of a brain constituent that binds to the cannabinoid receptor. Science $\underline{258}, 1946-9$ 
Dietz L, Glaz-Sandberg A, Nguyen H, Skopp G, Mikus G, Aderjan R (2007): The urinary disposition of intravenously administered 11-nor-9-carboxy-delta-9tetrahydrocannabinol in humans. Ther Drug Monit 29, 368-72

Ding X, Kaminsky LS (2003): Human extrahepatic cytochromes P450: function in xenobiotic metabolism and tissue-selective chemical toxicity in the respiratory and gastrointestinal tracts. Annu Rev Pharmacol Toxicol 43, 149-73

Drummer OH, Gerostamoulos J, Batziris H, Chu M, Caplehorn J, Robertson MD, Swann P (2004): The involvement of drugs in drivers of motor vehicles killed in Australian road traffic crashes. Accid Anal Prev $\underline{36}$, 239-48

Evans WE, McLeod HL (2003): Pharmacogenomics - drug disposition, drug targets and side effects. N Engl J Med $\underline{348}, 538-49$

Gérard CM, Mollereau C, Vassart G, Parmentier M (1991): Molecular cloning of a human cannabinoid receptor which is also expressed in testis. Biochem $\mathrm{J} \underline{279}, 129$ 34

Girennavar B, Jayaprakasha GK, Patil BS (2007): Potent inhibition of human cytochrome P450 3A4, 2D6, and 2C9 isoenzymes by grapefruit juice and its furocoumarins. J Food Sci $\underline{72}$, 417-21

Giroud C, Augsburger M, Favrat B, Menetrey A, Pin MA, Rothuizen LE, Appenzeller M, Buclin T, Mathieu S, Castella V, Hazekamp A, Mangin P(2006): Effects of oral cannabis and dronabinol on driving capacity. Ann Pharm Fr $\underline{64}$, 161-72

Glaz-Sandberg A, Dietz L, Nguyen H, Oberwittler H, Aderjan R, Mikus G (2007): Pharmacokinetics of 11-nor-9-carboxy-Delta(9)-tetrahydrocannabinol (CTHC) after intravenous administration of CTHC in healthy human subjects. Clin Pharmacol Ther $\underline{82}, 63-9$

Goldstein JA (2001): Clinical relevance of genetic polymorphisms in the human CYP2C subfamily. Br J Clin Pharmacol 52, 349-355 
Goldstein JA, de Morais SM (1994): Biochemistry and molecular biology of human CYP2C subfamily. Pharmacogenetics $\underline{4}, 285-299$

Goodwin RS, Gustafson RA, Barnes A, Nebro W, Moolchan ET, Huestis MA (2006): Delta(9)-tetrahydrocannabinol, 11-hydroxy-delta(9)-tetrahydrocannabinol and 11-nor9-carboxy-delta(9)-tetrahydrocannabinol in human plasma after controlled oral administration of cannabinoids. Ther Drug Monit $\underline{28}, 545-51$

Grotenhermen F (1999): [The effects of cannabis and THC]. Forsch Komplementarmed $\underline{3}, 7-11$

Grotenhermen F (2003): Pharmacokinetics and pharmacodynamics of cannabinoids. Clin Pharmacokinet $\underline{42}$, 327-60

Grotenhermen F (2004): Pharmacology of cannabinoids. Neuro Endocrinol Lett $\underline{25}$, 14-23

Guo LQ, Chen QY, Wang X, Liu YX, Chu XM, Cao XM, Li JH, Yamazoe Y (2007): Different roles of pummelo furanocoumarin and cytochrome P450 $3 A 5^{*} 3$ polymorphism in the fate and action of felodipine. Curr Drug Metab $\underline{8}, 623-30$

Gustafson RA, Kim I, Stout PR, Klette KL, George MP, Moolchan ET, Levine B, Huestis MA (2004): Urinary pharmacokinetics of 11-nor-9-carboxy-delta9tetrahydrocannabinol after controlled oral delta9-tetrahydrocannabinol administration. J Anal Toxicol 28, $160-7$

Hagenbach U, Luz S, Ghafoor N, Berger JM, Grotenhermen F, Brenneisen R, Mäder M (2007): The treatment of spasticity with Delta-9-tetrahydrocannabinol in persons with spinal cord injury. Spinal Cord $\underline{45}, 551-62$

Hagenbuch B, Meier PJ (2003): The superfamily of organic anion transporting polypeptides. Biochim Biophys Acta 1609, 1-18 
Hagenbuch B, Meier PJ (2004): Organic anion transporting polypeptides of the OATP/SIc21 family: phylogenetic classification as OATP/SLCO superfamily, new nomenclature and molecular/functional properities. Pflugers Arch 447, 653-65

Hall W, Christie M, Currow D (2005): Cannabinoids and Cancer: causation, remediation and palliation. Lancet Oncol $\underline{6}, 35-42$

Herscovitch J, Broughton R (1981): Sensitivity of the stanford sleepiness scale to the effects of cumulative partial sleep deprivation and recovery oversleeping. Sleep $\underline{4}$, 83-91

Hohmann AG, Suplita RL, Bolton MN, Neely MH, Fegley D, Mangieri R, Krey JF, Walker JM, Holmes PV, Crystal JD, Duranti A, Tontini A, Mor M, Tarzia G, Piomelli D (2005): An endocannabinoid mechanism for stress-induced analgesia. Nature $\underline{435}$, 1108-12

Hopfer CJ, Young SE, Purcell S, Crowley TJ, Stallings MC, Corley RP, Rhee SH, Smolen A, Krauter K, Hewitt JK, Ehringer MA (2006): Cannabis receptor haplotype associated with fewer cannabis dependence symptoms in adolescents. Am J Med Genet B Neuropsychiatr Genet 141, 895-901

Huestis MA, Mitchell JM, Cone EJ (1995): Detection times of marijuana metabolites in urine by immunoassay and GC-MS. J Anal Toxicol $\underline{19}$, 443-9

Huestis MA, Barnes A, Smith ML (2005): Estimating the time of last cannabis use from plasma delta9-tetrahydrocannabinol concentrations. Clin Chem $\underline{51}$, 2289-95

Huestis MA, Elsohly M, Nebro W, Barnes A, Gustafson RA, Smith ML (2006): estimating time of last oral ingestion of cannabis from plasma $\mathrm{THC}$ and $\mathrm{THCCOOH}$ concentrations. Ther Drug Monit $\underline{28}, 540-4$ 
Hustert E, Haberl M, Burk O, Wolbold R, He YQ, Klein K, Nuessler AC, Neuhaus P, Klattig J, Eiselt R, Koch I, Zibat A, Brockmöller J, Halpert JR, Zanger UM, Wojnowski L (2001): The genetic determinants of the CYP3A5 polymorphism. Pharmacogenetics $\underline{11}, 773-9$

Iwasaki K (2007): Metabolism of tacrolimus (FK506) and recent topics in pharmacokinetics. Drug Metab Pharmacokinet 22, 328-35

Izzo AA, Coutts AA (2005): Cannabinoids and the digestive tract. Handb Exp Pharmacol $\underline{168}, 573-98$

Janke W, Debus G: Die Eigenschaftswörterliste (EWL). Eine mehrdimensionale Methode zur Beschreibung von Aspekten des Befindens. Hogrefe Verlag, Göttingen 1978

Jin Y, Wang YH, Miao J, Li L, Kovacs RJ, Marunde R, Hamman MA, Phillips S, Hilligoss J, Hall SD (2007): Cytochrome P450 3A5 genotype is associated with verapamil response in healthy subjects. Clin Pharmacol Ther $\underline{82}, 579-85$

Johnson JR, Jennison TA, Peat MA, Foltz RL (1984): Stability of delta 9tetrahydrocannabinol (THC), 11-hydroxy-THC, and 11-nor-9-carboxy-THC in blood and plasma. J Anal Toxicol $\underline{8}, 202-4$

Khiabani HZ, Bramness JG, Bjørneboe A, Mørland J (2006): Relationship between THC concentration in blood and impairment in apprehended drivers. Traffic Inj Prev $\underline{7}, 111-6$

Kirchheiner J, Brockmöller J (2005): Clinical consequences of cytochrome P450 2C9 polymorphismus. Clin Pharmacol Ther $\underline{77}, 1-16$

Kirchheiner J, Tsahuridu M, Jabrane W, Roots I, Brockmöller J (2004): The CYP2C9 polymorphism: from enzyme kinetics to clinical dose recommendations. Personalized Med 1, 1-22 
Kuehl P, Zhang J, Lin Y, Lamba J, Assem M, Schuetz J, Watkins PB, Daly A, Wrighton SA, Hall SD, Maurel P, Relling M, Brimer C, Yasuda K, Venkataramanan R, Strom S, Thummel K, Boguski MS, Schuetz E (2001): Sequence diversity in CYP3A promoters and characterization of the genetic basis of polymorphic CYP3A5 expression. Nat Genet 27, 383-91

Lazarou J, Pomeranz BH, Corey PN (1998): Incidence of adverse drug reactions in hospitalized patients: a meta-analysis of prospective studies. JAMA $\underline{279}, 1200-5$

Lee CR, Goldstein JA, Pieper JA (2002): Cytochrome P450 2C9 polymorphism: a comprehensive review of the in-vitro and human data. Pharmacogenetics $\underline{12}, 251-63$

Lemberger L, Silberstein SD, Axelrod J, Kopin IJ (1970): Marihuana: studies on the disposition and metabolism of delta-9-tetrahydrocannabinol in man. Science $\underline{170}$, 1320-2

Ling G, Gu J, Genter MB, Zhuo X, Ding X (2004): Regulation of cytochrome P450 gene expression in the olfactory mucosa. Chem Biol Interact $\underline{147}, 247-58$

Lötsch J, Lange C, Hummel T (2004): A simple and reliable method for clinical assessment of odor thresholds. Chem Senses $\underline{29}, 311-7$

Loh PT, Lou HX, Zhao Y, Chin YM, Vathsala A (2008): Significant impact of gene polymorphisms on tacrolimus but not cyclosporine dosing in asian renal transplant recipients. Transplant Proc $\underline{40}, 1690-5$

Lovibond PF, Lovibond SH (1995): The structure of negative emotional states: comparison of the Depression Anxiety Stress Scale (DASS) with the Beck Depression and Anxiety Inventories. Behav Res Ther $\underline{33}$, 335-43

Lu D, Vemuri VK, Duclos RI Jr, Makriyannis A (2006): The cannabinergic system as a target for anti-inflammatory therapies. Curr Top Med Chem $\underline{6}, 1401-26$ 
McCabe M, Smith FP, Macdonald JS, Woolley PV, Goldberg D, Schein PS (1988): Efficiency of tetrahydrocannabinol in patients refractory to standard antiemetic therapy. Invest New Drugs $\underline{6}, 243-6$

Manzanares J, Julian M, Carrascosa A (2006): Role of cannabinoid system in pain control and therapeutic implications for the management of acute and chronic pain episodes. Curr Neuropharmacol $\underline{4}, 239-57$

Maralikova B, Weinmann W (2004): Simultaneous determination of Delta9tetrahydrocannabinol, 11-hydroxy-Delta9-tetrahydrocannabinol and 11-nor-9carboxy-Delta9-tetrahydrocannabinol in human plasma by high-performance liquid chromatography/tandem mass spectrometry. J Mass Spectrom $\underline{39}, 526-31$

McGilveray IJ (2005): Pharmakokinetics of cannabinoids. Pain Res Manag $\underline{10}$, 15A$22 \mathrm{~A}$

Meisel C, Gerloff T, Kirchheiner J, Mrozikiewicz PM, Niewinski P, Brockmöller J, Roots I (2003): Implications of pharmacogenetics for individualizing drug treatment and for study design. J Mol Med $\underline{81}, 154-67$

Melchior M, Chastang JF, Goldberg P, Fombonne E (2008): High prevalence rates of tobacco, alcohol and drug use in adolescents and young adults in France: results from the GAZEL Youth study. Addict Behav $\underline{33}$, 122-33

Miners JO, Birkett DJ (1998): Cytochrome P4502C9: an enzyme of major importance in human drug metabolism. Br J Clin Pharmacol 45, 525-38

Moore TH, Zammit S, Lingford-Hughes A, Barnes TR, Jones PB, Burke M, Lewis G (2007): Cannabis use and risk of psychotic or affective mental health outcomes: a systematic review. Lancet $\underline{370}, 319-28$

Musshoff F, Madea B (2006): Review of biologic matrices (urine, blood, hair) as indicators of recent or ongoing cannabis use. Ther Drug Monit $\underline{28}, 155-63$ 
Nadulski T, Pragst F, Weinberg G, Roser P, Schnelle M, Fronk EM, Stadelmann AM (2005): Randomized, Double-Blind, Placebo-Controlled Study About the Effects of Cannabidiol (CBD) on the Pharmacokinetics of Delta9-Tetrahydrocannabinol (THC) After Oral Application of THC Verses Standardized Cannabis Extract. Ther Drug Monit 27, 799-810

Ohlsson A, Lindgreen JE, Wahlen A, Agurell S, Hollister LE, Gillespie HK (1980): Plasma delta- 9 tetrahydrocannabinol concentrations and clinical effects after oral and intravenous administration and smoking. Clin Pharmacol Ther $\underline{28}, 409-16$

Ohyama K, Nakajima M, Suzuki M, Shimada N, Yamazaki H, Yokoi T (2000): Inhibitory effects of amiodarone and its $\mathrm{N}$-deethylated metabolite on human cytochrome P450 activities. Br J Clin Pharmacol $\underline{49}$, 244-53

Peeters A, Beckers S, Mertens I, Van Hul W, Van Gaal L (2007): The G1422A variant of the cannabinoid receptor gene (CNR1) is associated with abdominal adiposity in obese men. Endocrine 31, 138-41

Ramaekers JG, Moeller MR, van Ruitenbeek P, Theunissen EL, Schneider E, Kauert G (2006): Cognition and motor control as a function of Delta9-THC concentration in serum and oral fluid: limits of impairment. Drug Alcohol Depend $\underline{85}, 114-22$

Rettie AE, Wienkers LC, Gonzales FJ, Trager WF, Korzewka KR (1994): Impaired (S)-warfarin metabolism catalysed by the R144C allelic variant of CYP2C9. Pharmacogenetics $\underline{4}, 39-42$

Ring A, Tothfalusi L, Endrenyi L, Weiss M (2000): Sensitivity of empirical metrics of rate of absorption in bioequivalence studies. Pharm Res $\underline{17}, 583-8$.

Russo P, Strazzullo P, Cappuccio FP, Tregouet DA, Lauria F, Loguerico M, Barba G, Versiero M, Siani A (2007): Genetic variations at the endocannabinoid type 1receptor gene (CNR1) are associated with obesity phenotypes in men. J Clin Endocrinol Metab $\underline{92}, 2382-6$ 
Scheen AJ (2007): Drug-drug and food-drug pharmacokinetic interactions with new insulinotropic agents repaglinide and nateglinide. Clin Pharmacokinet $\underline{46}, 93-108$

Schmidt LG, Samochowiec J, Finckh U, Fiszer-Piosik E, Horodnicki J, Wendel B, Rommelspacher H, Hoehe MR (2002): Association of a CB1 cannabinoid receptor gene (CNR1) polymorphism with severe alcohol dependence. Drug Alcohol Depend $\underline{65}, 221-4$

Schuetz JD, Molowa DT, Guzelian PS (1989): Characterization of a cDNA encoding a new member of the glucocorticoid-responsive cytochromes P450 in human liver. Arch Biochem Biophys 274, 355-65

Shimada T, Yamazaki H, Mimura M, Inui Y, Guengerich FP (1994): Interindividual variations in human liver cytochrome P-450 enzymes involved in the oxidation of drugs, carcinogens and toxic chemicals: studies with liver microsomes of 30 Japanese and 30 Caucasians. J Pharmacol Exp Ther 270, 414-23

Skopp G, Pötsch L (2002): Stability of 11-nor-delta(9)-carboxy-tetrahydrocannabinol glucuronide in plasma and urine assessed by liquid chromatography-tandem mass spectrometry. Clin Chem $\underline{48}$, 301-6

Smith RA Jr 1971: Studies of temporal frequency adaptation in visual contrast sensitivity. J Physiol 216, 531-52

Sullivan-Klose TH, Ghanayem BI, Bell DA, Zhang ZY, Kaminsky LS, Shenfield GM, Miners JO, Birkett DJ, Goldstein JA (1996): The role of CYC2C9-Leu359 allelic variant in tolbutamide polymorphism. Pharmacogenetics $\underline{6}, 341-349$

Thompson GR, Rosenkrantz H, Schaeppi UH, Braude MC (1973): Comparison of acute oral toxicity of cannabinoids in rats, dogs and monkeys. Toxicol Appl Pharmacol 25, 363-72.

THC Pharm GmbH: THC Health Concept, THC Pharm GmbH, Frankfurt/Main, o. J. 
Timpone JG, Wright DJ, Li N, Egorin MJ, Enama ME, Mayers J, Galetto G (1997): The safety and pharmacokinetics of single-agent and combination therapy with megestrol acetate and dronabinol for the treatment of HIV wasting syndrome. The DATRI 004 Study Group. Division of AIDS Treatment Research Initiative. AIDS Res Hum Retroviruses $\underline{13}$, 305-15

Tomalik-Scharte D, Lazar A, Fuhr U, Kirchheiner J (2008): The clinical role of genetic polymorphisms in drug-metabolizing enzymes. Pharmacogenomics $\underline{8}, 4-15$

Tossmann P (2006): The use of cannabis in the Federal Republic of Germany. Prax Kinderpsychol Kinderpsychiatr $\underline{55}$, 509-19

Ujike H, Takaki M, Nakata K, Tanaka Y, Takeda T, Kodama M, Fujiwara Y, Sakai A, Kuroda S (2002): CNR1, central cannabinoid receptor gene, associated with susceptibility to hebephrenic schizophrenia. Mol Psychiatry $\underline{7}, 515-8$

Vormfelde SV, Schirmer M, Tolia MR, Meineke I, Kirchheiner J, Nürnberg P, Brockmöller J (2007): Genetic variation at the CYP2C locus and its association with torsemide biotransformation. Pharmacogenomics J $\underline{7}, 200-11$

Vormfelde SV, Tolia MR, Schirmer M, Meineke I, Nürnberg P, Brockmöller J (2008): The polymorphisms Asn130Asp and Val174Ala in OATP1B1 and the CYP2C9 allele *3 independently affect torsemide pharmacokinetics and pharmacodynamics. Clin Pharmacol Ther $\underline{83}, 815-7$

Wall ME, Perez-Reyes M (1981): The metabolism of delta 9-tetrahydrocannabinol and related cannabinoids in man. J Clin Pharmacol 21, 178-89

Wall ME, Sadler BM, Brine D, Taylor H, Perez-Reyes M (1983): Metabolism, disposition, and kinetics of delta-9-tetrahydrocannabinol in men and women. Clin Pharmacol Ther $\underline{34}, 352-63$ 
Watanabe K, Matsunaga T, Kimura T, Funahashi T, Funae Y, Ohshima T, Yamamoto I (2002): Major cytochrome P450 enzymes responsible for microsomal aldehyde oxygenation of 11-oxo-Delta-8-tetrahydrocannabinol and 9-anthraldehyde in human liver. Drug Metab Pharmacokinet $\underline{17}, 516-21$

Watanabe K, Yamaori S, Funahashi T, Kimura T, Yamamoto I (2007): Cytochrome P450 enzymes involved in the metabolism of tetrahydrocannabinols and cannabinol by human hepatic microsomes. Life Sci $\underline{80}, 1415-9$

Williams PA, Cosme J, Ward A, Angove HC, Matak Vinković D, Jhoti H (2003): Crystal structure of human cytochrome P450 2C9 with bound warfarin. Nature $\underline{424}$, 464-8

Williamson EM, Evans FJ (2000): Cannabinoids in clinical practise. Drugs $\underline{60}$, 130314

Zajicek J, Fox P, Sanders H, Wright D, Vickery J, Nunn A, Thompson A; UK MS Research Group (2003): Cannabinoids for treatment of spasticity and other symptoms related to multiple sclerosis (CAMS study): multicentre randomised placebo-controlled trial. Lancet $\underline{362}, 1517-26$

Zigmond AS, Snaith RP (1983): The hospital anxiety and depression scale. Acta Psychiatr Scand $\underline{67}, 361-70$

Zuo L, Kranzler HR, Luo X, Covault J, Gelernter J (2007): CNR1 variation modulates risk for drug and alcohol dependence. Biol Psychiatry $\underline{62}, 616-26$ 


\section{Danksagung}

Mein besonderer Dank gilt Herrn Prof. Dr. Jürgen Brockmöller für die freundliche Überlassung des Dissertationsthemas und insbesondere für die kontinuierliche und umfassende Betreuung bei der Niederschrift.

Ich danke Herrn Daniel Sehrt und Frau Dr. Cordula Sachse-Seeboth für die Planung der Studiendurchführung und die Hilfestellung während des praktischen Abschnitts der Studie.

Mein weiterer Dank gilt allen Labormitarbeitern und -mitarbeiterinnen der Abteilung der Klinischen Pharmakologie Göttingen, insbesondere Frau Ellen Bruns und Frau Monika Winkler.

Für das Korrekturlesen dieser Arbeit möchte ich meinem Bruder, Christian Pfeil und meinen Freunden Karsten Schuldt, Christian Klein und Lena Conradi herzlichst danken.

Herrn Tobias Gohla danke ich für seine allumfassende Hilfestellung bei allen Fragen der Textverarbeitung. 


\section{Lebenslauf}

Ich, Johannes Pfeil, wurde am 07.02.1983 als zweites Kind meiner Eltern Ulrike und Walter Pfeil, in Rostock geboren. Mein Bruder, Christian Pfeil, kam am 27.12.1978 zur Welt.

Nachdem ich von 1989 bis 1993 zunächst die Grundschule in Rostock besuchte, wechselte ich im September 1993 auf das Johann-Heinrich-von-Thünen-Gymnasium in Rostock, wo ich meine Schulausbildung im August 2002 mit dem Abitur beendete. Im Anschluss daran absolvierte ich von Oktober 2002 bis Juni 2003 meinen Grundwehrdienst. Dabei erfolgte zunächst die dreimonatige allgemeine Grund- und Sanitätsausbildung in Feldkirchen/Niederbayern. Die folgenden sechs Monate war ich auf dem Flughafen Rostock/Laage als Assistenz des Fliegerarztes tätig. Im Wintersemester 2003/04 begann ich mein Medizinstudium an der Georg-AugustUniversität Göttingen, wo ich im April 2006 den Ersten Abschnitt der Ärztlichen Prüfung erfolgreich absolvierte. Derzeit verbringe ich den klinischen Studienabschnitt ebenfalls in Göttingen. Im August 2009 werde ich das Praktische Jahr beginnen.

Seit Oktober 2006 übe ich eine studentische Tätigkeit im Aufwachraum des Krankenhauses Neu Betlehem in Göttingen aus. 